medRxiv preprint doi: https://doi.org/10.1101/2022.02.17.22271136; this version posted February 18, 2022. The copyright holder for this preprint (which was not certified by peer review) is the author/funder, who has granted medRxiv a license to display the preprint in It is made available under a CC-BY-NC-ND 4.0 International license .

February 16th, 2021

\title{
Immune system and blood-brain barrier-wide biomarker analyses provide causal evidence for autoimmunity in dementia
}

Joni V. Lindbohm, MD, PhD $;{ }^{1,2}$ Nina Mars, MD, PhD $;{ }^{3}$ Pyry N. Sipilä, MD, PhD $;{ }^{2}$ Prof. Archana Singh-Manoux; ${ }^{1,4}$ Heiko Runz, MD, PhD; ${ }^{5,6}$ FinnGen; ${ }^{3}$ Prof. Gill Livingston; ${ }^{7,8}$ Prof. Sudha Seshadri; $;, 10,11,12$ Prof. Ramnik Xavier; ${ }^{13,14}$ Prof. Aroon D. Hingorani; ${ }^{15,16,17}$ Prof. Samuli Ripatti; ${ }^{2,3,18 *}$ Prof. Mika Kivimäki ${ }^{1,2 *}$

${ }^{1}$ Department of Epidemiology and Public Health, University College London, 1-19 Torrington Place, London, UK

${ }^{2}$ Clinicum, Department of Public Health, University of Helsinki, P.O. Box 41, FI-00014 Helsinki, Finland

${ }^{3}$ Institute for Molecular Medicine Finland (FIMM), HiLIFE, University of Helsinki, Helsinki, Finland

${ }^{4}$ Université de Paris, Inserm U1153, Epidemiology of Ageing and Neurodegenerative diseases, Paris, France

${ }^{5}$ Molecular Medicine Partnership Unit (MMPU), University of Heidelberg/EMBL, Heidelberg, Germany

${ }^{6}$ Translational Biology, Biogen Inc, Cambridge, MA, USA

${ }^{7}$ Division of Psychiatry, University College London, London, UK

${ }^{8}$ Camden and Islington NHS Foundation Trust, London, UK

${ }^{9}$ Glenn Biggs Institute of Alzheimer's and Neurodegenerative Diseases, University of Texas Health Science Center, San Antonio, TX, USA.

${ }^{10}$ Boston University School of Public Health, Boston, MA, USA.

${ }^{11}$ New York University Grossman School of Medicine, New York, NY, USA.

12 Boston University School of Medicine, Boston, MA, USA.

${ }^{13}$ Center for Computational and Integrative Biology, Massachusetts General Hospital and Harvard Medical School, Boston, MA, USA

${ }^{14}$ Department of Molecular Biology, Massachusetts General Hospital and Harvard Medical School, Boston, MA, USA

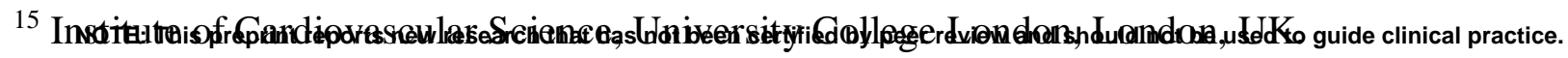


medRxiv preprint doi: https://doi.org/10.1101/2022.02.17.22271136; this version posted February 18, 2022. The copyright holder for this preprint (which was not certified by peer review) is the author/funder, who has granted medRxiv a license to display the preprint in It is made available under a CC-BY-NC-ND 4.0 International license .

2

${ }^{16}$ University College London, British Heart Foundation Research Accelerator, London, UK.

${ }^{17}$ Health Data Research UK, London, UK.

${ }^{18}$ Broad Institute of the Massachusetts Institute of Technology and Harvard University, Cambridge, MA, USA

* Authors contributed equally

Correspondence to Dr Joni Lindbohm, Clinicum, Department of Public Health, University of Helsinki, P.O. Box 41, FI-00014 Helsinki, Finland; E-mail address: joni.lindbohm@ helsinki.fi; Telephone: +358 91911 Fax: +358919127600 
medRxiv preprint doi: $h$ ttps://doi.org/10.1101/2022.02.17.22271136; this version posted February 18, 2022. The copyright holder for this preprint (which was not certified by peer review) is the author/funder, who has granted medRxiv a license to display the preprint in It is made available under a CC-BY-NC-ND 4.0 International license .

3

\begin{abstract}
Immune system and blood brain barrier (BBB) dysfunction are implicated in the development of Alzheimer's disease and other dementias, but their causal role remains unknown. We performed Mendelian randomization (MR) for over 43,643 immune system and BBB-related biomarkers and identified 126 potential causal risk factors for dementias. A phenome-wide analysis using MRbased polygenic risk score in FinnGen study $(\mathrm{N}=339,233)$ for these risk factors revealed a common genetic background for dementias and autoimmune diseases which was supported by further HLA analyses. Pathway analyses linked the 126 proteins to amyloid- $\beta$, tau and $\alpha$-synuclein pathways, increased inflammatory responses, and altered self-tolerance mechanisms. In inverse-probabilityweighted analyses simulating randomized controlled drug trials in observational data, antiinflammatory and immunosuppressive medications were associated with reduced dementia risk ( $\mathrm{p}<0.01$ for methotrexate and TNF- $\alpha$ inhibitors). These converging results from different research lines suggest that autoimmunity is a modifiable component in diseases causing dementia.
\end{abstract}


medRxiv preprint doi: https://doi.org/10.1101/2022.02.17.22271136; this version posted February 18, 2022. The copyright holder for this preprint (which was not certified by peer review) is the author/funder, who has granted medRxiv a license to display the preprint in

It is made available under a CC-BY-NC-ND 4.0 International license.

\section{Background}

3 Dysregulation of peripheral and central immune systems and chronic neuroinflammation occur in diseases, such as severe infections and autoimmune diseases that relate to increased risk of dementia. ${ }^{1-3}$ In central immune system, neuroinflammation can closely relate to sustained activation of microglia, the resident immune cells in the central nervous system (CNS), and lead to neurodegeneration and increased deposition of plaques and tangles. ${ }^{1-3}$ When CNS encounters infections, trauma, or toxins, microglia are key in the acute immune response and in this phase are neuroprotective. When the microglia activation continues, this leads to chronic production of proinflammatory products including reactive oxygen species, nitric oxide, and cytokines. ${ }^{1-3}$ This in turn may exacerbate disease-specific pathology, such as amyloid $\beta$ and tau protein in Alzheimer's disease, and neurodegeneration. ${ }^{1}$

In addition, the peripheral immune system and blood brain barrier are thought to be important in the development of dementias. ${ }^{1,4}$ Because of the crosstalk between the central and peripheral immune system and BBB, the causal factors linking inflammation to dementias may originate from any of these systems. Chronic peripheral inflammation, from low-grade systemic inflammation ${ }^{5,6}$ and infections, ${ }^{7}$ can disrupt BBB so that it allows neurotoxic and pro-inflammatory plasma components and pathogens to enter CNS. ${ }^{4}$ Dysfunctional BBB may also promote peripherally driven neuroinflammation by increasing endothelial adhesion molecules and chemokines and migration of peripheral leukocytes to CNS. ${ }^{4}$ These processes expose CNS to prolonged neuroinflammation and subsequent neurodegeneration, ${ }^{1}$ although the role of centrally and peripherally driven CNS autoimmunity in dementias remains unclear..$^{8-11}$ In addition, there is limited data to confirm the causal nature of these hypothesised mechanisms. ${ }^{1-3}$

Mendelian randomization (MR), using large GWAS libraries for unconfounded genetic proxies for biomarkers, and triangulation, combining multiple research methods with different sources of strengths and biases to identify convergent evidence, improve validity of causal estimates and success rate in drug development. ${ }^{12,13}$ Although a range of studies on the immune system and BBB have been conducted, ${ }^{1-4}$ to the best of our knowledge, there are currently no published large-scale, immune system and BBB-wide MR analyses, confirmed by triangulation to identify novel drug targets and risk factors for dementias.

In this study, we analyse biomarkers of immune system and BBB (Figure 1) and use MR to study potential causal associations with five outcomes (Alzheimer's disease, Parkinson's diseases, frontotemporal dementia, vascular dementia, and poor cognitive performance) and triangulate the results. We identify several novel associations between inflammatory and immune checkpointrelated biomarkers and dementias. Using single nucleotide polymorphisms (SNPs) strongly associated with these biomarkers, we create MR-based polygenic risk scores (PRS) for the diseases. In phenome-wide association analyses (PheWAS) for the PRSs, we observe shared genetic risk factors with autoimmune diseases and dementias, which are supported by HLA analysis. In line with this, our pathway analyses for risk factors identified in MR show enrichment in inflammatory and self-tolerance related processes, which are in close interaction with amyloid $\beta$, tau protein, and $\alpha$-synuclein. Lastly, using inverse-probability-weighted (IPW) survival analysis to simulate a 
medRxiv preprint doi: $h t$ tps://doi.org/10.1101/2022.02.17.22271136; this version posted February 18, 2022. The copyright holder for this preprint (which was not certified by peer review) is the author/funder, who has granted medRxiv a license to display the preprint in

It is made available under a CC-BY-NC-ND 4.0 International license .

randomized controlled trial design in observational data with up to 20 years of follow-up, we identify anti-inflammatory medications which may lower the risk of dementias. In summary, we detect several novel risk factors and potential drug targets for dementia-causing diseases in a triangulation framework and propose that dementias may have an autoimmune component which is modifiable with anti-inflammatory medication. These findings are set out in Figure 2.

\section{Results}

\section{Mendelian randomization and proteomics analyses}

In the discovery step, we used MR to identify potential causal risk factors for dementias. The MRbase search provided 30,913 components for the immune system and 12,730 components for BBB; 254 immune system components and 132 BBB components passed the false discovery rate (FDR) of 5\% (Figure 1). After removal of duplicates, 126 unique biomarkers were associated (p-value $<0.000502$ ) with neurodegenerative diseases in MR analyses using Wald ratio (when only 1 or 2 SNPs was available) or inverse variance-weighted method (when 3 or more SNPs were available) (Figure 3A and B). MR sensitivity analyses showed no strong evidence of reverse causality, and evidence of horizontal pleiotropy for only two outcomes. (STable 1 and SFigures 1 A and B). The 126 biomarkers (FDR <5\%) showed few off-target associations, mainly with type 1 diabetes and LDL-C. (SFigure 2).

While most biomarkers were disease-specific, 8 were associated with Alzheimer's disease, vascular dementia, and poor cognitive performance. The SNPs for the latter biomarkers were centred within 500 kilobases $(\mathrm{kb})$ from $A P O E$ gene, one of the strongest genetic risk factors for late-onset Alzheimer's disease (SFile 1). To differentiate the effects of these biomarkers from APOE, we studied dementia outcomes and plasma proteins measured within the Whitehall II cohort $(\mathrm{N}=6,235)$. Out of the 8 proteins, 2 were associated with all-cause dementia in a 20-year follow-up and 1 (IFIT2) of these remained after adjustment of $A P O E$ status (SFigure 3; data on dementia subtypes were not available).

The 118 outcome-specific biomarkers did not show similar enrichment around high-risk genes for Alzheimer's disease such as APP, PSEN1, PSEN2, ADAM10, TREM2, PLD3, and UNC5C. Instead, they were characterized by inflammatory, chemokine, complement, and adhesion processes $(\mathrm{C} 1 \mathrm{Q}$, C1R, C4B, CCL1, CDHR5, GPNMB, IL1 $\beta$, IL17, IL27, IL37, LTBR, PTP1B, SIGIRR), antigen presenting and immune checkpoints (HLA-DR, HLA-DQ, BAFFR, C1R, C1Q, CD11, CD19, CD20, CD33, CD40, CX3CR1, PD-1, and PDL-1), and BBB tight junction related biomarkers (TJP, AIMP1, BIN1).

\section{Polygenic risk score and HLA analysis}

To study the diseases that share common genetic background with dementias, we created MR-based polygenetic risk score (PRS) using the SNPs of the 126 biomarkers for dementias and then run a phenome-wide association analysis (PheWAS) (Figure 4). After linkage disequilibrium (LD) pruning and SNP matching in FinnGen $(\mathrm{N}=339,233), 92$ SNPs were available for a PRS for 
medRxiv preprint doi: https://doi.org/10.1101/2022.02.17.22271136; this version posted February 18,2022 . The copyright holder for this preprint (which was not certified by peer review) is the author/funder, who has granted medRxiv a license to display the preprint in

It is made available under a CC-BY-NC-ND 4.0 International license .

Alzheimer's disease (the number of SNPs for other outcomes was insufficient, $\leq 25$ ). The PRS was strongly and positively associated with risk of all types of dementia-causing diseases (except Parkinson's disease) and autoimmune disorders, such as rheumatic diseases, type 1 diabetes and its complications. The PRS was also associated with increased risk of thyroiditis, coronary heart disease, hyperlipidaemia, and the likelihood of having medications for Crohn's disease (indicating disease severity), but reduced risk of cancers. We studied whether HLA-types that predispose to autoimmune diseases are also associated with dementias. These analyses showed 21 directionally consistent HLA association with dementias and at least one autoimmune disease (Figure 5A-E).

\section{Pathway analyses}

To identify pathways the 126 biomarkers are involved in, we performed analyses based on KEGG, ClueGO, and ConsensusPathDB databases. The findings provided further support for the autoimmune component in dementias. KEGG and ClueGO analyses showed that the identified biomarkers increased MHC-II-mediated antigen presentation in several processes, HLA-DR expression across all hematopoietic cell lines, neuronal adhesion molecule CNTN2, and PD-L1 in T-cell-antigen interactions that reduces self-tolerance. The biomarkers also decreased barrier protecting IL-17F, self-tolerance increasing PDCD1 in T-cell-antigen interactions, and antiviral complement factor B and IFNAR1 (SFigures 4A-H and 5). ConsensusPathDB analyses showed that all of the 42 plasma proteins included in the 126 biomarkers had physical interactions with or were in a biological pathway within zero to two molecule distance from amyloid precursor protein, tau protein, or $\alpha$-synuclein (SFigure 6A-R).

\section{Inverse probability-weighted analyses}

To evaluate the autoimmune hypothesis in relation to modifiability and drug repurposing, we used inverse probability-weighted (IPW) analyses in FinnGen and studied whether commonly used antiinflammatory and immunosuppressive medications are likely to reduce the risk of dementias (Table 1). We validated the IPW protocol with a positive control, by replicating the randomized controlled trial (RCT) effect between statin medication and myocardial infarction and used anti-inflammatory medications as negative control. ${ }^{14-17}$ Supporting the autoimmune hypothesis, risk of dementiacausing diseases was reduced with methotrexate and TNF- $\alpha$ inhibitors. Methotrexate appeared to decrease risk only in those with above-average MR-based autoimmune PRS (50\% cut-off). To identify rare medications not detectable in FinnGen with sufficient power, we additionally searched the OpenTargets database. This search identified 64 drugs that target 18 of the 126 proteins, suggesting that these may have potential for repurposing to treatment of dementias (SFile 2).

\section{Discussion}

Using triangulation across Mendelian randomization, plasma proteomics, polygenic risk scores, HLA type, pathway, and IPW analyses, we identified causal support for 126 risk factors and potential drug targets for dementia-causing diseases. Of these 118 were novel MR associations. Most of the identified risk factors and targets were outcome specific, and included inflammatory, self-tolerance, and/or BBB tight junction related biomarkers. A phenome-wide analysis of PRS from SNPs associated with the identified risk factors showed that Alzheimer's disease share genetic background with autoimmune diseases, such as rheumatoid arthritis and type 1 diabetes. The 
medRxiv preprint doi: https://doi.org/10.1101/2022.02.17.22271136; this version posted February 18, 2022. The copyright holder for this preprint (which was not certified by peer review) is the author/funder, who has granted medRxiv a license to display the preprint in

It is made available under a CC-BY-NC-ND 4.0 International license.

autoimmune hypothesis was also supported by HLA analyses showing 21 HLA-type associations common to dementias and autoimmune diseases. Pathway analyses linked the 126 biomarkers to autoimmunity via antigen presentation and reduced self-tolerance and showed that all 42 plasma proteins of the 126 biomarkers are closely related to $\alpha$-synuclein, amyloid precursor, and tau protein pathways that characterize Alzheimer's and Parkinson's diseases and dementia with Levy bodies. The autoimmune hypothesis was further supported by IPW analyses mimicking randomized controlled drug trial in observational data. They suggested anti-inflammatory medication use may potentially reduce the risk of these illnesses.

Several lines of earlier and current evidence support the hypothesis that diseases causing dementia have an autoimmune component (Figure 2). Epidemiological studies have shown higher rates of cognitive impairment, Alzheimer's diseases, Parkinson's disease, vascular, and frontotemporal dementia in rheumatic disease patients. ${ }^{18-22}$ Diabetes is a recognized risk factor for Alzheimer's disease $^{23}$ and individuals with type 1 diabetes have higher rate of cognitive decline, Alzheimer's disease, and vascular dementia. ${ }^{24,25}$ Thyroiditis, which often has an autoimmune origin, increases risk of dementias. ${ }^{10,26}$ In agreement with these findings, we showed that MR-based Alzheimer's disease PRS increased risk of all these autoimmune diseases. In addition, the PRS was associated with reduced risk of cancers, which is a well-described beneficial side effect of reduced selftolerance commonly harnessed in cancers medications. ${ }^{27}$

Furthermore, risk factors, course of disease, and clinical picture in dementias and autoimmune diseases have several similarities. Alzheimer's and autoimmune diseases are more common in women $^{9,28,29}$ and the diseases share inflammatory risk factors such as smoking and obesity. ${ }^{9,23,30,31}$ Infections increase risk for autoimmune diseases, ${ }^{32}$ rate of cognitive decline, ${ }^{1}$ and dementias even 20 years before the diagnosis, ${ }^{7}$ and can acutely trigger rheumatoid arthritis, type 1 diabetes and the formation of autoantibodies against CNS that lead to tissue degeneration. ${ }^{8,9,11}$ Interestingly, many inflammation-related biomarkers in our analyses influenced pathways closely related to amyloid precursor protein, tau protein, or $\alpha$-synuclein suggesting that they may contribute to proteinopathies that characterize of Alzheimer's and Parkinson's disease and dementia with Levy bodies. This process has some similarities with AA form of amyloidosis in rheumatic arthritis in which uncontrolled inflammation accumulates amyloid to a range of tissues ${ }^{33}$ and with islet amyloidosis in diabetes that leads to tissue degeneration. ${ }^{34}$ Two MR studies suggested inflammatory risk factor may not be causally linked to dementias. ${ }^{35,36}$ However, those studies did not focus on processes that control self-tolerance and they may have used inflammatory markers that are only responses to these processes and thus prone to produce null finding or reverse causation in MR analyses.

\section{Common cellular mechanisms between autoimmune disease and dementias}

At cellular level, loss of central tolerance in central immune organs and subsequent escape of autoreactive B- and T-cells to periphery ${ }^{37,38}$ are thought to predispose to autoimmune diseases together with certain HLA alleles. ${ }^{9,39,40}$ Conversion to disease may occur after complex interplay between inflammation, infections, and tissue damage, with subsequent failure of peripheral selftolerance mechanisms such as ignorance, anergy, suppression, inhibition, and antigen presentation. ${ }^{37,38}$ Our results link all of these mechanism to the development of dementias. 
medRxiv preprint doi: https://doi.org/10.1101/2022.02.17.22271136; this version posted February 18, 2022. The copyright holder for this preprint (which was not certified by peer review) is the author/funder, who has granted medRxiv a license to display the preprint in

It is made available under a CC-BY-NC-ND 4.0 International license .

BBB normally controls tightly the access of toxins, pathogens, and peripheral molecules and cells to CNS. ${ }^{4}$ This keeps peripheral lymphocytes "ignorant" of CNS structures and prevents activation of autoreactive cells against CNS.$^{38}$ However, chronic antigen exposure and activation of innate and adaptive immune systems impair BBB by releasing pro-inflammatory cytokines, prostaglandins, and histamine leading to endothelial damage and upregulation of adhesion molecules. ${ }^{4,41}$ This exposes CNS to autoreactive peripheral lymphocytes that lose their ignorance. ${ }^{38}$ The prolonged inflammation also leads to reduced anergy, which allows more potentially self-reactive $\mathrm{T}$ lymphocytes to proliferate. ${ }^{38}$

Our MR analyses support these processes. We showed that higher plasma levels of the integral part of $\mathrm{BBB}$, the tight junction component $\left(\mathrm{TJP} 1^{42}\right.$ ), and proteins degrading it ( $\mathrm{AIMP} 1^{43}$ and $\mathrm{BIN} 1^{44}$ ), increased the risk of dementias. Moreover, pro-inflammatory C1Q, C1R, CD20, CD11, CDHR5, GPNMB, and IL1 $\beta^{45-49}$ elevated risk of dementias, whereas anti-inflammatory C4B, IL27, IL37, PTP1B, and SIGGIRR ${ }^{50-54}$ and barrier protecting IL- $17 \mathrm{~F}^{55}$ reduced the risk. In suppression and inhibition, regulatory T-cells suppress, or molecules on B- and T-cells inhibit activation of lymphocytes at peripheral checkpoints. ${ }^{38}$ Our MR analyses identified several checkpoint regulators including BAFFR, C1R, C1Q, CD11, CD19, CD20, CD22, CD33, CD40, CX3CR1, LTBR, PD-1, and PDL-1 ${ }^{37,48,56-64}$ as potential causal risk factors for poor cognitive performance and dementias, uncovering the importance of checkpoint control and autoreactive lymphocytes in dementias.

HLA analyses were also consistent with the autoimmunity hypothesis. After the increase in BBB permeability, peripherally driven neuroinflammation activates central neuroinflammatory responses in astrocytes and microglia. ${ }^{1,4}$ The chronic neuroinflammation, pathogens, and tissue damage initiate antigen presentation in HLA receptors on antigen-presenting cells in CNS that can activate the autoreactive B-and T-cells and initiate self-sustaining autoimmunity ${ }^{11,40}$ against CNS structures. Supporting this we identified 21 shared HLA-types between autoimmune diseases and dementias. Similarly, MR and pathway analyses supported causal status of HLA-DR and HLA-DQ cell types in dementias.

The autoreactive cells can be activated via bystander activation, or HLA-mediated molecular mimicry and epitope spreading. ${ }^{8-10,38}$ In bystander activation, sustained or excessive immune response can bypass normal T-cell antigen presentation to B-cells and activate autoreactive B-cell to plasma cell that produces autoantibodies. In molecular mimicry, autoreactive T- and B-cells first target foreign antigens, and then cross-react with similar self-antigens expressed by host cells. In epitope spreading, cryptic epitopes (parts of dying cells, and intrinsic or foreign DNA or RNA) normally invisible to immune system become visible after tissue damage, which activates autoreactive B- and T cells on these epitopes.

Our results also provide support for these mechanisms. In MR analyses, we observed that higher levels of naïve and memory CD20 B-cells, the main drug target for B-cell induced autoimmune diseases ${ }^{65}$ increases risk for Alzheimer's diseases. Similarly, higher levels of CD19, a co-receptor on B-cells that can lead to autoimmunity when hyperactivated ${ }^{66}$ increased Parkinson's diseases risk. We also observed that higher levels of IgG against Epstein-Barr virus' nuclear antigen, a marker of active infection that can mimic CNS structures, ${ }^{67,68}$ elevated Alzheimer's diseases risk. In 
medRxiv preprint doi: https://doi.org/10.1101/2022.02.17.22271136; this version posted February 18, 2022. The copyright holder for this preprint (which was not certified by peer review) is the author/funder, who has granted medRxiv a license to display the preprint in

It is made available under a CC-BY-NC-ND 4.0 International license .

addition, IFIT2 that intercepts foreign RNA and protects against central viral infections, ${ }^{69}$ reduced the risk of all dementia causing diseases except Parkinson's diseases. Moreover, the pathway analyses suggested that many proteins (COPS6, SOCS3, PSMB5, TOLLIP, TRIM21, PRPF40A, NOC4L, RPS6, IK, KRR1, and MTO1) ${ }^{70-80}$ involved in intracellular pathways from DNA, RNA, and protein processing to antigen presentation may be linked with amyloid $\beta$, tau protein, and $\alpha$ synuclein.

The identified 8 proteins with SNPs located near $A P O E$ gene were associated with increased BBB permeability, inflammation, neurotoxicity, and lipid transport disturbances, ${ }^{42,56,69,81-84}$ which are all processes linked to $A P O E$ in Alzheimer's disease. ${ }^{85}$ Our results suggest that the 8 proteins may be linked to the effects of $A P O E$. Another novel result was that higher levels of CD33, one of the top GWAS hits for Alzheimer's diseases ${ }^{86}$ and target receptor on microglia for an ongoing trial (NCT03822208) including mild to moderate Alzheimer's diseases patients, increased the risk of disease even when expressed on peripheral leukocytes.

\section{Anti-inflammatory medications and dementias}

Our IPW analyses on use of anti-inflammatory medications, including records of filled drug prescriptions in initially dementia-free individuals at baseline followed up to 20 years supported the modifiability of dysfunctional autoimmune processes. We replicated protective associations between TNF- $\alpha$ inhibitors, ${ }^{87}$ methotrexate. ${ }^{88}$ and Alzheimer's disease. As novel findings we showed that methotrexate may be protective only for Alzheimer's and Parkinson's disease among those with high MR-based PRS. These findings are plausible on a cellular level for BBB degradation and neuroinflammation. TNF- $\alpha$ is a gatekeeper of inflammatory responses and endothelial permeability. ${ }^{89}$ Methotrexate, in turn, modulates a range of inflammatory and immune responses in nearly every cell types forming the foundations for treatment of rheumatoid arthritis. ${ }^{9}$ NSAIDs and antihistamines appeared to decrease risk for all dementias but the validation using a negative control outcome (coronary heart disease) suggested that the IPW model for these medications was insufficient to replicate trial design.

Randomized controlled trials of TNF- $\alpha$ inhibitors in Alzheimer's disease patients have not shown benefits in 1 to 2 year follow-up. ${ }^{90,91}$ However, in autoimmune diseases, early initiation of antiinflammatory medication is of paramount importance suggesting that the trials of medication for people with established dementia may be too late. ${ }^{9}$

\section{Strengths and limitations}

Our triangulation approach has several strengths. By using a broad set of immune system and BBB biomarkers we were able to study these systems comprehensively without restrictions to specific predefined hypotheses. Our findings are based on MR, a promising approach to examine causality, ${ }^{12}$ and on analyses using plasma proteomics, PRS, HLA, pathway, and IPW analyses in two independent cohorts and a range of databases. Plasma protein analyses allowed us to adjust effect estimates for $A P O E$ which was not possible in Mendelian randomization analyses for some proteins. PRS and HLA analyses used data from the FinnGen study with a sample size of 340,000 providing sufficient statistical power for the identification of shared novel risk alleles between dementias and autoimmune disease. We used ConsensusPathDB, which is one of the most 
medRxiv preprint doi: https://doi.org/10.1101/2022.02.17.22271136; this version posted February 18, 2022. The copyright holder for this preprint (which was not certified by peer review) is the author/funder, who has granted medRxiv a license to display the preprint in

It is made available under a CC-BY-NC-ND 4.0 International license .

10

comprehensive collection of databases on molecular pathways and interactions. ${ }^{92}$ The medication analyses used IPW survival analysis that is suggested to provide more reliable causal estimates than traditional survival analyses of observational data. ${ }^{93}$

Our study has also several limitations. Although we explored over 40,000 biomarkers related to the immune system and BBB, we may have missed some biomarkers due to limitations in search protocol or lack of SNPs. Our plasma protein analyses were limited by lack of data on dementia subtypes. The PRS and PheWAS analyses were done with samples with European ancestry and may not apply to non-European ancestries. Due to limited number of SNPs included in PRS, we may have missed important immune system and BBB related associations in our phenome-wide analyses. Nonetheless, the Alzheimer's MR-based PRS associated strongly with all types of dementia (except Parkinson's disease) and a range of outcomes related to type 1 diabetes and rheumatic diseases suggesting that at least these findings are robust. The IPW analyses on medications may include some bias due to a limited number of covariates for simulation of randomized control trial. However, major bias is unlikely for the main findings since the analysis protocol was validated by a positive- and negative-control analyses.

\section{Conclusions}

In summary, this study provides novel insights into autoimmunity, BBB, and inflammatory dysfunctions that may contribute to the development of dementias. As the inflammatory components are potentially modifiable with anti-inflammatory medications, the present results also raise hypotheses on several novel drug targets for dementia-causing diseases which need to be validated in future experimental studies. Randomized controlled trials on early initiation of these medications for high-risk individuals are warranted while antigen-specific prevention strategies may offer new avenues in search of treatment for dementias.

\section{Methods}

\section{Mendelian randomization}

The SNPs for biomarkers and outcomes were searched from MR-base database. ${ }^{94}$ Immune system and BBB search terms were identified using identifiers of cell types, receptors, proteins, metabolites, and genes. The identifiers were searched from several publications $s^{3,4,6,23,36,38,40,95,96}$ and from Uniprot ${ }^{97}$ with search terms "immune" and "blood brain barrier". Outcomes included all cohorts with following diseases: all types of Alzheimer's disease, Parkinson's disease, vascular dementia, frontotemporal dementia, dementia in general, and progression of dementia. (SMethods). Poor cognitive performance was chosen as an intermediate outcome. Two sample MR was used to analyse associations between biomarkers and outcomes. ${ }^{12}$ First analyses estimated effects with Wald ratio or inverse variance-weighted analyses. ${ }^{94}$ For biomarker-outcome pairs that passed FDR of 5\% and shared over two common SNPs we performed sensitivity analyses with weighted 
medRxiv preprint doi: https://doi.org/10.1101/2022.02.17.22271136; this version posted February 18, 2022. The copyright holder for this preprint (which was not certified by peer review) is the author/funder, who has granted medRxiv a license to display the preprint in

It is made available under a CC-BY-NC-ND 4.0 International license .

11

median, simple mode, MR Egger, and backward Mendelian randomization ${ }^{12}$ with TwoSampleMR and MRInstruments R packages.

To assess off-target effects of the observed causal biomarkers, we performed a phenome-wide MR analysis for each biomarker, using GWAS summary statistics for 210 UK Biobank endpoints conducted by Neale lab and with recognized dementia risk factors. ${ }^{23,98}$

\section{Plasma protein analyses}

Plasma protein measurements in Whitehall II study were available for 6,235 individuals of whom 310 developed dementia and the methods have been describe before (SMethods). ${ }^{6,99,100}$ In brief, the plasma proteins were measured using the SomaScan version 4.0 and 4.1 assays. ${ }^{6,101,102}$ The assays were validated against external reference population and protein-specific conversion coefficients were used to balance the technical differences between versions 4.0 and 4.1. Using DNA extracted from whole blood, a standard PCR assay determined APOE genotype using the salting out method. ${ }^{103,104}$ In analyses, proteins were transformed to a normal distribution by inverse rank-based normal transformation. The analyses used plasma samples measured in 1995/1997 and the followup ended at onset of dementia, death, or $1^{\text {st }}$ of October 2019, which ever occurred first. Cox regression models adjusted for age, sex, and $A P O E$ estimated associations between the proteins and dementia. ${ }^{105}$ The proportionality assumption in all Cox models was assessed with Schoenfeld residuals and with log-log plots. ${ }^{105}$ We used statistical software R (3.6.0 and 4.1.0) for these analyses.

\section{Polygenic risk score and IPW analyses}

FinnGen Data Freeze 8 contains 339,233 individuals, and is a collection of prospective epidemiological and disease-based cohorts, and hospital biobank samples and links genotypes by the unique national personal identification numbers to nationwide health registries, including the national hospital discharge (available from 1968), death (1969-), cancer (1953-) and medication reimbursement (1964-) and purchase (1995-) registries. Genotyping and imputation is described in SMethods.

PRS was constructed from SNPs that were associated with the 126 MR identified causal biomarkers for dementias. The SNPs were LD-pruned with clumping cut-off $\mathrm{R}^{2}=0.01$ in 500kb window with $\mathrm{R}$ package TwoSampleMR. The available SNPs were searched from FinnGen genotypes and the final scores were generated with PLINK v2.00aLM3 by calculating the SNP-biomarker beta weighted sum of risk alleles for each SNP. The PRS was scaled to zero mean and unit variance, and we analysed its phenome-wide associations across 2401 disease endpoints. The association between PRS and endpoints were studied with logistic regression, adjusting for birth year, sex, and ten first principal components of ancestry.

The IPW analyse ${ }^{93}$ were done in FinnGen and included participants aged over 45 without any dementia-causing disease at baseline $(\mathrm{N}=90,512)$. ATC codes for anti-inflammatory medication use were obtained from linkage to national medication purchase registry (data starts from 1995) and 
medRxiv preprint doi: https://doi.org/10.1101/2022.02.17.22271136; this version posted February 18, 2022. The copyright holder for this preprint (which was not certified by peer review) is the author/funder, who has granted medRxiv a license to display the preprint in

It is made available under a CC-BY-NC-ND 4.0 International license .

12

in each analyses we included only new medication users to simulate trial design. ${ }^{106} \mathrm{~A}$ model including a range of baseline and time-varying variables and informative censoring estimated the weights (SMethods). The analyses used time-varying Cox models and assumptions were assessed similar to plasma proteins. The follow-up started in 1997 and ended at onset of dementia, death, or $31^{\text {st }}$ of December 2019, which ever occurred first. We used R (4.1.2) and ipw package for these analyses.

\section{HLA analyses}

The analyses were done using HLA alleles, imputed with high accuracy using a Finnish-specific reference panel, which has been previously described in detail. ${ }^{107}$ After filtering based on a HLA carrier frequency of $\geq 0.01$ and posterior probability of $\geq 0.6$, we assessed the association between HLA alleles and dementias and autoimmune diseases using logistic regression adjusted for birth year, sex, and ten first principal components of ancestry.

\section{Pathway analyses}

KEGG pathway analysis was done with Generally Applicable Gene-set Enrichment (GAGE), ${ }^{108}$ using MR betas and p-values as input for expression ratios. GO term enrichment analyses were done with ClueGO version 2.5.8 ${ }^{109}$ in Cytoscape version 3.7.2 ${ }^{110}$ (SMethods). The hypergeometric test used all the 76 plasma proteins or surface receptors of the 126 biomarkers as input and all immune system and BBB related proteins from Uniprot ${ }^{97}$ as background, with correction for $5 \%$ FDR. The shortest interaction path analyses were done with ConsensusPathDB, ${ }^{92}$ a web based analysis tool containing several biomedical databases.

\section{Open Targets analyses}

Medications that change the levels of the 126 biomarkers were searched from Open Targets database using Uniprot protein and Entrez gene symbols. 
medRxiv preprint doi: https://doi.org/10.1101/2022.02.17.22271136; this version posted February 18, 2022. The copyright holder for this preprint (which was not certified by peer review) is the author/funder, who has granted medRxiv a license to display the preprint in

It is made available under a CC-BY-NC-ND 4.0 International license .

13

\section{Ethics statement}

In the Whitehall II study, research ethics approvals were renewed at each wave; the most recent approval was obtained from the University College London Hospital Committee on the Ethics of Human Research (reference number 85/0938). Written, informed consent from participants was obtained at each contact.

Patients and control subjects in FinnGen provided informed consent for biobank research, based on the Finnish Biobank Act. Alternatively, separate research cohorts, collected prior the Finnish Biobank Act came into effect (in September 2013) and start of FinnGen (August 2017), were collected based on study-specific consents and later transferred to the Finnish biobanks after approval by Fimea (Finnish Medicines Agency), the National Supervisory Authority for Welfare and Health. Recruitment protocols followed the biobank protocols approved by Fimea. The Coordinating Ethics Committee of the Hospital District of Helsinki and Uusimaa (HUS) statement number for the FinnGen study is Nr HUS/990/2017.

The FinnGen study is approved by Finnish Institute for Health and Welfare (permit numbers: THL/2031/6.02.00/2017, THL/1101/5.05.00/2017, THL/341/6.02.00/2018, THL/2222/6.02.00/2018, THL/283/6.02.00/2019, THL/1721/5.05.00/2019 and THL/1524/5.05.00/2020), Digital and population data service agency (permit numbers: VRK43431/2017-3, VRK/6909/2018-3, VRK/4415/2019-3), the Social Insurance Institution (permit numbers: KELA 58/522/2017, KELA 131/522/2018, KELA 70/522/2019, KELA 98/522/2019, KELA 134/522/2019, KELA 138/522/2019, KELA 2/522/2020, KELA 16/522/2020), Findata permit numbers THL/2364/14.02/2020, THL/4055/14.06.00/2020,,THL/3433/14.06.00/2020, THL/4432/14.06/2020, THL/5189/14.06/2020, THL/5894/14.06.00/2020, THL/6619/14.06.00/2020, THL/209/14.06.00/2021, THL/688/14.06.00/2021, THL/1284/14.06.00/2021, THL/1965/14.06.00/2021, THL/5546/14.02.00/2020, THL/2658/14.06.00/2021, THL/4235/14.06.00/2021 and Statistics Finland (permit numbers: TK-53-1041-17 and TK/143/07.03.00/2020 (earlier TK-53-90-20) TK/1735/07.03.00/2021).

The Biobank Access Decisions for FinnGen samples and data utilized in FinnGen Data Freeze 8 include: THL Biobank BB2017_55, BB2017_111, BB2018_19, BB_2018_34, BB_2018_67, BB2018_71, BB2019_7, BB2019_8, BB2019_26, BB2020_1, Finnish Red Cross Blood Service Biobank 7.12.2017, Helsinki Biobank HUS/359/2017, Auria Biobank AB17-5154 and amendment \#1 (August 17 2020), AB20-5926 and amendment \#1 (April 23 2020), Biobank Borealis of Northern Finland_2017_1013, Biobank of Eastern Finland 1186/2018 and amendment 22 § /2020, Finnish Clinical Biobank Tampere MH0004 and amendments (21.02.2020 \& 06.10.2020), Central Finland Biobank 1-2017, and Terveystalo Biobank STB 2018001.

\section{Acknowledgments and Funding Sources}

We want to acknowledge the participants and investigators of FinnGen study. The FinnGen project is funded by two grants from Business Finland (HUS 4685/31/2016 and UH 4386/31/2016) and the following industry partners: AbbVie Inc., AstraZeneca UK Ltd, Biogen MA Inc., Bristol Myers Squibb (and Celgene Corporation \& Celgene International II Sàrl), Genentech Inc., Merck Sharp \& 
medRxiv preprint doi: https://doi.org/10.1101/2022.02.17.22271136; this version posted February 18, 2022. The copyright holder for this preprint (which was not certified by peer review) is the author/funder, who has granted medRxiv a license to display the preprint in

It is made available under a CC-BY-NC-ND 4.0 International license .

14

438 Dohme Corp, Pfizer Inc., GlaxoSmithKline Intellectual Property Development Ltd., Sanofi US

439 Services Inc., Maze Therapeutics Inc., Janssen Biotech Inc, Novartis AG, and Boehringer

440 Ingelheim. Following biobanks are acknowledged for delivering biobank samples to FinnGen:

441 Auria Biobank (www.auria.fi/biopankki), THL Biobank (www.thl.fi/biobank), Helsinki Biobank

442 (www.helsinginbiopankki.fi), Biobank Borealis of Northern Finland

443 (https://www.ppshp.fi/Tutkimus-ja-opetus/Biopankki/Pages/Biobank-Borealis-briefly-in-

444 English.aspx), Finnish Clinical Biobank Tampere (www.tays.fi/en-

445 US/Research_and_development/Finnish_Clinical_Biobank_Tampere), Biobank of Eastern Finland

446 (www.ita-suomenbiopankki.fi/en), Central Finland Biobank (www.ksshp.fi/fi-

447 FI/Potilaalle/Biopankki), Finnish Red Cross Blood Service Biobank

448 (www.veripalvelu.fi/verenluovutus/biopankkitoiminta) and Terveystalo Biobank

449 (www.terveystalo.com/fi/Yritystietoa/Terveystalo-Biopankki/Biopankki/). All Finnish Biobanks are 450 members of BBMRI.fi infrastructure (www.bbmri.fi). Finnish Biobank Cooperative -FINBB

451 (https://finbb.fi/) is the coordinator of BBMRI-ERIC operations in Finland. The Finnish biobank

452 data can be accessed through the Fingenious ${ }^{\circledR}$ services (https://site.fingenious.fi/en/) managed by

453 FINBB.

The study was supported by the Wellcome Trust (221854/Z/20/Z), the UK Medical Research Council (S011676, K013351, R024227), the National Institute on Aging (National Institutes of Health; R01AG056477 and RF1AG062553), the British Heart Foundation (RG/16/11/32334), the Academy of Finland (311492), NordForsk (75021), and SomaLogic, Inc. JVL was supported by Academy of Finland (339568). NM was supported by the Academy of Finland (331671). PNS was supported by the Emil Aaltonen Foundation. MK was supported by the Wellcome Trust (221854/Z/20/Z), UK Medical Research Council (MR/S011676, MR/R024227), US National Institute on Aging (R01AG062553, R01AG056477), Academy of Finland (311492), Helsinki Institute of Life Science (H970), and the Finnish Work Environment Fund (190424). SR was funded by Academy of Finland (285380 and 312062), Sigrid Jusélius Foundation and University of Helsinki HiLIFE Fellow grants 2017-2020.

FinnGen consortium authors are listed in FinnGen Banner Authors file.

\section{Author Contributions}

471 JVL, NM, SR, and MK generated the hypothesis and designed the study. JVL wrote the first draft of the report, did the primary analyses, with support from NM, and performed literature searches. All authors interpreted the data and critically commented and reviewed the report. JVL and MK had full access to pseudonymised data from the Whitehall II. JVL, NM, and SR had full access to pseudonymised data from the FinnGen study.

\section{Competing interests}

In Whitehall II study part of the proteins were measured as academic-industry partnership project beween UCL and SomaLogic, Inc. which provided expertise in plasma proteins and funded 2240 SOMAscan assays. PNS reports a grant from the Emil Aaltonen foundation, during the conduct of the study. MK reports grants from Wellcome Trust, UK Medical Research Council, US National 
medRxiv preprint doi: https://doi.org/10.1101/2022.02.17.22271136; this version posted February 18, 2022. The copyright holder for this preprint (which was not certified by peer review) is the author/funder, who has granted medRxiv a license to display the preprint in It is made available under a CC-BY-NC-ND 4.0 International license.

15

482 Institute on Aging, Academy of Finland, Helsinki Institute of Life Science, and the Finnish Work Environment Fund, during the conduct of the study.

\section{Data sharing}

Data, protocols, and other metadata of the Whitehall II and FinnGen studies are available according to the data sharing policies of these studies. Pre-existing data access policy for Whitehall II specify that research data requests can be submitted to each steering committee; these will be promptly reviewed for confidentiality or intellectual property restrictions and will not unreasonably be refused. Detailed information on data sharing can be found here: https://www.ucl.ac.uk/epidemiology-health-care/research/epidemiology-and-publichealth/research/whitehall-ii/data-sharing Individual-level patient or protein data may further be restricted by consent, confidentiality, or privacy laws/considerations. The FinnGen data may be accessed through Finnish Biobanks' FinBB portal (www.finbb.fi). 
medRxiv preprint doi: https://doi.org/10.1101/2022.02.17.22271136; this version posted February 18, 2022. The copyright holder for this preprint (which was not certified by peer review) is the author/funder, who has granted medRxiv a license to display the preprint in It is made available under a CC-BY-NC-ND 4.0 International license .

\section{References}

1. Bettcher, B.M., Tansey, M.G., Dorothée, G. \& Heneka, M.T. Peripheral and central immune system crosstalk in Alzheimer disease - a research prospectus. Nat Rev Neurol 17, 689-701 (2021).

2. Kinney, J.W., et al. Inflammation as a central mechanism in Alzheimer's disease. Alzheimers Dement (N Y) 4, 575-590 (2018).

3. Tan, E.K., et al. Parkinson disease and the immune system - associations, mechanisms and therapeutics. Nat Rev Neurol 16, 303-318 (2020).

4. Sweeney, M.D., Zhao, Z., Montagne, A., Nelson, A.R. \& Zlokovic, B.V. Blood-Brain Barrier: From Physiology to Disease and Back. Physiological Reviews 99, 21-78 (2019).

5. Franceschi, C., Garagnani, P., Parini, P., Giuliani, C. \& Santoro, A. Inflammaging: a new immune-metabolic viewpoint for age-related diseases. Nat Rev Endocrinol 14, 576-590 (2018).

6. Lindbohm, J.V., et al. Plasma proteins, cognitive decline, and 20-year risk of dementia in the Whitehall II and Atherosclerosis Risk in Communities studies. Alzheimer's \& dementia : the journal of the Alzheimer's Association (2021).

7. Sipilä, P.N., et al. Hospital-treated infectious diseases and the risk of dementia: a large, multicohort, observational study with a replication cohort. Lancet Infect Dis 21, 1557-1567 (2021).

8. Op de Beeck, A. \& Eizirik, D.L. Viral infections in type 1 diabetes mellitus--why the $\beta$ cells? Nat Rev Endocrinol 12, 263-273 (2016).

9. Smolen, J.S., et al. Rheumatoid arthritis. Nature Reviews Disease Primers 4, 18001 (2018).

10. Stassi, G. \& De Maria, R. Autoimmune thyroid disease: new models of cell death in autoimmunity. Nat Rev Immunol 2, 195-204 (2002).

11. Prüss, H. Autoantibodies in neurological disease. Nat Rev Immunol 21, 798-813 (2021).

12. Holmes, M.V., Richardson, T.G., Ference, B.A., Davies, N.M. \& Davey Smith, G. Integrating genomics with biomarkers and therapeutic targets to invigorate cardiovascular drug development. Nat Rev Cardiol 18, 435-453 (2021).

13. Munafo, M.R. \& Davey Smith, G. Robust research needs many lines of evidence. Nature 553, 399-401 (2018).

14. Law, M.R., Wald, N.J. \& Rudnicka, A.R. Quantifying effect of statins on low density lipoprotein cholesterol, ischaemic heart disease, and stroke: systematic review and metaanalysis. Bmj 326, 1423 (2003).

15. Bally, M., et al. Risk of acute myocardial infarction with NSAIDs in real world use: bayesian meta-analysis of individual patient data. Bmj 357, j1909 (2017).

16. Thanigaimani, S., Phie, J., Krishna, S.M., Moxon, J. \& Golledge, J. Effect of disease modifying anti-rheumatic drugs on major cardiovascular events: a meta-analysis of randomized controlled trials. Sci Rep 11, 6627 (2021).

17. Ridker, P.M., et al. Low-Dose Methotrexate for the Prevention of Atherosclerotic Events. New England Journal of Medicine 380, 752-762 (2018).

18. Broce, I., et al. Immune-related genetic enrichment in frontotemporal dementia: An analysis of genome-wide association studies. PLoS Med 15, e1002487 (2018).

19. Chou, R.C., Kane, M., Ghimire, S., Gautam, S. \& Gui, J. Treatment for Rheumatoid Arthritis and Risk of Alzheimer's Disease: A Nested Case-Control Analysis. CNS Drugs 30, 1111-1120 (2016).

20. Wallin, K., et al. Midlife rheumatoid arthritis increases the risk of cognitive impairment two decades later: a population-based study. J Alzheimers Dis 31, 669-676 (2012). 
medRxiv preprint doi: https://doi.org/10.1101/2022.02.17.22271136; this version posted February 18, 2022. The copyright holder for this preprint (which was not certified by peer review) is the author/funder, who has granted medRxiv a license to display the preprint in

It is made available under a CC-BY-NC-ND 4.0 International license .

17

21. Zhou, M., Xu, R., Kaelber, D.C. \& Gurney, M.E. Tumor Necrosis Factor (TNF) blocking agents are associated with lower risk for Alzheimer's disease in patients with rheumatoid arthritis and psoriasis. PLoS One 15, e0229819 (2020).

22. Li, X., Sundquist, J. \& Sundquist, K. Subsequent risks of Parkinson disease in patients with autoimmune and related disorders: a nationwide epidemiological study from Sweden. Neurodegener Dis 10, 277-284 (2012).

23. Livingston, G., et al. Dementia prevention, intervention, and care: 2020 report of the Lancet Commission. Lancet (London, England) 396, 413-446 (2020).

24. Jacobson, A.M., et al. Cognitive performance declines in older adults with type 1 diabetes: results from 32 years of follow-up in the DCCT and EDIC Study. Lancet Diabetes Endocrinol 9, 436-445 (2021).

25. Lacy, M.E., et al. Long-term Glycemic Control and Dementia Risk in Type 1 Diabetes. Diabetes Care 41, 2339-2345 (2018).

26. Sipilä, P.N., et al. Long-term risk of dementia following hospitalization due to physical diseases: A multicohort study. Alzheimer's \& dementia : the journal of the Alzheimer's Association 16, 1686-1695 (2020).

27. Leach, D.R., Krummel, M.F. \& Allison, J.P. Enhancement of antitumor immunity by CTLA-4 blockade. Science 271, 1734-1736 (1996).

28. Krischer, J.P., et al. The Influence of Type 1 Diabetes Genetic Susceptibility Regions, Age, Sex, and Family History on the Progression From Multiple Autoantibodies to Type 1 Diabetes: A TEDDY Study Report. Diabetes 66, 3122-3129 (2017).

29. Gleicher, N. \& Barad, D.H. Gender as risk factor for autoimmune diseases. J Autoimmun 28, 1-6 (2007).

30. Corbin, K.D., et al. Obesity in Type 1 Diabetes: Pathophysiology, Clinical Impact, and Mechanisms. Endocr Rev 39, 629-663 (2018).

31. Rasouli, B., et al. Smoking and the Risk of LADA: Results From a Swedish PopulationBased Case-Control Study. Diabetes Care 39, 794-800 (2016).

32. Nielsen, P.R., Kragstrup, T.W., Deleuran, B.W. \& Benros, M.E. Infections as risk factor for autoimmune diseases - A nationwide study. J Autoimmun 74, 176-181 (2016).

33. Perfetto, F., et al. Systemic amyloidosis: a challenge for the rheumatologist. Nature Reviews Rheumatology 6, 417-429 (2010).

34. Rojas, J., et al. Pancreatic Beta Cell Death: Novel Potential Mechanisms in Diabetes Therapy. J Diabetes Res 2018, 9601801 (2018).

35. Fani, L., et al. Circulating biomarkers of immunity and inflammation, risk of Alzheimer's disease, and hippocampal volume: a Mendelian randomization study. Translational Psychiatry 11, 291 (2021).

36. Walker, K.A., et al. Large-scale plasma proteomic analysis identifies proteins and pathways associated with dementia risk. Nature Aging 1, 473-489 (2021).

37. von Boehmer, H. \& Melchers, F. Checkpoints in lymphocyte development and autoimmune disease. Nat Immunol 11, 14-20 (2010).

38. Theofilopoulos, A.N., Kono, D.H. \& Baccala, R. The multiple pathways to autoimmunity. Nat Immunol 18, 716-724 (2017).

39. Buzzetti, R., Zampetti, S. \& Maddaloni, E. Adult-onset autoimmune diabetes: current knowledge and implications for management. Nat Rev Endocrinol 13, 674-686 (2017).

40. Dendrou, C.A., Petersen, J., Rossjohn, J. \& Fugger, L. HLA variation and disease. Nat Rev Immunol 18, 325-339 (2018).

41. Abbott, N.J. Inflammatory mediators and modulation of blood-brain barrier permeability. Cell Mol Neurobiol 20, 131-147 (2000).

42. Furuse, M., et al. Direct association of occludin with ZO-1 and its possible involvement in the localization of occludin at tight junctions. J Cell Biol 127, 1617-1626 (1994). 
medRxiv preprint doi: https://doi.org/10.1101/2022.02.17.22271136; this version posted February 18, 2022. The copyright holder for this preprint (which was not certified by peer review) is the author/funder, who has granted medRxiv a license to display the preprint in It is made available under a CC-BY-NC-ND 4.0 International license .

43. Li, C., Ma, W., Zhao, Y. \& Wang, H. Changes in the expression of endothelial monocyte-activating polypeptide II in the rat hippocampus following status epilepticus. Int $J$ Mol Med 47, 699-707 (2021).

44. Thomas, S., et al. Novel Colitis Immunotherapy Targets Bin1 and Improves Colon Cell Barrier Function. Dig Dis Sci 61, 423-432 (2016).

45. Carmona, F.D., et al. Novel identification of the IRF7 region as an anticentromere autoantibody propensity locus in systemic sclerosis. Ann Rheum Dis 71, 114-119 (2012).

46. Dalakas, M.C., Alexopoulos, H. \& Spaeth, P.J. Complement in neurological disorders and emerging complement-targeted therapeutics. Nat Rev Neurol 16, 601-617 (2020).

47. Migliorini, P., Italiani, P., Pratesi, F., Puxeddu, I. \& Boraschi, D. The IL-1 family cytokines and receptors in autoimmune diseases. Autoimmun Rev 19, 102617 (2020).

48. Nowatzky, J., Manches, O., Khan, S.A., Godefroy, E. \& Bhardwaj, N. Modulation of human Th17 cell responses through complement receptor 3 (CD11 b/CD18) ligation on monocytederived dendritic cells. J Autoimmun 92, 57-66 (2018).

49. Saade, M., Araujo de Souza, G., Scavone, C. \& Kinoshita, P.F. The Role of GPNMB in Inflammation. Front Immunol 12, 674739 (2021).

50. Berdnikovs, S., et al. PTP1B deficiency exacerbates inflammation and accelerates leukocyte trafficking in vivo. J Immunol 188, 874-884 (2012).

51. Kamitaki, N., et al. Complement genes contribute sex-biased vulnerability in diverse disorders. Nature 582, 577-581 (2020).

52. Meka, R.R., Venkatesha, S.H., Dudics, S., Acharya, B. \& Moudgil, K.D. IL-27-induced modulation of autoimmunity and its therapeutic potential. Autoimmun Rev 14, 1131-1141 (2015).

53. Xu, W.D., Zhao, Y. \& Liu, Y. Insights into IL-37, the role in autoimmune diseases. Autoimmun Rev 14, 1170-1175 (2015).

54. Cavalli, G. \& Dinarello, C.A. Suppression of inflammation and acquired immunity by IL37. Immunol Rev 281, 179-190 (2018).

55. McGeachy, M.J., Cua, D.J. \& Gaffen, S.L. The IL-17 Family of Cytokines in Health and Disease. Immunity 50, 892-906 (2019).

56. Brink, R. Regulation of B cell self-tolerance by BAFF. Semin Immunol 18, 276-283 (2006).

57. Choi, J.W., et al. The prognostic significance of VISTA and CD33-positive myeloid cells in cutaneous melanoma and their relationship with PD-1 expression. Scientific Reports 10, 14372 (2020).

58. Wang, Q., et al. Transient BAFF Blockade Inhibits Type 1 Diabetes Development in Nonobese Diabetic Mice by Enriching Immunoregulatory B Lymphocytes Sensitive to Deletion by Anti-CD20 Cotherapy. J Immunol 199, 3757-3770 (2017).

59. Yamauchi, T., et al. T-cell CX3CR1 expression as a dynamic blood-based biomarker of response to immune checkpoint inhibitors. Nature Communications 12, 1402 (2021).

60. Dörner, T., Shock, A. \& Smith, K.G. CD22 and autoimmune disease. Int Rev Immunol 31, 363-378 (2012).

61. Gommerman, J.L. \& Summers deLuca, L. LT $\beta R$ and CD40: working together in dendritic cells to optimize immune responses. Immunol Rev 244, 85-98 (2011).

62. Maldini, C.R., Ellis, G.I. \& Riley, J.L. CAR T cells for infection, autoimmunity and allotransplantation. Nat Rev Immunol 18, 605-616 (2018).

63. Schittenhelm, L., Hilkens, C.M. \& Morrison, V.L. $\beta(2)$ Integrins As Regulators of Dendritic Cell, Monocyte, and Macrophage Function. Front Immunol 8, 1866 (2017).

64. Wang, J. \& Fu, Y.X. The role of LIGHT in T cell-mediated immunity. Immunol Res 30, 201-214 (2004). 
medRxiv preprint doi: https://doi.org/10.1101/2022.02.17.22271136; this version posted February 18, 2022. The copyright holder for this preprint (which was not certified by peer review) is the author/funder, who has granted medRxiv a license to display the preprint in

It is made available under a CC-BY-NC-ND 4.0 International license .

19

65. Meffre, E. \& O'Connor, K.C. Impaired B-cell tolerance checkpoints promote the development of autoimmune diseases and pathogenic autoantibodies. Immunol Rev 292, 90101 (2019).

66. Hobeika, E., Nielsen, P.J. \& Medgyesi, D. Signaling mechanisms regulating B-lymphocyte activation and tolerance. J Mol Med (Berl) 93, 143-158 (2015).

67. Gate, D., et al. Clonally expanded CD8 T cells patrol the cerebrospinal fluid in Alzheimer's disease. Nature 577, 399-404 (2020).

68. Xiao, D., et al. A meta-analysis of interaction between Epstein-Barr virus and HLADRB1*1501 on risk of multiple sclerosis. Scientific Reports 5, 18083 (2015).

69. Das Sarma, J., et al. Ifit2 deficiency restricts microglial activation and leukocyte migration following murine coronavirus (m-CoV) CNS infection. PLoS Pathog 16, e1009034 (2020).

70. Bech-Otschir, D., et al. COP9 signalosome-specific phosphorylation targets p53 to degradation by the ubiquitin system. Embo $j$ 20, 1630-1639 (2001).

71. Bertram, K., et al. Cryo-EM Structure of a Pre-catalytic Human Spliceosome Primed for Activation. Cell 170, 701-713.e711 (2017).

72. Choudhary, B., Marx, O. \& Norris, A.D. Spliceosomal component PRP-40 is a central regulator of microexon splicing. Cell Rep 36, 109464 (2021).

73. Groettrup, M., et al. A role for the proteasome regulator PA28alpha in antigen presentation. Nature 381, 166-168 (1996).

74. Kamura, T., et al. VHL-box and SOCS-box domains determine binding specificity for Cul2Rbx1 and Cul5-Rbx2 modules of ubiquitin ligases. Genes Dev 18, 3055-3065 (2004).

75. Lu, K., Psakhye, I. \& Jentsch, S. Autophagic clearance of polyQ proteins mediated by ubiquitin-Atg8 adaptors of the conserved CUET protein family. Cell 158, 549-563 (2014).

76. Sturm, M., Cheng, J., Baßler, J., Beckmann, R. \& Hurt, E. Interdependent action of KH domain proteins Krr1 and Dim2 drive the 40S platform assembly. Nat Commun 8, 2213 (2017).

77. Wada, K. \& Kamitani, T. Autoantigen Ro52 is an E3 ubiquitin ligase. Biochem Biophys Res Commun 339, 415-421 (2006).

78. Yamashita, D., et al. $\mathrm{hDREF}$ regulates cell proliferation and expression of ribosomal protein genes. Mol Cell Biol 27, 2003-2013 (2007).

79. Zhu, X., et al. Noc4L-Mediated Ribosome Biogenesis Controls Activation of Regulatory and Conventional T Cells. Cell Rep 27, 1205-1220.e1204 (2019).

80. Li, X., Li, R., Lin, X. \& Guan, M.X. Isolation and characterization of the putative nuclear modifier gene MTO1 involved in the pathogenesis of deafness-associated mitochondrial 12 S rRNA A1555G mutation. J Biol Chem 277, 27256-27264 (2002).

81. Estefanía, E., et al. Human KIR2DL5 is an inhibitory receptor expressed on the surface of NK and T lymphocyte subsets. J Immunol 178, 4402-4410 (2007).

82. Adly, A.A.M., Ismail, E.A., Tawfik, L.M., Ebeid, F.S.E. \& Hassan, A.A.S. Endothelial monocyte activating polypeptide II in children and adolescents with type 1 diabetes mellitus: Relation to micro-vascular complications. Cytokine 76, 156-162 (2015).

83. Dolan, J., et al. The extracellular leucine-rich repeat superfamily; a comparative survey and analysis of evolutionary relationships and expression patterns. BMC Genomics 8, 320 (2007).

84. García-Heredia, J.M. \& Carnero, A. The cargo protein MAP17 (PDZK1IP1) regulates the immune microenvironment. Oncotarget 8, 98580-98597 (2017).

85. Yamazaki, Y., Zhao, N., Caulfield, T.R., Liu, C.C. \& Bu, G. Apolipoprotein E and Alzheimer disease: pathobiology and targeting strategies. Nat Rev Neurol 15, 501-518 (2019).

86. Naj, A.C., et al. Common variants at MS4A4/MS4A6E, CD2AP, CD33 and EPHA1 are associated with late-onset Alzheimer's disease. Nat Genet 43, 436-441 (2011). 
medRxiv preprint doi: https://doi.org/10.1101/2022.02.17.22271136; this version posted February 18, 2022. The copyright holder for this preprint (which was not certified by peer review) is the author/funder, who has granted medRxiv a license to display the preprint in

It is made available under a CC-BY-NC-ND 4.0 International license .

20

87. Torres-Acosta, N., O'Keefe, J.H., O'Keefe, E.L., Isaacson, R. \& Small, G. Therapeutic Potential of TNF- $\alpha$ Inhibition for Alzheimer's Disease Prevention. J Alzheimers Dis 78, 619626 (2020).

88. Judge, A., et al. Protective effect of antirheumatic drugs on dementia in rheumatoid arthritis patients. Alzheimers Dement (N Y) 3, 612-621 (2017).

89. Chen, A.Y., Wolchok, J.D. \& Bass, A.R. TNF in the era of immune checkpoint inhibitors: friend or foe? Nature Reviews Rheumatology 17, 213-223 (2021).

90. Aisen, P.S., et al. Effects of Rofecoxib or Naproxen vs Placebo on Alzheimer Disease ProgressionA Randomized Controlled Trial. JAMA 289, 2819-2826 (2003).

91. Butchart, J., et al. Etanercept in Alzheimer disease: A randomized, placebo-controlled, double-blind, phase 2 trial. Neurology 84, 2161-2168 (2015).

92. Herwig, R., Hardt, C., Lienhard, M. \& Kamburov, A. Analyzing and interpreting genome data at the network level with ConsensusPathDB. Nature Protocols 11, 1889-1907 (2016).

93. Hernán, M.A. \& Robins, J.M. Using Big Data to Emulate a Target Trial When a Randomized Trial Is Not Available. Am J Epidemiol 183, 758-764 (2016).

94. Hemani, G., et al. The MR-Base platform supports systematic causal inference across the human phenome. Elife 7(2018).

95. Akiyama, H., et al. Inflammation and Alzheimer's disease. Neurobiology of aging 21, 383$421(2000)$.

96. O'Brien, J.T. \& Thomas, A. Vascular dementia. Lancet (London, England) 386, 1698-1706 (2015).

97. UniProt, C. UniProt: a worldwide hub of protein knowledge. Nucleic acids research 47, D506-D515 (2019).

98. http://www.nealelab.is/uk-biobank/.

99. Kivimaki, M., et al. Validity of Cardiovascular Disease Event Ascertainment Using Linkage to UK Hospital Records. Epidemiology (Cambridge, Mass.) 28, 735-739 (2017).

100. Sommerlad, A., et al. Accuracy of general hospital dementia diagnoses in England:

Sensitivity, specificity, and predictors of diagnostic accuracy 2008-2016. Alzheimer's \& dementia : the journal of the Alzheimer's Association 14, 933-943 (2018).

101. Gold, L., et al. Aptamer-based multiplexed proteomic technology for biomarker discovery. PloS one 5, e15004 (2010).

102. Williams, S.A., et al. Plasma protein patterns as comprehensive indicators of health. Nature medicine 25, 1851-1857 (2019).

103. Bolla, M.K., Wood, N. \& Humphries, S.E. Rapid determination of apolipoprotein E genotype using a heteroduplex generator. Journal of lipid research 40, 2340-2345 (1999).

104. Miller, S.A., Dykes, D.D. \& Polesky, H.F. A simple salting out procedure for extracting DNA from human nucleated cells. Nucleic acids research 16, 1215 (1988).

105. Cox, D.R. Regression Models and Life-Tables. Journal of the Royal Statistical Society.Series B (Methodological) 34, 187-220 (1972).

106. Dickerman, B.A., García-Albéniz, X., Logan, R.W., Denaxas, S. \& Hernán, M.A. Avoidable flaws in observational analyses: an application to statins and cancer. Nat Med 25, 1601-1606 (2019).

107. Ritari, J., Hyvärinen, K., Clancy, J., Partanen, J. \& Koskela, S. Increasing accuracy of HLA imputation by a population-specific reference panel in a FinnGen biobank cohort. NAR Genom Bioinform 2, lqaa030 (2020).

108. Luo, W., Friedman, M.S., Shedden, K., Hankenson, K.D. \& Woolf, P.J. GAGE: generally applicable gene set enrichment for pathway analysis. BMC Bioinformatics 10, 161 (2009).

109. Bindea, G., et al. ClueGO: a Cytoscape plug-in to decipher functionally grouped gene ontology and pathway annotation networks. Bioinformatics 25, 1091-1093 (2009). 
medRxiv preprint doi: https://doi.org/10.1101/2022.02.17.22271136; this version posted February 18, 2022. The copyright holder for this preprint (which was not certified by peer review) is the author/funder, who has granted medRxiv a license to display the preprint in

It is made available under a CC-BY-NC-ND 4.0 International license .

21

772 110. Shannon, P., et al. Cytoscape: a software environment for integrated models of biomolecular 773 774 interaction networks. Genome Res 13, 2498-2504 (2003).

775

776

777

778 
Figure 1. Flowchart of the study design. HLA, human leukocyte antigen; KEGG, Kyoto Encyclopedia of Genes and Genomes; PRS, polygenic risk score; SNP, single nucleotide polymorphism.

\section{1) Biomarker discovery step: two-sample Mendelian randomization analyses}

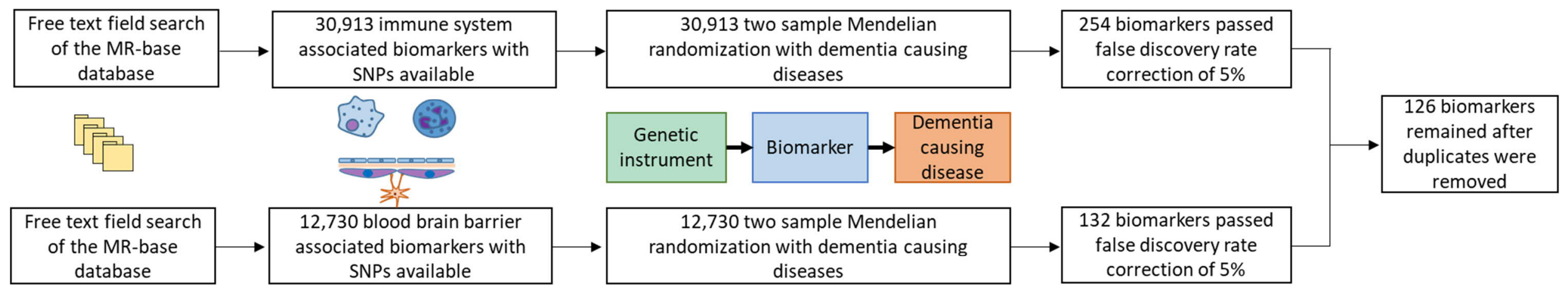

\section{2) Validation step for the 126 biomarkers: triangulation analyses}

A) Mendelian randomization sensitivity analyses with weighted median, simple mode, Egger and backward Mendelian randomization methods using MR-base
B) Polygenic risk score analyses for the SNPs that change levels of the biomarkers using

FinnGen $\mathrm{N}=339,233$

\section{C) HLA analyses to identify alleles that increase risk for dementias using FinnGen $\mathrm{N}=339,233$}

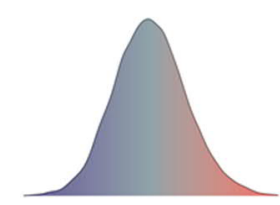

D) Plasma protein analyses to differentiate effect of strong risk effect alleles using Whitehall II $\mathrm{N}=6,235$

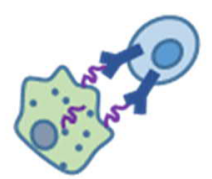

E) Pathway analyses
on molecular
functions of the
biomarkers using
KEGG, ClueGO, and
ConsensusPathDB
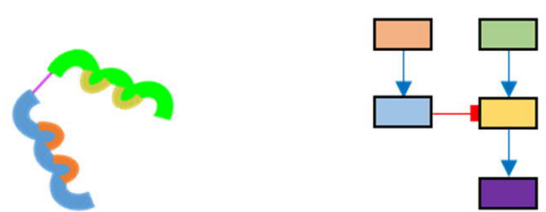

F) Inverse probability weighted analyses for medications that change the biomarkers levels using FinnGen $\mathrm{N}=90,512$
Observed Counterfactual

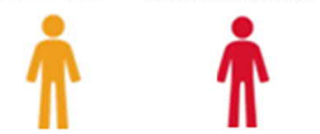


Figure 2. The autoimmune hypothesis of diseases causing dementias, the supporting evidence from current analyses are listed at each mechanism. BBB, blood brain barrier; CD, cluster of differentiation; CNS, central nervoussystem; HLA, Human Leukocyte Antigen; cluster of differentiation; IPW, inverse probability weighted; 
Central immune organs

\section{Thymus}

Autoreactive CD4 and

1) Increased production of primed mature
autoreactive $B$-cells and $C D 4$ and $C D 8 T$-cells due to failed positive and negative selection

in bone marrow and thymus.

\section{Supporting evidence from:}

Mendelian randomization

Polygenic risk scores

HLA analyses

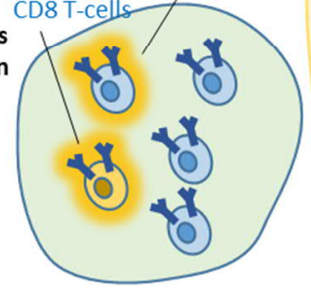

Bone marrow

Pathway analyses

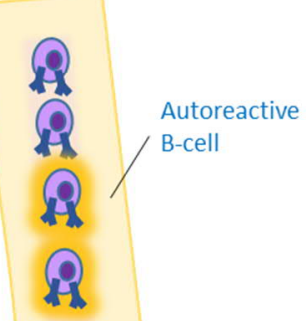

\section{Capillary}

2) Chronic antigen stimulation and increased

inflammation reduces anergy and allows more

autoreactive cells to proliferate. It also degrades

BBB that leads to contact between autoreactive

peripheral and CNS epitopes.

Supporting evidence from:

Mendelian randomization

Plasma proteomics

IPW analyses

Pathway analyses

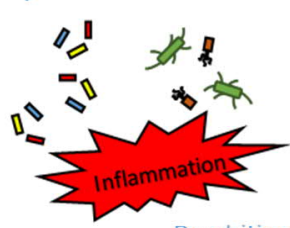

Secondary immune organs

Lymph node

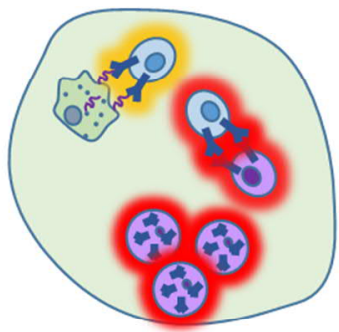

4) Antigen-presenting cells migrate to lymph nodes and activate autoreactive CD4 T-helper cells that activate autoreactive B-cells to plasma cells that secrete autoantibodies.

\section{Supporting evidence from:}

Mendelian randomization

HLA analyses

Pathway analyses
Lymph node

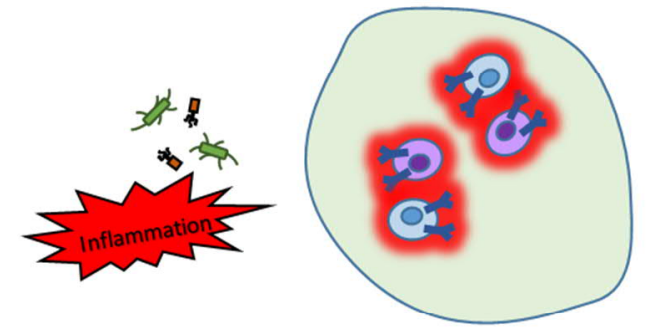

6) Memory cells for autoantigens are formed and they react to reoccurring inflammation, tissue damage and infections. This sustains autoimmune driven

neuroinflammation that causes permanent damage to CNS.

\section{Supporting evidence from:}

Mendelian randomization

Plasma proteomics

IPW analyses

Pathway analyses

Blood brain barrier Cytokines 0 Macrophage Neutroph

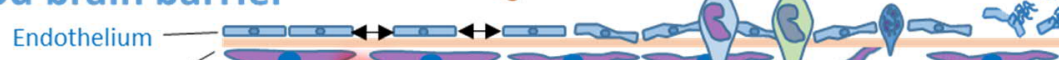

Basement

Membrane Pericytes

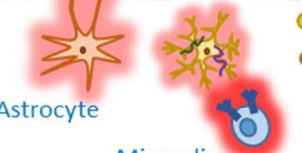

0

0

Central nervous system

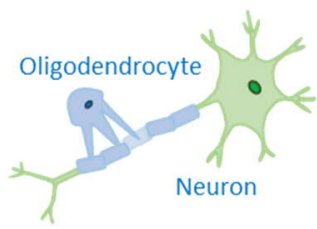

\section{0 落}

3) Inflammation, tissue damage, and

infections activate autoreactive $B$ - and

T-cells via bystander activation or

antigen presentation mediated

molecular mimicry and cryptic

epitopes. Autoreactive CD8-cells attack

CNS cells. Inflammation increases

amyloid production.

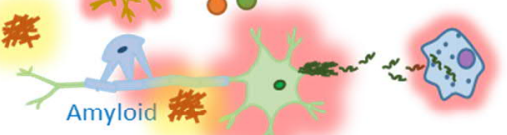

Supporting evidence from:

Mendelian randomization

Plasma proteomics

HLA analyses

Pathway analyses

IPW analyses

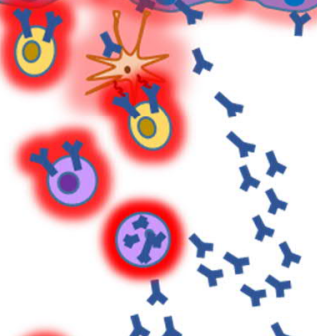

5) Autoantibodies and peripheral leukocytes infiltrate CNS, activate microglia, and sustain neuroinflammation. This leads to

neurodegeneration and formation of amyloid $\beta$, tau protein, and $\alpha$ synuclein that further increase neuroinflammation.

porting evidence from

Mendelian randomization

Plasma proteomics

IPW analyses

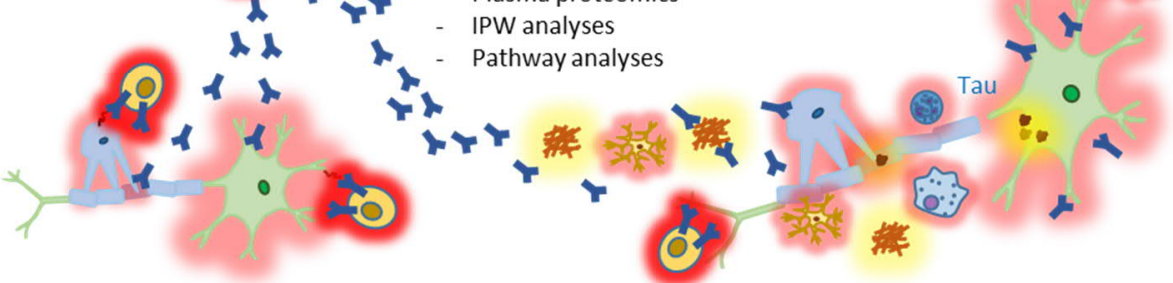


Figure 3A. Wald ratio or inverse variance weighted Mendelian randomization estimates for outcome-specific biomarkers. 


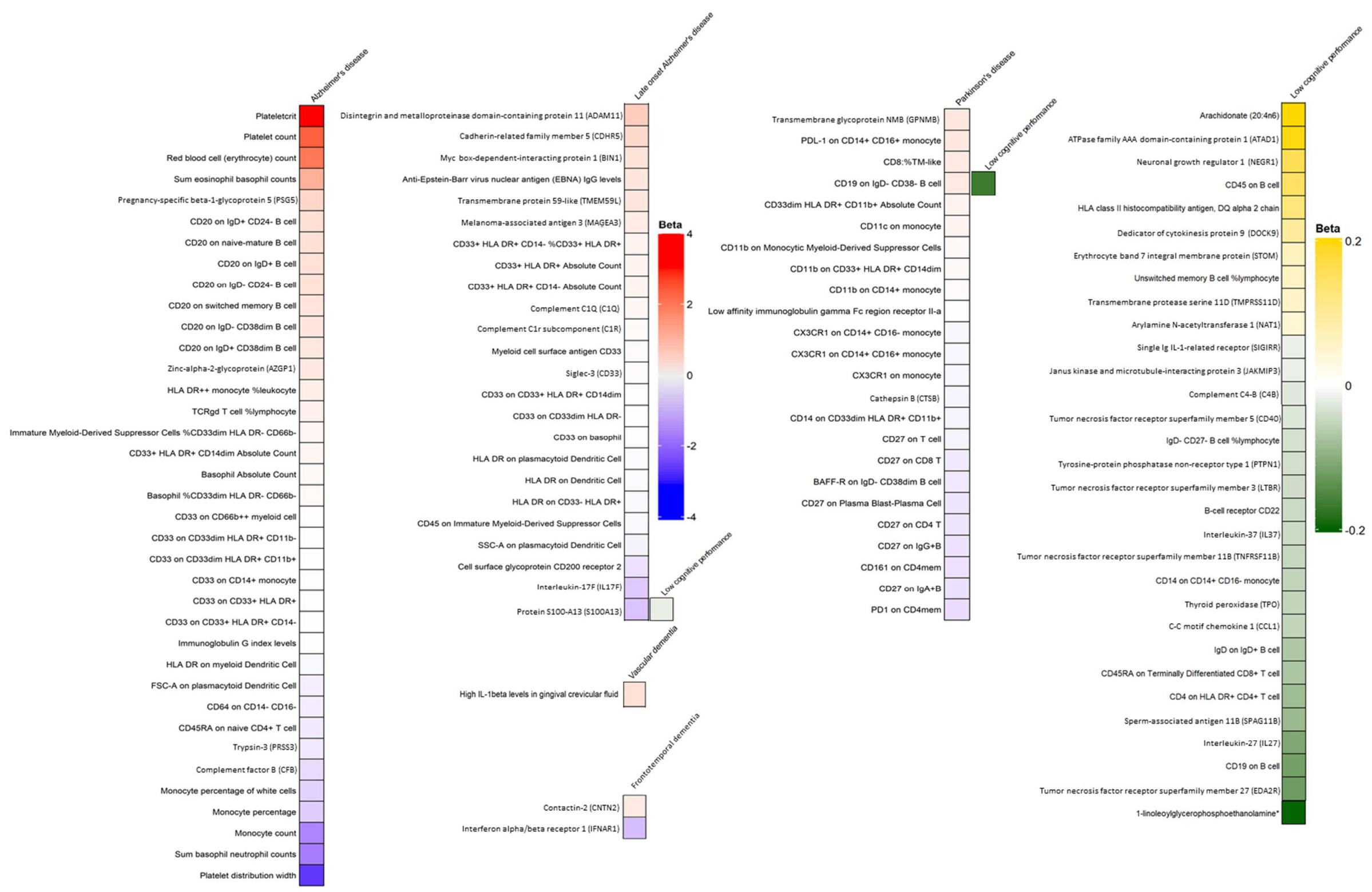


medRxiv preprint doi: https://doi.org/10.1101/2022.02.17.22271136; this version posted February 18, 2022. The copyright holder for this preprint (which was not certified by peer review) is the author/funder, who has granted medRxiv a license to display the preprint in

It is made available under a CC-BY-NC-ND 4.0 International license .

\section{7}

Figure 3B. Wald ratio or inverse variance weighted Mendelian randomization estimate for biomarkers that associated with at least three outcomes. These biomarkers localized around APOE gene. Biomarkers outcome pairs with no common SNPs available are on grey colour.

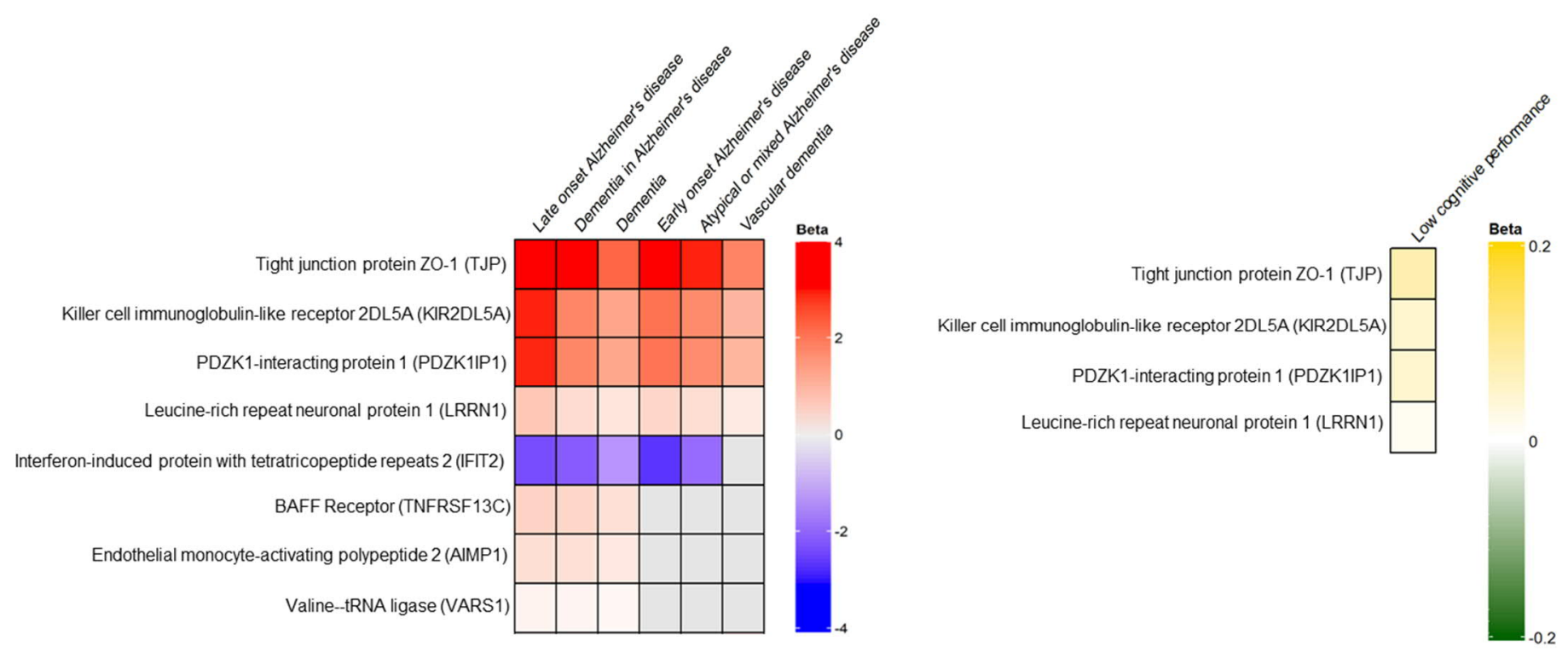


Figure 4. Phenome-wide association analyses for a Mendelian randomization-based polygenic risk score constructed only from SNPs that associated with levels of causal Alzheimer's disease biomarkers in Mendelian randomization Wald ratio or inverse variance weighted analyses. Upward pointing triangle means risk increasing and downward risk decreasing. Larger triangle indicates larger effect size. 

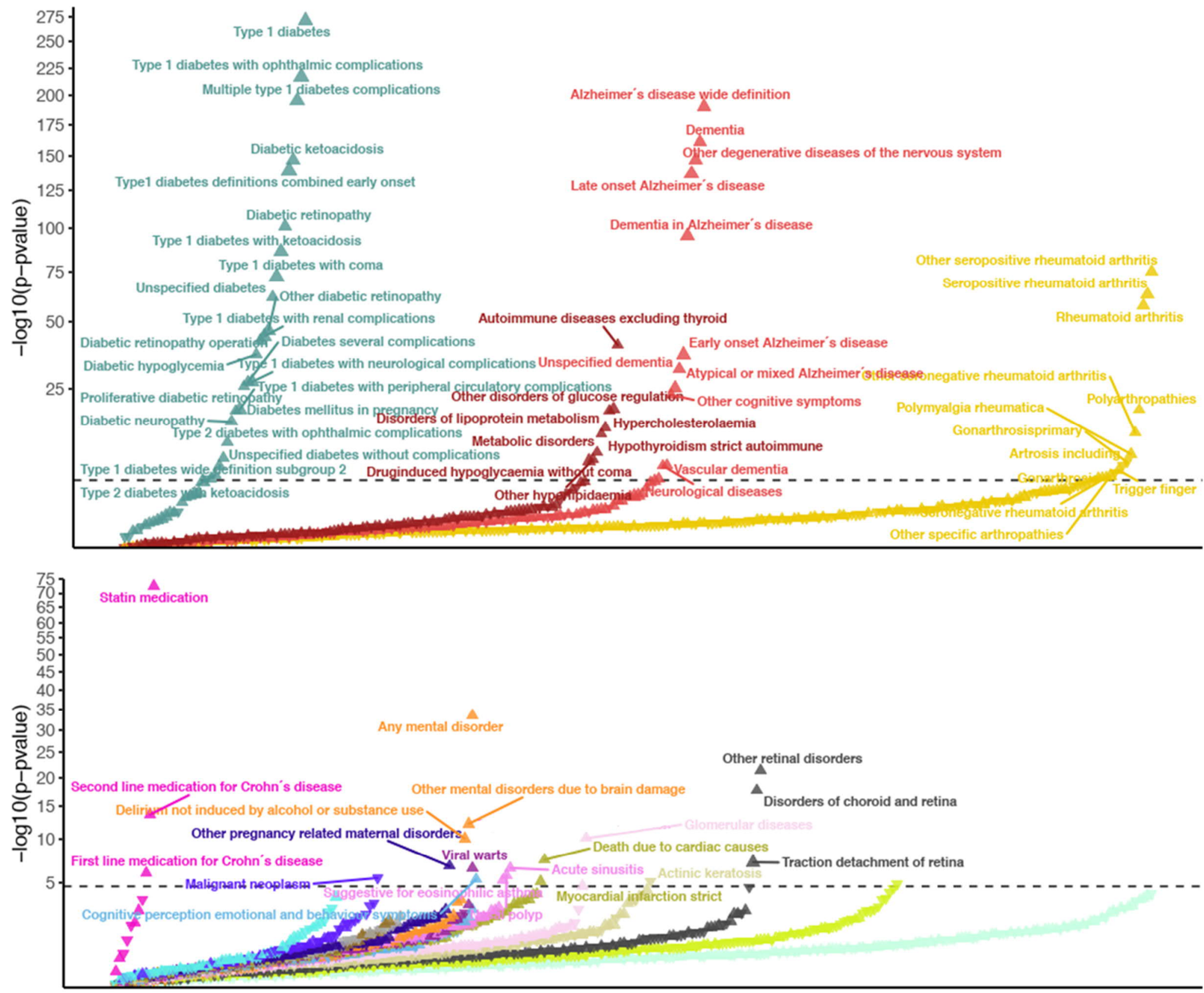

Outcomes
Cardiovascular diseases

Congenital malformations, deformations and chromosomal abnormalities

Diabetes and complications

Diseases of the blood and blood-forming organs

Diseases of the ear and mastoid process

- Diseases of the eye and adnexa

Diseases of the skin

Endocrine and metabolic diseases excluding diabetes Factors influencing health status and healthcare contacts

Gastrointestinal diseases

Genitourinary diseases

- Infections

Mental and behavioral disorders

- Musculoskeletal diseases

- Neoplasms

- Neurological diseases

Other symptoms, signs and abnormal clinical and laboratory findings

- Pregnancy, childbirth, and puerperium

Prescriptions

Odds ratio

v 0.8

- 1.2

$\Delta \quad 1.6$

Direction of effect

- High PRS $\rightarrow$ Higher risk

v High PRS $\rightarrow$ Lower risk 
medRxiv preprint doi: https://doi.org/10.1101/2022.02.17.22271136; this version posted February 18, 2022. The copyright holder for this preprint (which was not certified by peer review) is the author/funder, who has granted medRxiv a license to display the preprint in

It is made available under a CC-BY-NC-ND 4.0 International license

30

Figure 5A-E. Association between different HLA-allele subclasses (y-axis) and dementia causing diseases, autoimmune diseases and their complications (x-axis). The HLA-types with at least one directionally consistent association with autoimmune diseases are bolded. Left side of the panel describes dementias and right side autoimmune diseases and their complications. *, false discovery rate $\mathrm{p}<0.02 ; * *, \mathrm{p}<0.01$.

A)

HLA-A

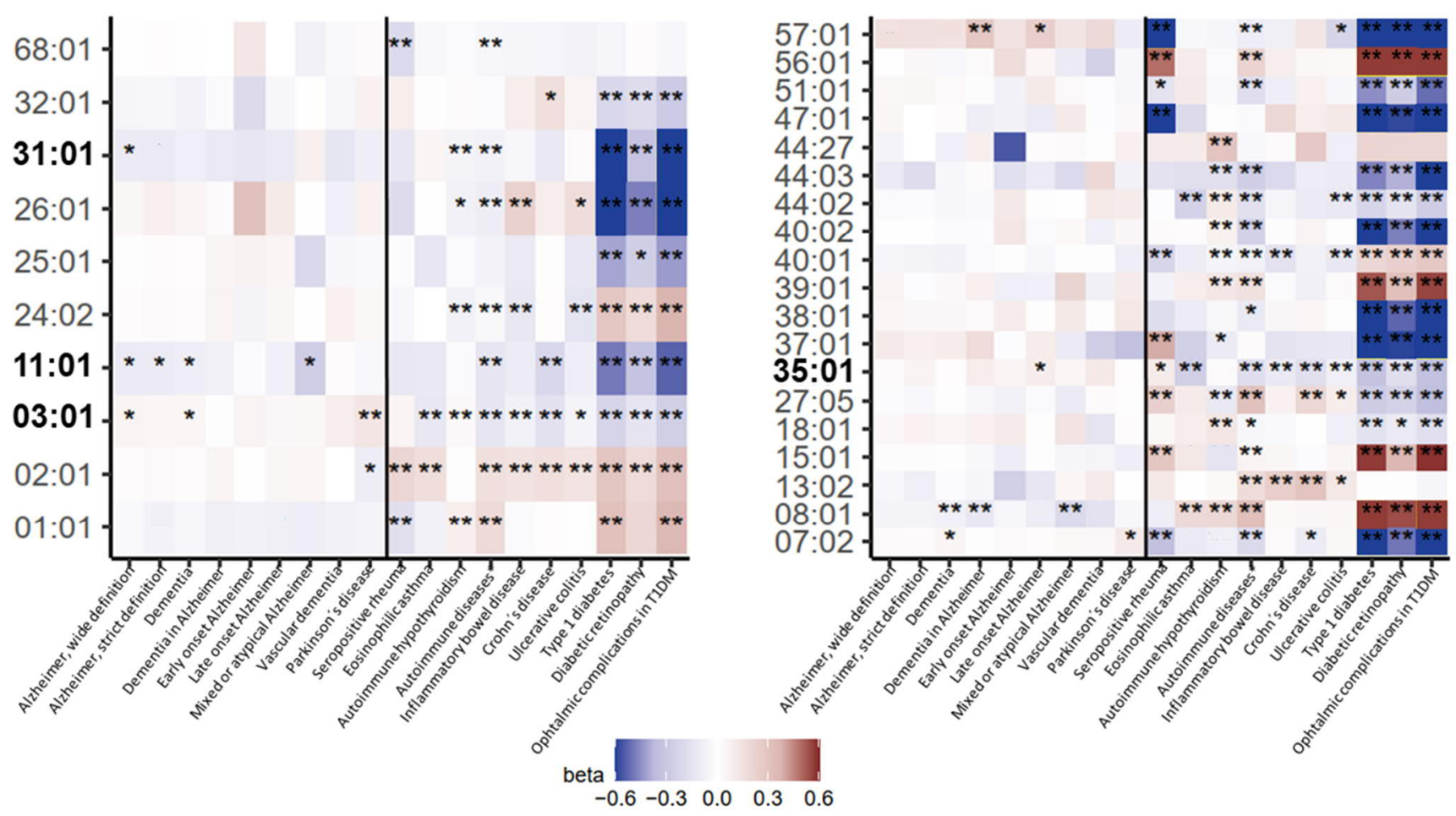

B)

HLA-C

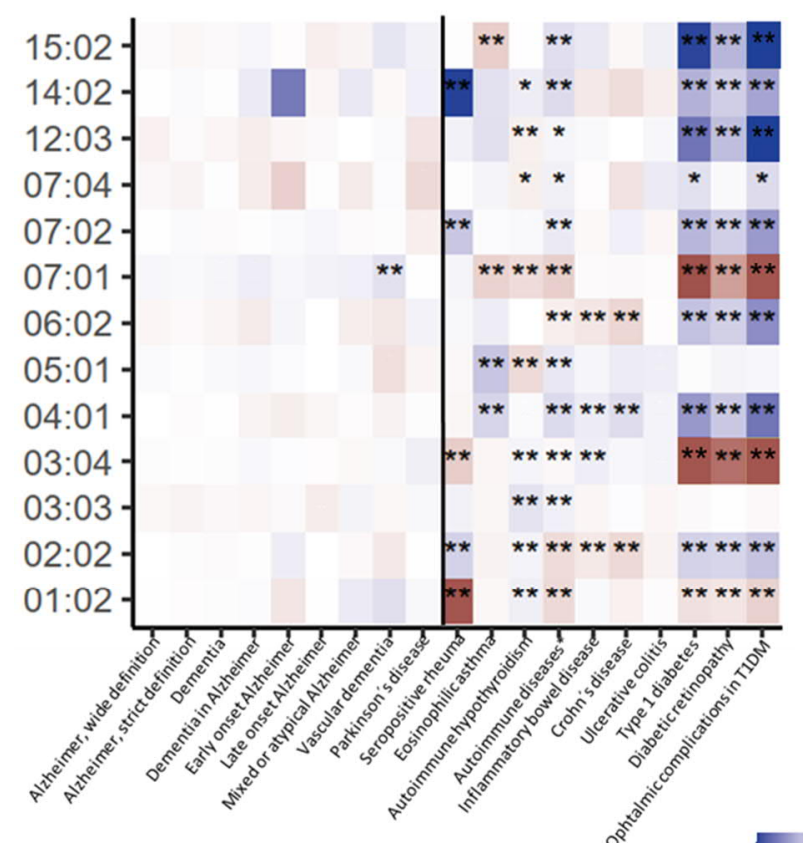

HLA-DQB1

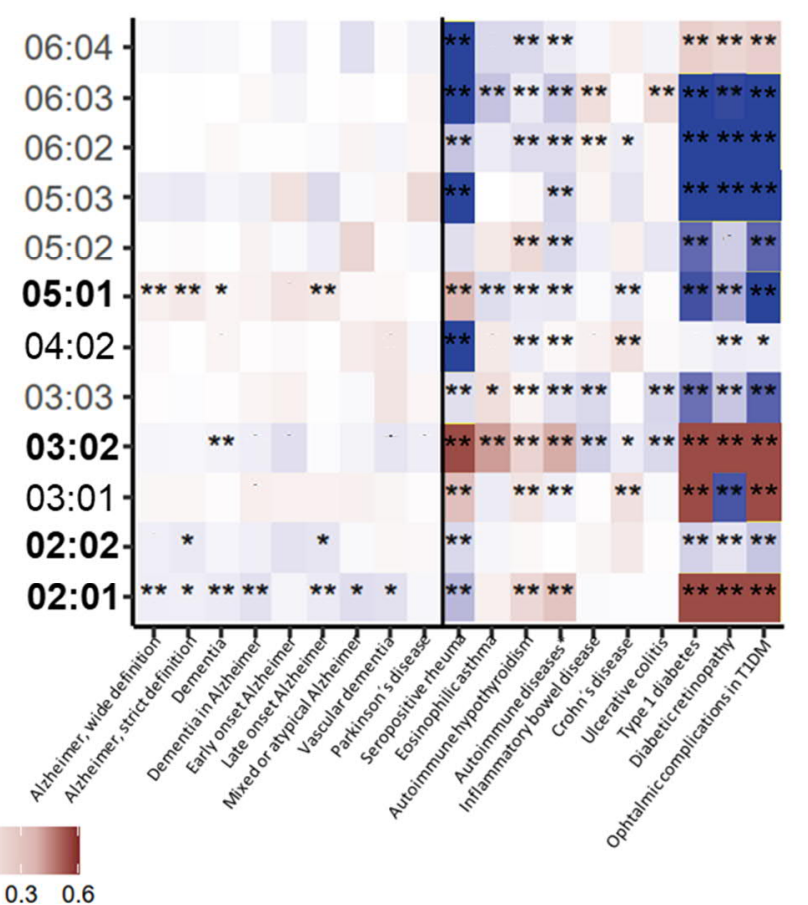


medRxiv preprint doi: https://doi.org/10.1101/2022.02.17.22271136; this version posted February 18, 2022. The copyright holder for this preprint (which was not certified by peer review) is the author/funder, who has granted medRxiv a license to display the preprint in It is made available under a CC-BY-NC-ND 4.0 International license .

\section{1}

C)

\section{HLA-DRB1}

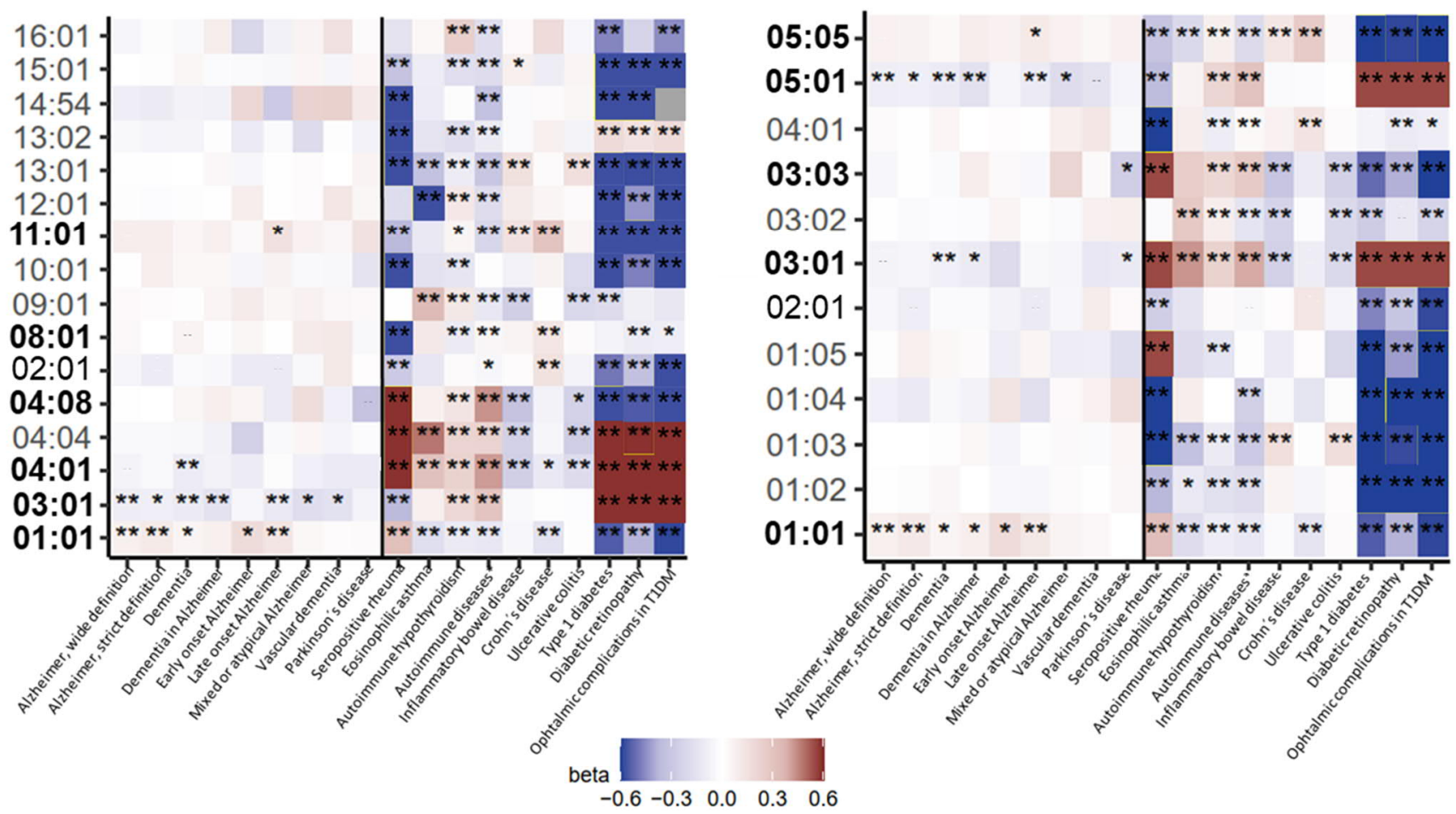

D)

\section{HLA-DPB1}

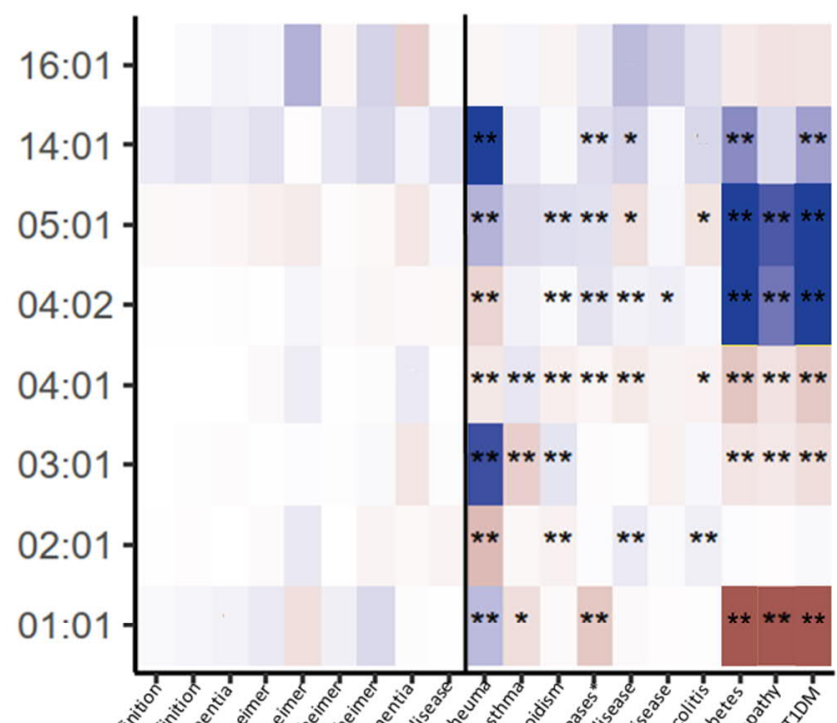

E)

\section{HLA-DRB3}

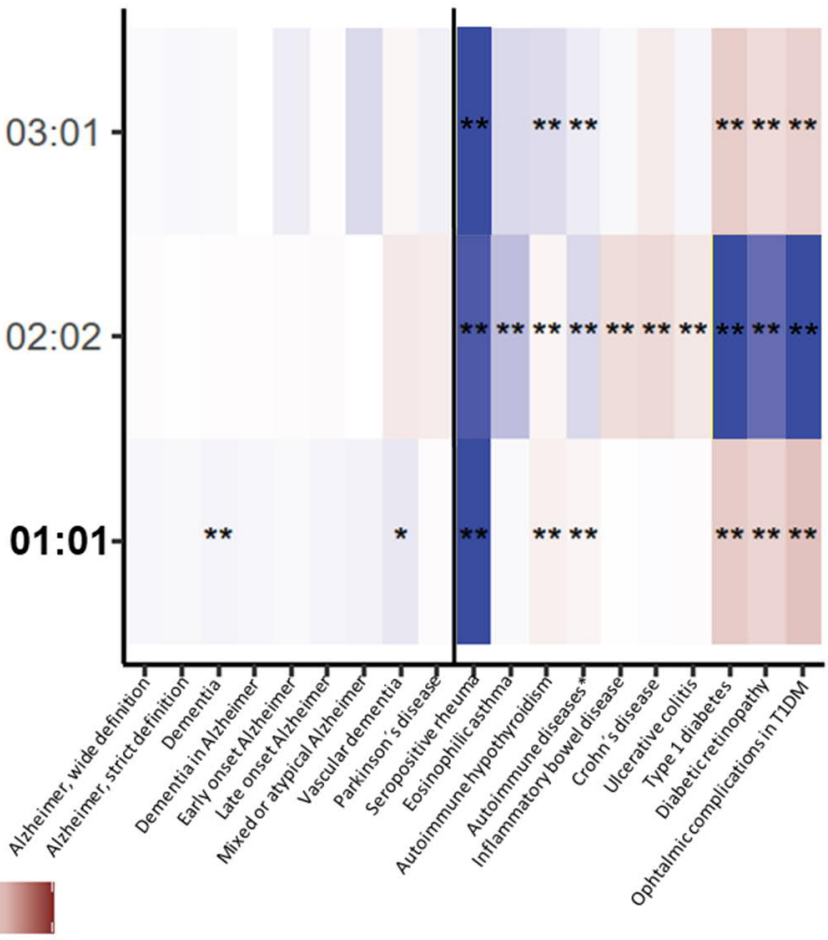


medRxiv preprint doi: https://doi.org/10.1101/2022.02.17.22271136; this version posted February 18, 2022. The copyright holder for this preprint (which was not certified by peer review) is the author/funder, who has granted medRxiv a license to display the preprint in

It is made available under a CC-BY-NC-ND 4.0 International license .

\section{2}

\section{HLA-DRB4}

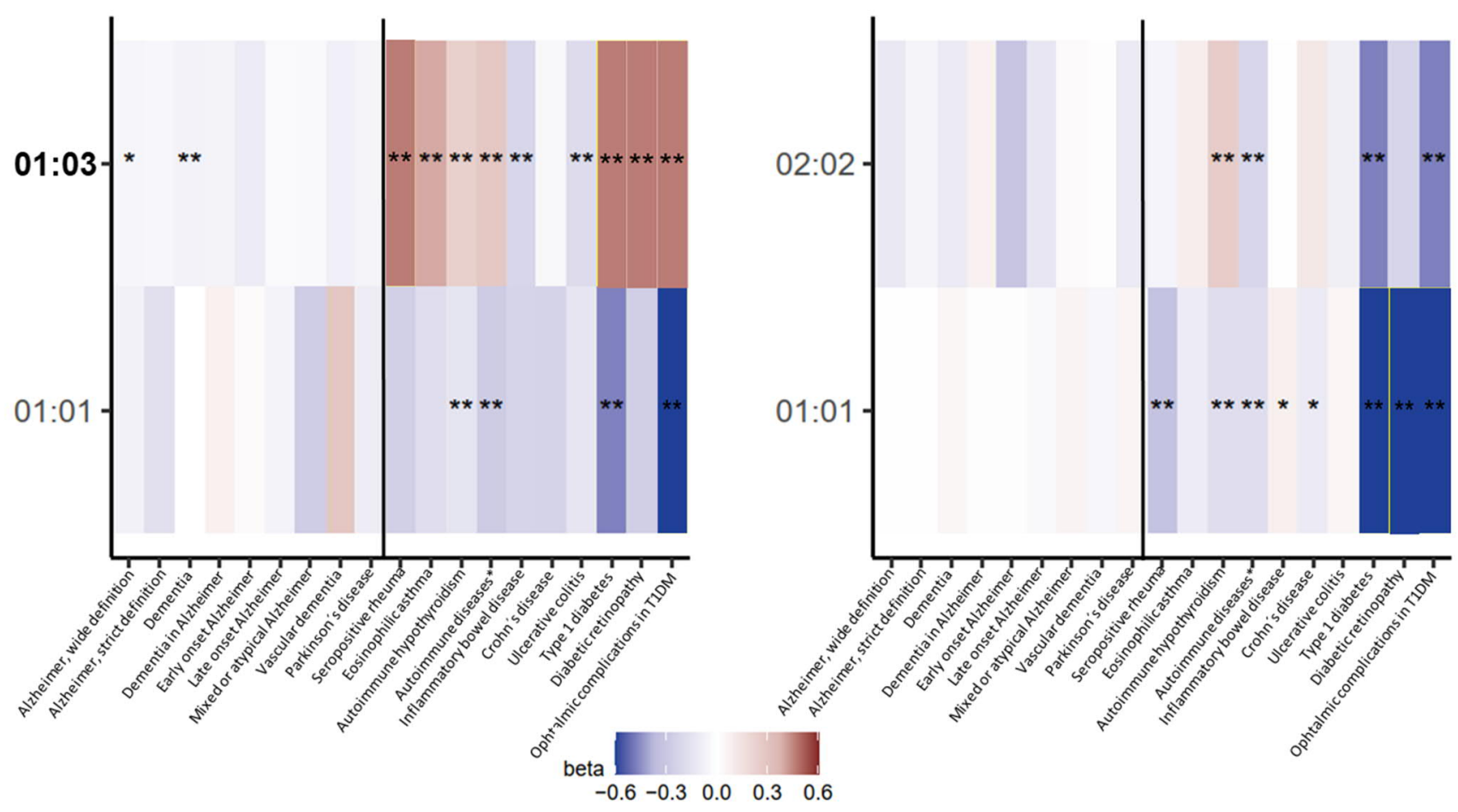

\section{HLA-DRB5}


medRxiv preprint doi: https://doi.org/10.1101/2022.02.17.22271136; this version posted February 18, 2022. The copyright holder for this preprint (which was not certified by peer review) is the author/funder, who has granted medRxiv a license to display the preprint in It is made available under a CC-BY-NC-ND 4.0 International license .

\section{3}

Table 1. Associations between dementias and anti-inflammatory medications from inverse probability weighted (IPW) analyses in the FinnGen study. The positive control IPW analyses validate the analysis protocol by replicating association between statin medication and coronary heart disease. The negative control IPW analyses validate the associations between each antiinflammatory medication and dementias by replicating the null finding between anti-inflammatory medications and coronary heart disease.

The baseline variables in IPW analyses were birth year, sex, ten principal components and timevarying variables statin, ACE-blocker, AT-blocker, renin-blocker, calcium channel blocker, any diuretic, insulin, metformin, other diabetes drug, depression medication, antipsychotic, and anticoagulant use as well as time varying any cancer, myocardial infarction, atrial fibrillation, heart failure, venous thromboembolism, ischemic stroke, intracerebral haemorrhage, subarachnoid haemorrhage, obesity, sleep apnoea, and chronic obstructive pulmonary diseases, with informative censoring included. Because of limited number of participants, TNF- $\alpha$ inhibitors users in dementias include also baseline users, other analyses include only medication-free individuals at baseline. The estimates for randomized control trials are from references 14 to 17 . Secondary prevention randomized control trial was used for methotrexate because no primary prevention trial was available. MR-PRS, Mendelian randomization based polygenic risk score for Alzheimer's disease; NSAID, non-steroidal anti-inflammatory drugs; TNF- $\alpha$, tumor necrosis factor $\alpha$; IPW, inverse probability weighted analyses; RCT, randomized control trials. 
medRxiv preprint doi: $h t$ tps://doi.org/10.1101/2022.02.17.22271136; this version posted February 18, 2022. The copyright holder for this preprint (which was not certified by peer review) is the author/funder, who has granted medRxiv a license to display the preprint in It is made available under a CC-BY-NC-ND 4.0 International license .

34

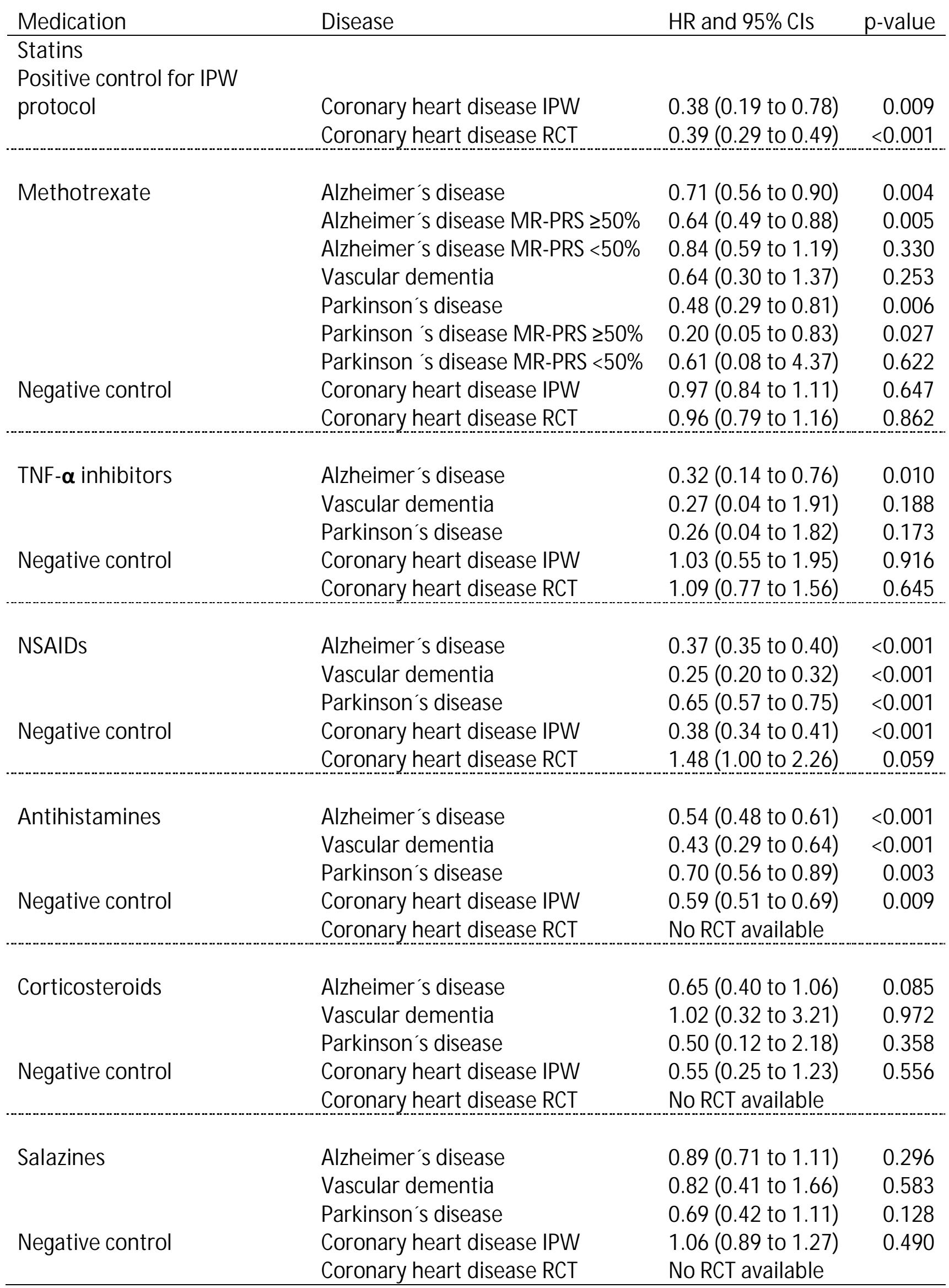


medRxiv preprint doi: https://doi.org/10.1101/2022.02.17.22271136; this version posted February 18, 2022. The copyright holder for this preprint (which was not certified by peer review) is the author/funder, who has granted medRxiv a license to display the preprint in It is made available under a CC-BY-NC-ND 4.0 International license .

35

Supplementary Table 1. Significant backward Mendelian randomization results with p-value below 0.05. These hazard ratios were not stronger than observed in forward Mendelian randomization analyses in Supplementary Figure 1A and B.

\begin{tabular}{lllll} 
Exposure & Outcome & Number of SNPs & HR (95\% Cls) & p-value \\
\hline Alzheimer's disease & FSC-A on plasmacystoid dendritic cell & 20 & $0.85(0.73$ to 0.98$)$ & 0.0275 \\
Late-onset Alzheimer's disease & Protein S100-A13 & 13 & $0.51(0.41$ to 0.63$)$ & $\varangle .001$
\end{tabular}


medRxiv preprint doi: https://doi.org/10.1101/2022.02.17.22271136; this version posted February 18, 2022. The copyright holder for this preprint (which was not certified by peer review) is the author/funder, who has granted medRxiv a license to display the preprint in It is made available under a CC-BY-NC-ND 4.0 International license .

36

Supplementary Figure 1A. Mendelian randomization sensitivity analyses for one standard deviation change in biomarker levels associated with late onset Alzheimer's diseases and with at least 3 SNPs available. The source of outcome and MR-base outcome identifier is described below the biomarker. Black $=$ inverse variance weighted, grey $=$ weighted median, blue $=$ simple mode, red $=$ Egger Mendelian randomization.

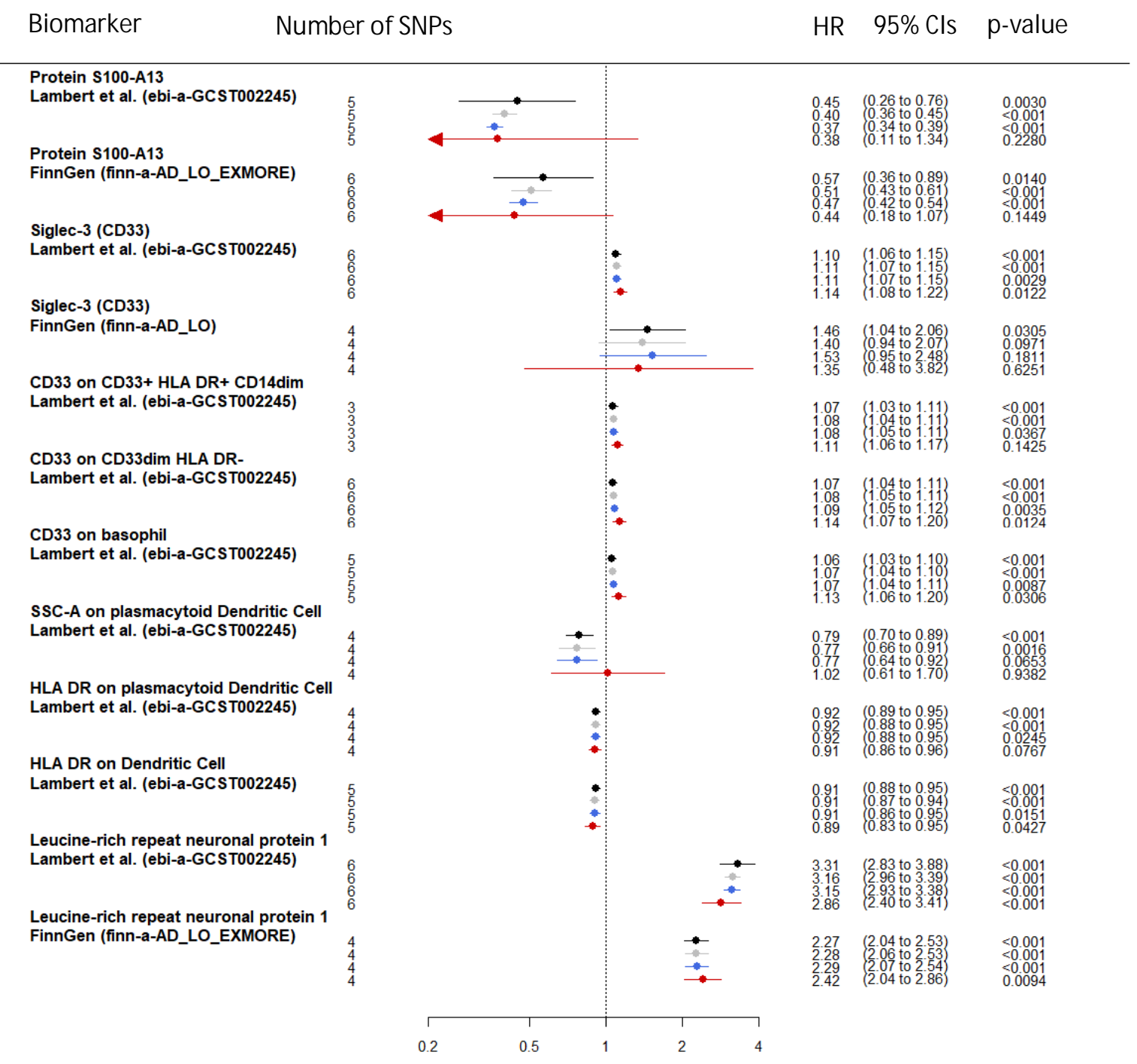


medRxiv preprint doi: https://doi.org/10.1101/2022.02.17.22271136; this version posted February 18, 2022. The copyright holder for this preprint (which was not certified by peer review) is the author/funder, who has granted medRxiv a license to display the preprint in

It is made available under a CC-BY-NC-ND 4.0 International license .

37

Supplementary Figure 1B. Mendelian randomization sensitivity analyses for one standard deviation change in biomarker levels associated with other dementias than late onset Alzheimer's diseases and with at least 3 SNPs available. The source of outcome and MR-base outcome identifier is described below the Biomarker. Black $=$ inverse variance weighted, grey $=$ weighted median, blue $=$ simple mode, red $=$ Egger Mendelian randomization .

Outcome

Alzheimer's disease

Atypical or mixed Alzheimer's disease

Early onset Alzheimer's disease

Dementia in Alzheimer disease

Dementia

Vascular dementia

Parkinson's disease

\section{Biomarker}

Monocyte percentage of white cells Lambert et al. (ieu-a-298)

Sum basophil neutrophil counts Lambert et al. (ieu-a-298)

FSC-A on plasmacytoid Dendritic Cell Kunkle et al. (ieu-b-2)

Monocyte percentage Lambert et al. (ieu-a-298)

Zinc-alpha-2-glycoprotein Lambert et al. (ieu-a-297)

Zinc-alpha-2-glycoprotein Kunkle et al. (ieu-b-2)

Leucine-rich repeat neuronal protein 1 FinnGen (finn-a-AD_AM_EXMORE)

Leucine-rich repeat neuronal protein 1 FinnGen (finn-a-AD_EO_EXMORE)

Leucine-rich repeat neuronal protein 1 FinnGen (finn-a-F5_ALZHDEMENT)

Leucine-rich repeat neuronal protein 1 FinnGen (finn-a-F5_DEMENTIA)

Leucine-rich repeat neuronal protein 1 FinnGen (finn-a-F5_VASCDEM)

CX3CR1 on monocyte Nalls et al. (ieu-b-7)

CX3CR1 on CD14+ CD16+ monocyte Nalls et al. (ieu-b-7)

CX3CR1 on CD14+ CD16- monocyte Nalls et al. (ieu-b-7)

Transmembrane glycoprotein NMB Nalls et al. (ieu-b-7)
Number of SNPs

HR $95 \%$ Cls p-value

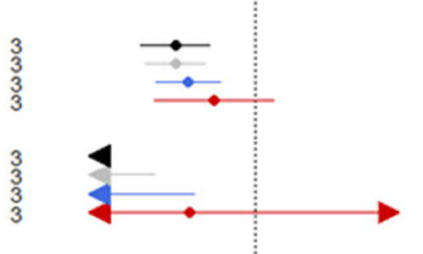

0.46
0.46
0.52
$0.67 \quad(0.33$ to 0.64$)$
0.38 to 0.61$)$

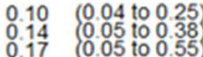

$\begin{array}{lll}0.17 & 0.05 \text { to } 0.55) & 0.001\end{array}$

$0.75 \quad(0.65$ to 0.86$) \quad<001$

3
3
3
3

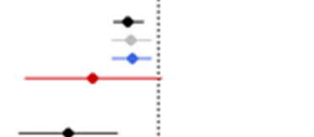

0.75
0.77
0.53

0.64 to 0.92

$(0.64$ to 0.93

$\begin{array}{llll}0.42 \quad(0.26 & \text { to } & 0.67) \\ 0.43 & 0.32 & 0.50 & 0.58)\end{array}$

3
3
3
3

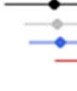

$-$

4
4
4
4

4
4
4

4
4
4
4

4
4
4
4

4
4
4
4

4
4
4
4

4
4
4
4

3
3
3
3

3
3
3
3

3
3
3
3

3
3
3
3
4
4
4
4

$\left.\begin{array}{l}1.08 \\ 1.10 \\ 1.0210 \\ 0.96 \text { to } 1.129\end{array}\right)$

0.0907

$\begin{array}{ll}1.98 & (1.55 \text { to } 2.52 \\ 2.01 & (1.699102 .40 \\ 2.03 & 1.71 \text { to } 2.42 \\ 2.09 & 1.35 \text { to } 3.23)\end{array}$

$<0.001$

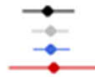

0.0802

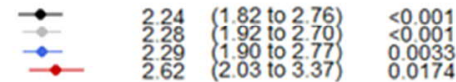

$\begin{array}{lll}2.04 & (1.85 \text { to } 2.25) & <0.001 \\ 2.05 & (1.85 \text { to } 2.27 & <0.001\end{array}$

$\left.\begin{array}{lll}2.08 & (1.88 \text { to } 2.30 \\ 2.20 & (1.91 \text { to } 2.54\end{array}\right) \quad 00.001$

$1.70 \quad(1.52$ to 1.91$) \quad<0.001$

$\begin{array}{lll}1.73 & 1.61 \text { to 1.85) } & <0.001 \\ 1.86 & (1.63 \text { to 2.13) } & 0.0120\end{array}$

$1.53 \quad(1.33$ to 1.77$) \quad<0.001$

$\begin{array}{lll}1.56 & \left(\begin{array}{ll}1.35 \\ 1.701 .82\end{array}\right) & 0.0091 \\ 1.38 \text { to 2.11) } & 0.0382\end{array}$

$\stackrel{\circ}{\circ}$

0.85
0.84
0.84

\begin{tabular}{l}
0.79 to 0.91$)$ \\
0.79 \\
0.00090 \\
$(070500$ \\
\hline
\end{tabular}

$<0.001$
$<0.001$

0.0571
0.4680

$0.85 \quad 0.80$ to 091

$(0.80$ to 0.92

$<0.001$

$0.91(0.77$ to 1.07$)$

0.0512

$0.86 \quad(0.81$ to 0.91$) \quad<0.001$

$<0.001$
0.0557
0.4545

$\begin{array}{lll}1.25 & (1.03101 .52) & 0.0271 \\ 1.25 & 1.11 \text { to } 1.40) & <0.001\end{array}$

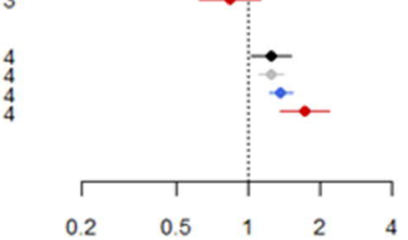


medRxiv preprint doi: $h$ ttps://doi.org/10.1101/2022.02.17.22271136; this version posted February 18, 2022. The copyright holder for this preprint (which was not certified by peer review) is the author/funder, who has granted medRxiv a license to display the preprint in It is made available under a CC-BY-NC-ND 4.0 International license .

38

Supplementary Figure 2. Phenome-wide association analyses for the 126 biomarkers, effect size describes one standard deviation change in biomarker levels. All biomarker and outcomes pairs that passed false discovery rate correction of 5\% are presented. Most biomarkers associated with only few outcomes and the grey boxes indicate no association after false discovery rate correction of 5\% or lack of common SNPs.

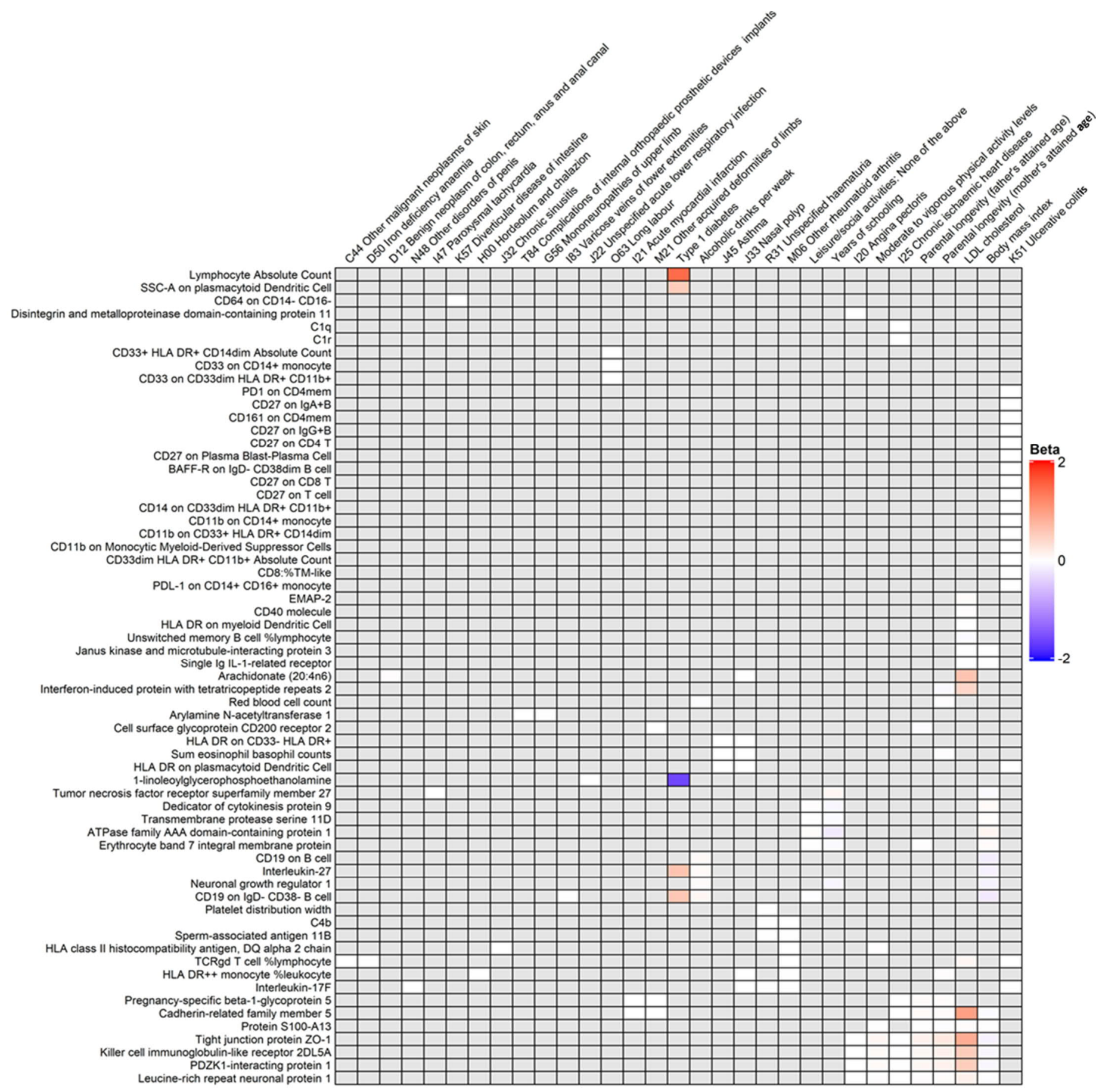


medRxiv preprint doi: https://doi.org/10.1101/2022.02.17.22271136; this version posted February 18, 2022. The copyright holder for this preprint (which was not certified by peer review) is the author/funder, who has granted medRxiv a license to display the preprint in

It is made available under a CC-BY-NC-ND 4.0 International license .

\section{9}

Supplementary Figure 3. Plasma protein associations with dementia for one standard deviation change in protein levels from the Whitehall II cohort. The analyses included 8 proteins that had SNPs clustered around $A P O E$ and associated with at least three dementia subtypes in Mendelian randomization analyses. Analyses are first adjusted for age and sex and then additionally for APOE status.

\section{Protein measured from plasma}

Endothelial monocyte-activating polypeptide 2

Endothelial monocyte-activating polypeptide 2 APOE adjusted

Interferon-induced protein with tetratricopeptide repeats 2

Interferon-induced protein with tetratricopeptide repeats 2 APOE adjusted

Killer cell immunoglobulin-like receptor 2DL5A

Killer cell immunoglobulin-like receptor 2DL5A

Killer cell immunoglobulin-like receptor 2DL5A APOE adjusted

Killer cell immunoglobulin-like receptor 2DL5A APOE adjusted

Leucine-rich repeat neuronal protein 1:Cytoplasmic domain

Leucine-rich repeat neuronal protein 1:Cytoplasmic domain APOE adjusted

Leucine-rich repeat neuronal protein 1:Extracellular domain

Leucine-rich repeat neuronal protein 1:Extracellular domain APOE adjusted

Tight junction protein ZO-1

Tight junction protein ZO-1 APOE adjusted

Tumor necrosis factor receptor superfamily member $13 \mathrm{C}$

Tumor necrosis factor receptor superfamily member $13 \mathrm{C}$ APOE adjusted

Valine--tRNA ligase

Valine--tRNA ligase APOE adjusted

\section{HR $\quad 95 \%$ Cls p-value}

$1.02 \quad$ (0.91 to 1.14) 0.750

$1.11 \quad(0.97$ to 1.27$) \quad 0.140$

$0.79 \quad(0.70$ to 0.88$) \quad<0.001$

$0.84 \quad$ (0.73 to 0.97$) \quad 0.010$

$1.03 \quad(0.90$ to 1.18$) \quad 0.640$

$0.95 \quad$ (0.85 to 1.07$) \quad 0.390$

$0.98 \quad$ (0.83 to 1.17$) \quad 0.860$

$0.95 \quad$ (0.83 to 1.09 ) $\quad 0.480$

$1.38 \quad(1.23$ to 1.54$) \quad<0.001$

$1.01 \quad(0.83$ to 1.22$) \quad 0.930$

$1.08 \quad(0.95$ to 1.24$) \quad 0.250$

$1.08 \quad$ (0.91 to 1.28$) \quad 0.390$

$0.96 \quad(0.86$ to 1.08$) \quad 0.520$

$0.95 \quad$ (0.83 to 1.09$) \quad 0.460$

$1.00 \quad$ (0.88 to 1.14$) \quad 0.950$

$1.01 \quad$ (0.87 to 1.18$) \quad 0.870$

$0.99 \quad(0.89$ to 1.11$) \quad 0.930$

$0.95 \quad$ (0.84 to 1.09$) \quad 0.490$ 
medRxiv preprint doi: https://doi.org/10.1101/2022.02.17.22271136; this version posted February 18, 2022. The copyright holder for this preprint (which was not certified by peer review) is the author/funder, who has granted medRxiv a license to display the preprint in It is made available under a CC-BY-NC-ND 4.0 International license

40

Supplementary Figures 4 A-H. KEGG functional pathway enrichment analyses for all proteins and receptors that associated with dementias in Mendelian randomization analyses. The estimated causal betas were used in place of log fold change. 
A)

\section{CYTOKINE-CYTOKINE RECEPTOR INTERACTION}

Chemokines
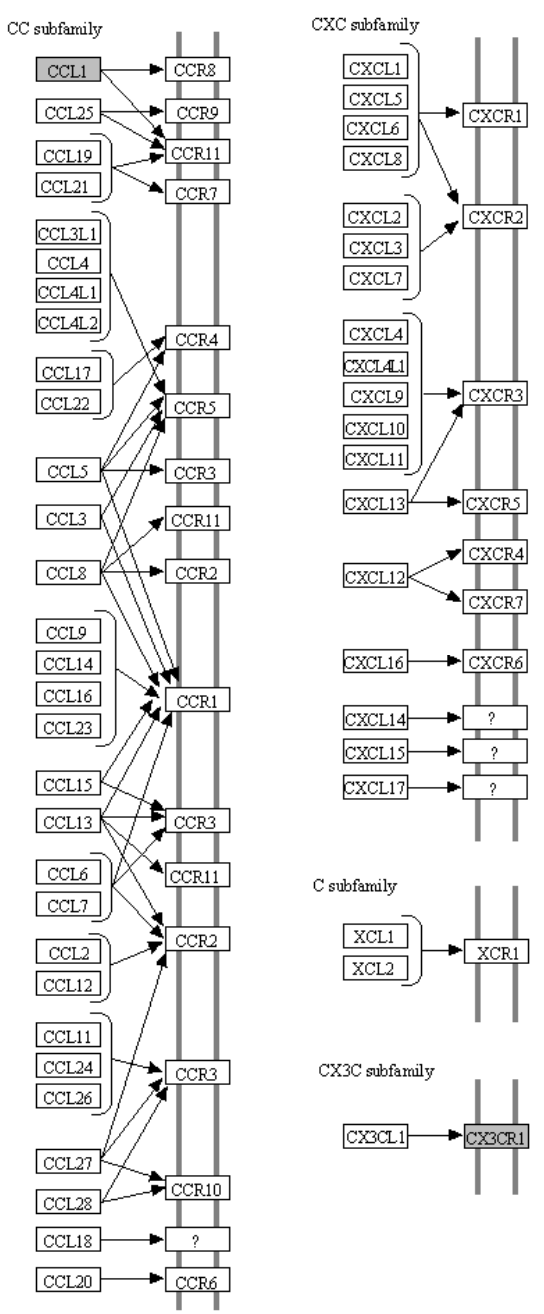

CX3C subfamily

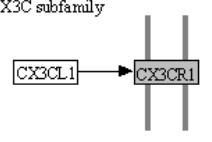

The class I helic al cytolines

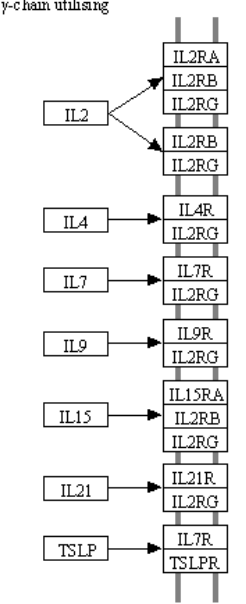

IL4like

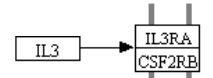

ILS $\longrightarrow$ IISSRA

CSF2 $\longrightarrow$ CSF2RA

IL4 $\longrightarrow$ IILAR

IL13

$\frac{11}{113 R \mathrm{~A} A 1}$

Prolac in farmily

GH1

PRL $\longrightarrow$ PRLR

$\longrightarrow$ TPO $\longrightarrow$ MPL

CSF3 C CSF3R

$\longrightarrow$ LEP $\longrightarrow$ LEPR

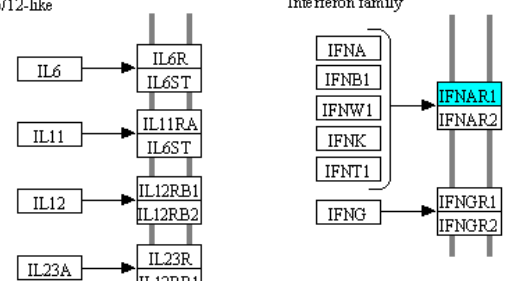

IL23A $\longrightarrow$ IIL23R

$\longrightarrow$ IIL27 $\longrightarrow$ IL27RA

$\underset{\mathrm{IL} 31}{\longrightarrow} \stackrel{\mathrm{II31RA}}{\mathrm{LOSMR}}$

$\longrightarrow$ IL35 $\longrightarrow$ LL12RE2

\begin{tabular}{ll}
\hline CLCF1 \\
\hline CNTF
\end{tabular}$\rightarrow$\begin{tabular}{|l} 
CNTFR \\
\hline LIFR \\
\hline ILGST \\
\hline I
\end{tabular}

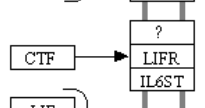

$\underset{\mathrm{LIF}}{\mathrm{LIFR}}$

\begin{tabular}{c} 
human \\
\hline OSM \\
\hline
\end{tabular}

$\stackrel{\text { human imurine }}{\longrightarrow \text { OSMR }}$

\section{The class II helizal cytokines}

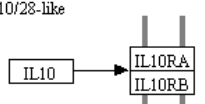

$\longrightarrow$ IL10RP

IL19.

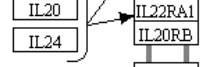

$\longrightarrow$ IL22 $\longrightarrow$ IL22RA1

$\longrightarrow$ IL26 $\longrightarrow$ IIL20RA

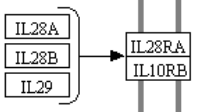

\section{ILI-Hike cytolines}

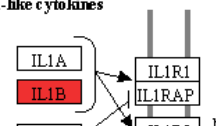

IILRN $\rightarrow$ IILIR2 No signal

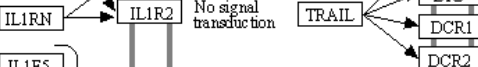

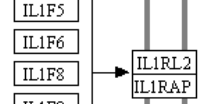

IL1F9

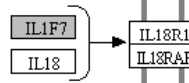

$\stackrel{\text { ST2 }}{\longrightarrow \text { IL1RAP }}$

IL17-like cytolines

\begin{tabular}{|l|}
\hline IL17A \\
\hline $\mathrm{IL17F}$
\end{tabular}$\rightarrow \underset{\mathrm{IL17RA}}{\mathrm{IL17RC}}$

$\longrightarrow$

$\longrightarrow$ IL17C $\longrightarrow$ IIITRE

IL17D $\longrightarrow$ ?

$\longrightarrow$ IL172 $\longrightarrow \frac{11717 \mathrm{RA}}{\mathrm{IIL17RB}}$

None hassified

ILL16 $\longrightarrow$ CD4

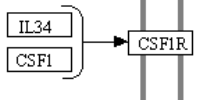

TNF Family

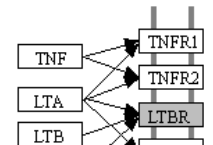

LTB

$$
\begin{array}{|l|}
\hline \mathrm{DCR} 3 \\
\hline 11
\end{array}
$$

4

FASLG $\rightarrow$ FAS

$\mathrm{VEGI} \longrightarrow \mathrm{DR3}$

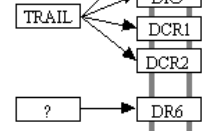

$\underset{\mathrm{EDDA}}{\mathrm{EDA}-\mathrm{A}} \longrightarrow$

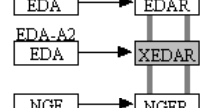

$\longrightarrow$ NGF $\longrightarrow$ NGFR

OPG

$\longrightarrow$

$\mathrm{CD} 70 \longrightarrow \mathrm{CD} 27$

$\mathrm{CD302} \longrightarrow \mathrm{CD} 30$

$\mathrm{CDAOLG} \longrightarrow \mathrm{CD} 40$

$41 \mathrm{BBL} \longrightarrow 41 \mathrm{BB}$

OX-4.2L $\longrightarrow 0 \times 40$

GITRL $\longrightarrow$ GITR

A.PIIL

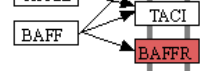

$? \longrightarrow \frac{1}{\mathrm{TROY}}$

$\longrightarrow \longrightarrow$ RELT
TGF $\beta$ family $\quad-$

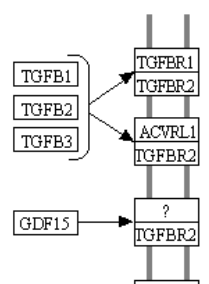

BMP10

INHA

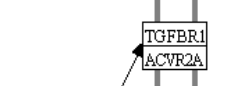

GDF10

GDF11

MSTN 1

INHBA

\begin{tabular}{|l|l|}
\hline INHBE \\
\hline \hline GDF1 \\
\hline ACTR2B \\
\hline GES
\end{tabular}

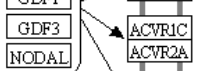

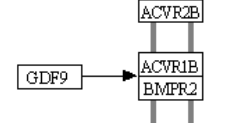

INHBC $\longrightarrow$ ?

INHBE $\longrightarrow$ ?

AMH

$\mathrm{MH}$

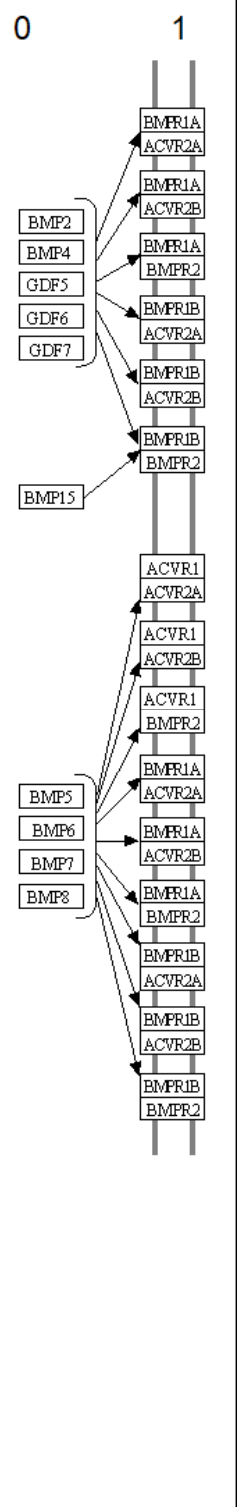


B)

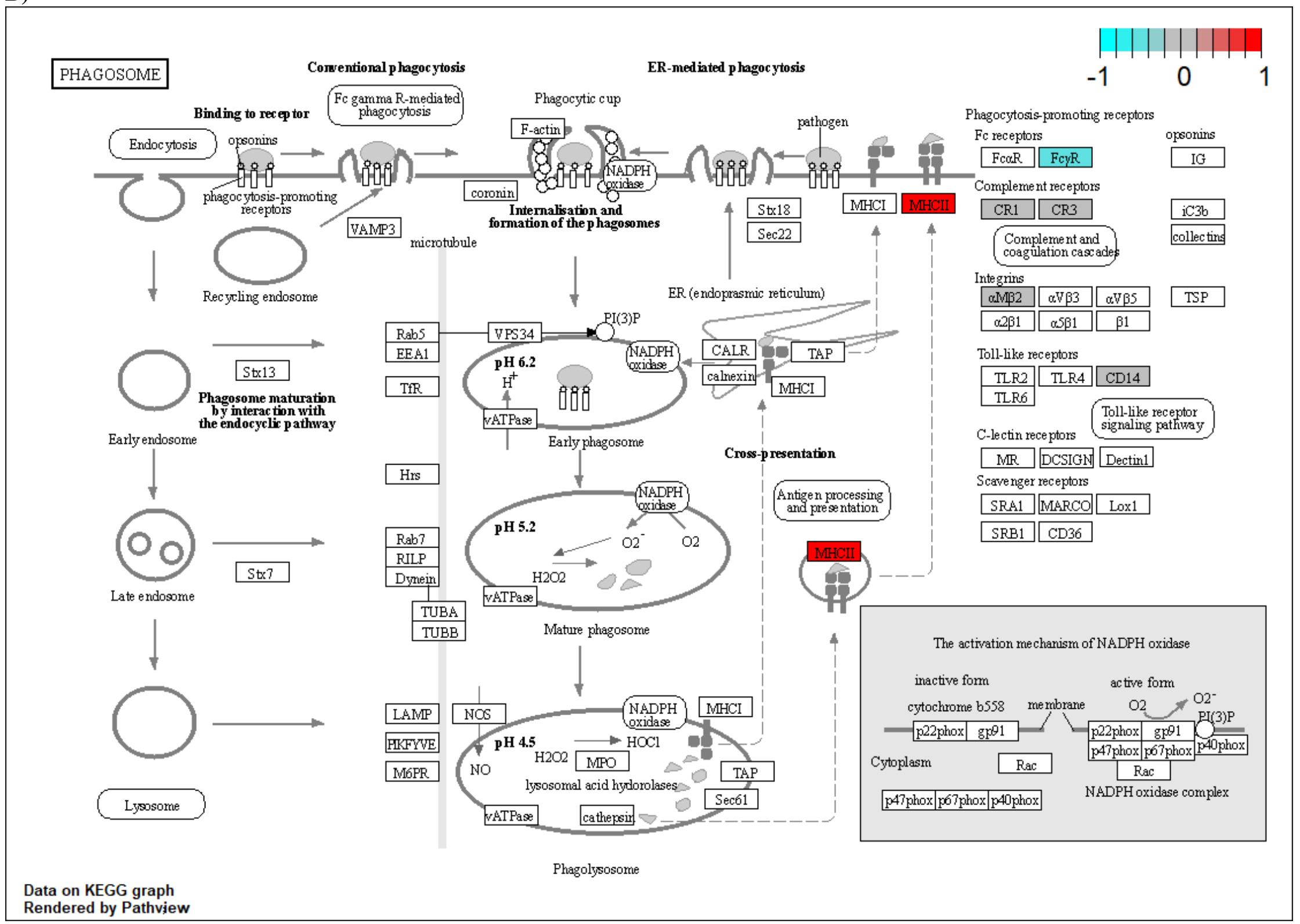


C)

\section{CELL ADHESION MOLECULES}

IMMUNE SYSTEM

$\longrightarrow \mathrm{CD} 2$

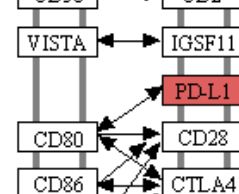

CD86 14 CTLA

ICOSLG ICOS

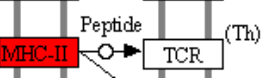

Peptide

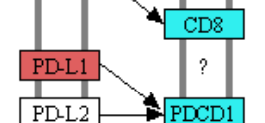

B?H3

B? 4

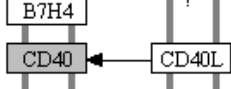

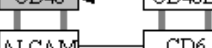

$P$ PVR $\longrightarrow$ CD226

TIVIT Th1)

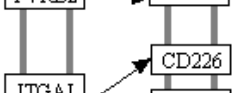

ITGAL $\longrightarrow$ CAM23

$\underset{\text { ITAM 23 }}{ } \longrightarrow \frac{\text { ITGAL }}{\text { ITGB2 }}$

CD22 PTPRC

(M)

$\mathrm{STH}_{\mathrm{SH}}$

$\mathrm{T}$ cell receptor
signaling pathway

Data on KEGG graph

dered by Pathview

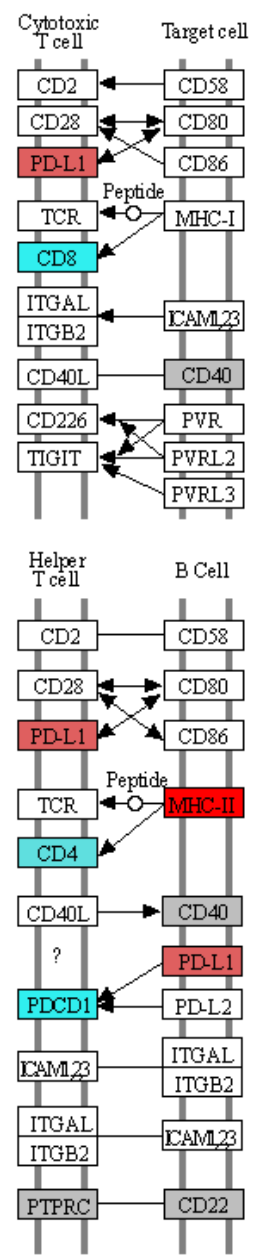

Endothelial cells CLDN OCLN OCLN ITM1 I I 1121 JAM2 2 JAM2 JAM3 ESAM ESAM $\mathrm{CDH}-\mathrm{CDH}$ PECAM PFCAMI \begin{tabular}{|lll}
\hline CD99 & \\
\hline &
\end{tabular}
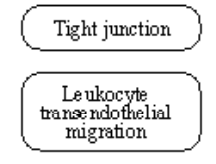

Le ukocyte Platelet $\mid$ ITGB2 2 SELPLG SELP

CD40 $C D 40 \mathrm{~L}$ ITGAL

ITGB2 I I

Platelet Endothelial

II

CD40L CD40

FECAM PECAMI

Complement and
coagulation cascade

\section{NEURAL SYSTEM}

Leukocyte Endothelial

Neuron
(mesynaptic) (postsynaptic)

PVRL1 PVRL3

PVRL3 PVRL1

$\mathrm{CDH} 2 \longrightarrow \mathrm{CDH} 2$

$\frac{1}{\mathrm{NCAM}}$

L1CAM LICAM

IGSF4 IGSF4

NEGR1 N

$1 \frac{1}{\text { NTNG1 }}$

NTNG2

PTPRF

\begin{tabular}{|l|l|}
\hline$S D C$ & SDC \\
$?$ & 1 \\
\hline ITGAN \\
\hline
\end{tabular}

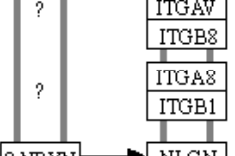

$\underset{\mathrm{N}}{\mathrm{B-NRXNA}} \longrightarrow$

Neuron
(growth cone)

$\frac{1}{\text { IGSF4B }}$

NRCAM - CNTN1

PTPRM PTPRM

$\underset{\mathrm{NCAM}}{\longrightarrow} \longrightarrow \mathrm{NCAM}$

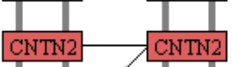

1121

$\longrightarrow \frac{\mathrm{CDH}}{\mathrm{CDH} 2}$
11111111

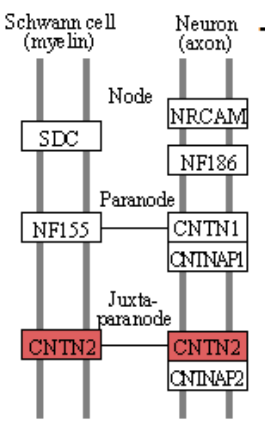

Epholial cells 1

$\frac{1}{\mathrm{CLDN}}$

OCLN OCLN

JAM1 JAM1

PURL1 $\longleftrightarrow$ PURL1

$\mathrm{CDHE} \leadsto \mathrm{CDHE}$

IGSF4 IGSF4

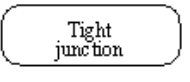

Schwarn cell(myelin)

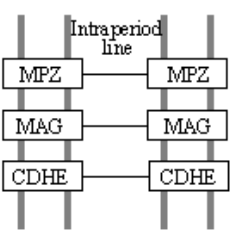

Addherens
junction

Spermatid Sertolicell

$\frac{\mathrm{CDHE}}{\mathrm{CDHE}}$

$\mathrm{CDH} 2-\mathrm{CDH} 2$

PVRL3 PVRL2

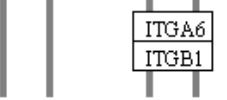

Ciliarybody

Pigment
Non-pigment
epithelia

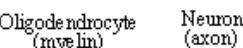

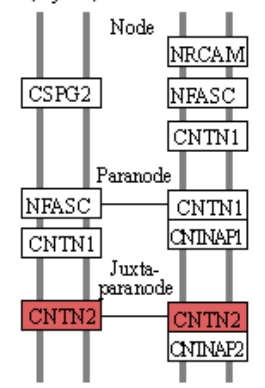

PVRL1 - IVRL3

PVRL3 PVRL1

$\mathrm{CDH} 3-\mathrm{CDH} 3$$$
\text { Myoblasts }
$$

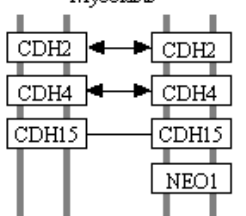

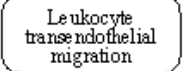


D)

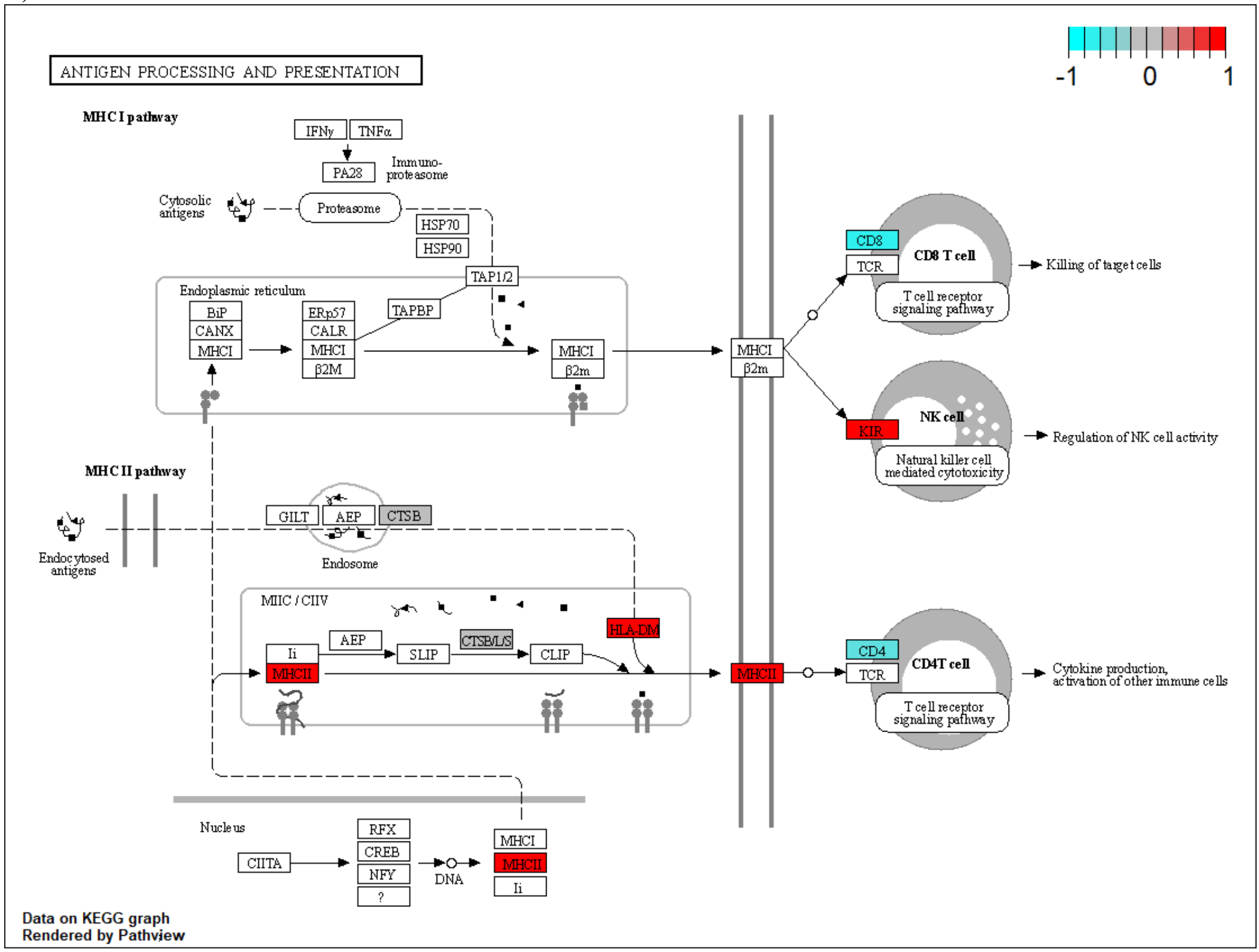


E)

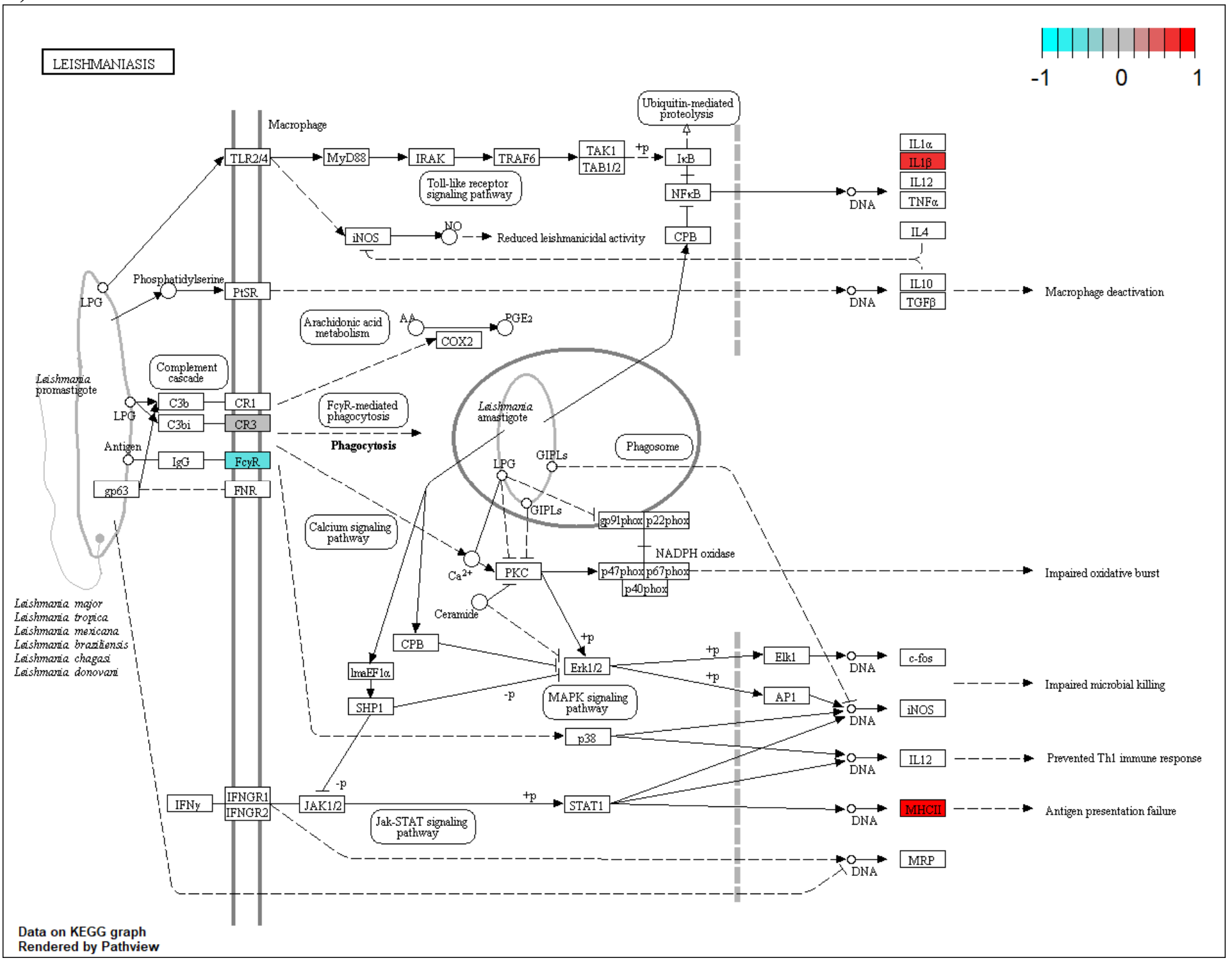


F)

SYSTEMIC LUPUS ERYTHEMATOSUS

|||||||||| $\mid$
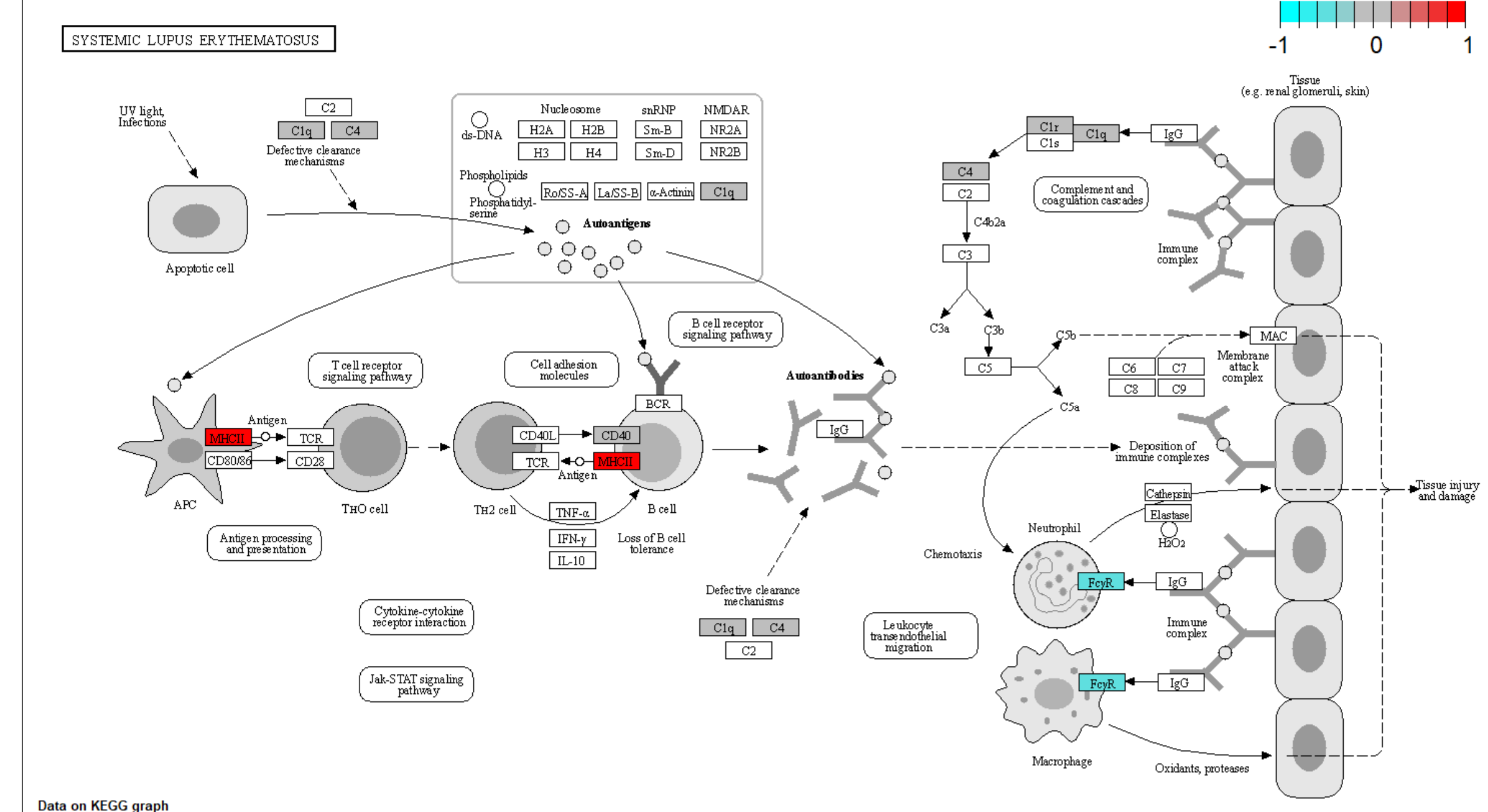

Data on KEGG graph 
G)

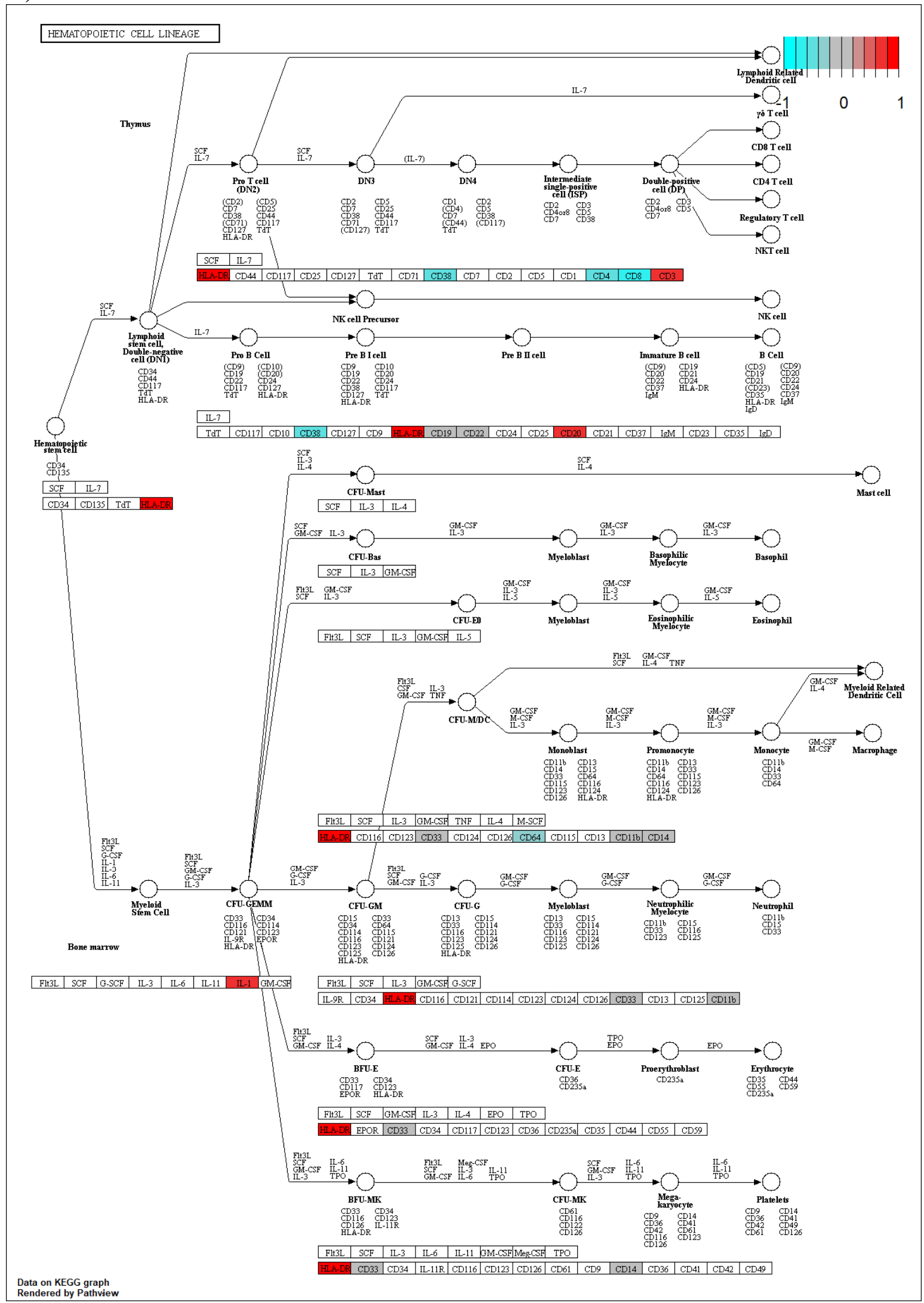


H)

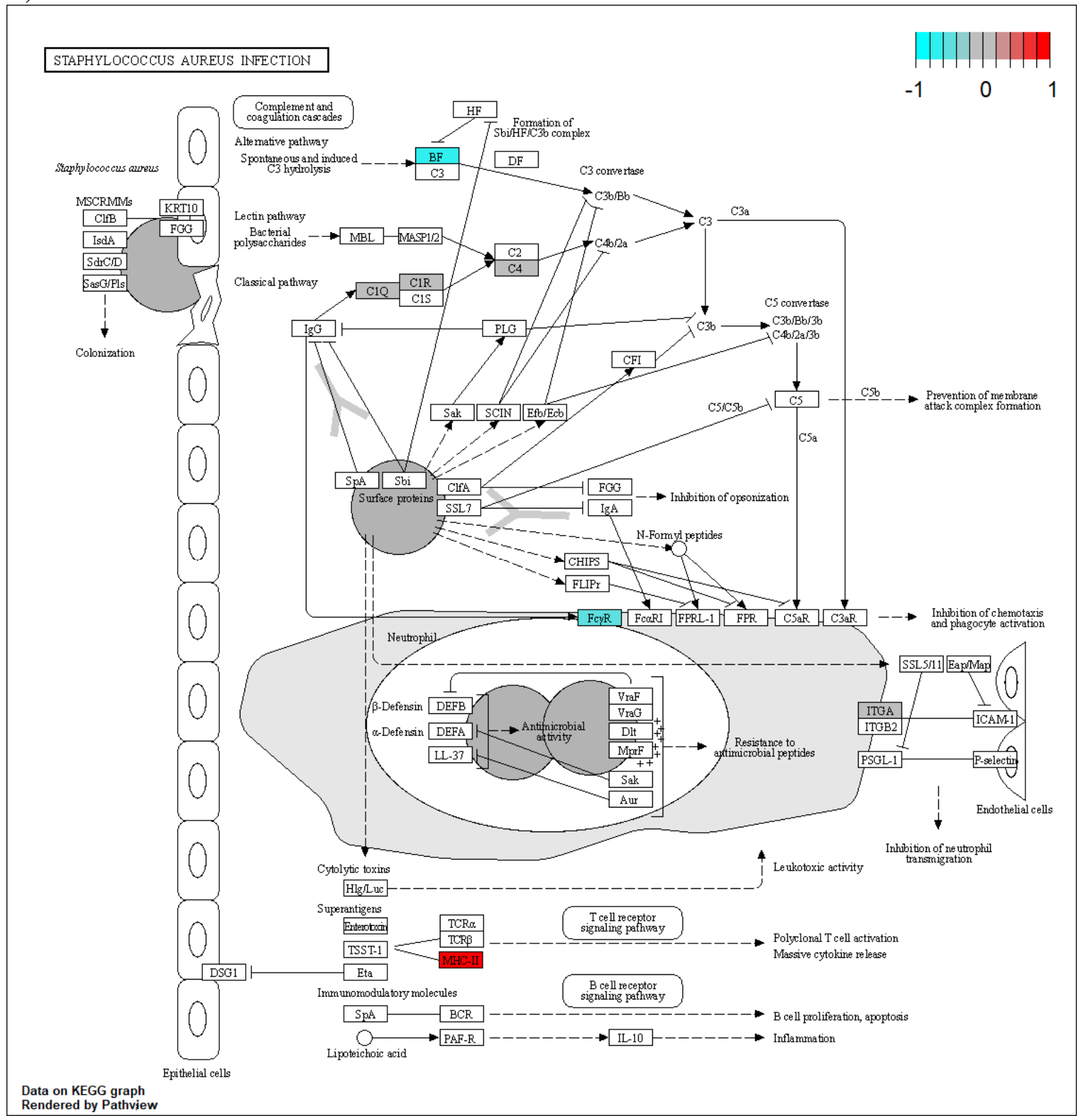


Supplementary Figure 5. ClueGO enrichment analyses for all the 76 proteins and receptors in the biomarkers that had evidence of causal association in Mendelian randomization analyses. All proteins that associated with Uniprot search terms "immune system" and "blood brain barrier" were used as a background dataset. GO-terms in blue were enriched in cluster where most significant associations were observed for cell adhesion molecules. In the red cluster, the most significant GO-term associations were observed for hematopoietic cell lineage. Big dots describe the GO-terms and small dots the genes associated with these terms.

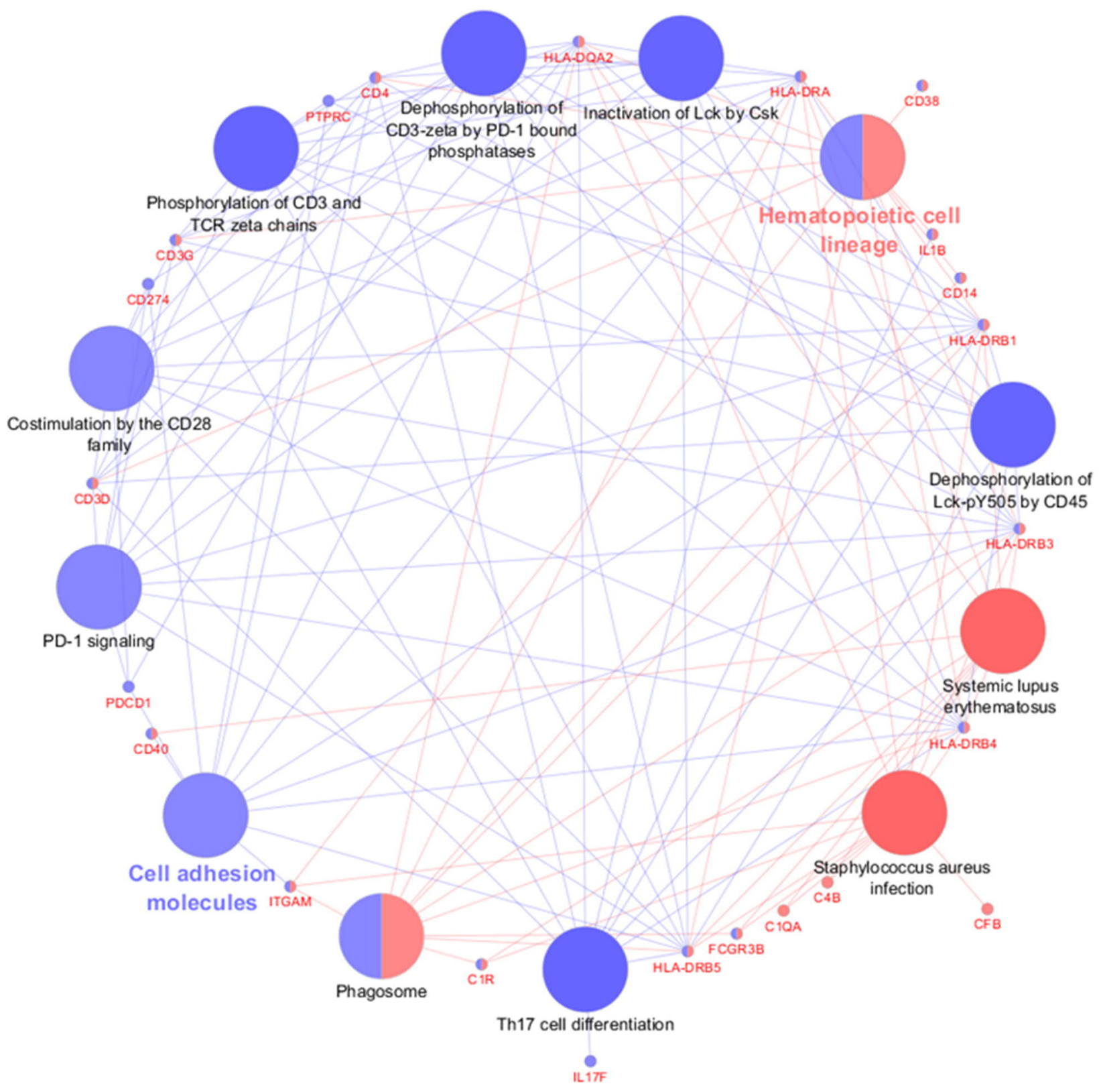


Supplementary Figures 6A-R. ConsensusPathDB shortest interaction path analyses for the 42 proteins that were causally associated with dementia causing diseases in Mendelian randomization analyses. Figures A-N include biomarkers for amyloid and tau and O-R for $\alpha$ synuclein pathways.

A)

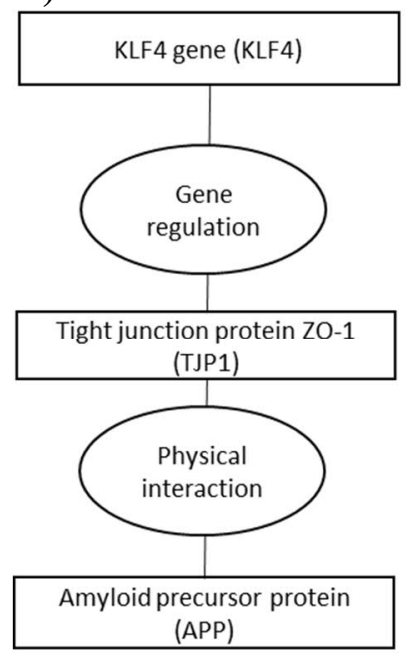

B)

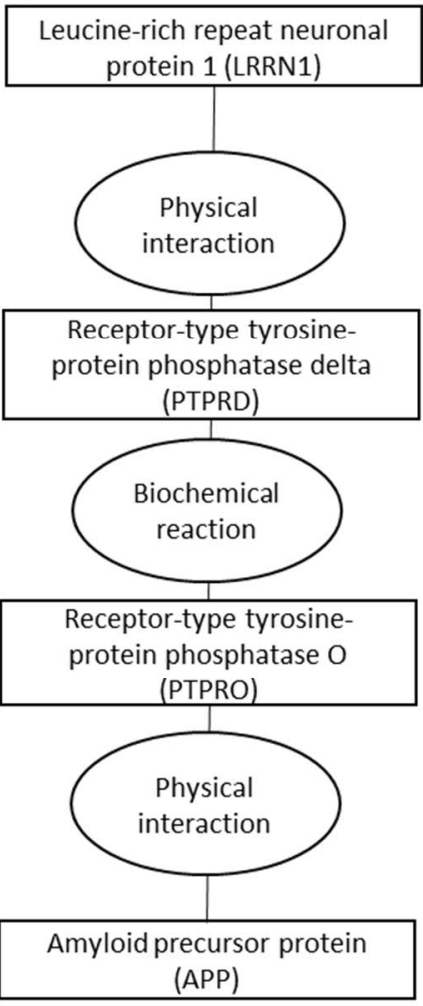

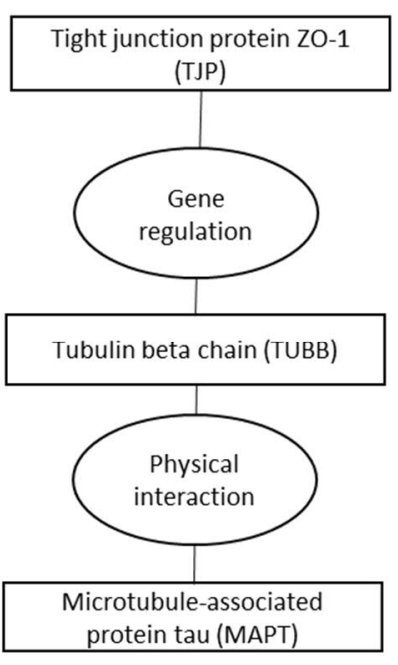
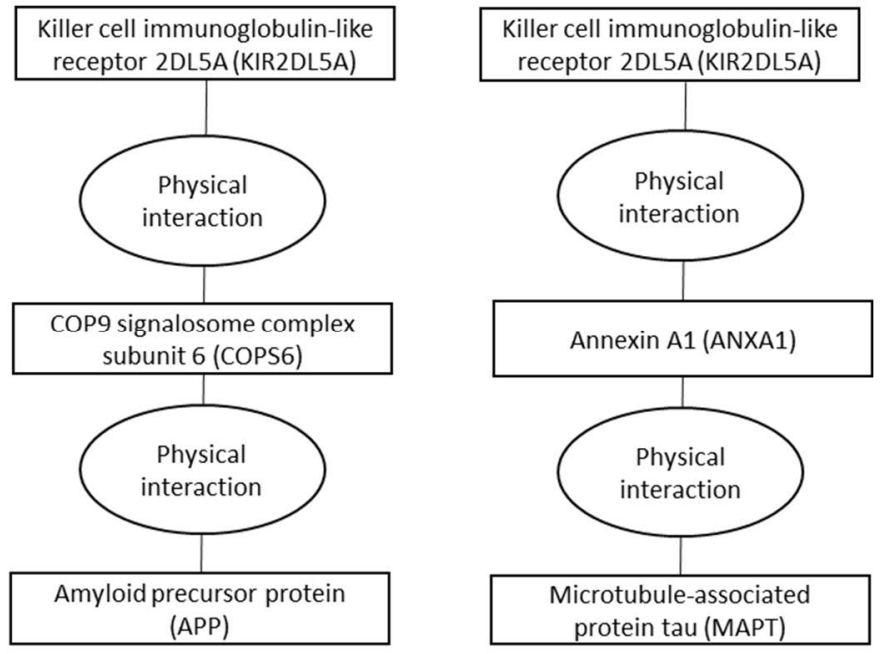

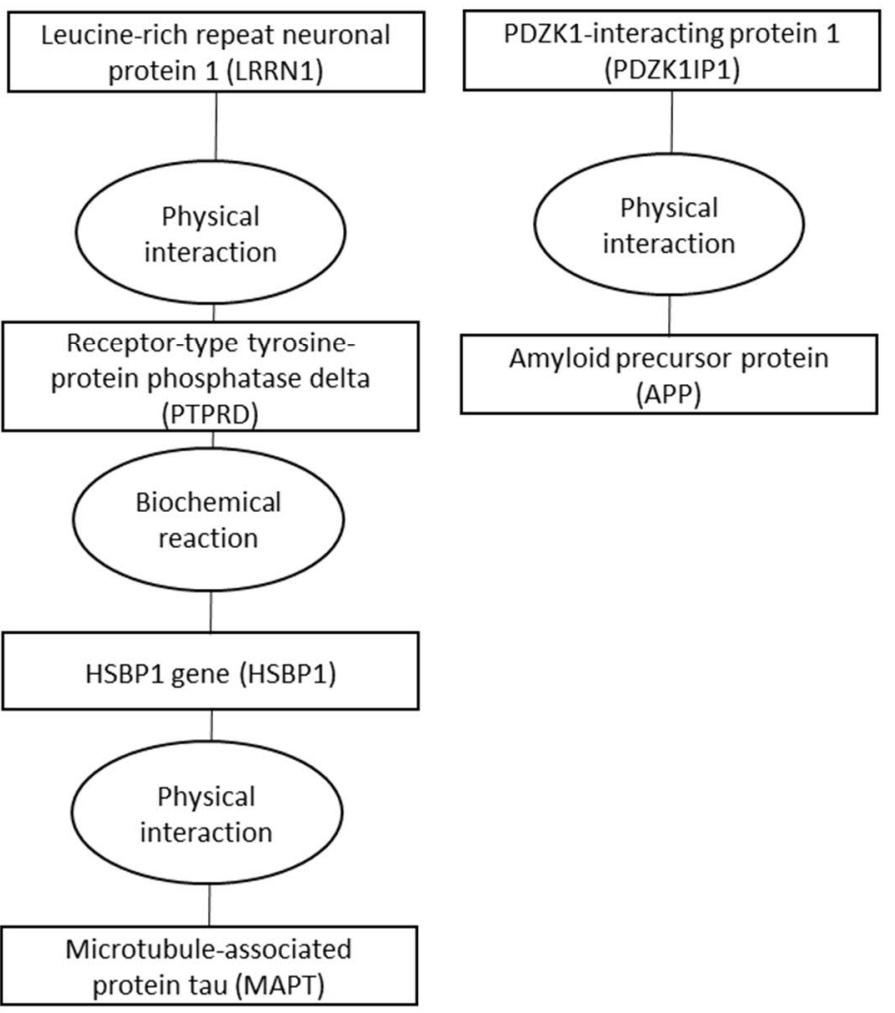

PDZK1-interacting protein 1 (PDZK1IP1)

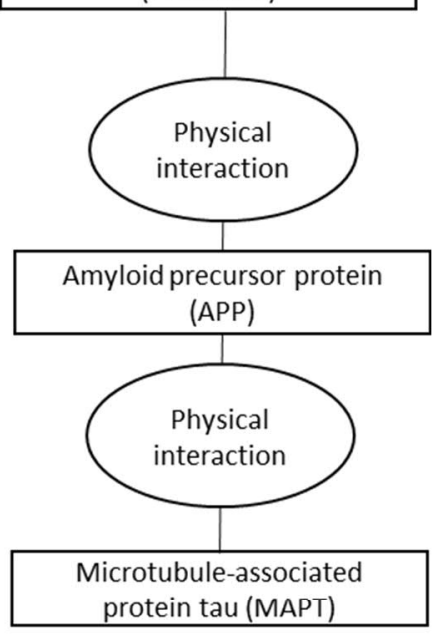


C)

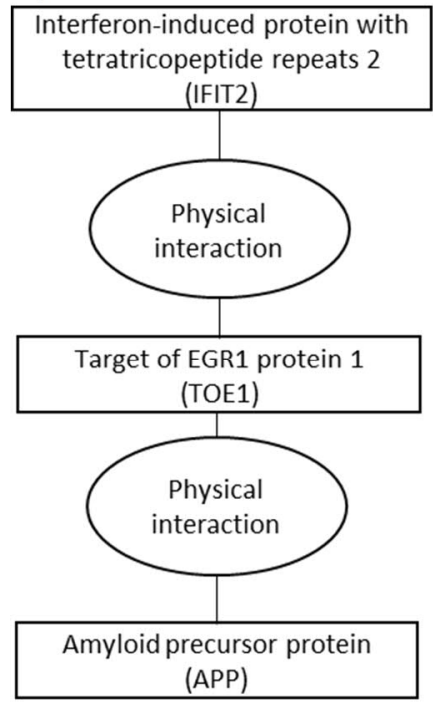

D)

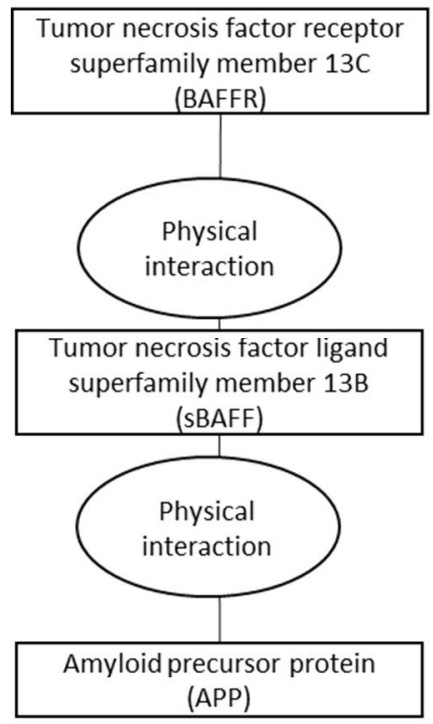

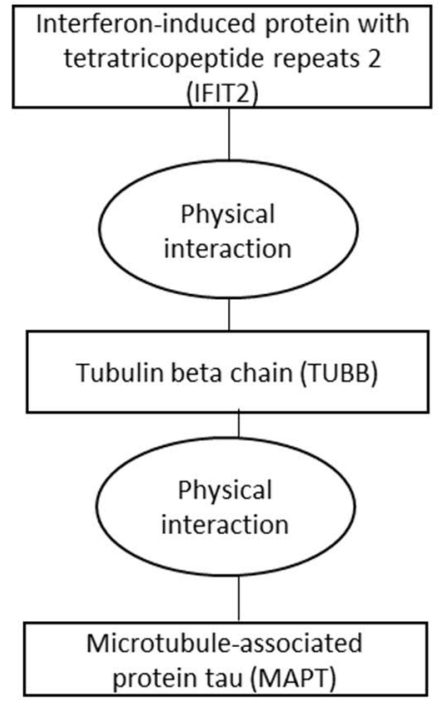
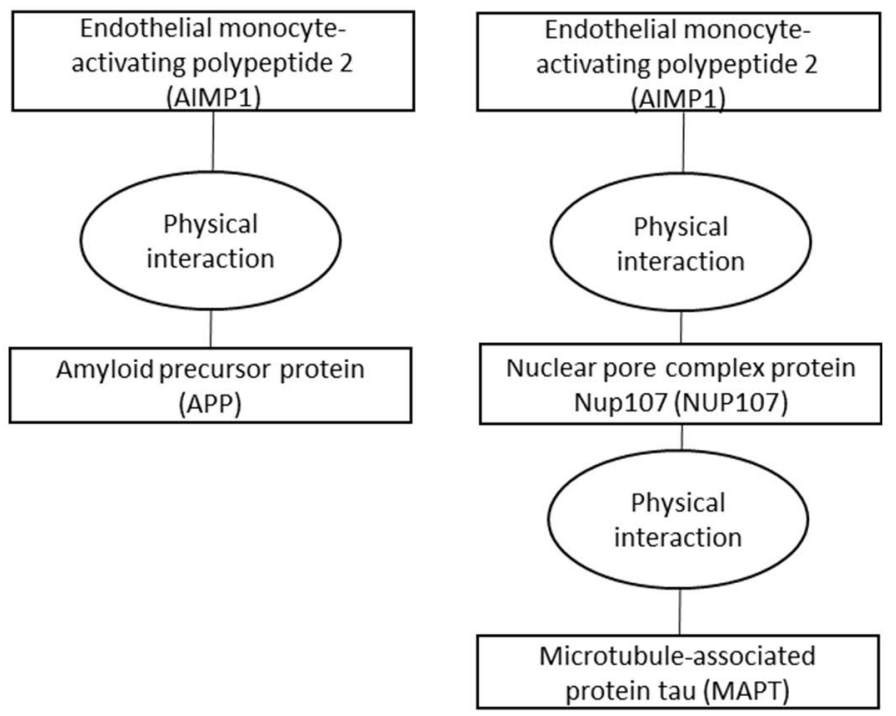
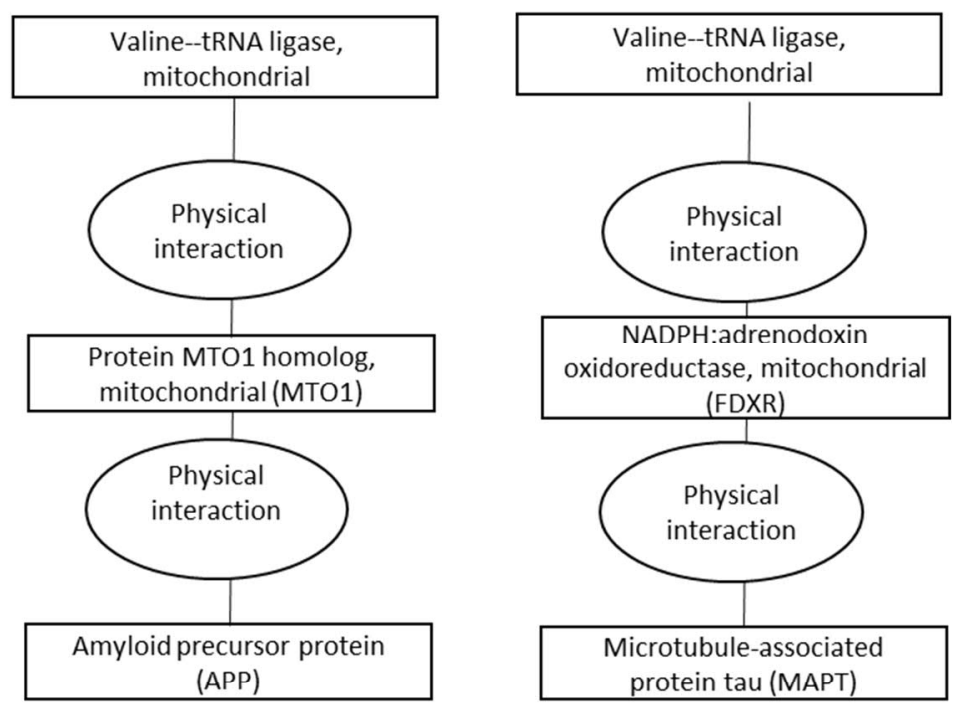
E)

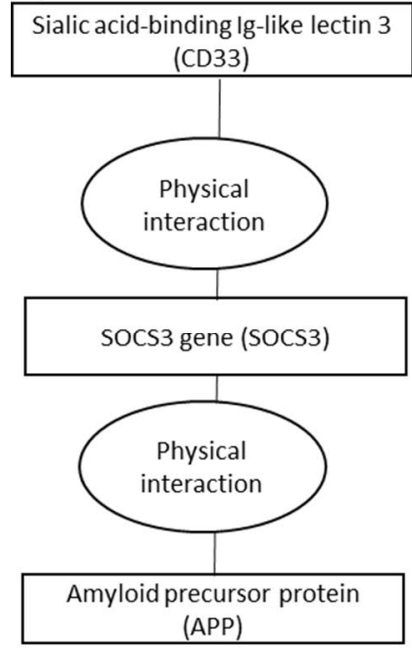

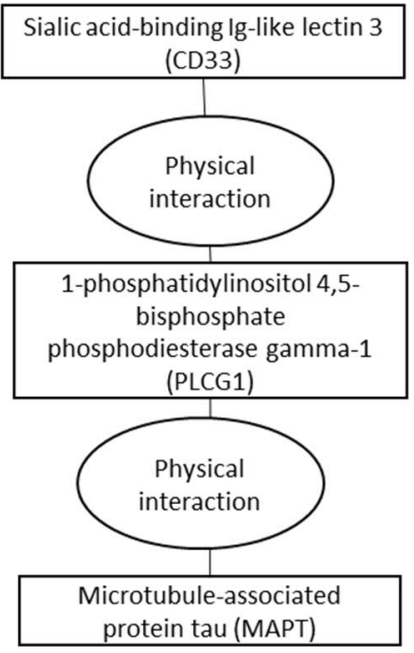

protein tau (MAPT)
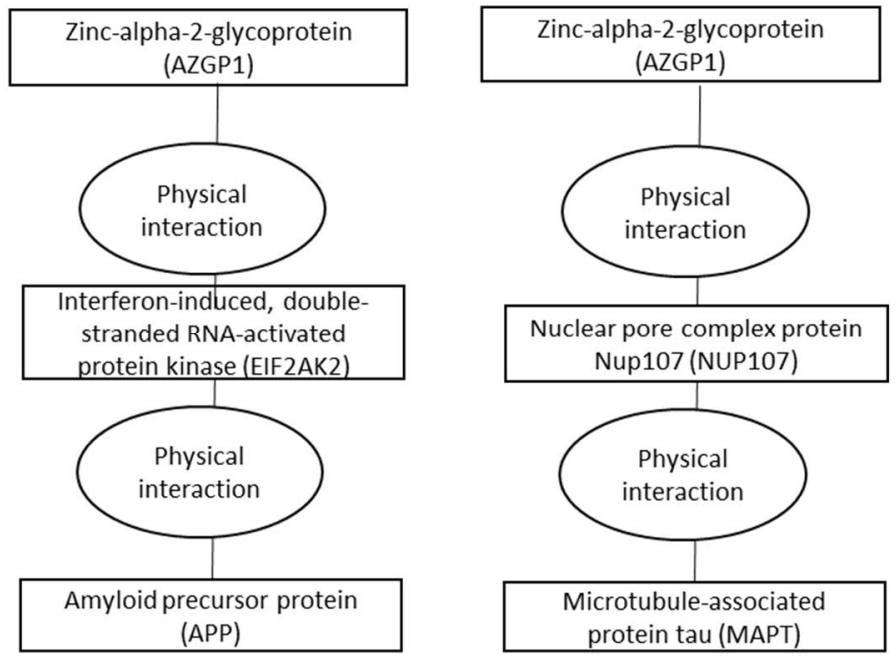

F)
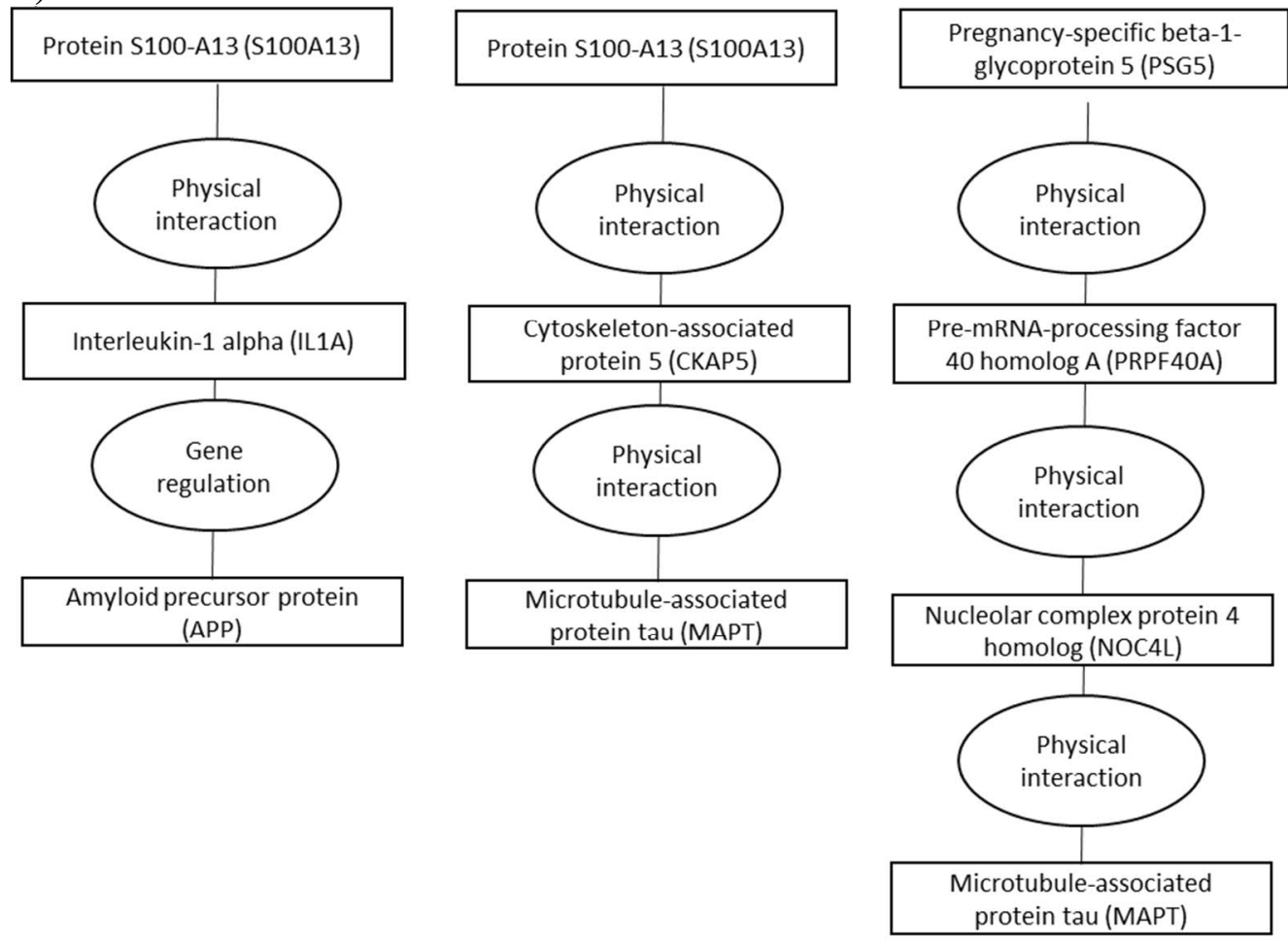
G)

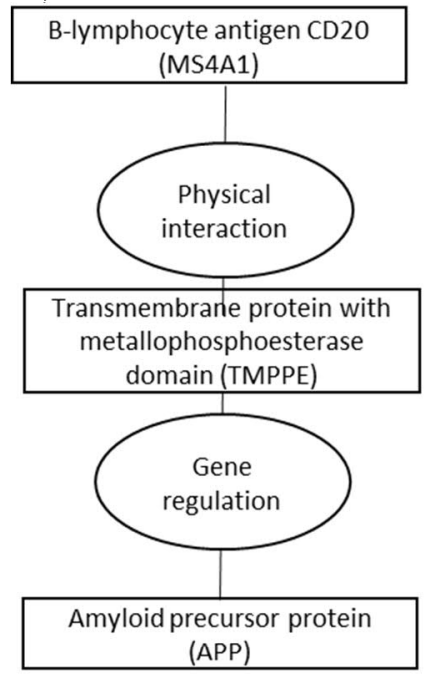

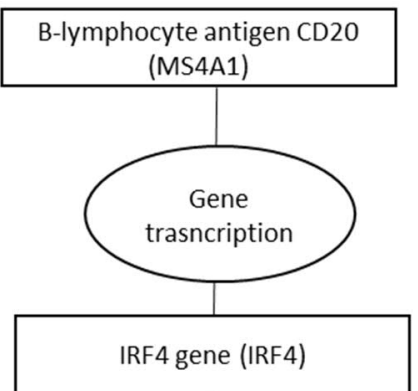

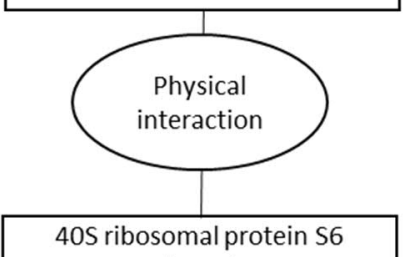

(RPS6)

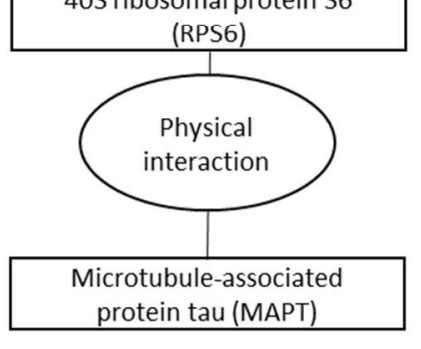

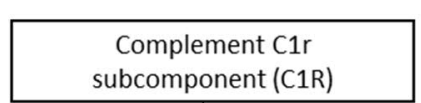

subcomponent (C1R)

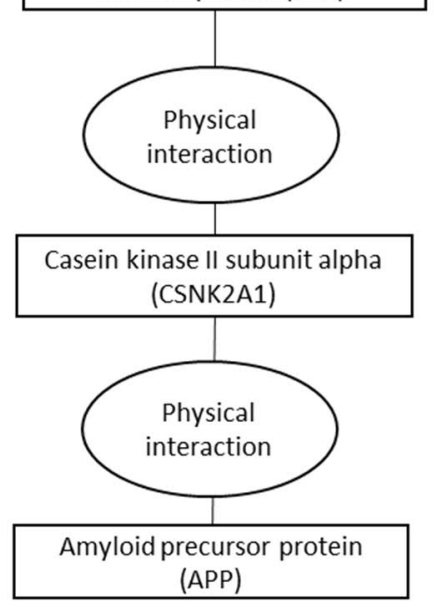

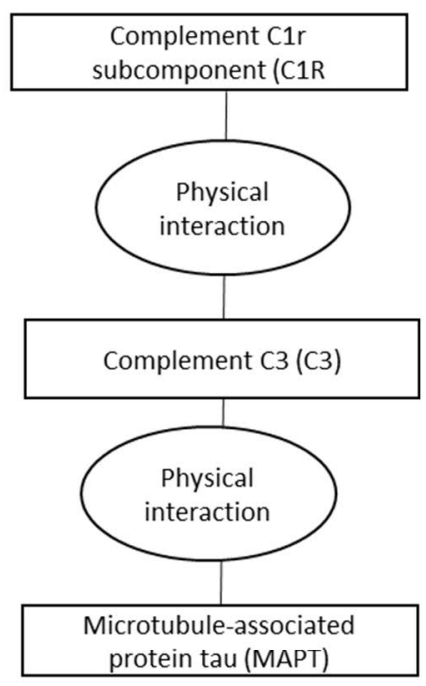

H)
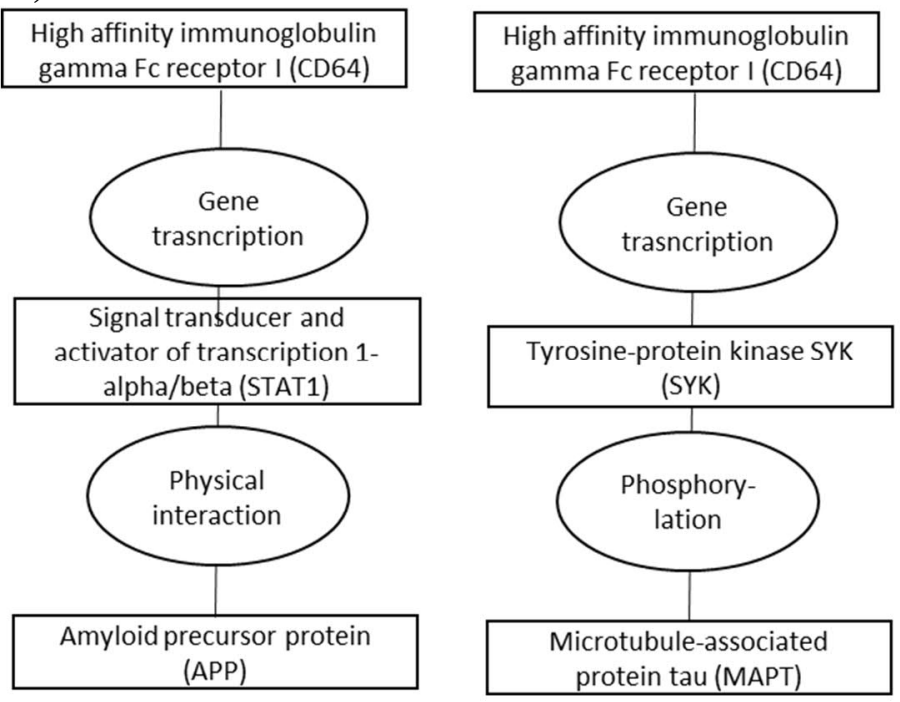

Receptor-type tyrosine-protein phosphatase C (CD45)

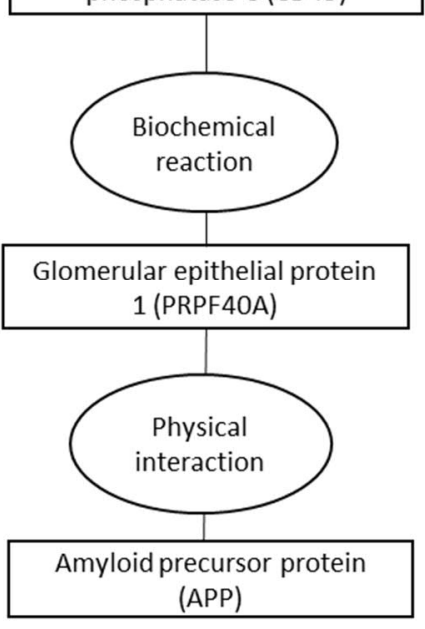

Receptor-type tyrosineprotein phosphatase C (CD45)

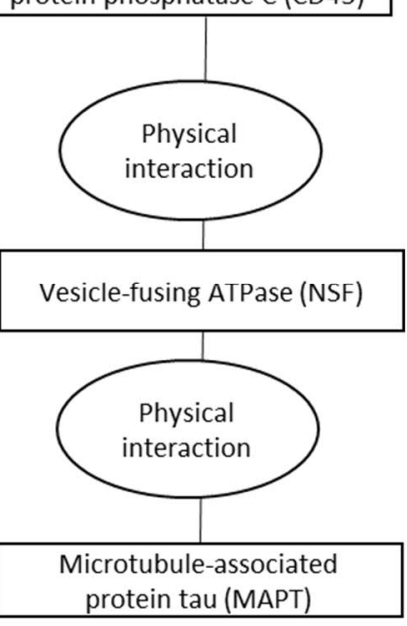


I)

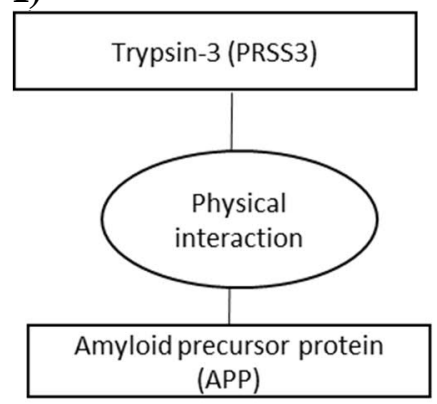

(APP)

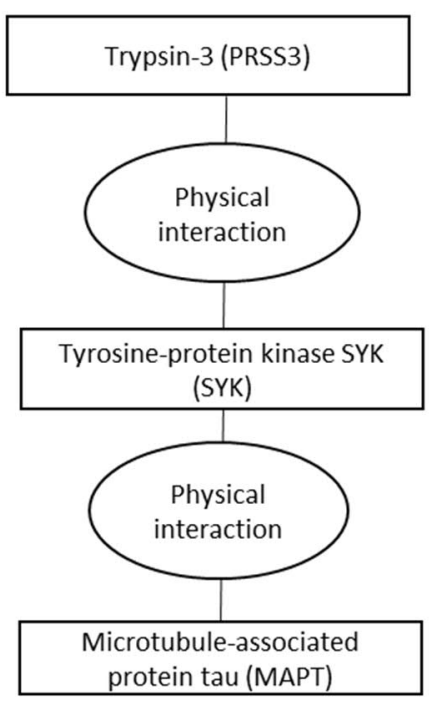

J)

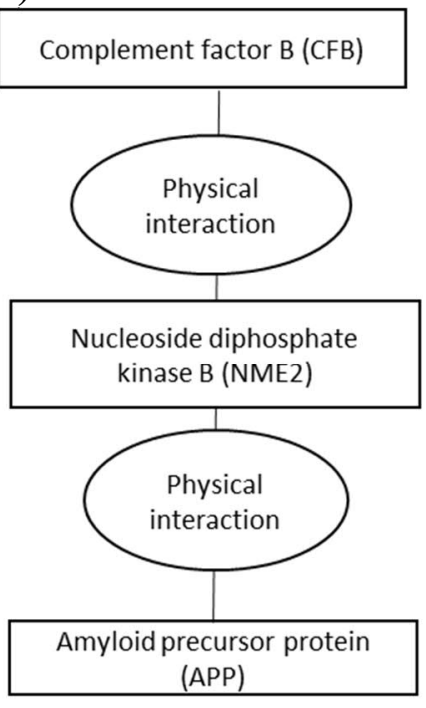

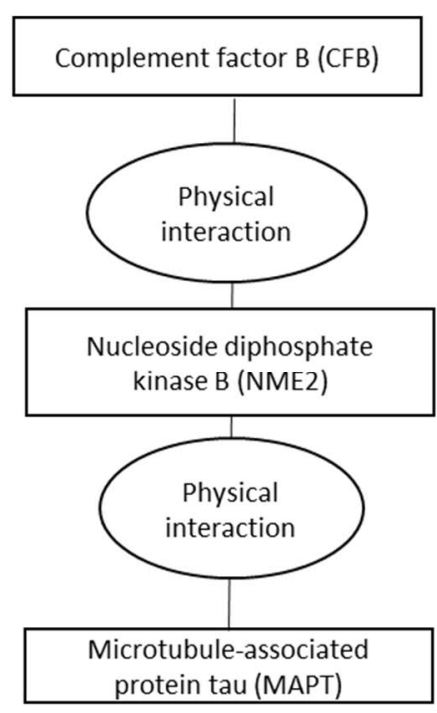

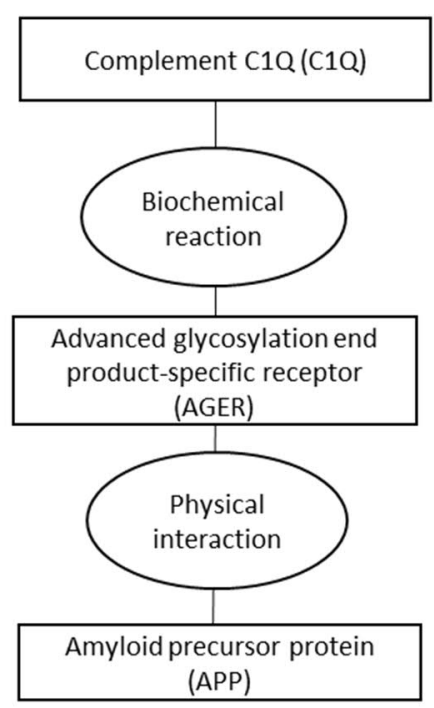

Receptor-type tyrosine-protein phosphatase C (CD45)

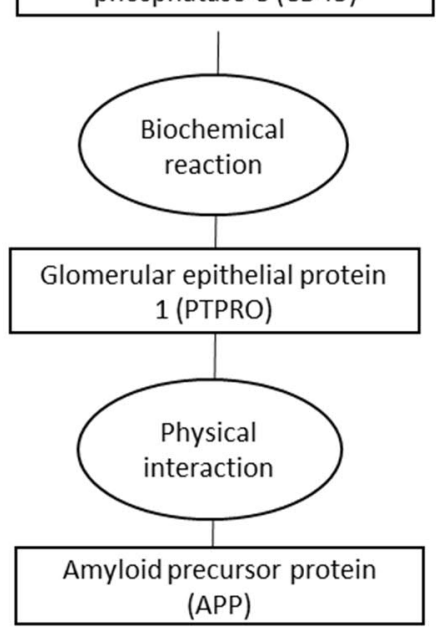

Complement C1Q (C1Q)

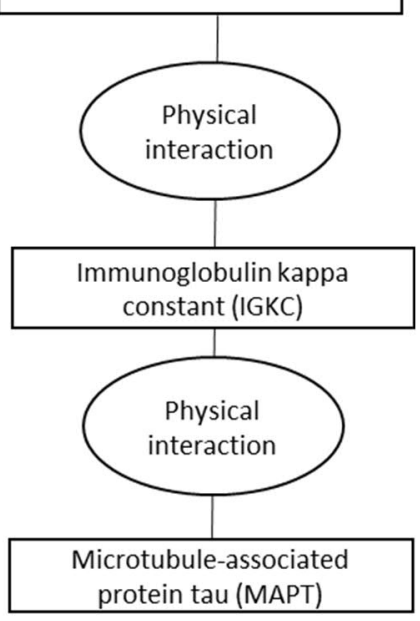

Receptor-type tyrosineprotein phosphatase C (CD45)

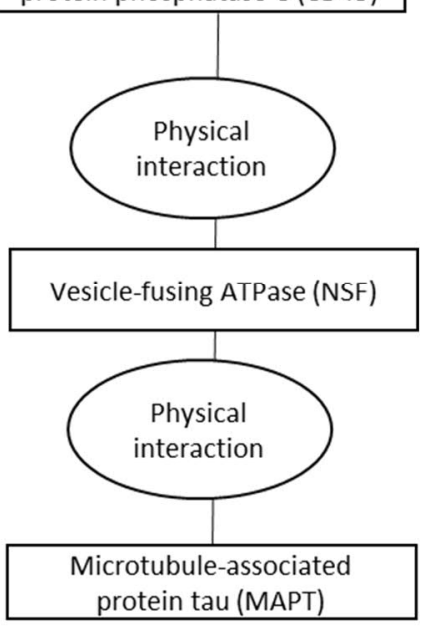


K)
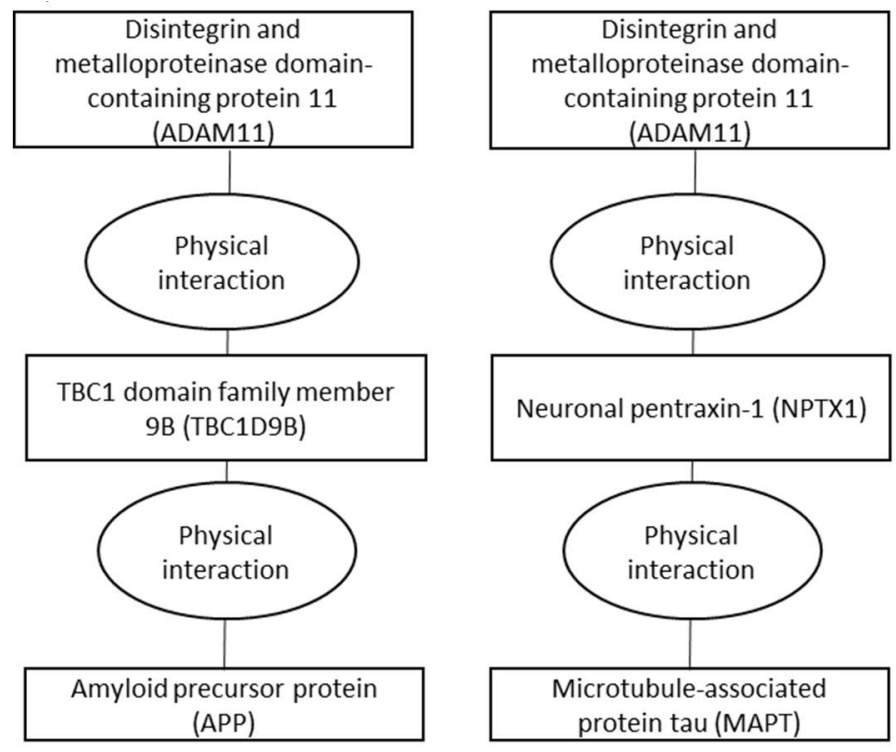

L)

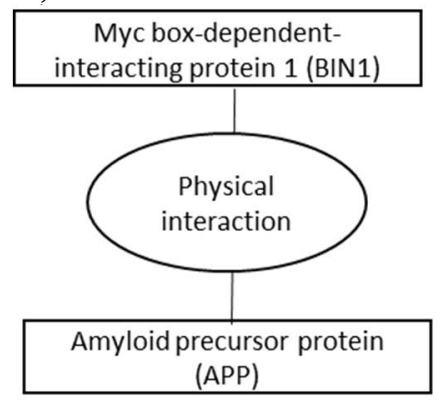

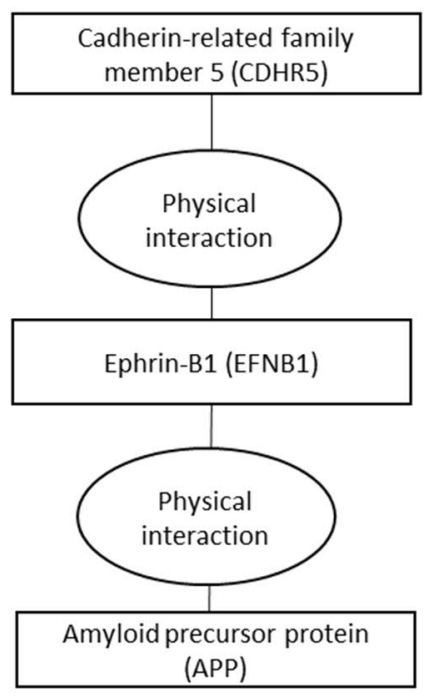

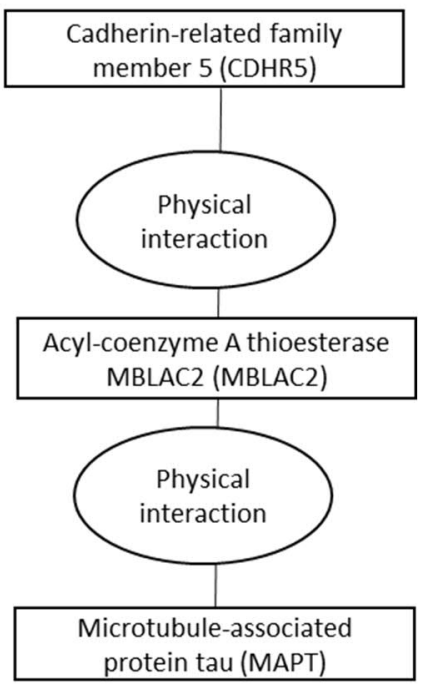

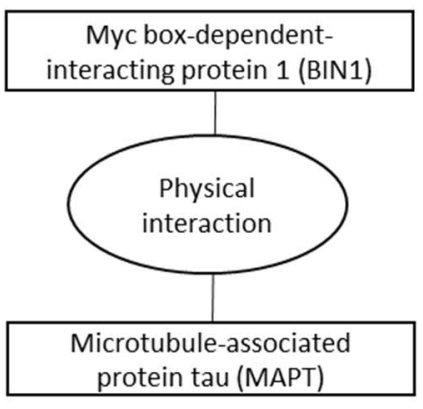

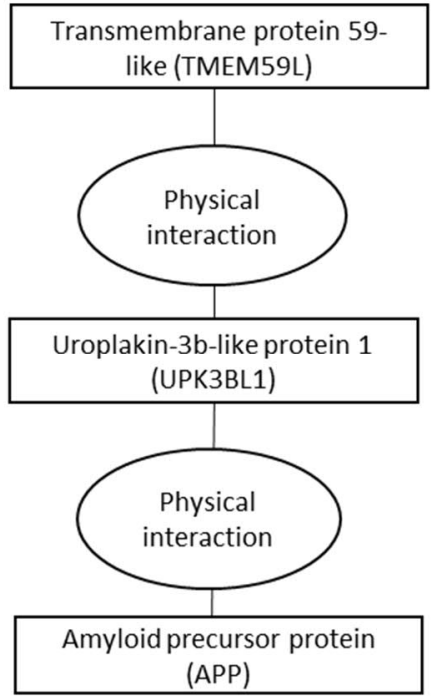

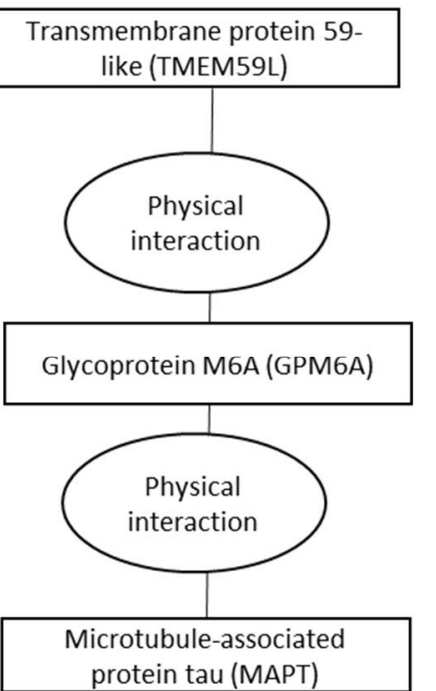


M)
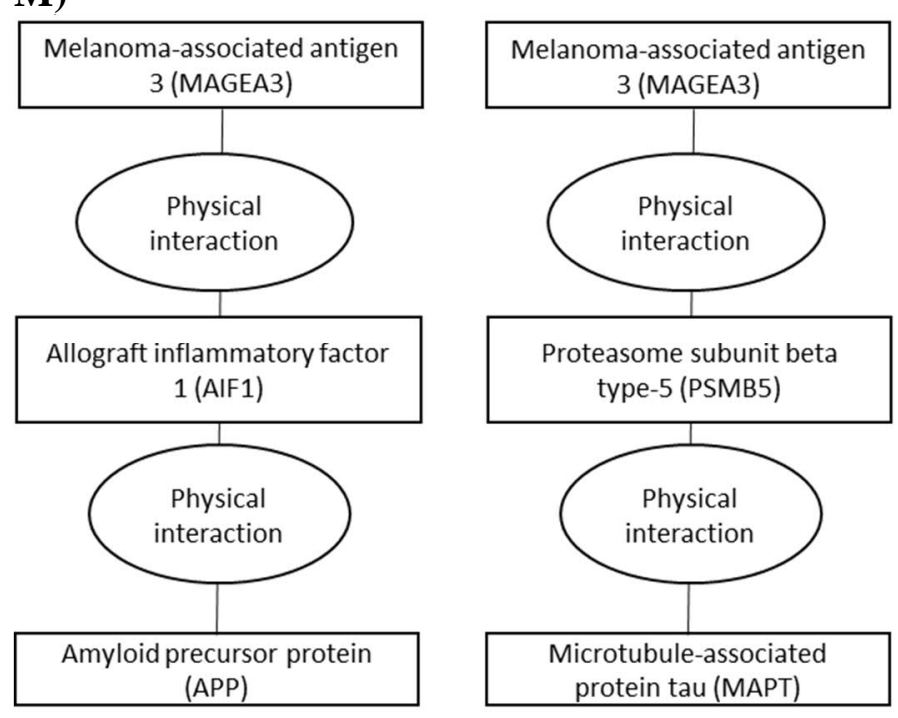

N)
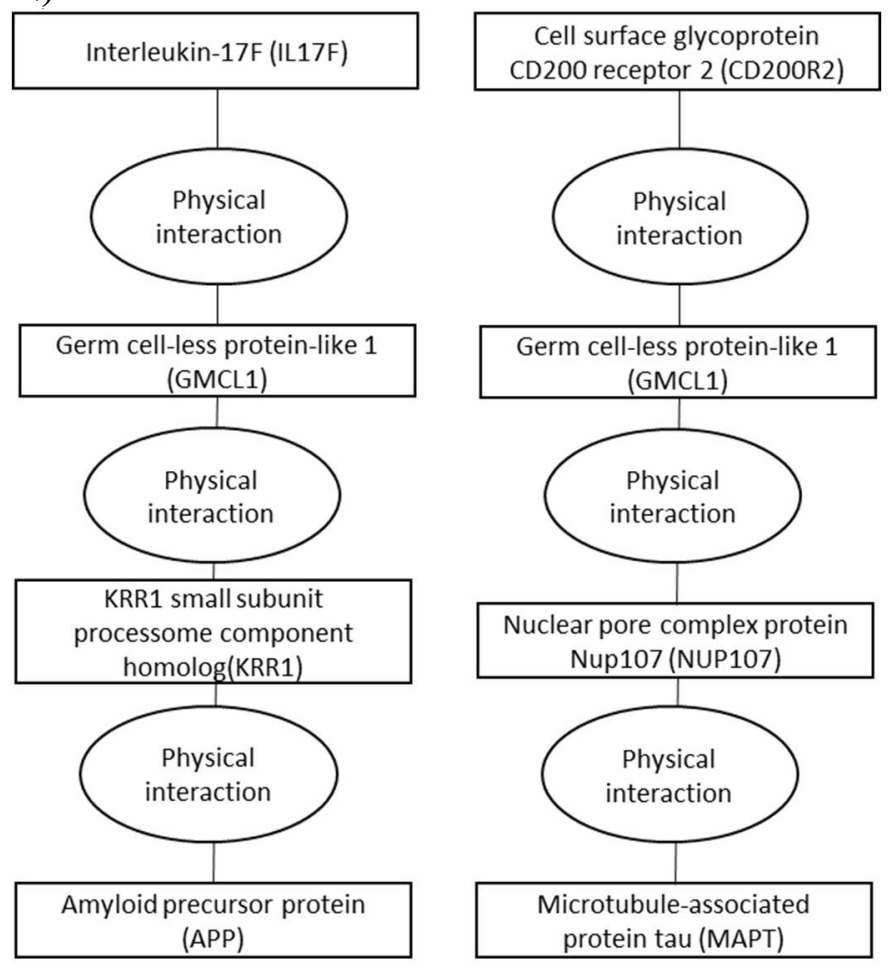
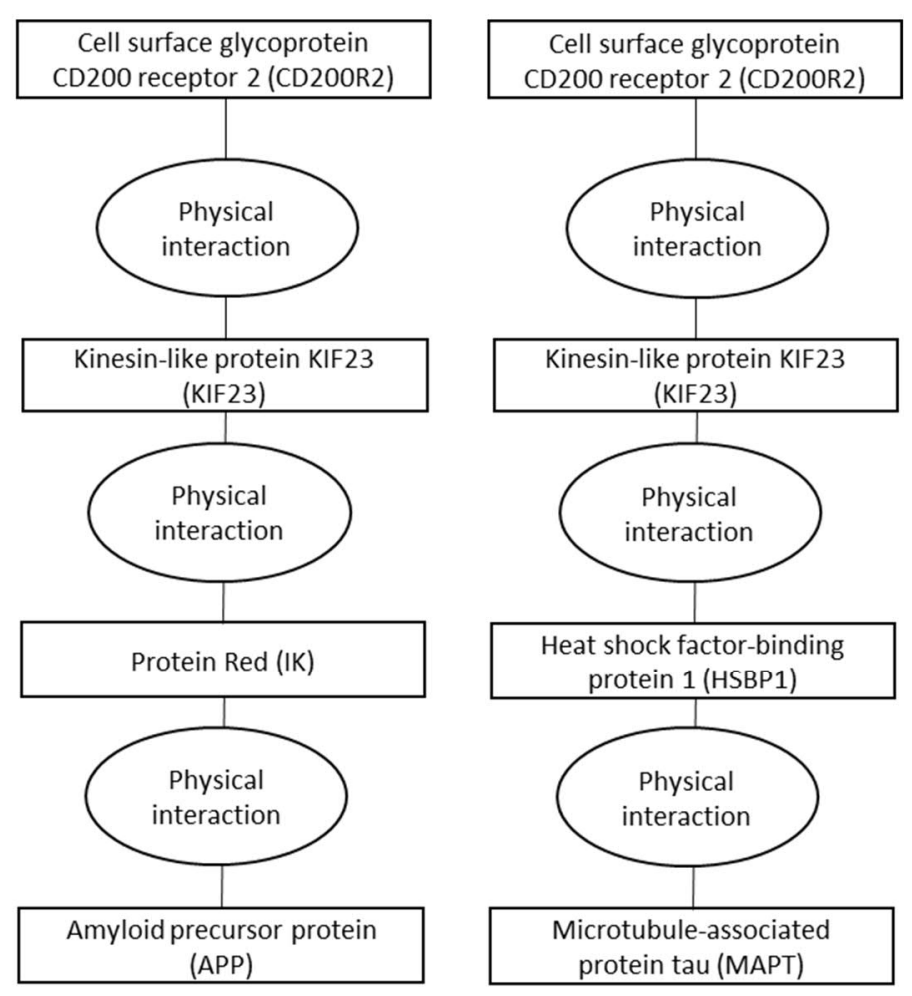
O)

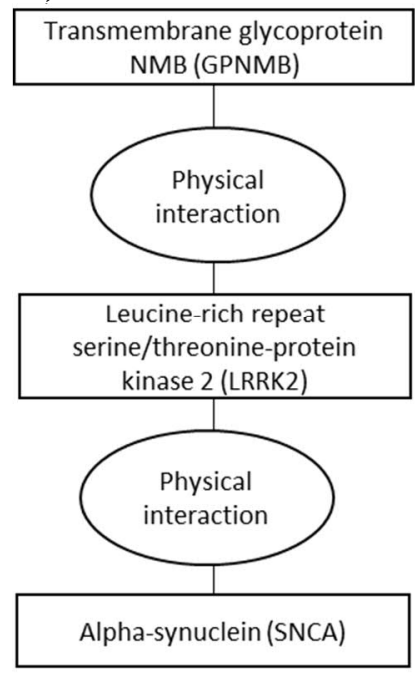

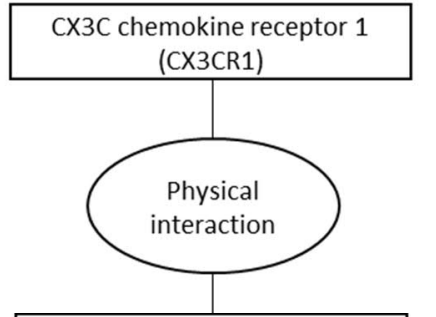
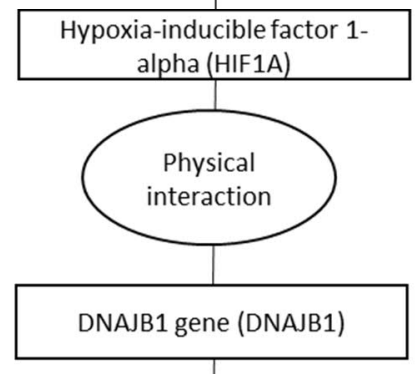

Physical

interaction

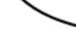

Alpha-synuclein (SNCA)

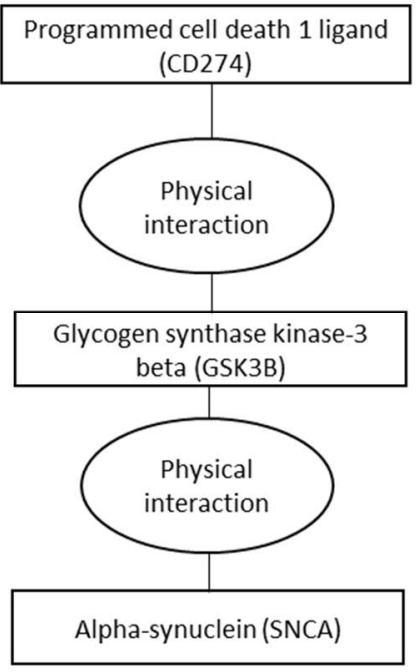

Integrin alpha-M (ITGAM)

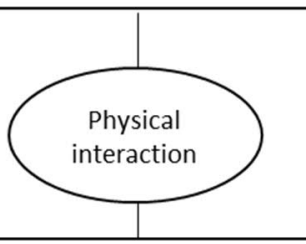

Phosphate carrier protein, mitochondrial (SLC25A3)

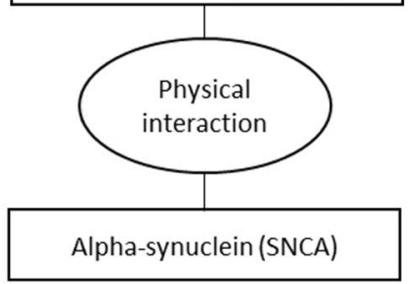

\section{P)}

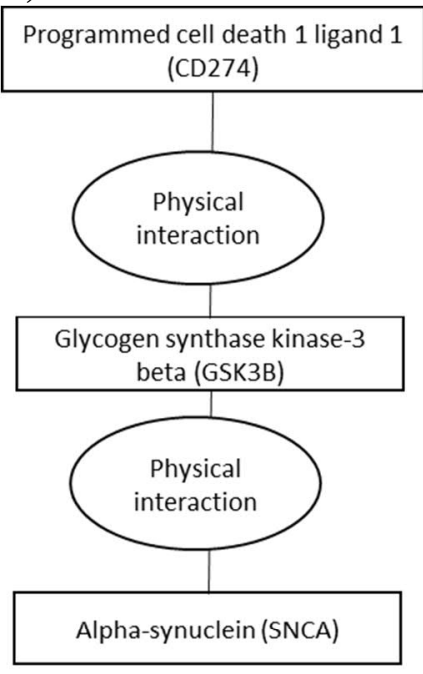

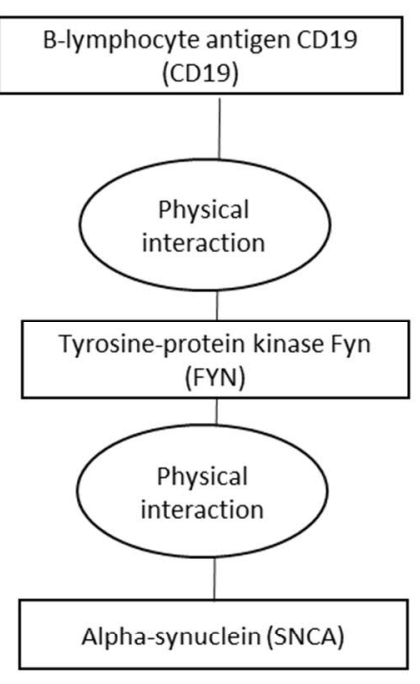

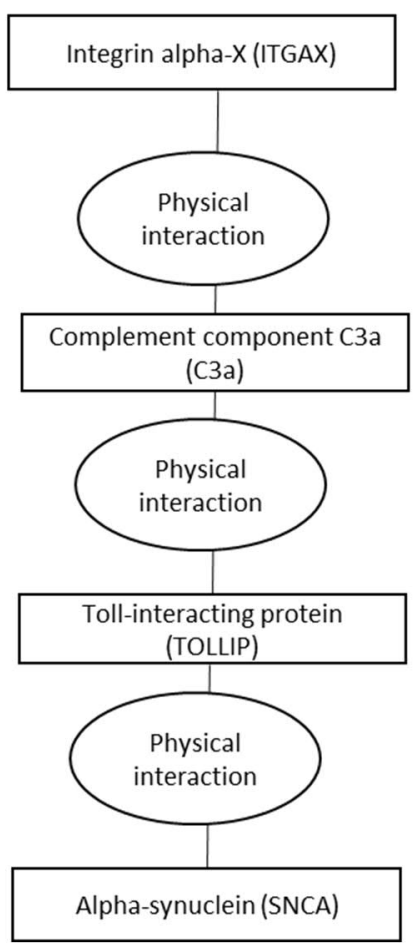

CD27 antigen (CD27)

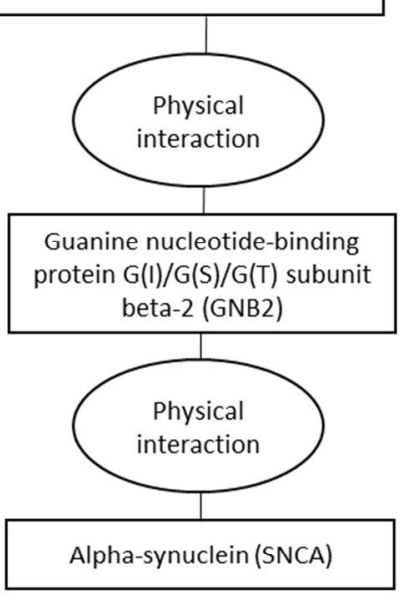

Alpha-synuclein (SNCA) 
Q)
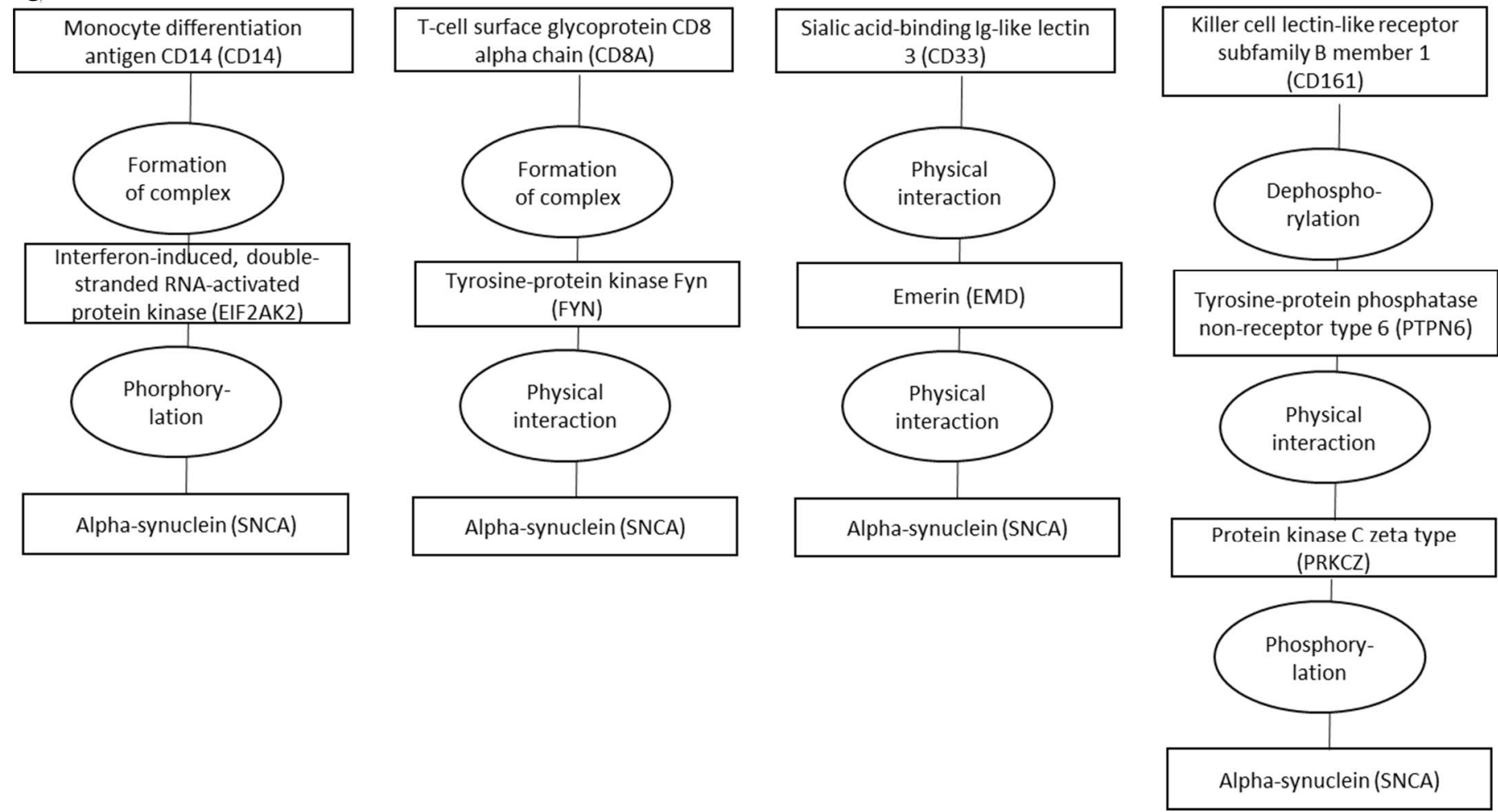

\section{R)}
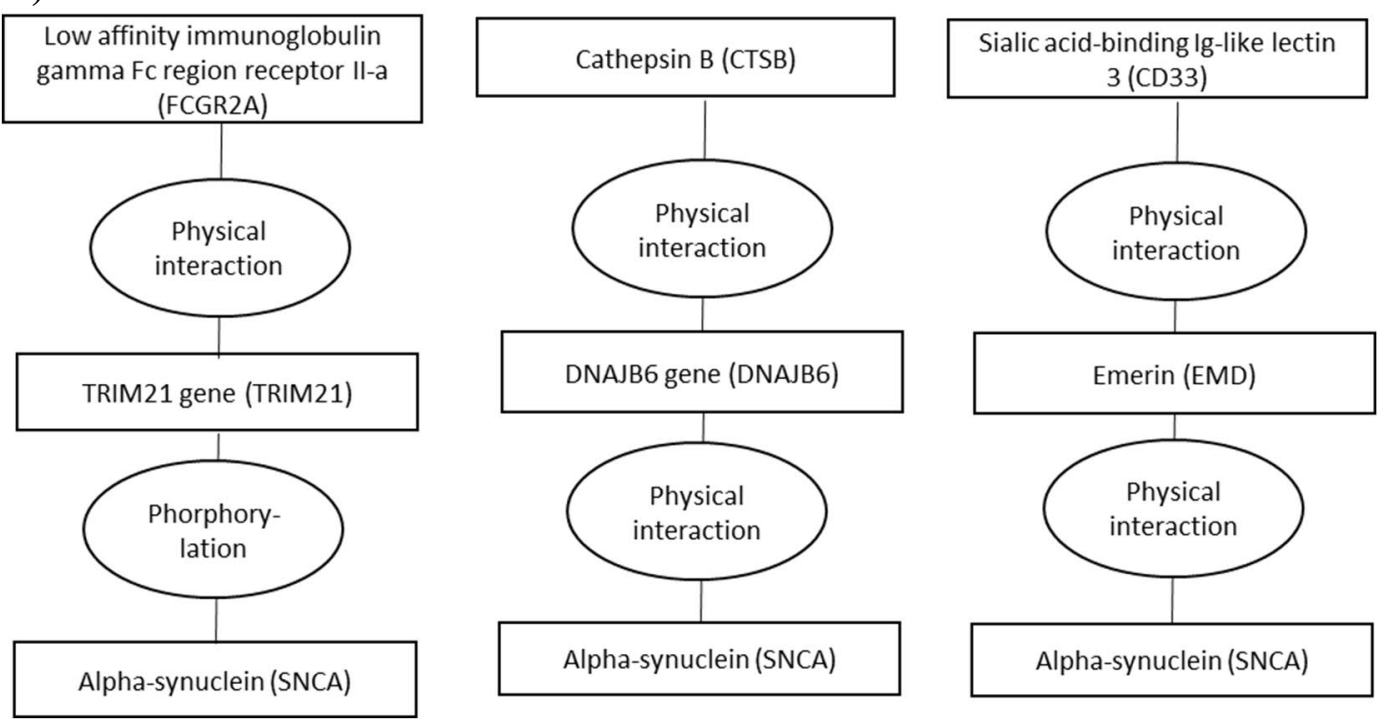
medRxiv preprint doi: https://doi.org/10.1101/2022.02.17.22271136; this version posted February 18, 2022. The copyright holder for this preprint (which was not certified by peer review) is the author/funder, who has granted medRxiv a license to display the preprint in

It is made available under a CC-BY-NC-ND 4.0 International license.

\section{Supplementary methods}

\section{Mendelian randomization analyses}

The SNPs for biomarkers and outcomes were searched from MR-base database. ${ }^{1}$ Immune system and BBB search terms were identified using identifiers of cell types, receptors, proteins, and genes. The identifiers were searched from several publications ${ }^{2-10}$ and from Uniprot ${ }^{11}$ with search terms "immune" and "blood brain barrier". Outcomes included all cohorts with following dementiacausing diseases: all types of Alzheimer's disease, Parkinson's disease, vascular dementia, frontotemporal dementia, dementia in general, and progression of dementia. Poor cognitive performance was chosen as an intermediate outcome. Two sample Mendelian randomization was used to analyse associations between biomarkers and outcomes. ${ }^{12}$ First analyses estimated effects with Wald ratio or inverse variance-weighted analyses. ${ }^{1}$ For biomarker-outcome pairs that passed FDR of 5\% and shared over two common SNPs we performed sensitivity analyses with weighted median, simple mode, MR Egger, and backward Mendelian randomization ${ }^{12}$ with TwoSampleMR and MRInstruments R packages.

To assess off-target effects of the observed causal biomarkers, we performed a phenome-wide Mendelian randomization analyses for each biomarker, using GWAS summary statistics for 210 UK Biobank endpoints conducted by Neale lab and with recognized dementia risk factors. ${ }^{5,13}$ All analyses used European ancestry as reference, clumping cut-off $\mathrm{R} 2=0.01$ and a $10,000 \mathrm{~kb}$ window. LD proxies were searched with $\mathrm{R} 2=0.6$ and a proxy split size of 500 . The biomarkers and outcome alleles were harmonized by inferring from positive strand alleles using allele frequencies for palindromes. We used statistical software R (3.6.0 and 4.1.0) for these analyses. Novelty of our Mendelian randomization findings was evaluated with Pubmed search using following search terms: (Mendelian randomization) AND (dementia OR Alzheim* OR Parkin* OR cognitive decline) AND (Entrez gene symbol OR Uniprot protein identifier) without limitations.

\section{Plasma proteins and Whitehall II study}

Plasma protein measurements in Whitehall II study were available for 6,235 individuals of whom 310 developed dementia. The participants are linked to the National Health Services (NHS) Hospital Episode Statistics (HES) database, and the British National mortality register using individual NHS identification numbers for linkage. ${ }^{14}$ The NHS provides nearly complete health care coverage for all individuals legally resident in the UK. Incident dementia was defined using the WHO International Classification of Diseases, version 10 (ICD-10) codes F00, F01, F03, G30, and G31 and ICD-9 codes 290.0-290.4, 331.0-331.2, 331.82, and 331.9. We also conducted informant interviews and checked participants' medications at each screening (in 1996-1998, 2011-2013 and 2016-2017) for dementia-related medication. Sensitivity and specificity of dementia assessment based on HES data is 0.78 and $0.92 .{ }^{15}$

The plasma proteins were measured using the SomaScan version 4.0 and 4.1 assays. ${ }^{4,16,17}$ The assays were validated against external reference population and protein-specific conversion coefficients were used to balance the technical differences between versions 4.0 and 4.1. The 
medRxiv preprint doi: https://doi.org/10.1101/2022.02.17.22271136; this version posted February 18, 2022. The copyright holder for this preprint (which was not certified by peer review) is the author/funder, who has granted medRxiv a license to display the preprint in

It is made available under a CC-BY-NC-ND 4.0 International license.

analyses used plasma samples measured in $1995 / 1997$ and stored in $0.25 \mathrm{~mL}$ aliquots at $-80^{\circ} \mathrm{C}$. Earlier studies describe performance of the SomaScan assay and the modified aptamer binding in detail. $^{4,16,17}$ In brief, the assay uses a mix of thousands of slow off-rate modified aptamers (SOMAmers) that bind to proteins in participants' plasma samples where the specificity is ensured with two-step process analogous to a conventional immunoassay. The specificity of the aptamer reagents is good and has been tested in several ways. ${ }^{4,18,19}$ Median intra- and inter-assay coefficients of variation for SomaScan version 4 are $~ 5 \%$ and assay sensitivity is comparable to that of typical immunoassays, with a median lower limit of detection in the femtomolar range.

In the Whitehall II study, standard self-administered questionnaires provided data on age and sex. Using DNA extracted from whole blood, a standard PCR assay determined APOE genotype using the salting out method..$^{20,21}$ Two blinded independent observers read the genotype and any discrepancies were resolved by repeating the PCR analysis.

In analyses, proteins were transformed to a normal distribution by inverse rank-based normal transformation. The follow-up ended at onset of dementia, death, or $1^{\text {st }}$ of October 2019, which ever occurred first. Age, sex, and APOE adjusted Cox regression models estimated associations between the proteins and dementia. ${ }^{22}$ The proportionality assumption in all Cox models was assessed with Schoenfeld residuals and with log-log plots. ${ }^{22}$ We used statistical software R (3.6.0 and 4.1.0) for these analyses.

\section{Polygenic risk score and IPW analyses}

FinnGen Data Freeze 8 contains 339,233 individuals, and represents approximately 7\% of Finnish adult population. FinnGen is a collection of prospective epidemiological and disease-based cohorts, and hospital biobank samples and links genotypes by the unique national personal identification numbers to nationwide health registries, including the national hospital discharge (available from 1968), death (1969-), cancer (1953-) and medication reimbursement (1964-) and purchase (1995-) registries. The registry-based follow-up ended on Dec 31, 2019. Alzheimer's disease was defined with ICD-10 codes under F00 and G30, ICD-9 codes under 3310, ICD-8 code under 29010, and medication purchase code N06D. Vascular dementia was defined with ICD-10 codes under F01 and ICD-9 codes under 4378. Parkinson's disease was defined with ICD-10 codes under G20, ICD-9 codes under 3320A, ICD-8 code under 34200, and reimbursements code 110.

FinnGen samples were genotyped with Illumina and Affymetrix arrays (Illumina Inc., San Diego, and Thermo Fisher Scientific, Santa Clara, CA, USA), and genotype calls were made with the GenCall or zCall (for Illumina) and the AxiomGT1 algorithm for Affymetrix data. Individuals with ambiguous gender, high genotype missingness (>5\%), excess heterozygosity (+-4SD) and nonFinnish ancestry were excluded, as well as all variants with high missingness ( $>2 \%)$, low HardyWeinberg equilibrium p-value $(<1 \mathrm{e}-6)$ and minor allele count $(\mathrm{MAC}<3)$. Array data pre-phasing was carried out with Eagle 2.3.5.3 with the number of conditioning haplotypes set to 20,000.

Genotype imputation was done using the population-specific SISu v3 imputation reference with 
medRxiv preprint doi: https://doi.org/10.1101/2022.02.17.22271136; this version posted February 18, 2022. The copyright holder for this preprint (which was not certified by peer review) is the author/funder, who has granted medRxiv a license to display the preprint in

It is made available under a CC-BY-NC-ND 4.0 International license .

81 3,775 high-coverage (25-30x) whole-genome sequences in Finns, described in detail at https://doi.org/10.17504/protocols.io.xbgfijw.

The PRS were constructed from SNPs that were associated with the Mendelian randomization causal 126 biomarkers for dementias. The SNPs were LD-pruned with clumping cut-off $\mathrm{R} 2=0.01$ in 500kb window with $\mathrm{R}$ package TwoSampleMR. The available SNPs were searched from FinnGen genotypes and the final scores were generated with PLINK v2.00aLM3 by calculating the SNP-biomarker beta weighted sum of risk alleles for each SNP. The PRS was scaled to zero mean and unit variance, and we analysed its phenome-wide associations across 2401 disease endpoints. The association between PRS and endpoints were studied with logistic regression, adjusting for birth year, sex, and ten first principal components of ancestry.

We used IPW analyses to emulate randomized control trials on the effect of preventive antiinflammatory medication on risk of dementias in FinnGen. ${ }^{24}$ These analyses included participants aged over 45 without any dementia-causing disease at baseline $(\mathrm{N}=90,512)$ ATC codes for antiinflammatory medication use were searched from medication purchase registry that starts from 1995. To simulate trial design and to avoid immortal time bias, each analyses included only new medication users. In IPW analyses, we assumed that when medication is initiated, it is continued until end of follow-up to simulate intention to treat analyses and thus to provide conservative estimates. The baseline variables in IPW analyses were birth year, sex, ten principal components of ancestry and time-varying variables statin, ACE-blocker, AT-blocker, renin-blocker, calcium channel blocker, any diuretic, insulin, metformin, other diabetes drug, depression medication, antipsychotic, and anticoagulant use as well as time varying any cancer, myocardial infarction, atrial fibrillation, heart failure, venous thromboembolism, ischemic stroke, intracerebral haemorrhage, subarachnoid haemorrhage, obesity, sleep apnoea, and chronic obstructive pulmonary diseases, with informative censoring included. The positive control analyses on statin medication used these same variables but did not include statin as time-varying covariate. We used R (4.1.2) for these analyses.

\section{HLA analyses}

The analyses were done using imputed HLA alleles, imputed with high accuracy using a Finnishspecific reference panel, which has been previously described in detail. ${ }^{25}$ After filtering based on a HLA carrier frequency of $\geq 0.01$ and posterior probability of $\geq 0.6$, we assessed the association between HLA alleles and dementias and autoimmune diseases using logistic regression, adjusting for birth year, sex, and ten first principal components of ancestry.

\section{Pathway analyses}

KEGG pathway analysis was used for studying the effect of the biomarkers on validated pathways with Generally Applicable Gene-set Enrichment (GAGE), ${ }^{26}$ using Mendelian randomization Wald 
medRxiv preprint doi: https://doi.org/10.1101/2022.02.17.22271136; this version posted February 18, 2022. The copyright holder for this preprint (which was not certified by peer review) is the author/funder, who has granted medRxiv a license to display the preprint in It is made available under a CC-BY-NC-ND 4.0 International license.

120 ratios or inverse variance weight betas and p-values as input for expression ratios. GO term

121 enrichment analyses were done with ClueGO version 2.5.8 ${ }^{27}$ in Cytoscape version 3.7.2. ${ }^{28}$ The

122 hypergeometric test used all the 76 plasma proteins or surface receptors of the 126 biomarkers as

123 input and all immune system and BBB related proteins from Uniprot $^{11}$ as background, with

124 correction for 5\% FDR. The shortest interaction path analyses were done with ConsensusPathDB, ${ }^{29}$

125 a web based analysis tool containing a range of biomedical databases. ConsensusPathDB was used

126 to decipher the potential common pathways between the biomarkers and amyloid $\beta$, tau protein, and

$127 \alpha$-synuclein.

128

129 Open Targets analyses

130 Medications that change the levels of the 126 biomarkers were searched from Open Targets

131 database using Uniprot protein and Entrez gene symbols.

132

133

134

135

136

137

138

139

140

141

142

143

144

145

146

147

148

149

150

151

152 
medRxiv preprint doi: https://doi.org/10.1101/2022.02.17.22271136; this version posted February 18, 2022. The copyright holder for this preprint (which was not certified by peer review) is the author/funder, who has granted medRxiv a license to display the preprint in

It is made available under a CC-BY-NC-ND 4.0 International license .

\section{Supplementary references}

1. Hemani, G., et al. The MR-Base platform supports systematic causal inference across the human phenome. Elife 7(2018).

2. Akiyama, H., et al. Inflammation and Alzheimer's disease. Neurobiology of aging 21, 383421 (2000).

3. Dendrou, C.A., Petersen, J., Rossjohn, J. \& Fugger, L. HLA variation and disease. Nat Rev Immunol 18, 325-339 (2018).

4. Lindbohm, J.V., et al. Plasma proteins, cognitive decline, and 20-year risk of dementia in the Whitehall II and Atherosclerosis Risk in Communities studies. Alzheimer's \& dementia : the journal of the Alzheimer's Association (2021).

5. Livingston, G., et al. Dementia prevention, intervention, and care: 2020 report of the Lancet Commission. Lancet (London, England) 396, 413-446 (2020).

6. O'Brien, J.T. \& Thomas, A. Vascular dementia. Lancet (London, England) 386, 1698-1706 (2015).

7. Sweeney, M.D., Zhao, Z., Montagne, A., Nelson, A.R. \& Zlokovic, B.V. Blood-Brain Barrier: From Physiology to Disease and Back. Physiological Reviews 99, 21-78 (2019).

8. Tan, E.K., et al. Parkinson disease and the immune system - associations, mechanisms and therapeutics. Nat Rev Neurol 16, 303-318 (2020).

9. Theofilopoulos, A.N., Kono, D.H. \& Baccala, R. The multiple pathways to autoimmunity. Nat Immunol 18, 716-724 (2017).

10. Walker, K.A., et al. Large-scale plasma proteomic analysis identifies proteins and pathways associated with dementia risk. Nature Aging 1, 473-489 (2021).

11. UniProt, C. UniProt: a worldwide hub of protein knowledge. Nucleic acids research 47, D506-D515 (2019).

12. Holmes, M.V., Richardson, T.G., Ference, B.A., Davies, N.M. \& Davey Smith, G. Integrating genomics with biomarkers and therapeutic targets to invigorate cardiovascular drug development. Nat Rev Cardiol 18, 435-453 (2021).

13. http://www.nealelab.is/uk-biobank/.

14. Kivimaki, M., et al. Validity of Cardiovascular Disease Event Ascertainment Using Linkage to UK Hospital Records. Epidemiology (Cambridge, Mass.) 28, 735-739 (2017).

15. Sommerlad, A., et al. Accuracy of general hospital dementia diagnoses in England: Sensitivity, specificity, and predictors of diagnostic accuracy 2008-2016. Alzheimer's \& dementia : the journal of the Alzheimer's Association 14, 933-943 (2018).

16. Gold, L., et al. Aptamer-based multiplexed proteomic technology for biomarker discovery. PloS one 5, e15004 (2010).

17. Williams, S.A., et al. Plasma protein patterns as comprehensive indicators of health. Nature medicine 25, 1851-1857 (2019).

18. Emilsson, V., et al. Co-regulatory networks of human serum proteins link genetics to disease. Science (New York, N.Y.) 361, 769-773 (2018).

19. Tin, A., et al. Reproducibility and Variability of Protein Analytes Measured Using a Multiplexed Modified Aptamer Assay. The journal of applied laboratory medicine 4, 30-39 (2019).

20. Bolla, M.K., Wood, N. \& Humphries, S.E. Rapid determination of apolipoprotein E genotype using a heteroduplex generator. Journal of lipid research 40, 2340-2345 (1999).

21. Miller, S.A., Dykes, D.D. \& Polesky, H.F. A simple salting out procedure for extracting DNA from human nucleated cells. Nucleic acids research 16, 1215 (1988).

22. Cox, D.R. Regression Models and Life-Tables. Journal of the Royal Statistical Society.Series B (Methodological) 34, 187-220 (1972). 
medRxiv preprint doi: https://doi.org/10.1101/2022.02.17.22271136; this version posted February 18, 2022. The copyright holder for this preprint (which was not certified by peer review) is the author/funder, who has granted medRxiv a license to display the preprint in perpetuity.

202 23. Loh, P.R., et al. Reference-based phasing using the Haplotype Reference Consortium panel. 


\section{SNP}

rs12461065

$\$ 395908$

rs429358
rs429358

rs429358

rs 529358

rs438811

r55167

r55167

rs5167

rs5167
rs5167

r559325138

rs7412

rs7412

rs7412
rs7412
Beta exposure

\section{Exposure}

0.1574 Vallie-tRMAligase || id.prot-a-3191

0.3382 EMAP-2|| id:prot-c-2714_78_2

0.3499 Killer cell immunoglobulin-like receptor 2DL5A || id:prot-a-1643

1.1769 Leucine-rich repeat neuronal protein 1|| id:prot-a-1792

0.3527 PDZK1-interacting protein 1 || id:prot-a-2242

0.2148 Tight junction protein ZO-1 || id:prot-a-2983

-1.2435 Protein S100-A13 || id:prot-a-2616

0.2454 BAFF Receptor || id:prot-c-5383_14_3

0.3319 Endothelial monocyte-activating polypeptide 2 || id:prot-a-65

0.277 Melanoma-associated antigen 3|| id:prot-a-1828

0.2145 Transmembrane protein 59-like || id:prot-a-3014

.8678 Valine--tRNA ligase || id:prot-a-319

0.1436 Interferon-induced protein with tetratricopeptide

0.4743 Cadherin-related family member 5 || id:prot-a-490

0.7212 Cell surface glycoprotein CD200 receptor 2 || id:prot-a-424

-0.3721 Disintegrin and metalloproteinase domain-containing prote
Outcome

Alzheimer's disease (more excluded) || id:finn-a-AD_EXMOR

Alzheimer's disease || id:ieu-b-2

Alzheimer's disease (late onset) || id:ebi-a-GCST002245

Alzheimer's disease (late onset) || id:ebi-a-GCST002245

Alzheimer's disease (late onset) || id:ebi-a-GCST002245

Alzheimer's disease (late onset) || id:ebi-a-GCST002245

Alzheimer's disease (late onset) || id:ebi-a-GCST002245

Alzheimer's disease (late onset) || id:ebi-a-GCST002245

Alzheimer's disease (late onset) || id:ebi-a-GCST002245

Alzheimer's disease (late onset) || id:ebi-a-GCST002245

Alzheimer's disease (late onset) || id:ebi-a-GCST002245

Alzheimer's disease (more excluded) || id:finn-a-AD_EXMORE

Alalzheimer's disease (late onset) || id:ebi-a-GCST002245

Alzheimer's disease (late onset) || id:ebi-a-GCST002245

|| ic Alzheimer's disease (late onset) || id:ebi-a-GCST (late onset) || id:ebi-a-GCST02245

Alzheimer disease (more excluded) || id:finn-a-G6__ALZHEIMER_E
Chromosome

Position

45102050

45380961

45411941

45411941

45411941

45418790

45448465

45448465

45448465

45448465

44980181

45416291

45412079

45412079

45412079
44921921
Distance from start of APOE Distance from end of APOE

306961 -

28050 .

2930 -

2930 -

9779

39454

39454

39454

39454

428830

7280

3068

3068 -

3068
487090

709

709
709

709

6140

35815
3515
35815

35815

32469

3641

57

490729 
Supplementary File 2. Drugs available in the Open Targets database that act on the biomarkers that associated with dementias and are hypothized to reduce the risk. In total 64 different drugs were identified.

\begin{tabular}{|c|c|c|c|c|c|c|c|c|}
\hline Drug_ID & Drug name & Type & Mechanism_of_action & Action type & Disease_ID & Disease name_originally used for Phase & Status & Source \\
\hline CHEMBL2107842 & ALBINTERFERON ALFA-2B & $\begin{array}{l}\text { Protein } \\
\end{array}$ & Interferon alpha/beta receptor agonist & Agonist & EFO_0003047 & hepatitis C virus it & 2 Completed & https://clinicaltrials.gov/search?id=\%222NCT00097435\%22 \\
\hline CHEM BL2107842 & ALBINTERFERON ALFA-2B & Protein & Interferon alpha/beta receptor agonist & Agonist & EFO_0004220 & chronic hepatitis Cl & 2 Completed & https:///linicaltrials.gov/search?id=\%22NCT00759200\%22,https://clinicaltrials.gov/sez \\
\hline CHEM BL2107842 & ALBINTEREERON ALFA-2B & Protein & Interferon alpha/beta receptor agonist & Agonist & EFO_0004239 & chronic hepatitis & 1 Terminated & https://clinicaltrials.gov/search?id=\%22N CT00964665\%22 \\
\hline CHEM BL2108252 & APOUZUMAB & Antibody & Human leukocyte antigen DR beta chain inhibitor & Inhibitor & EFO_0000095 & chronic lymphoc & 1 Terminated & https:///linicaltrials.gov/search?id=\%22NCT00022971\%22 \\
\hline CHEM BL2108252 & APOUZUMAB & Antibody & Human leukocyte antigen DR beta chain inhibitor & Inhibitor & EFO_0000403 & diffuse large B-cell lymphoma & 1 Terminated & 622 \\
\hline CHEMBL2108252 & APOUZUMAB & Antibody & Human leukocyte antigen DR beta chain inhibitor & Inhibitor & EFO_0000574 & lymphom & 1 Completed & 122 \\
\hline CHEMBL2108252 & APOUZUMAB & Antibody & Human leukocyte antigen DR beta chain inhibitor & Inhibitor & EFO' 1001469 & Mantle cell lymphoma & $1 \mathrm{Te}$ & $=622 \mathrm{NCTO0}$ \\
\hline 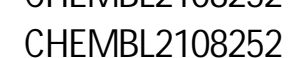 & APOUZUMAB & Antibody & $\begin{array}{l}\text { Human leukocyte anticen DR beta chain inhibitor } \\
\text { Hent }\end{array}$ & Inhibitor & MONDO 0018906 & follicular Iymphoma & 1 Terminated & https://clinicaltrials.ovo/search?id=\%22N CT00022971\%22 \\
\hline CHEM BL4297888 & APX-005M & Unknown & Tumor necrosis factor recepetor superfamily member 5 agonist & Aconist & $\begin{array}{lll}\text { EFO } 1000057 & \end{array}$ & rectum cancer & 2 Recruitinn & 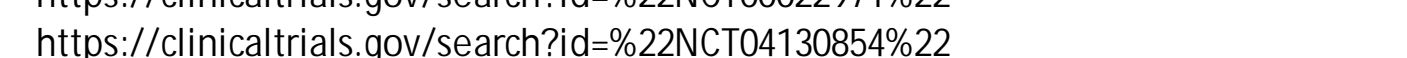 \\
\hline CHEM BL4297888 & APX-005M & Unknown & Tumor necrosis factor receptor superfamily member 5 agonist & Agonist & EFO' 1001968 & soft tissue sarcoma & 2 Recruitina & 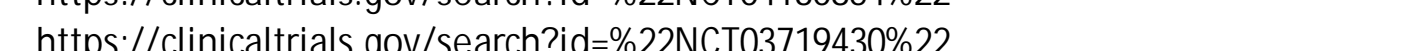 \\
\hline CHEM BL4297888 & APX-005M & Unknown & Tumor necrosis factor receptor superfamily member 5 agonist & Agonist & MON̄DO 0007576 & esophageal cancer & 2 Recruitina & 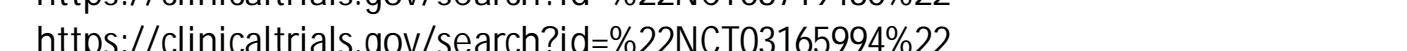 \\
\hline CHEM BL4297888 & APX-005M & Unknown & Tumor necrosis factor receptor superfamily member 5 agonist & Agonist & EFO 0000756 & $\begin{array}{l}\text { melanoma } \\
\text { ma }\end{array}$ & 1 Recruitina & 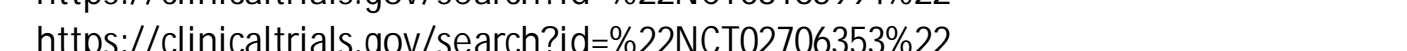 \\
\hline CHEM BL4297888 & APX-005M & Unknown & Tumor necrosis factor receptor superfamily member 5 agonist & Agonist & EFO'0002617 & metastatic melanoma & 1 Terminated & 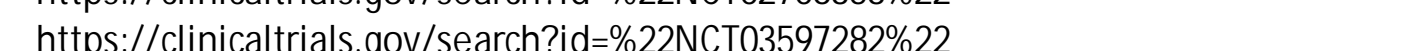 \\
\hline CHEM BL4297888 & APX-005M & Unknown & Tumor necrosis factor receptor superfamily member 5 agonist & Agonist & $\mathrm{EFO}^{-1} 1000044$ & pancreatic adenocarcinoma & 1 Active, not recruiting & httrs://clinicaltrials.ovo/search?id=1222N CT03214250\% 22 \\
\hline EMBL2109266 & AVE-9633 & Antibody & Myeloid cell surface antigen CD33 binding agent & Binding agent & MON̄DO 0004643 & myeloid leukemia & 1 Terminated & httrs://clinicaltrials.ovo/search?id=1222N CT00543972\%22 \\
\hline EMBL3989989 & AXICABTAGENECILOLEUCEL & Gene & B-lymphocyte antigen CD19 binding agent & Binding agent & EFO 00000616 & neoplasm & & http: //www whocc.no/atc ddd index/? code $=01 \times 1 \times 70$ \\
\hline CHEMBL3989989 & AXICABTAGENECILOLEUCEL & Gene & B-lymphocyte antigen CD19 binding agent & Binding agent & EFO- 0011057 & neurotoxicity & 2 Recruiting & https:// clinicaltrials.gov/search?id=\%22N CT04150913\%22 \\
\hline CHEMBL3989989 & AXICABTAGENECILOLEUCEL & Gene & B-lymphocyte antigen CD19 binding agent & Binding agent & $\mathrm{EFO}^{-1001469}$ & Mantle cell lymphoma & 2 Active, not recruiting & 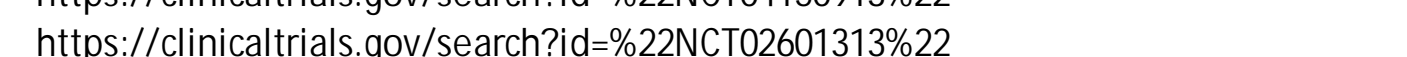 \\
\hline CHEMBL3989989 & AXICABTAGENECILOLEUCEL & Gene & B-lymphocyte antigen CD19 binding agent & Binding agent & EFO-0000096 & neoplasm of mature B-cells & 1 Active, not recruiting & 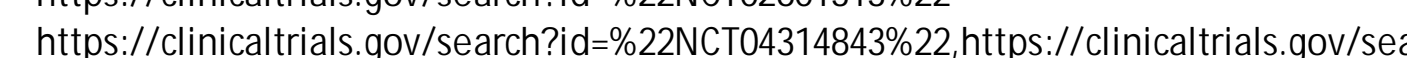 \\
\hline CHEMBL3989989 & AXICABTAGENECILOLEUCEL & Gene & agent & Binding agent & EFO-0000403 & diffuse large B-cell lymphoma & 1 Active, not recruiting & 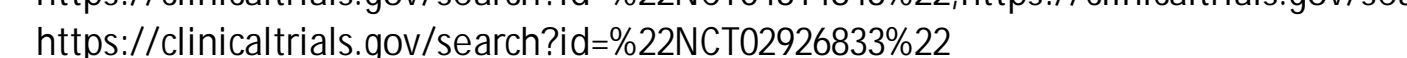 \\
\hline CHEMBL3989989 & AXICABTAGENECILOLEUCEL & Gene & te antigen CD19 binding agent & Binding agent & EFO-0000574 & lymphs & 1 Recruiting & https://clinicaltrials.ovo/search?id=\%22NCT04608487\% 22 \\
\hline CHEM BL3989989 & AXICABTAGENECILOLEUCEL & Gene & B-lymphocyte antigen CD19 binding agent & Binding agent & MONDO_0018906 & follicular lymphoma & 1 Active, not recruiting & https://clinicaltrials.gov/search?id=\%22NCT02348216\%22 \\
\hline CHEM BL3989989 & AXICABTAGENECILOLEUCEL & Gene & yte antigen CD19 binding agent & Bindinga & MONDO_0044889 & ohoma & 1 Active, not recr & https:///clinicaltrials.gov/search?id=0222N CT02348216\%22 \\
\hline CHEM BL4594618 & BREXUCABTAGENEAUTOLEUCEL & Gene & B-ly & $\mathrm{Bin}$ & EFO & Mar & 2 Active, not recr & https:///clinicaltrials.gov/search?id=\%22N CT02601313\%22 \\
\hline CHEM BL4594618 & BREXUCABTAGENE AUTOLEUCEL & Gene & ding agent & Bin & $\mathrm{EFO}_{-} \mathrm{O}$ & cyticleuke & 1 Active, not recruiting & https:///clinicaltrials.gov/search?id=\%22N CT03624036\%22 \\
\hline CHEM BL4594618 & BREXUCABTAGENE AUTOLEUCEL & Gene & yte antigen CD19 binding agent & g agent & EFO_0000220 & acute lymphoblastic leukemia & 1 Active, not recruiting & https://clinicaltrials.gov/search?id=ø22NCT02614066\%22 \\
\hline CHEMBL1201834 & CANAKINUMAB & Antibody & Interleukin-1 beta inhibitor & & EFO-0000540 & immune system disease & & http://www.whocc.no/atc_ddd_index/?code $=04 A C 08$ \\
\hline CHEMBL1201834 & CANAKINUMAB & Antibody & Interleukin-1 beta inhibitor & Inhibitor & Orphanet_208650 & Cryopyrin-associated periodic syndron & & http://dailymed.nlm.nih.göv/dāilymed/druglnfo. ffm?setid=7d271f3b-e4f9-4d80- \\
\hline CHEM BL1201834 & CANAKINUMAB & Antibody & Interleukin-1 beta inhibitor & Inhibitor & EFO_0003106 & pneumonia & 3 Completed & https://clinicaltrials.gov/search?id=\%22N CT04362813\%22 \\
\hline
\end{tabular}


atherosclerosis

Itps://Clinicaltrials.gov/search?id $=222 \mathrm{NCT} 01327846 \% 22$ $\begin{array}{ll}\text { Antibody } & \text { Interleukin-1 betainhibitor } \\ \text { Antibody } & \text { Interleukin-1 betainhibitor }\end{array}$ Antibody $\begin{array}{ll}\text { Antibody } & \text { Interleukin-1 beta inhibitor } \\ \text { Antibody Interleukin-1 beta inhibitor }\end{array}$ Antibody Interleukin-1 beta inhibitor Antibody Interleukin-1 beta inhibitor $\begin{array}{ll}\text { Antibody } & \text { Interleukin-1 beta inhibitor } \\ \text { Antibody } & \text { Interleukin-1 beta inhibitor }\end{array}$ Antibody Interleukin-1 betainhibitor Antibody Interleukin-1 betainhibitor Antibody Interleukin-1 betainhibitor
Antibody Interleukin-1 beta inhibitor Antibody Interleur Antibody Interleukin-1 betainhibitor Anlibody Interleukin-1 betainhibitor

systemic juvenile idiopathic arthritis
CINCA syndrome

Orphanet_1451

EFO-0000275

EFO-0001071

EFO-0001359

EFO-0002506

EFO_-0003780
EFO 0004214

EFO_0004246

EFO-0004265

EFO-O0004274

EFO -0007135

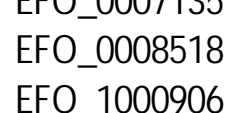

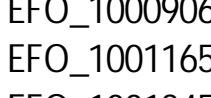

EFO_100135

HP 0000999
MONDO 0100000

Orphanet__32

orphanet_-230025

(F) 0000

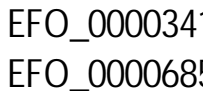

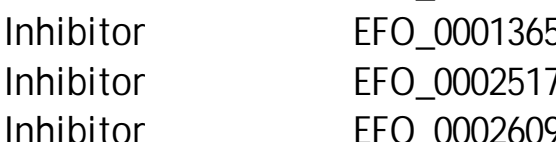
pulmonary sarcoidosis
atrial fibrillation

melanoma

type I diabetes mellitus

osteoarthritis

Behcet's syndrome mucocutaneous Iymph node syndrom gout

adult-onset Still's disease
polymyalgia rireumatica

shnitzler syndrom

Mepattisis, Alcoholic
Podedrma

Pyoderma
COVID-19

sickle cell anemia

Mevalonate kinase deficiency

amilial Mediterranean fever

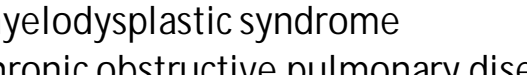

rheumatoid arthritis

pancreatic ductal adenocarcinom
juvenile idiopathicarthritis

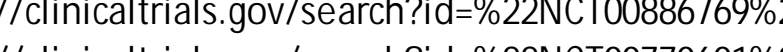

https:///linicaltrials.gov/ search?id=\%22NCTO2888000\%22 22

d= $=222 N C T 03484923 \% 22$

thtps:///linicaltrials.gov/search?id=\%22NCT04789681\%22

https://clinicaltrials.gov/search?id=\%22NCT00947427\%22

https:// clinicaltrials.gov/search?id=\%22NCT01160822\%22

https://linicaltrials gov/searchide

https://clinicaltrials.gov/search?id=\%22N CTO2980263\%22

https:// clinicaltrials.gov/search?id=\%22NCT01731990\%22

https:// clinicaltrials.gov/ search?id=2022NCT00819585\%22

https//dinicaltrials gov/searchide=222NCT02301203\%22

https:///linicaltrials.gov/ search?id=\%22NCT01364389\%22

https:// clinicaltrials.gov/search?id=\%22NCT01250171\%22

https://clinicaltrials.gov/search?id=2022NCT01390350\%22, https://clinicaltrials.gov/sea

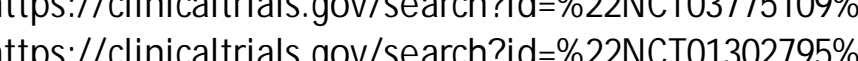

https://dinicaltrials.gov/searchidd=0222NCT04365153\%22

https://clinicaltrials.gov/search?id= $=222 \mathrm{NCTO2961218 \% 22}$

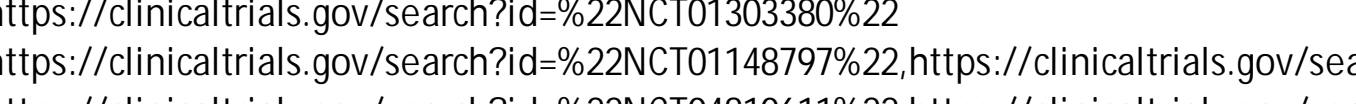

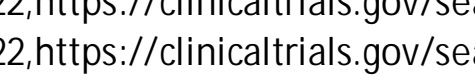

https:// clinicaltrials.gov/search?id=e222N CT00581945\%22

https://clinicaltrials.gov/search?id=\%22NCT00503022\%22

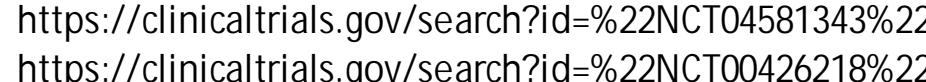




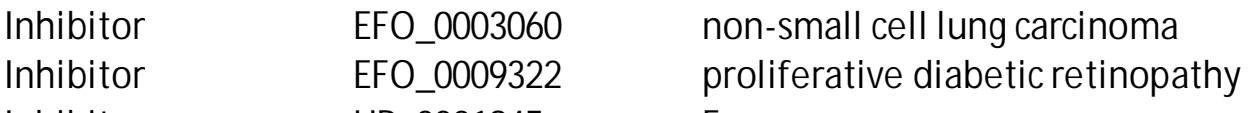

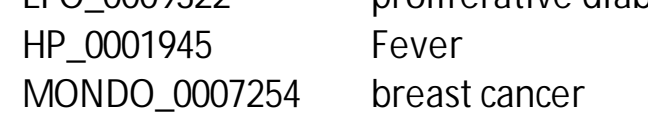

Inhibitor
Inhibitor

Inhibitor

EFO-000068

breast taner
renal cel carcinom
and

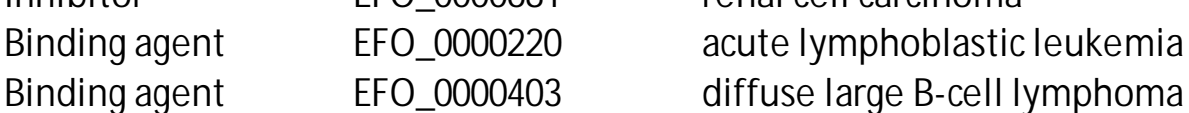

Bindingagent

Inhibitor
Inhibitor

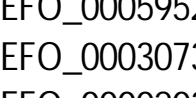

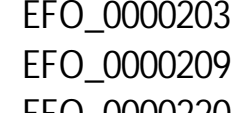

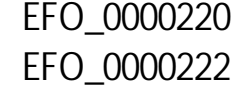

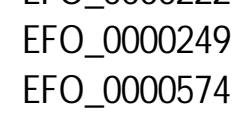

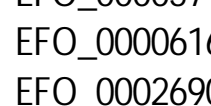

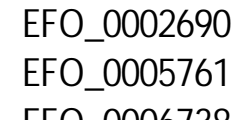

EFO-0006738
EFO-0007160

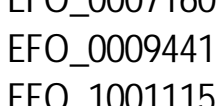

EFO-100115
EFO-1001469
EO

EFO-1001875

EFO-1001951

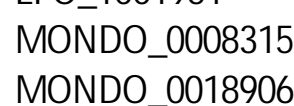

EFO -00000095

diffuse large B-cell lymphor
non-Hodgkins lymphoma

csymptomatic myeloma

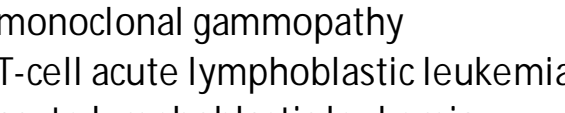

ante myeloid leukemia
andich

Alzheimer's disease

Iymphoma

systemicllupus erythematosus

nachtoma

auttimmune thrombocotopenic purpu
Waldenstrom macroglobulinemia
foll

Waldenstrom macco
PoEMS syndrome

amyloidosis

colorectal carcino
prostate cancer
follicular lympho

Inhibitor

Inhibitor
Inhititor
Inhibitor

Inhibitior
Inhibitor
Inhibitor

EFo 0000019

chronic lymphocytic leukemia

Hodgkins Iymphoma

myelodysplastic syndrome
breast adenocarcinoma
cancer

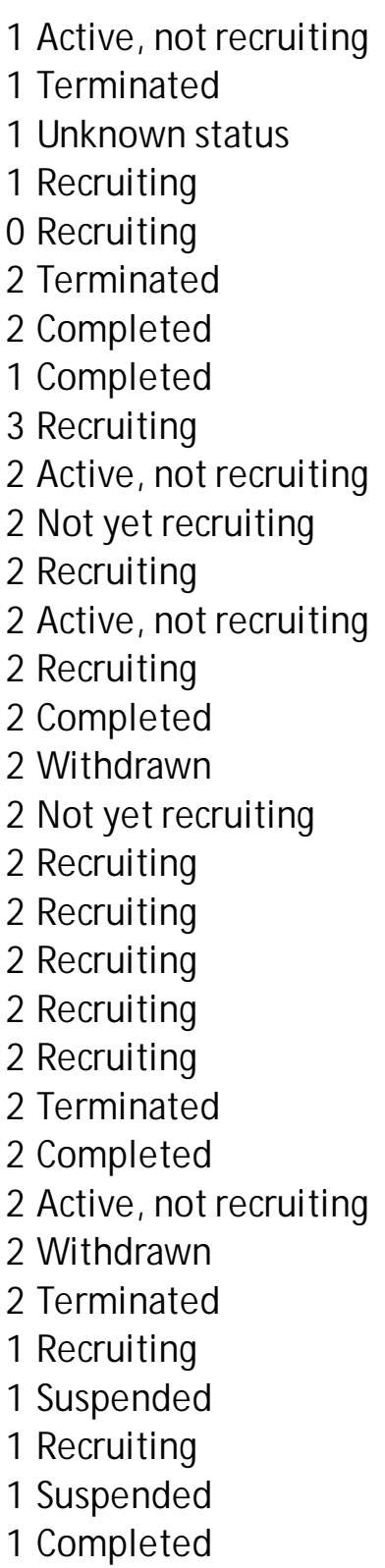

https://clinicaltrials.gov/search?id=\%22NCT03064854\%22 https:// clinicaltrials.gov/search?id=\%22NCT02775994\%22 hittps://clinicaltrials.gov/search?id=2222NCT03742349\% 22 https://dinicaltrials gov/searchid-et22NCT0140179\%22 https://clinicaltrials.gov/search?id=\%22NCT01472887\%22, https:///linicaltrials.gov/sea https://clinicaltrials.gov/search?id=\%22NCT00796731\%22, https:///linicaltrials.gov/sea https:// clinicaltrials.gov/ search?id=\%22NCT03937635\%22 https:// dinicaltrials.gov/search?id $=222 \mathrm{NCT} 03236428 \% 22$ https.// / Cinicaltrials.gov/ search?id=\%22NCT03384654\%22 https://clinicaltrials.gov/search?id=2\%22NCT04070378\%22 https://clinicaltrials.og

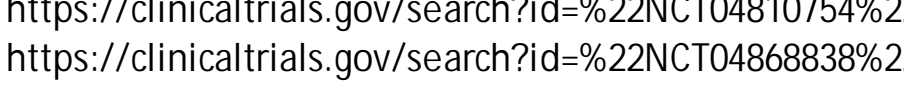
https:// clinicaltrials.gov/search?id=\%22NCT04166565\%22 https://clinicaltrials.gov/search?id=\%222NCT04703621\%22

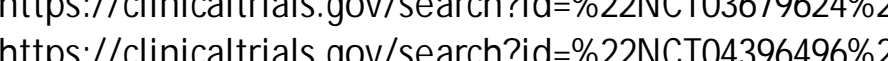
https://clinicaltrials.gov/search?id=\%22NCT02413489\% 22 https://clinicaltrials.gov/search?id=\%22NCT02816476\%22 https:///linicaltrials.gov/search?id=\%22NCT02060188\%22 https://clinicaltrials.gov/search?id=622N CTO303035357\%22

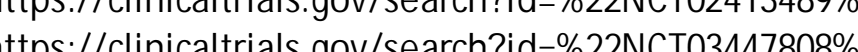

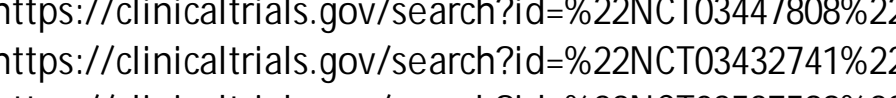
https:// clinicaltrials.gov/search?id=\%22NCT03537599\%22 https://clinicaltrials.gov/search?id=\%22NCT03432741\%22
https://clinicaltrials.gov/search?id=\%22NCT0309850\%22 


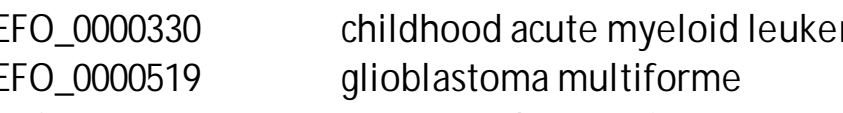

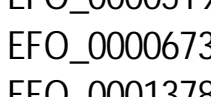

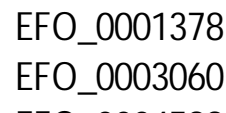

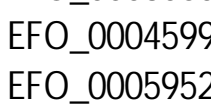

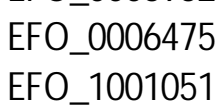

noñon-019946)

Orphanatet 8545
EFo 0000349

EF-0.0004033

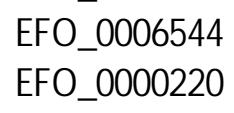

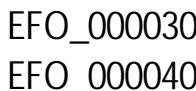

$E F 0-0000403$
EFO 1002169

MONDDO-0018900

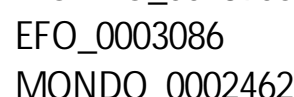

EFO 00001378
EF- 0004254

EFO 0000222

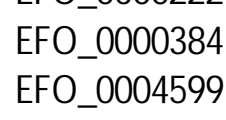

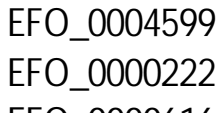

$E F O^{-0}-0000616$
$E F O-000220$

EF-00000224
EFO_0000095
EFO_0000198 acute myeloid
neoplasm

cute lymphoblastic leukemia

chronic lymphocytic leukemia

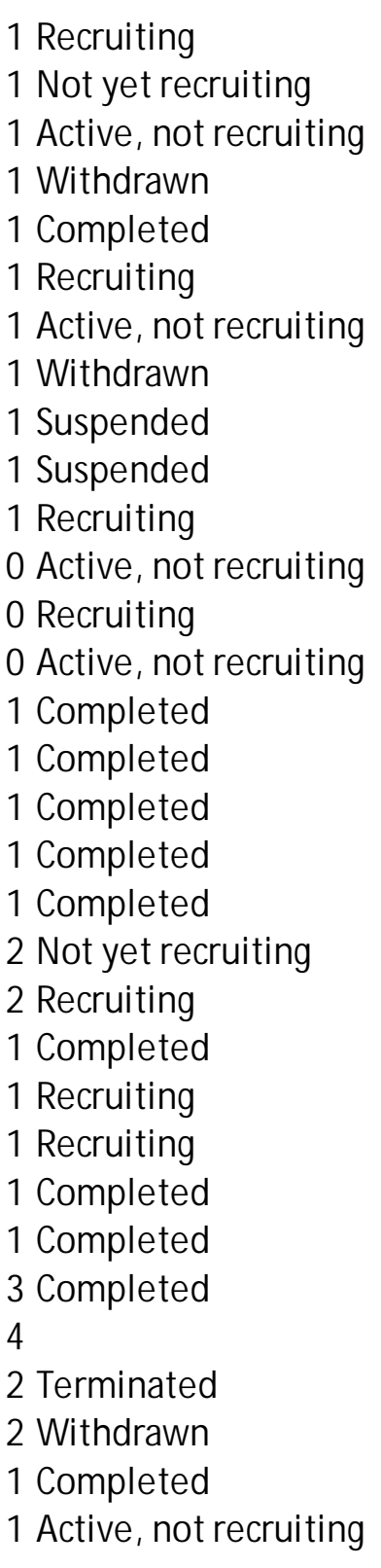

Ittps://clinicaltrials.gov/search?id=\%22NCT03537599\%22

https://clinicaltrials.gov/search?id=\%22NCT03177460\%22

ttps.//child

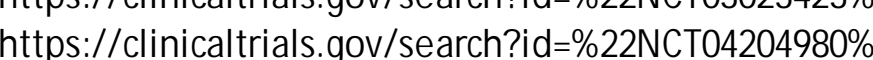

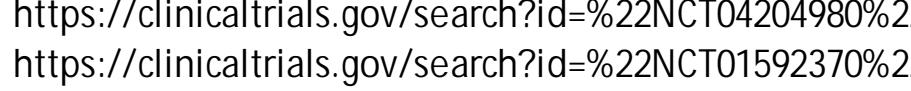

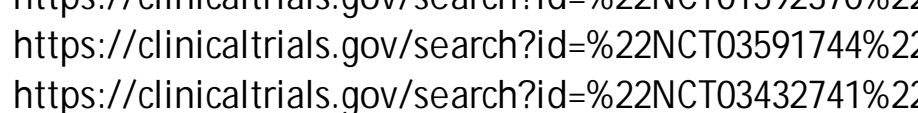

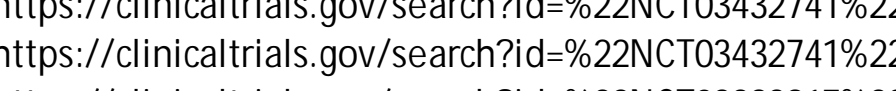

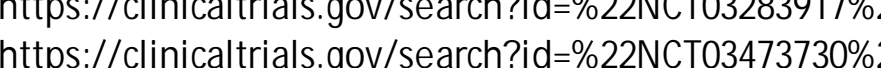

https://linicaltrials.gov/search?id=E222N CTO4139304\%22

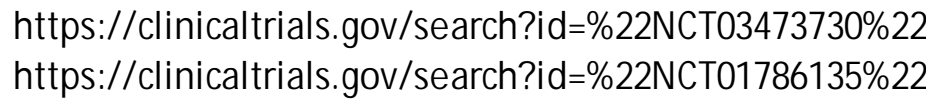

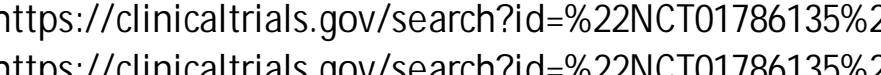

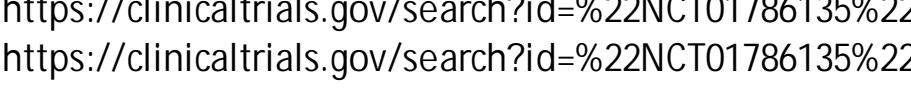

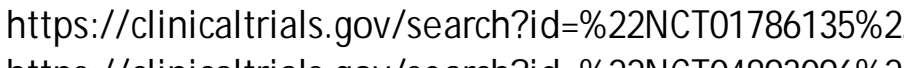

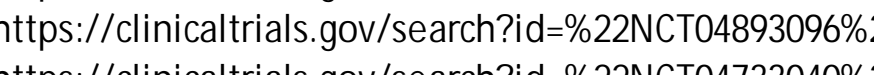

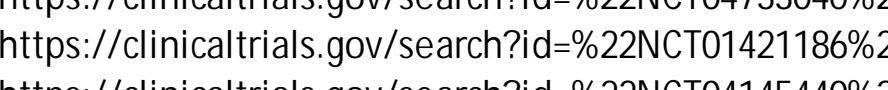

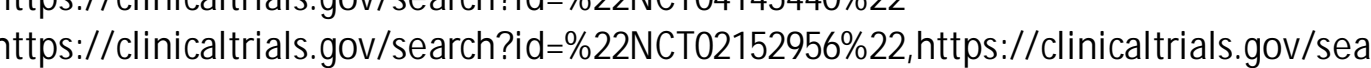

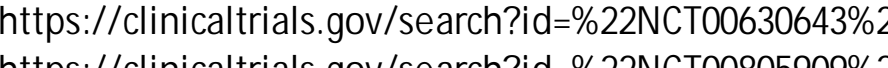

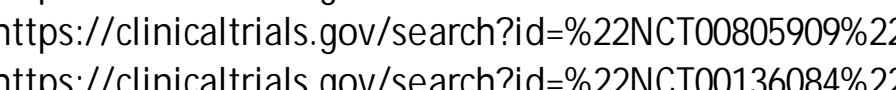

httro:/ wurw

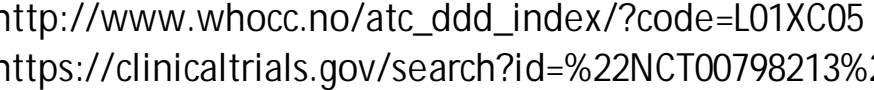

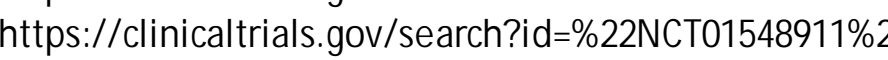

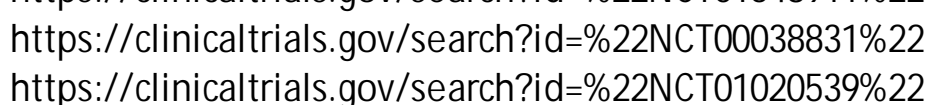




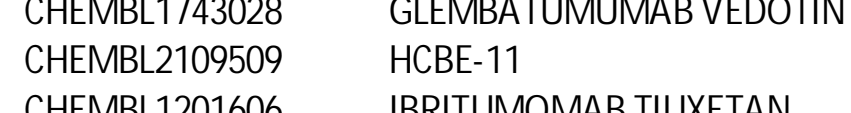

$\begin{array}{ll}\text { CHEMBLL21201006 } & \text { IBRTTUMOMAB TIUXEAN } \\ \text { BBRTUMOMAB TUXEEAN }\end{array}$

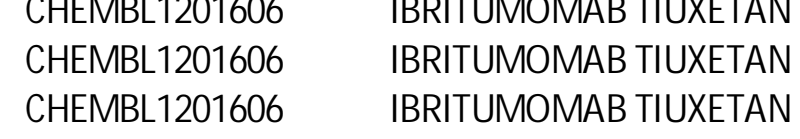

CHEMBLL201506 IBRTIUMOMAB TUXEEAN

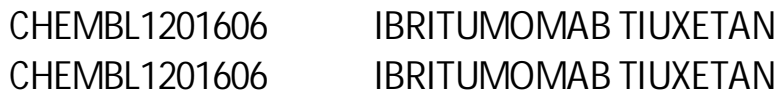

CHEMBLL201606

CHEEBBL120100606
CHEMBLL201006

leukemia
Juvenile Myelomonocytic Leukemia
therapy related accute myyloid leukem

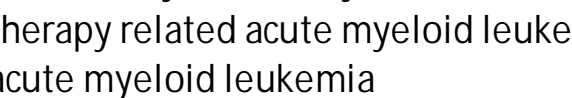

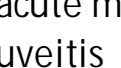
heumatoid arthritis type I liabetes mellitus
osteoarthritis acne Inner ear disease ypell diabetes mellitus Behcet's syndrome

scleritis
melanoma thentekin- - betan egative allosteric modulator Transmembrane e elycopoprotein NMB binding agent

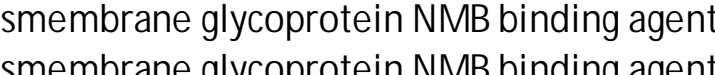
Tumor necrosis factor receptor superfamily member 3 ago B-Iymphocyte antigen CD20 binding agent
B-Iymphocyte antigen CD20 binding agant -lymphocyte antigen CD20 binding agent Hymphocte antigen CD20 binding agent B-lymphocyte antigen CD20 binding agent

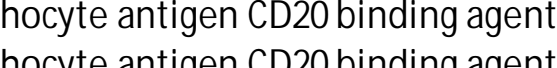

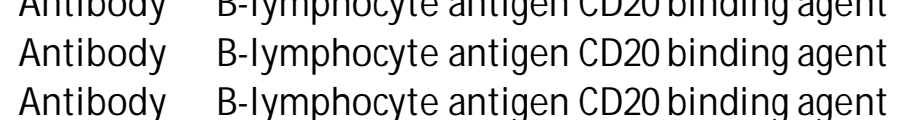

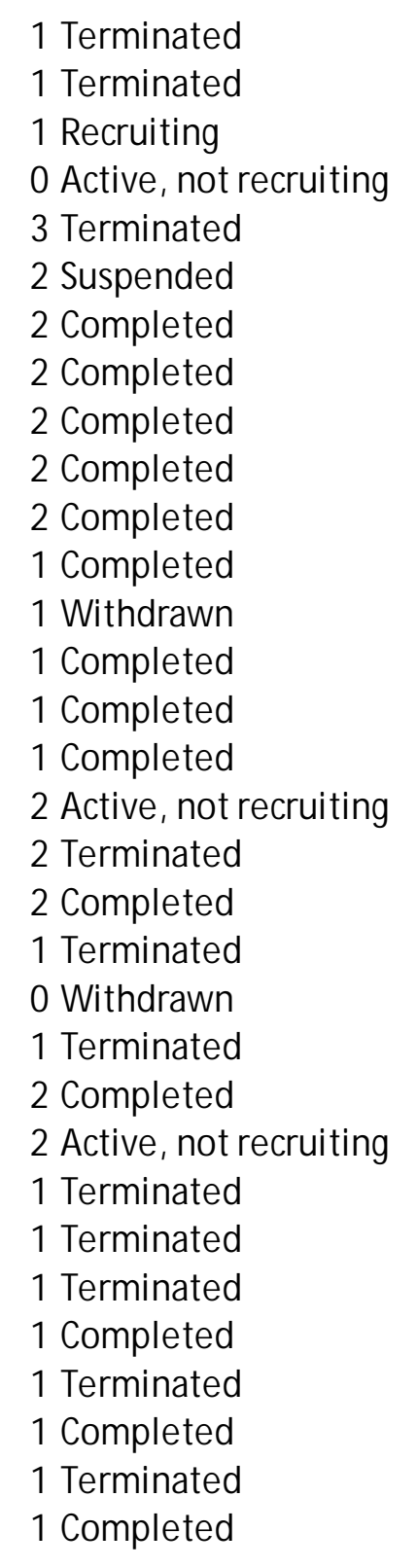

https:///linicaltrials.gov/search?id=\%22NCT00577694\%22

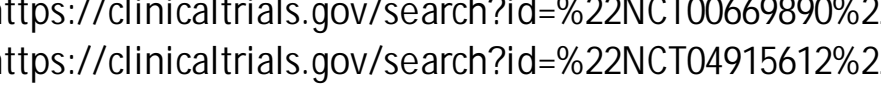
https:///linicaltrials. gov/search?id=2\%22N CT035689994\%22 /clinicaltrials.gov/sea https://clinicaltrials.gov/search?id=\%22NCT00777816\%22

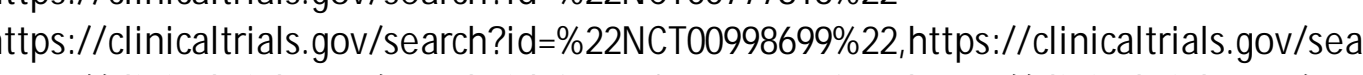

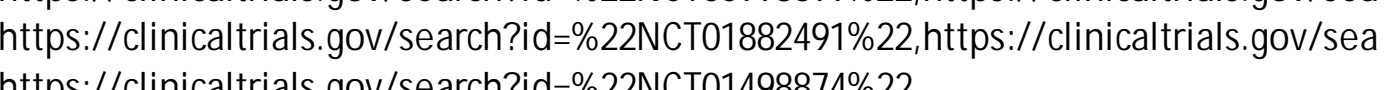

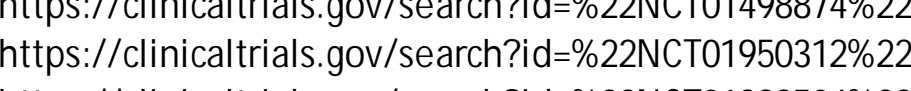

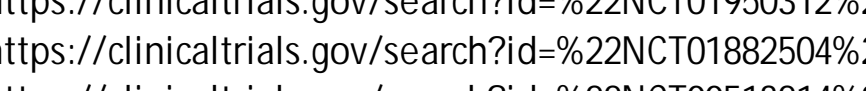

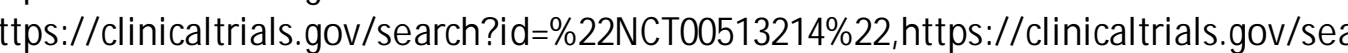

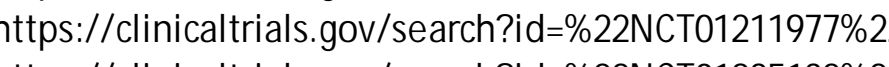

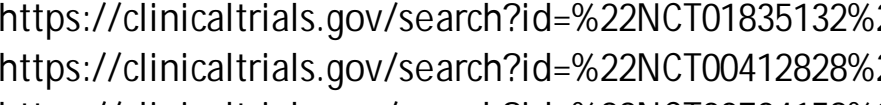

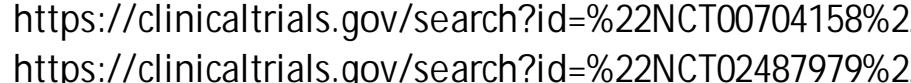

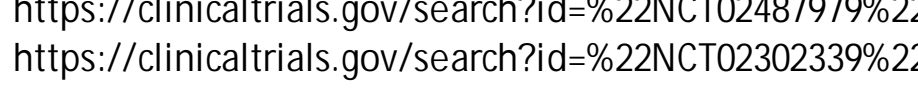

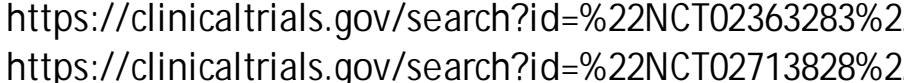

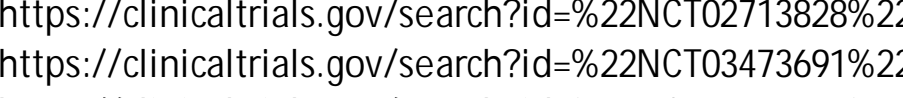

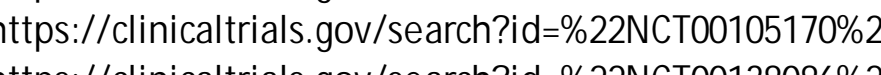

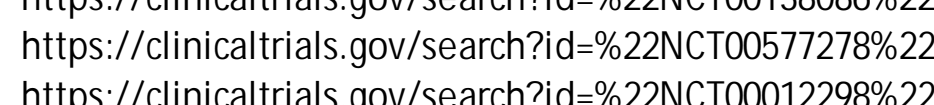

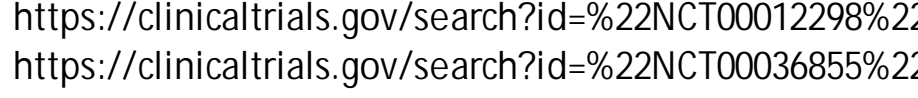

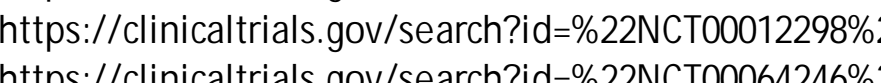

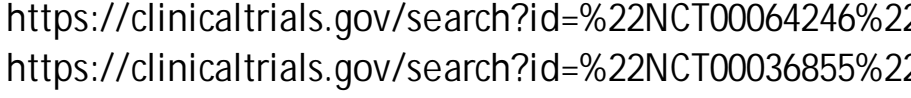
hittps://clinicaltrials.gov/search?id=\%22NCT00048737\%22

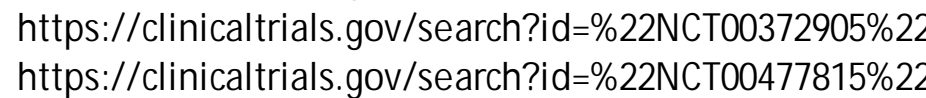


$\begin{array}{ll}\text { EFO_0000756 melanoma } \\ \text { EFO_0003047 } & \text { hepatitis Cvirus infection }\end{array}$

EFO- -000378

MFO-1001156

EFO_0000311

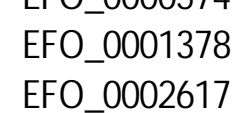

EFO -0002617
EFO 0004220

EFF-1000056

EF- -00000076

$E F O-0000681$
EFO 0000764

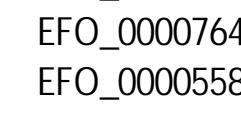

EFF-O0000574

EFF_-0007147

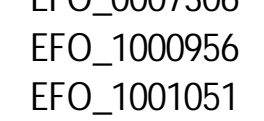

EFO-0000764

EFO-O000137
EFO_-O00142

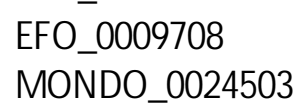

EFF_-0000349

EFO-000061
EFF- 0001688
EFO- 002618
Behcet's syndrome

retinal vasculitis
kidney cancer

cancer

lymphoma
multiple myeloma

metastatic melanoma

hairy cell leukemia

enal cell carcinoma

renal cell carcin
HIV infection
Kaposi's sarcom

Kaposi's sarcom
lymphoma

anogenital venereal wart

herpangina

mycosis fungoides

follicular lympho
Hivinection

nultiple myelo

multiple myeloma
cirrosis of liver

digestive system neuroendocrine neo

nic myelogenous leukemia

prostate adenocarcinom

renal cell carcinoma

human papilloma virus infection

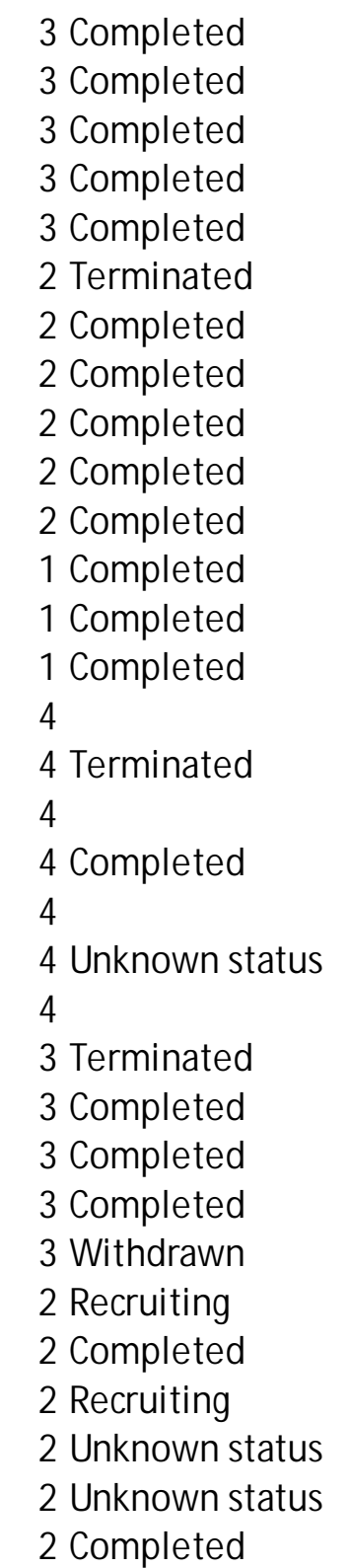

https:// clinicaltrials.gov/search?id=\%22NCT00204529\%22 https:// clinicaltrials.gov/search?id=0222N CT00167583\%22 https:///clinicaltrials.gov/search?id=\%22NCT00167583\%22 Attps://clincaltrials.gov/search?id=h22NCT02159482\%22 ttps:// clinicaltrials.gov/search?id=\%22NCT01404936\%22 https:// clinicaltrials.gov/search?id $=022 \mathrm{NCT} 00323505 \% 22$ https./// cinicaltrials.gov/search?id= $=222 \mathrm{NCT}(00308607 \% 22$ 畆 https:///linicaltrials.gov/search?id $=2222 \mathrm{NCT} 02763969 \% 2$ https://clinicaltrials.gov/ search?id=\%22NCT01513187\%22

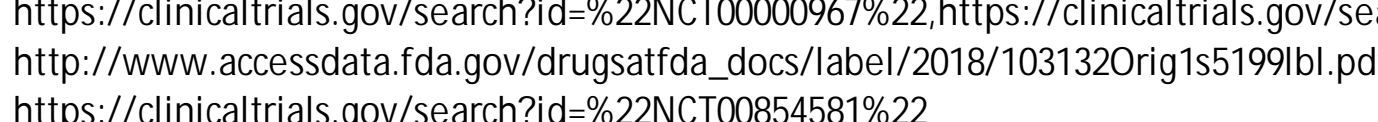
http:// www.accessdata.fda.gov/drugsatfda_docs//abel/2018/1031320rig1551991bl.pd http:/// www.accessdata.fda.gov/drugsatfda docs/label/2018/1031320rig155199|bl.pdf https:// clinicaltrials.gov/search?id=\%22NCT02323659\%22 2018/1031320rig1551991bl.pdf https://clinicaltrials.gov/search?id=\%222NCT00125814\%22 s://clinicaltrials.gov/sea https.//clinicaltrials.gov/search?ide\%22NCT00273247\%22

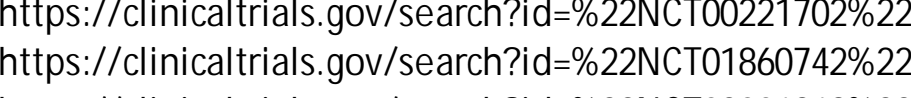
https://clinicaltrials.gov/search?id=\%22NCT00101114\%22,https:///linicaltrials.gov/sea https:/// clinicaltrials.gov/search?id=\%22N CT03899987\%22

https://clinicaltrials.gov/search?idd=/222NCT02801383\%22, https://clinicaltrials.gov/sea 
EFO_0004239 chronic hepatitis B virus infection

mucosal melanoma
Kidney cancer

MONDO_0002691 liver cancer

$\begin{array}{ll}\text { MONDO_0008315 } & \text { prostate cancer } \\ \text { MONDO_0019471 T-cell leukemia/lymphoma }\end{array}$

MONDO-001947
EFO 0000182

EFO-_0000311

EFO-0000389
EFO_-0000616

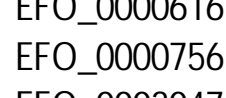

EFO-0003869

EFO-0004197

EFE -0004220
MONDO 2004558

MONDO-0008170

MONDO-004888

MONDODO007254

MONDD_-00100034

EFO -0000094

EFO_0003047

$E F F-0004220$
$E F-0007228$

EFO-_00031106

EFO-0007328

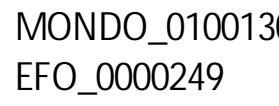

EFO-0000249 utaneous melanon neoplasm hepatitis Cvirusinfection epatitis B virus infection east carcinoma in situ varian cancer breast cancer

severe acute respiratory syndrome

epatitis C virus infection

chronic hepatitis Cvirus infection

prenmonia

hfluenza

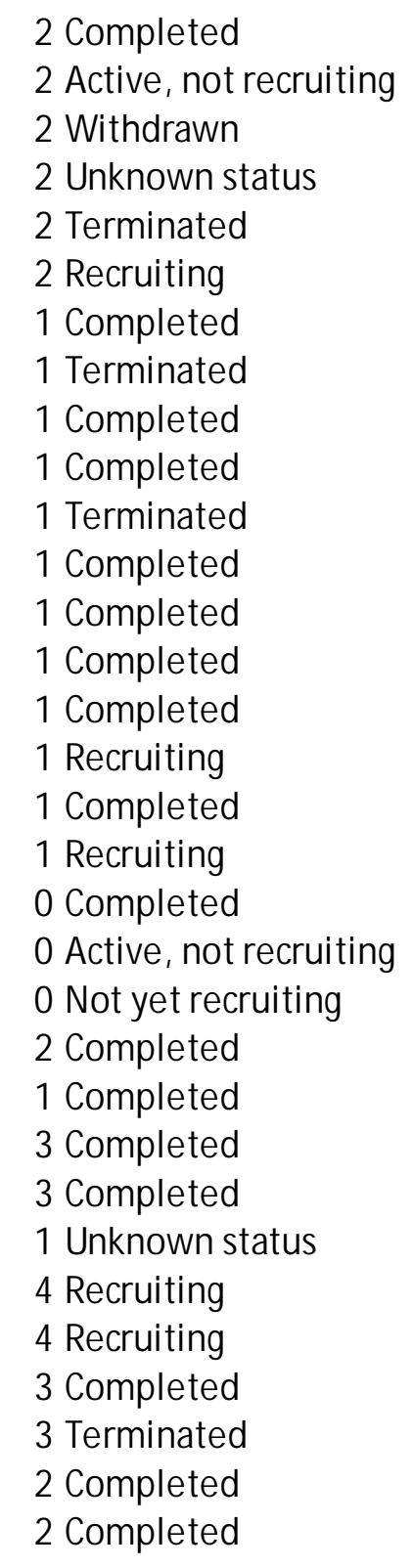

https://clinicaltrials.gov/search?id=\%22NCT00275938\%22

id $=\% 22$ NCT00293527\% 22 https:///linicaltrials.gov/search?id=\%222N CT00471484\% https://dinicaltrials gov/search?id-2022NCT02737046\%22 https:// clinicaltrials.gov/search?id=\%22NCT00234182\% 22 https:// clinicaltrials.gov/ search?id=\%22N CT00311558\%22

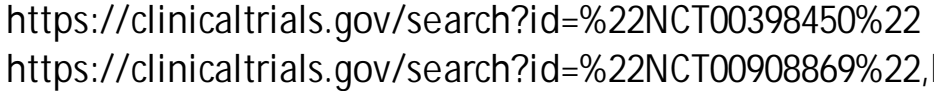

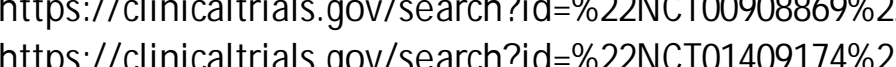
ps:// clinicaltrials.gov/sea poietic and lymphoid cell neor

dult tacute respiratory distress syndro

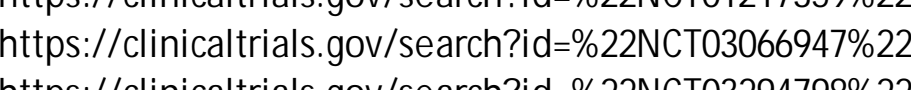

ttps:///clinicacaltrials. gov/search?ide\%22N NT01194037\%22

https://clinicaltrials.gov/sea

ittps://clinicaltrials.gov/search?id $=2222 \mathrm{NCT}$ T04081389\%22

https:///clinicaltrials. gov/search?id=2222NCT04379518\%22

https:// clinicaltrials.gov/search?id $=222 \mathrm{NCT02331706 \% 22}$

https://clinicaltrials.gov/search?id=\%22N CT03599453\%222

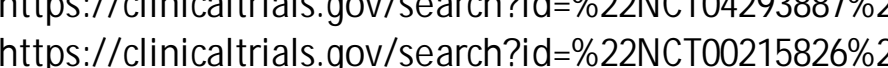

https://clinicaltrials.gov/searchidd=0222NCT000020780\%2

https://clinicaltrials.gov/search?id=\%22NCT00239252\%22

https://clinicaltrials.gov/search?Id=\%22NCT00162734\%22,https:// clinicaltrials.gov/sea

https://clinicaltrials.gov/search?id=\%22NCT01227798\%22

https://dinicaltrials gov/searchidef(22N CT02735707\%22

https:// clinicaltrials. gov/ search?id=\%22N CT002439860\%22

https://clinicaltrials.gov/search?id=\%22NCT02622724\%22

https://clinicaltrials.gov/ search?id=\%22NCT01075763\%22 
EFO_000341 chronic obstructive pulmonary disease

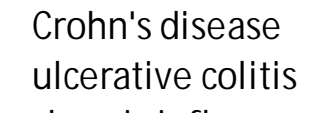

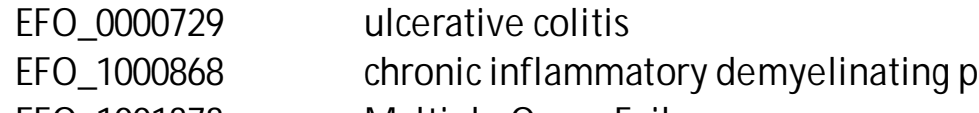

EFo 10001373 Multiple Organ Failure

$\begin{array}{ll}\text { MONDO } 0100096 & \text { COVID-19 } \\ \text { EFO } 0000712 & \text { stroke }\end{array}$

EFo-0003929 relapsing-remitting multiple sclerosis

EFO-_000388

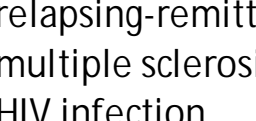

EFO-000318 Hiv infection

EFO 0003929 relapsing-remitting multiple sclerosis

MONDD_0100096 COVID-19

MONDO 0100116 Middle East respir

EFO-0000203

Middle East resp
nuttiple sclerosis

EFO-0000220

$E F O-0003073$
EFO-0005992

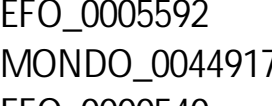

EFO 00000540
EFO 0000574

EFO_00006616

$E F=-0001378$
EFF -0000162

EFO-1001264

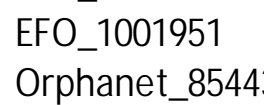

monoclonal gammopathy

acute myeloid leukemia

asymptomatic mye
T-cell lleukemia

T-cell leukemia
T-lymphoblasticlymphom

Tlymphoblastic lymphoma
immune system disease

neoplasm

neoplasm
multiple myeloma

Iymphoid neoplasm

Inhibititor
Inhibitor
this

Inhibititor
Bindingagent

Onding
other
Other
MONDO_0001475 neutropmphocytic leukem

neutropenia

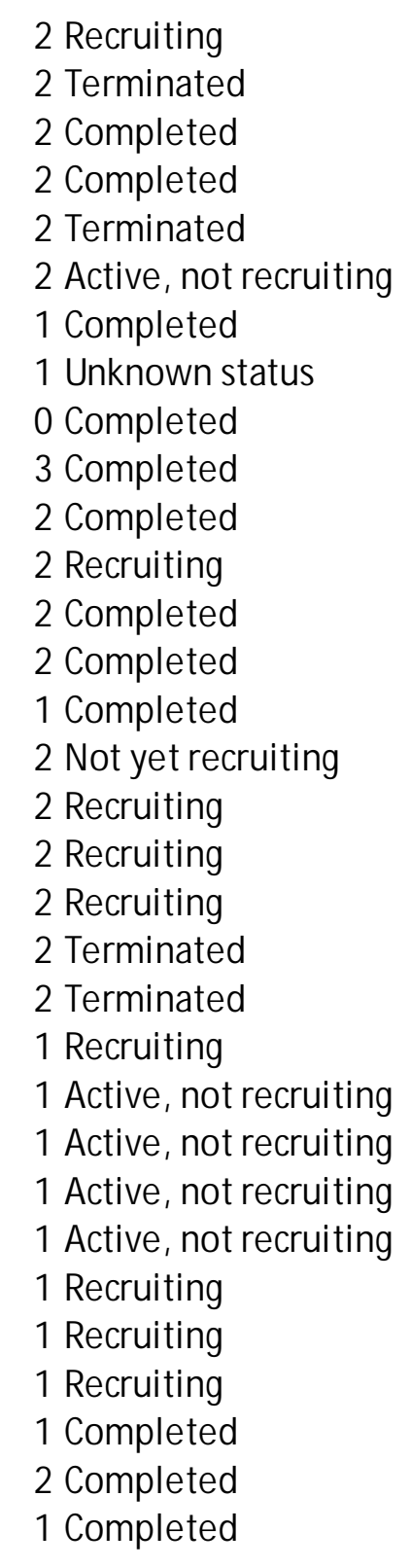

Ittps://clinicaltrials.gov/search?idd $=222 \mathrm{NCTO} 3570359 \% 222$

ps://clinicaltrials.gov/se

https:// clinicaltrials.gov/ search?id=2022NCT00099489\% 22

https://clinicaltrialss gov/searchiide/222NCT04385095\%22

https:// clinicaltrials.gov/ search?id=\%22N CT04385095\%22

https:// clinicaltrials.gov/search?id= $=222 N$ CTO2364986\%22
https://dinicaltrials.gov/search?id $=222 N$ CT00618527\%22

https:///clinicaltrials.s.gov/search?id=\%22NCT00002238\% 22

https://clinicaltrials.gov/search?id=\%22NCT00185250\%22

.

https:///clinicaltrials.gov/search?id=\%22NCT02845843\%22
https://clinicaltrials.gov/search?id=\%22N CT02474134\%22

ittps.//dinicutials gowseach

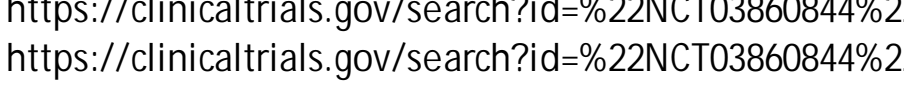

https://clinicaltrials.gov/search?id=2\%22NCT02960555\%22

https:///clinicaltrials.gov/search?id=\%22N CT02999633\%22

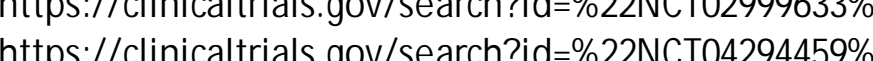

https:// clinicaltrials.gov/search?id=\%22N CT03769181\%22

https://clinicaltrials. gov/search?id=\%22N CT03637764\%22

https:// clinicaltrials.gov/search?!d=\%222NCTO2514668\%22, https:// clinicaltrials.gov/sea

https://clinicaltrials.gov/search?id=\%22NCT01084252\%22

https:// clinicaltrials.gov/search?id=0222N CT03555149\%22

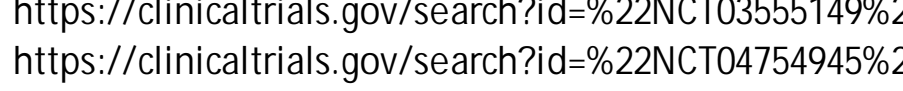

https://clinicaltrials.gov/search?id=\%22N CT01098188\% 22

https://clinicaltrials.gov/ search?id=\%22NCT00002800\% 22 
$\begin{array}{ll}\text { EFO_0000222 } & \text { acute myeloid leukemia } \\ \text { EFO_0000565 leukemia } & \end{array}$

EFO-0001378

EFO-100179

EFO_0005952

EFO-0000403

EFO_1001469

MONDO_0018906
EFO_1001938

MENDO 1001938

EFO_0000096

EFO-0000220

EFO-00004003
EF 01000789

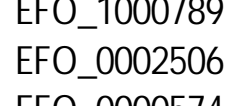

$E F O$
EFO-0000574

EF- 0000565

$E F F-0004251$
$E F 0$
$E F 000095$

EFO-0000095
EFO-000065

EFO-1000158

EF- 0007160

FFO-000137

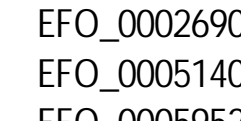

EFO-0005952
EFO__oocogs

EFO-0000095 Mantle cell lymphoma (d) Myasthenia gravis multiple myelom

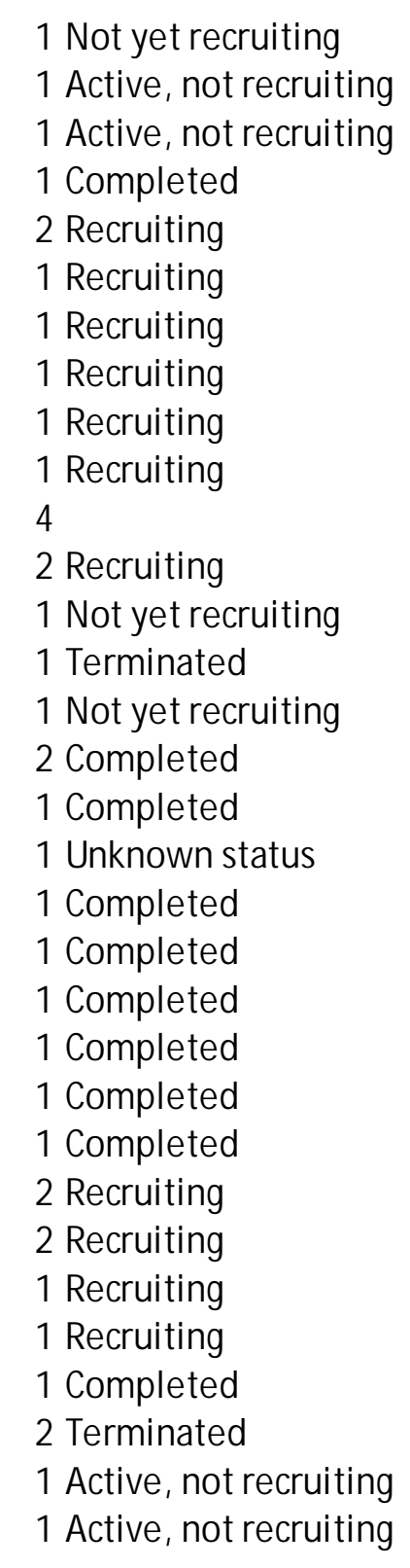

Attps:// clinicaltrials.sov/search?id=\%22NCT039323318\%22

ch?id=\%22N CT02998047\%22 https:// clinicaltrials.gov/ search?id=022NC C.00283114\%22 https:///linicaltrials. gov/search?id=\%222NCTO3331198\%22 https:// clinicaltrials.gov/search?id $=2222 \mathrm{NCTO} 2631044 \% 222$. Uffuse large B-cell lymphoma B-cell non-Hodgkins lymphoma neoplasm of mature B-cells acute lymphoblastic leukemia
diffuse large B-cell lymphoma

myelodysplastic syndrome myeloproliferative disorder hronic lymphocytic leukemia
rheumatoid arthritis Central Nervous System Neoplasm

attomimmune disease

chronic lymphocytic leukemia
https://clinicaltrials.gov/search?id=622NCT04699461\%22

https://clinicaltrials.gov/search?id=\%22NCT02669264\%22

https:// clinicaltrials.gov/search?id=\%22N CT04974996\%22

https://linicaltrials sov/search?ide-222NCT01668511\%22

https:///linicaltrials.gov/search?id=/222NCT00009776\%22,https:// clinicaltrials.gov/sea

ittps://clinicaltrials.gov/search?id=\%222NCT00014495\%22, https://clinicaltrials.gov/sea

ttps:///linicaltrials.gov/search?id=2222NCT00014495\%22, https:///clinicaltrials.gov/sea

(1)

https:// clinicaltrials.gov/search?id=\%22NCT00005813\%22

ttps:// clinicaltrials.gov/search?id=\%22NC104159805\%22

https:// clinicaltrials.gov/search?id=\%22NCT04278924\%22

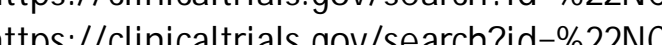

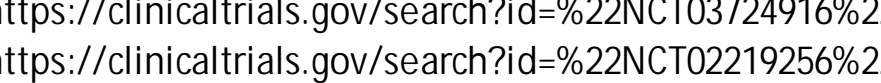

https:// clinicaltrials.gov/search?id=\%22NCT03645395\%22 2

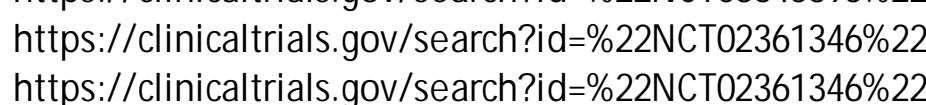


CHEM BL4297789 MT-3724

CHEMBLL1733048 OBN OBNUUZZUMAB

CHEM BL1743048 OBINUTUZUMAB

CHEMBL1743048 OBINUTUZUMAB

CHEMBL1743048 OBINUTUZUMAB

CHEMBL1743048 OBINUTUZUMAB

$\begin{array}{ll}\text { CHEMBL1743048 } & \text { OBINUTUZUMAB } \\ \text { CHEMBL1743048 } & \text { OBINUTUZUMAB }\end{array}$

CHEMBL1743048 OBINUTUZUMAB

CHEM BL1743048

CHEMBL1743048 OBINUTUZUMAB

(

CHEMBL1743048 OBINUTUZUMAB

CHEMBL1743048 OBINUTUZUMAB

CHEMBI774308 OBINUTUZUMAB

CHEMBL1773048 OBINUTUZUMAB

CHEMBLL7743048 OBINUTUZUMAB

CHEMBL1743048 OBINUTUZUMAB

CHEMBL1743048 OBINUTUZUMAB

CHEMBLL7743048 OBINUTUZUMAB

CHEMBL1743048

CHEM BL2109665

OCARATUZUMAB
OCREUZUMAB

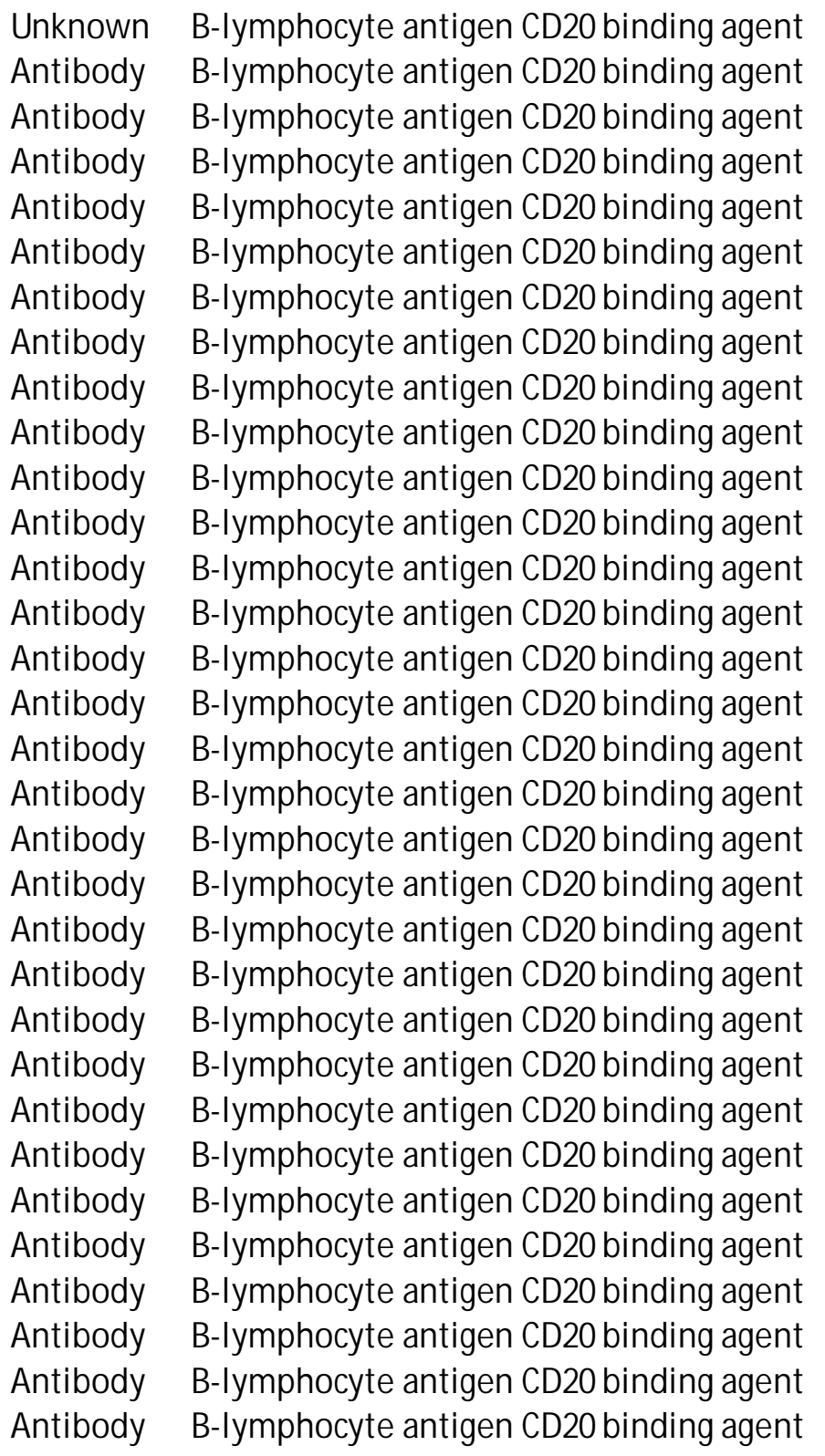

Binding agent
Binding agent
Binding agent
Binding agent
Binding agent
Binding agent
Binding agent
Binding agent
Binding agent
Binding agent
Binding agent
Binding agent
Binding agent
Binding agent
Binding agent
Binding agent
Binding agent
Binding agent
Binding agent
Binding agent
Binding agent
Binding agent
Binding agent
Binding agent
Binding agent
Binding agent
Binding agent
Binding agent
Binding agent
Binding agent
Binding agent
Binding agent

EFO_0004289 lymphoid leukemia

systemic lupus erythematosus

1 Withdrawn

3 Recruiting
2 Not yet recruiting

acute lymphoblastic leukemia

lupus nephritis
Waldenstrom macroglobulinemia

hairy cell leukemia

EFO-0005761

EFO_1000956

graft versus host disease

2 Active, not recruiting

2 Recruiting

2 Recruiting

EFO 0000096 neoplasm of mature B-cells

Hodgkins Iymphoma

EFO_0000304

breast adenocarcino
Burkitts lymphoma

EFF_-0000311

EFO_0000403

EFO-0000574

EFO-0000616

EFO_0005952
EFO-1001051

EFO_1001469

EFO-1001480

MONDO_0001023

MONDD_0002083
MONDO_0018906

MONDO 0019465

cancer

ffuse large - B-cell lymphoma

tmphoma

oplasm

chronickidney disease

mycosis fungoides

mantle cell lymphoma

colorectal carcinoma

filymphsyndrome

follicular lymphom mune system disease
https://clinicaltrials.gov/search?id=\%22NCT02556346\%22

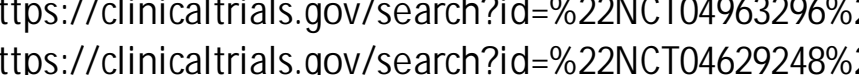

https:// clinicaltrials.s.gov/search?id = $=2222 \mathrm{~N}$ CTT04042020968\%22 22

htps://clinicaltrials gov/searchide=122NCT02550652\%22

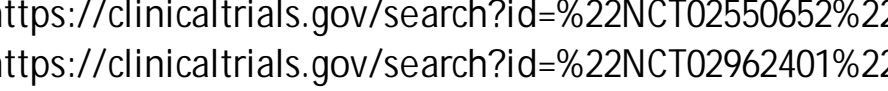

https:// clinicaltrials.gov/search?id=\%22N CTO3410875\%22

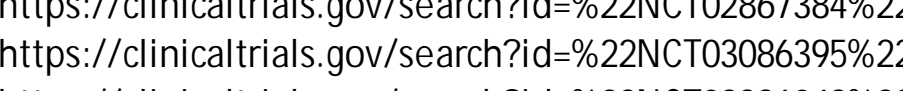

https://clinicaltrials.gov/search?id=\%22N CTO2336048\%22, https:// clinicaltrials.gov/sea

https:// clinicaltrials.gov/search?id=\%22NCT04313608\%22

https:// clinicaltrials.gov/search?id=\%22N CT03432741\%22

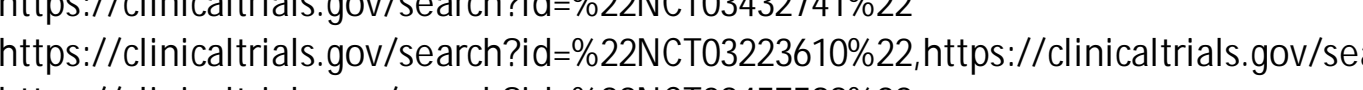

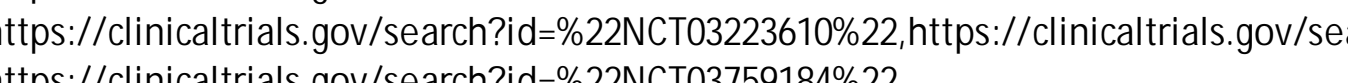

ths.//Cinicaltrials.gov/ search?id=/222NCT02729896\%22, https:// clinicaltrials.gov/sea

ttps:// clinicaltrials.gov/search?id=\%22NCT02324257\%22

hiths

https://clinicaltrials gow/searchide=622N CT03332741\%22

https:// clinicaltrials.gov/search?id=\%22NCTO2558816\%22

ttps:// clinicaltrials.gov/search?id=\%22NCT04826003\%22

https://clinicaltrials.gov/ search?id $=/ 22 \mathrm{~N}$ CT03866239\%22

https://dinicaltrials gov/search?id=2022N

https://clinicaltrials.gov/search?id=\%22N CTTO2631577\%22, https://clinicaltrials.gov/sea

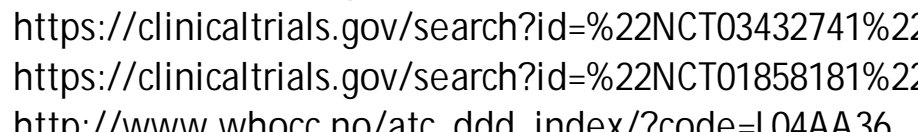


cancer
leukemia

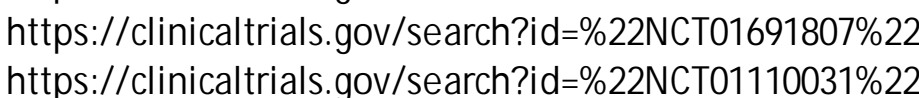




\begin{tabular}{|c|c|c|c|}
\hline & & & \\
\hline & (1) & & ng agent \\
\hline CHEM BLI201836 & DIMIN & & gg agent \\
\hline $\begin{array}{l}\text { CHEM BLI201836 } \\
\text { CHHM RI } 1201726\end{array}$ & A & & g agent \\
\hline $\begin{array}{l}\text { CHEM BLI201836 } \\
\text { CHHM RI } 1201726\end{array}$ & ATUMUMAB & Antib & ig agent \\
\hline $\begin{array}{l}\text { CHEM BLI201836 } \\
\text { CHHM RI } 1201736\end{array}$ & ATUMUMAB & Antibody & ig agent \\
\hline $\begin{array}{l}\text { CHEM BLI201836 } \\
\text { CHEMRI170160 }\end{array}$ & ATUMUMAB & Antibody & ig agent \\
\hline $\begin{array}{l}\text { CHEMBLL1201560 } \\
\text { CHEM BLI201560 }\end{array}$ & PEGINTERFERON ALFA-2A & $\begin{array}{l}\text { Protein } \\
\text { Drotain }\end{array}$ & onist \\
\hline $\begin{array}{l}\text { CHEMBLL1201560 } \\
\text { CHEM BLI201560 }\end{array}$ & PEGINTERFERON ALFA-2A & $\begin{array}{l}\text { Protein } \\
\text { Drmain }\end{array}$ & Interferon alpha/ beta recepto \\
\hline $\begin{array}{l}\text { CHEM BLL1201560 } \\
\text { CHEM BLL201560 }\end{array}$ & $\begin{array}{l}\text { PEGINTERFERON ALFA-2A } \\
\text { PPGIITRERPRN AIIA-AA }\end{array}$ & $\begin{array}{l}\text { Protein } \\
\text { Protein }\end{array}$ & Interferon alpha/ beta receptor agonist \\
\hline $\begin{array}{l}\text { CHEMBLL1201560 } \\
\text { CHEM BLL201560 }\end{array}$ & $\begin{array}{l}\text { PEGINTERFERON ALFA-2A } \\
\text { PEGGNTRERON AIFA-2A }\end{array}$ & $\begin{array}{l}\text { Protein } \\
\text { Protein }\end{array}$ & Interferon alpha/beta receptor agonist \\
\hline CHEM BL1201560 & $\begin{array}{l}\text { PEGGNTRRFRONON ALFA-2A } \\
\text { PEGINTERERON ALFA-2A }\end{array}$ & $\begin{array}{l}\text { Proteln } \\
\text { Protein }\end{array}$ & Interferon alpha//beta receptor agonist \\
\hline CHEM BLL201560 & $\begin{array}{l}\text { PEGINTERFERON ALFA-2A } \\
\text { PERTE }\end{array}$ & Protein & Interferon alpha/beta receptor agonist \\
\hline HEM BL1201560 & PEGINTEREERON ALFA-ZA & Protein & Interferon alpha/beta receptor agonist \\
\hline HEMBL1201560 & PEGINTERFERON ALFA-2A & Protein & Interferon alpha/beta receptor agonist \\
\hline HEMBL1201560 & PEGINTERFERON ALFA-2A & Protein & Interferon alpha/beta receptor agonist \\
\hline CHEMBLI201560 & PEGINTERFERON ALFA-2A & Protein & Interferon alpha/beta receptor agonist \\
\hline CHEMBLL2201560 & PEGINTERFERON ALFA-2A & Protein & Interferon alpha/beta receptor agonist \\
\hline CHEMBLL2201560 & PEGINTERFERON ALFA-2A & Protein & Interferon alpha/beta receptor agonist \\
\hline CHEMBLL2201560 & PEGINTERFERON ALFA-2A & Protein & Interferon alpha/beta receptor agonist \\
\hline CHEMBLL2201560 & PEGINTERFERON ALFA-2A & & Interferon alpha/beta receptor agonist \\
\hline CHEMBL1201 & PEGINTERFERON ALFA-2A & & Interferon alpha/beta receptor agonist \\
\hline CHEMBLL20 & PEGINTERFERON ALFA-2A & & Interferon alpha/beta receptor agonist \\
\hline CHE & INTE & & Interferon alpha/beta receptor agonist \\
\hline CHE & A & & Interfe \\
\hline CHE & A & & Interfe! \\
\hline CHE & A & & Interfe \\
\hline 0 & $A$ & & \\
\hline 0 & A & & \\
\hline 201560 & NITRERERON ALFA-2A & & \\
\hline BLI201560 & & Protein & gonist \\
\hline
\end{tabular}


EFO 0000764
EFO 0002229

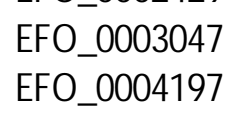

EFO-0004220

EFO-0004239
EFO-0007304

EFO- 0009260

MONDO-0044903

EFO_0000479

EFF_-_0000637

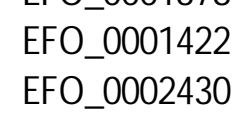

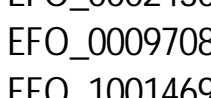

EFO-1001469

EFĒ_-0000326

EFO-0000389
EFO-0000555

EFO-0000574
EFO-000658

EFO_000065

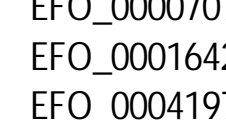

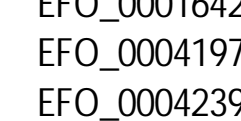

EFF-0004239

EFO-0004243

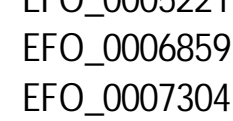

EFO-0007304
FFO-0009259
HIV infection
polycythemiaver

hepatitis Cvirus infection

epatitis B virus infection rronichepatitis Bvirus infection

Non-melanomas sin carcinom

$\begin{aligned} & \text { kidney cancer } \\ & \text { myelofibrosis }\end{aligned}$
s.

essential thrombocythemia

osteosarcoma
nultiple myeloma

dirrhosis of liver
primary myelofibrosis

metastasis

Mantle cell lymphoma

central nervous system can

cutaneous melanoma

leukemia

plexiform neurofibroma

lymphoid neoplasm

hepatitis B Bvirusinfinection

chronic hepeatitis
carcinoid tumor

cholangiocarcinoma
head and neck malignant neoplas

head and neck malingant neoplası
hepatitis D virus infection
skitsion

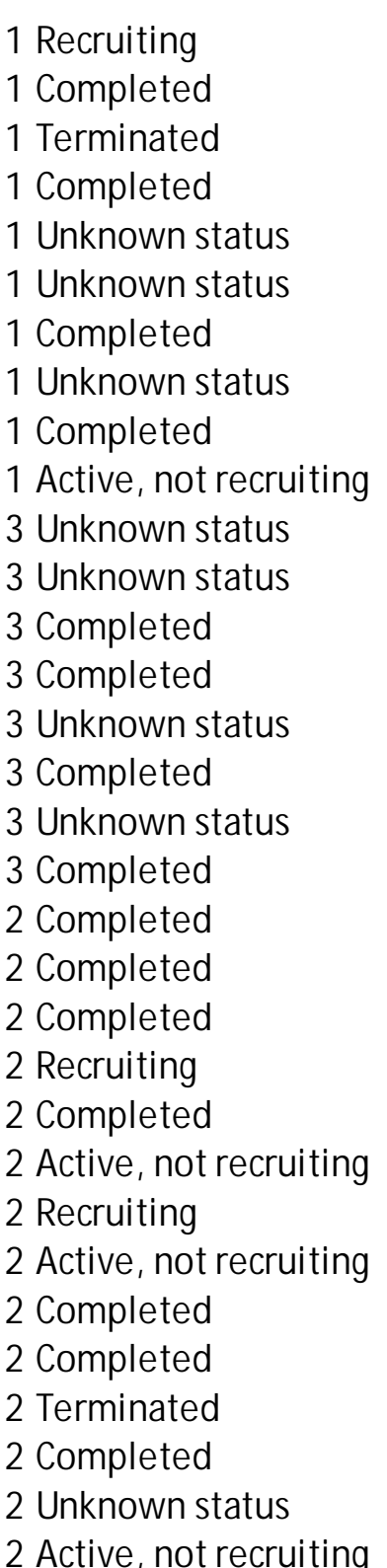

https://clinicaltrials.gov/search?id=\%22NCT02471430\%22 https:// clinicaltrials.gov/search?id=\%22N CT00277758\% 22 hittps://Chincialtrials.gov/ search?id= $=222 \mathrm{NCT} 03294798 \% 22$

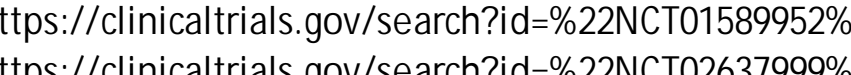
ttps:// clinicaltrials.gov/search?id=\%22NCT00423397\%22 https:// clinicaltrials.gov/search?id=\%22NCT00003542\%22 hittps:///linicaltrials.gov/search?id=\%222NCT027423234\%22 ttps://clinicaltrials.gov/search?id=\%22NCT00134030\%22 https://clinicaltrials.gov/search?id=\%22N CT00732641\%22

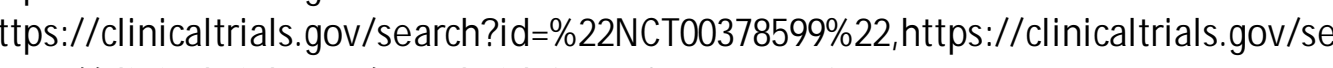
https://clinicaltrials.gov/search?id=\%222NCT01387763\%22 ttps///linicaltrils gov/searchid-ef22NCT00209209\%22 https://clinicaltrials.gov/search?id=\%22NCT00039871\%22 https:// clinicaltrials.gov/search?id=\%22NCT00047879\%22 https://cinicaltrials.gov/search?id=\%22N CT00049530\%22,https:///linicaltrials.gov/sea

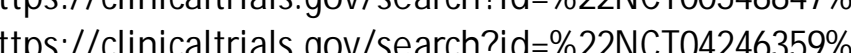
https:// clinicaltrials.gov/search?id=\%22NCT00396019\%22 https:///linicaltrials.gov/search?id=2222N CT02218164\%2

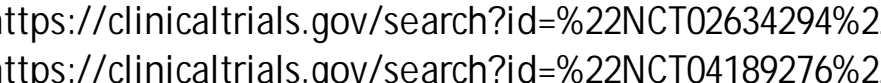

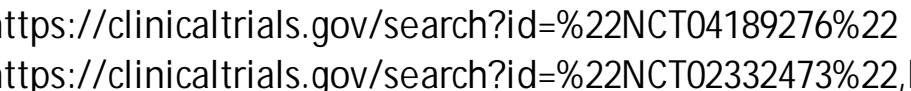
tps://clinicaltrials.gov/sea https:// / clinicaltrials.gov/search?id=\%222NCT00055809\%22 https://clinicaltrials.gov/search?id=\%22N CT00276523\%22

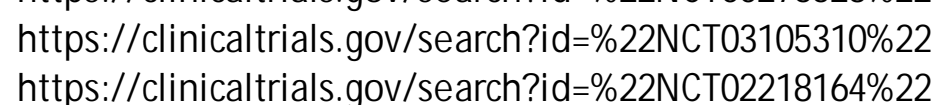


EFo-1000209
EFo-1000003

MoNDo-0002979 cenvical cancer

$\begin{array}{ll}\text { MONDO-0016691 } & \text { pilocytic astrocytoma } \\ \text { MONDD-018079 } & \text { thymic epithelial neoplasm }\end{array}$

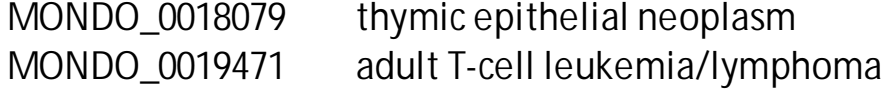

$\begin{array}{ll}\text { MONDD_0019471 } & \text { adult T-cer } \\ \text { MONDO_0100096 } & \text { COVID-19 }\end{array}$

Neurofibromatosis type 1

Orphanet_636

EFF-_0000182

EFO-0000339

EFO-0000616

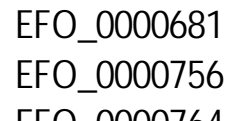

(F)-000076

EFO-0000765
EFO-0002429

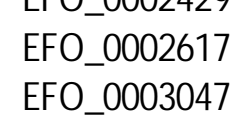

EFF-0004220

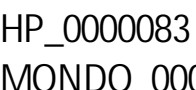

MONDO-0002158

MNDD_-0002367

MONDO-000817

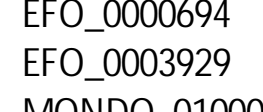

EFO 0003885

HP
HF- 000000033
FF -003929 neoplasm melanoma AIDS AIDS epatitis Cvirus infection Crirusinfection Renal insufficiency
peritoneum cancer

allopian tube cancer

kidney cancer
ovarian cancer relapsing-rerent
Covid-19

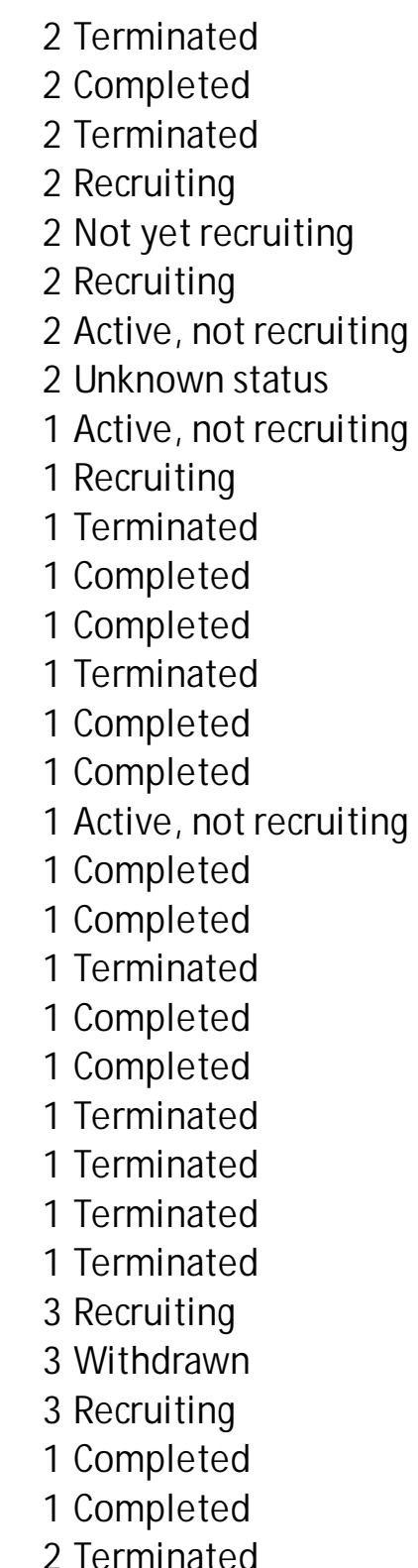

ttps://clinicaltrials.gov/search?id=222NCT01964300\%22 https:// clinicaltrials.gov/search?id=222N CT00138151\%22 hittps:I/ / cinicacaltrials.gov/search?id=\%222NCT023343224\%22 https://dinicaltrials.gov/searchidid-0222NCT02737046\%22 https:///linicaltrials.gov/search?id=\%222N CT04480138\% 222 https:///linicaltrials.gov/search?id=\%22NCT00846430\%22
https://clinicaltrials.gov/search?id=\%22NCT03588715\%22 https:// clinicaltrials.gov/search?id=\%22NCT0358875\%22

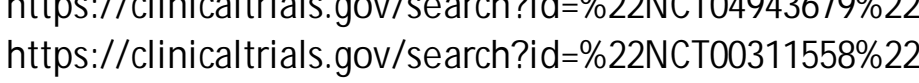

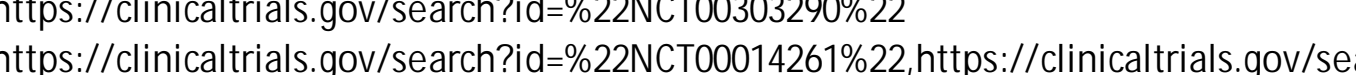
Attps:// clinicaltrials.gov/search?id $=222$ NCT00522249\%22, .htps://clinicaltrials.gov/sea https://clinicaltrials.gov/search?id=\%22NCT01496807/22,

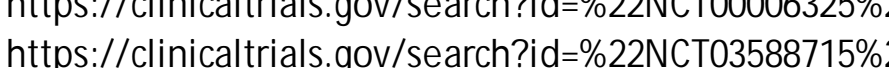
https:// clinicaltrials.gov/search?id=\%22NCT01193699\%22 https:// clinicaltrials.gov/search?id= $=222 \mathrm{NCT02379195 \% 22}$ https:// clinicaltrials. gov/search?idd=222N NT01701063\%22

severe acute respiratory syndrome

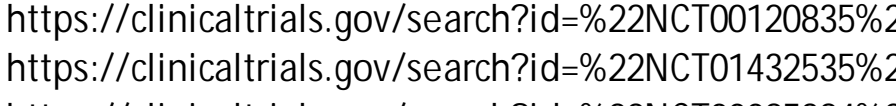
Hps://clinicaltrials.gov/sea https://clinicaltrials.gov/search?id=\%22NCT00085384\%22

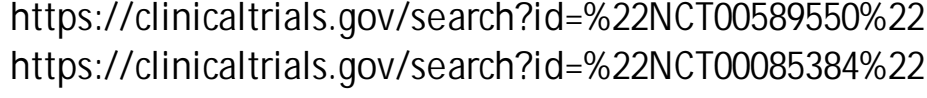
https:///linicatis https:// clinicaltrials. gov/s search?id=\%22N CT0404727424\%22 https:// clinicaltrials.gov/search?id=\%22NCT02269930\%22 https:///linicaltrials.gov/search?id=\%222NCT01119781\%22
https://clinicaltrials.gov/search?id=\%22NCT01963611\%22 


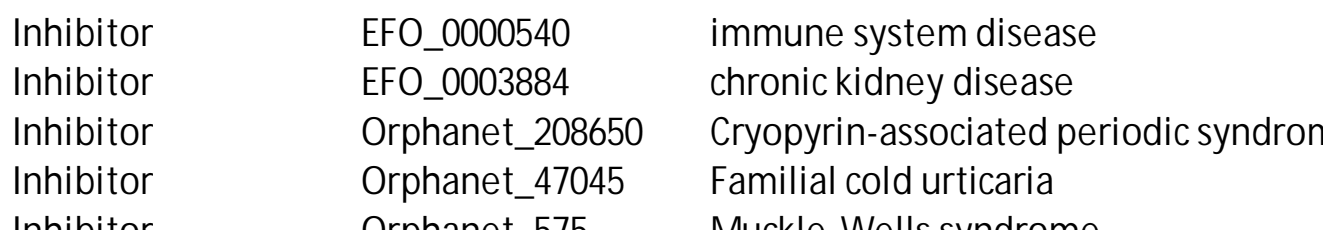

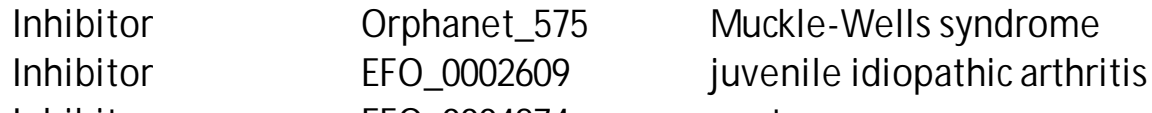

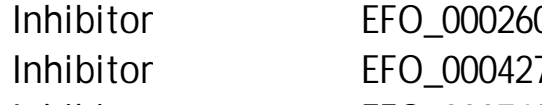

Inhibitior
Inhibitor

Inhibitor

Innibitor
Inhibitor

Inhibititor
Inhibitor
Inhibitor

Inhibitor
Inhibitor

Inhibititor
Innhititor
Inhibitor

Inhibitor
Binding agent
Binding agent

Binding agent
Binding agen
Binding agent

Bindingagen
Bindingagen
Bindingagent

Binding agent
Binding gagent
Binding gaent

Binding agent
Binding agent

Bindingagent
Binding agent

Bininingagent
Bindingagent

Binding agent
Bindingagent
Binding agent

Bininingagent
Bindingagent
Bindingagent

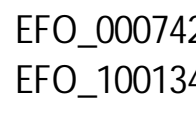

EFO-1001881

Orphanet_210115

EFO_0000404

EFO__0001359

EFO-1001999
EFO 0004238

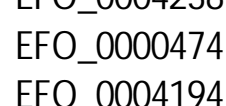

HP_-0000083

EFE-00003929

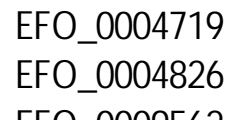

HP-0000093

MONDO_O0004

EFO 0000255

配0000389

EFO-0000540
EFO 0000557

$\mathrm{EF}-00000717$
EFF-0000729
EFO-000765

EFO-0000729
EF- 0000765

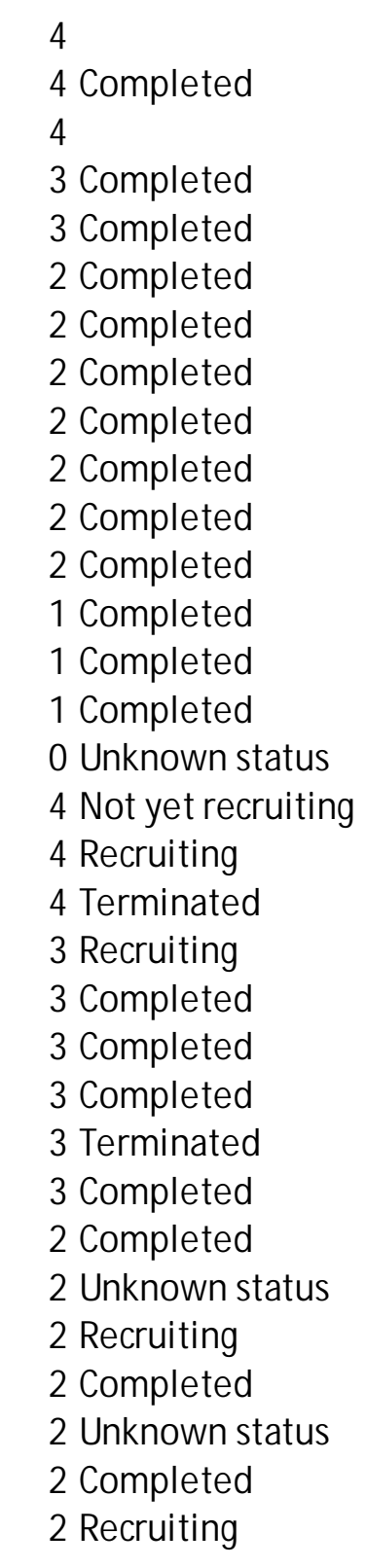

http://www.whocc.no/atc_ddd_index/? code=L04AC04

99-e848-432a-bac1-

https:// clinicaltrials.gov/search?id=\%22NCT00288704\% 22

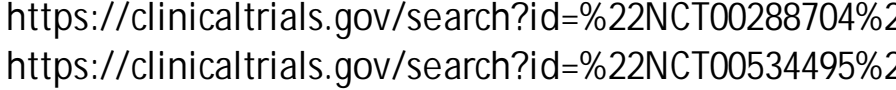

https:// clinicaltrials.gov/search?id=\%222N CT00534495\%22

https: // clinicaltrials.gov/search?id= $=222 N$ CTO3980522\%22
https://clinicaltrials.gov/ search?id=\%22NCT01903798\%22

https:///linicaltrials.gov/search?idd\%222NCT02171416\%22

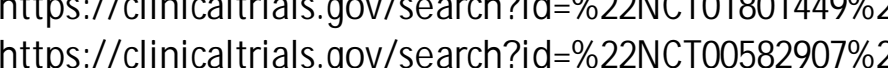

https:// clinicaltrials.gov/search?id=\%22N CT01538719\%22

https:///clinicaltrials.gov/search?id=\%22NCT00962026\%22
https://clinicaltrials.gov/s search?id=\%22NCT01803321\%22

https://clinicaltrials.gov/search?id=\%22NCT02828033\%22

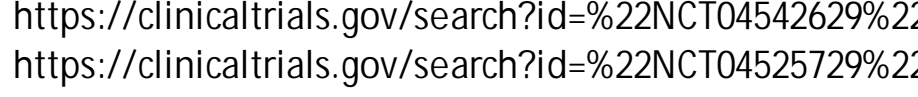

https://clinicaltrials.gov/search?id=\%22NCT01312064\%22

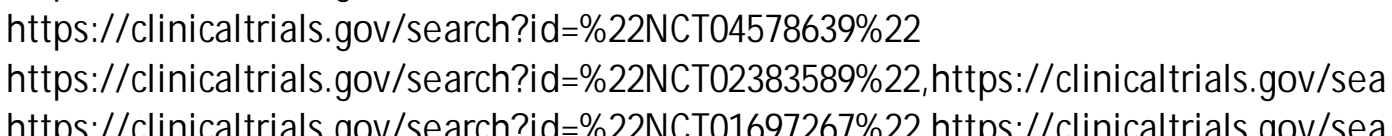

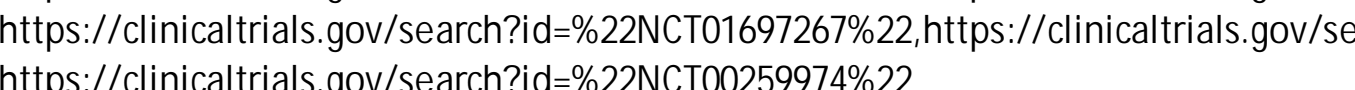

https:///clinicaltrials.gov/search?id=\%22NCT01164098\%22

https://clinicaltrials.gov/search?id=\%22NCT00801281\%22

https://linicaltrials.gov/search?id=2/222NCT00169156\%22

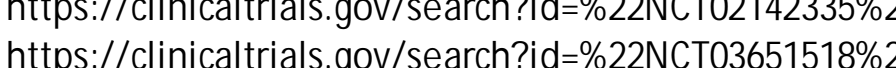

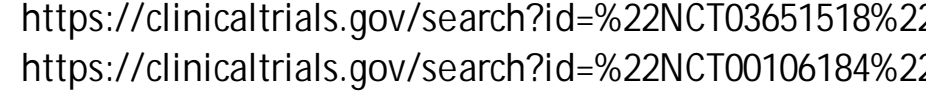

https://clinicaltrials.gov/search?id=\%22NCT00379431\%22

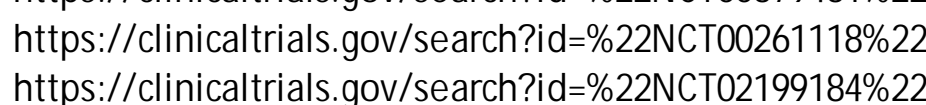


Reduced ejection fraction

2 Not yet recrulting

https://clinicaltrials.gov/search?id_=222NCT03332888\%22

hemolytic-uremic syndrom

$\begin{array}{ll} & \\ & \end{array}$

MONDO_0009348
MONDO-0018170

2 Unknown stat

MONDD_0019139
MONDD_0020743

classic Hodgkin lymphoma

MONDO-0021117

acquired hemophilia

Irphanet__38510 Lymphoproliferative syndrome

$\begin{array}{ll}\text { Orphanet } 797 & \text { Sarcoidosis } \\ \text { Orphanet } 98878 & \text { Hemophilia A }\end{array}$

$\begin{array}{ll}\text { Orphanet_-08878 HemophiliaA } \\ \text { EFO__0000096 } & \text { neoplasm of mature B-cells }\end{array}$

FFO 00001

(FO-0000019

Hodgkins lymphoma
MALT Iymphoma

EF__00000209

EFO_0000220

aute lymphobhobsiastic leukemia

EFO-00000304

te myeloid leukemia

2 Recruiting

https:// clinicaltrials.gov/ search?id=\%22NCT00531089\%22

https://clinicaltrials.gov/ search?id=\%22NCT02533926\% 22

Attps.//clinicaltrials.gov/searchidef22NCT0065732\%22

https:///linicaltrials.gov//search?id=\%222N CT01268033\%22

https:// clinicaltrials.gov/search?id=\%22NCT03700229\%22

2 Unknown status

https:// clinicaltrials.gov/search?id=2\%22NCT02135874\%22

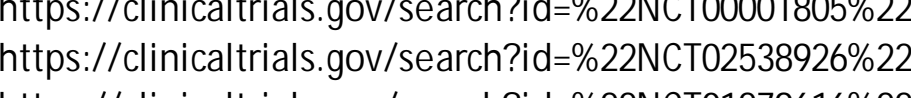

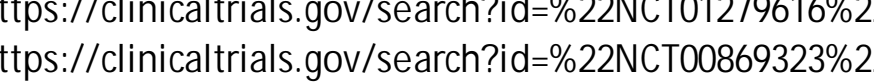

https:// clinicaltrials.gov/search?id=\%22N CT00855205\%22

https:// clinicaltrials.gov/search?id=\%22NCTO0331000\% 22

https // clinicaltrials gov/searchidd-0222NCT02408012\%22

https:///linicaltrials.gov/search?id $=2222 \mathrm{NCT}$ CT00122298\% 222

https://clinicaltrials.gov/search?id=\%22N CT02960646\%22, https://clinicaltrials.gov/sea

ttps:// clinicaltrials.gov/search?id= $=222 \mathrm{NCT} 03808610 \% 22$

Buskitts Iymphoma

cancer
chronic myelogenous leukemia

https:///clinicaltrials.gov/search?id=\%22NCT01371630\%22,
https://clinicaltrials.gov/search?id=\%22NCT02779283\%22

https:///linicaltrials.gov/search?id=\%222NCT03432741\%22

EFO-0000339

(FO-000038

$\mathrm{EFO}-0000565$
$\mathrm{EFO}-0000610$

Crohn's diseaser

leukemia
neoplasm

rheumatoid arthritis

schizophrenia
Sjogren syndrome

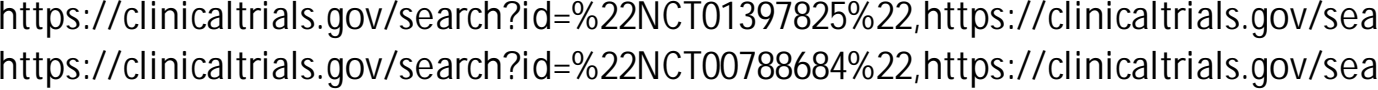

https://lilinicaltrials.gov/search?id=/222N CT016197761\%22

https//dinicaltrials gov/searciid-6222NCT02356150\%22

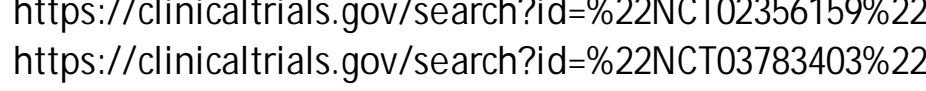

https://clinicaltrials.gov/search?id=\%22NCT03061838\%22,https://clinicaltrials.gov/sea

https:// clinicaltrials.gov/ search?id=\%22N CT03983018\%22 
https://clinicaltrials.gov/search?id=\%22N CT00036855\%22

https:///linicaltrials.gov/search?id= $\% 22 \mathrm{NCT} 00244361 \% 22$

EFO-1001486 primary biliary cirrhosis
EFO-1001779 chronic myelomonocytic leukemia EFO 1001779 kidney failure Abnormality of blood and blood-form Aplastic anemia

lymphoblastic lymphom

$\begin{array}{ll}\text { MONDD_0000023 } & \text { prolymphocytic leukemia } \\ \text { MOND_-_001437 } & \text { puly alveolar proteinosis }\end{array}$

scleritis

hematopoietic and lymphoid system n

(1)

MONDD-0013730 graft versus host disease

DO_0015564

T-cell non-Hodgkin lymphoma

thrombotic thrombocytopenic purpura

MONDO-0019457 therapy related acute myeloid leukem

static malignant neoplasm

MONDO_0044881 hematopoietic and lymphoid cell neor

central nervous system non-hodgkin

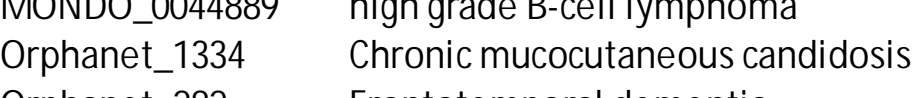

Orphanet_282

Orphanet_-309144 Gangliosidosis

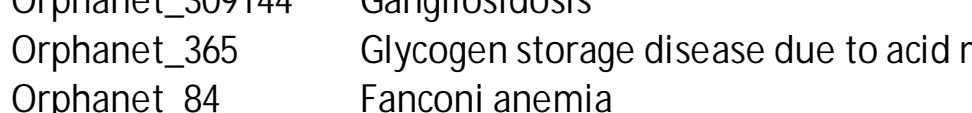

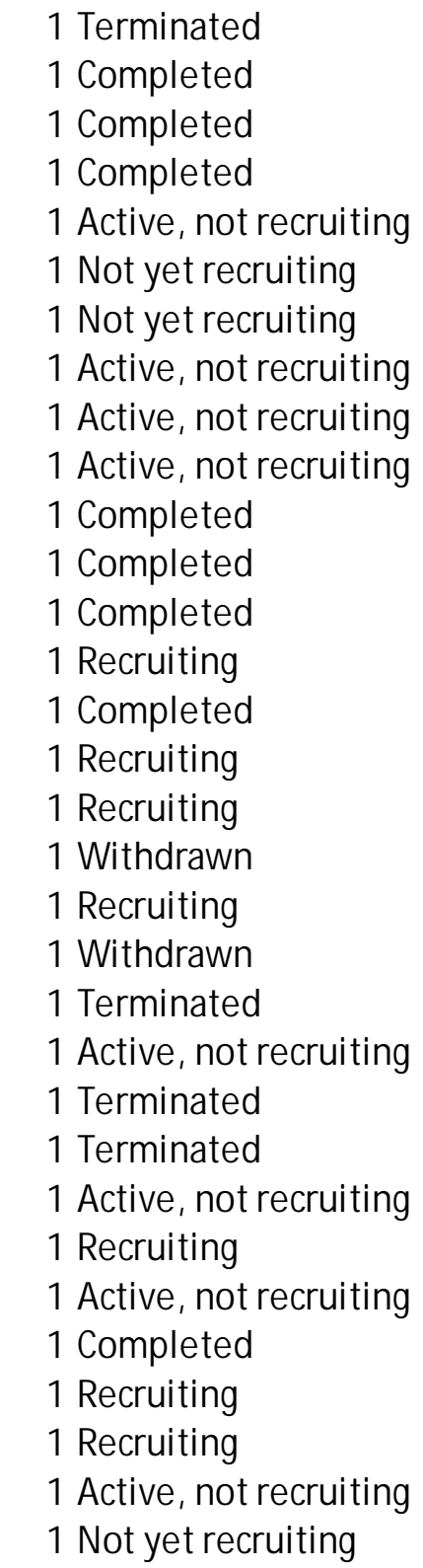

https: // clinicaltrials.gov/search?id $=\% 22 \mathrm{~N} C$ CT00150111\% 22
https://clinicaltrials.gov/ search?id=\%22NCT00364819\%2

,https://clinicaltrials.gov/sea

tttps://clinicaltrials.gov/search?id=\%22NCT02960646\% 22

https://clinicaltrials.gov/search?id= $\% 222 N$ CTO4540380\%22
https://clinicaltrials. gov/search?id=\%22NCT04243434\%22

https://clinicaltrials.gov/search?id= $=222 \mathrm{NCTO292906446 \% 22}$

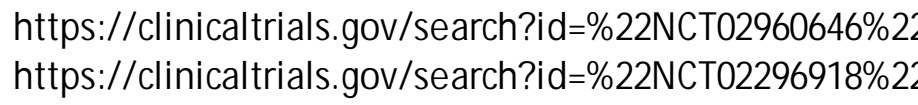

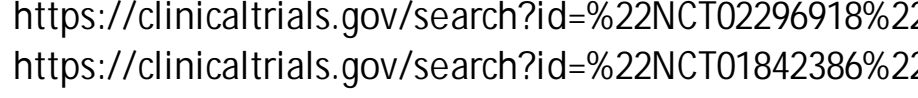

https:/// clinicaltrials.gov/search?id =\%22NCT Co0415506\%22

https:/// clinicaltrials.gov/ search?id=\%22NCT04022239\%22
https://clinicaltrials.gov/search?id=\%22NCT0275613\%22

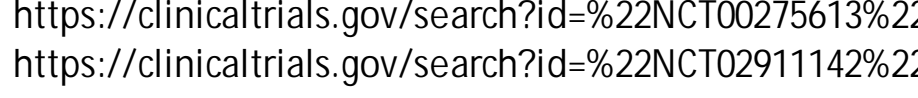

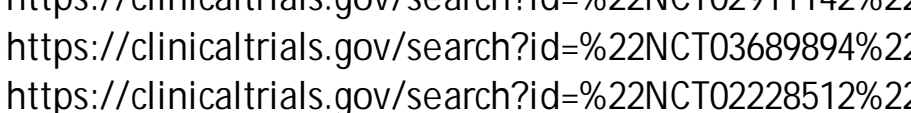

https:// clinicaltrials.gov/ search?id=\%22NCT01701986\% 222

https:///linicaltrials. gov/search?id=\%22NCT00251277\%22

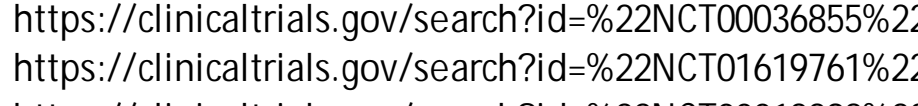

https:// clinicaltrials.gov/search?id= $=022 \mathrm{~N}$ C CT015069127\% 22

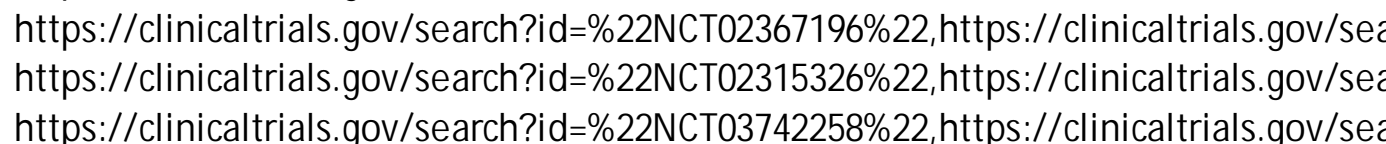

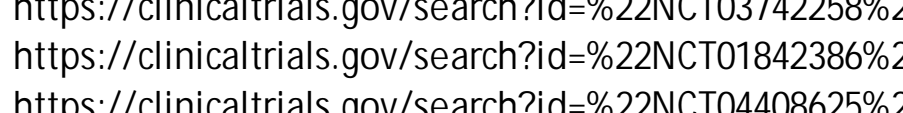

https:// clinicaltrials.gov/search?id=\%22 N CT03952637\% 22

https:///clinicaltrials.sov/ search?id=\%22NCT022240407\%22
https://clinicaltrials.gov/search?id=\%22NCT04784052\%22 


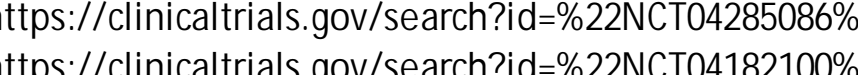

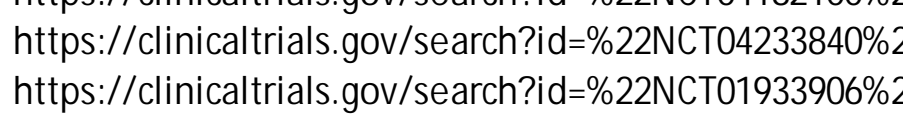

EF__oo000616 neoplasm

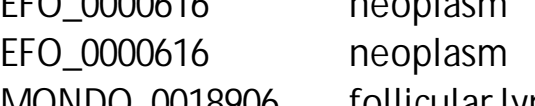

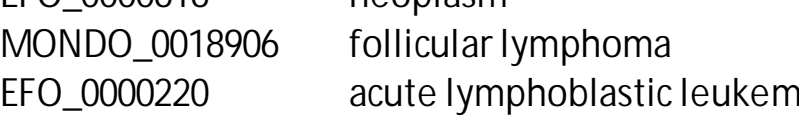

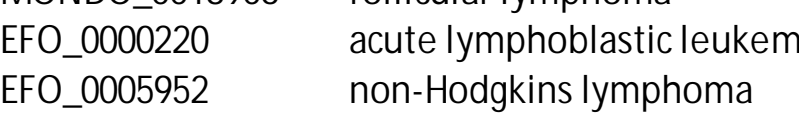

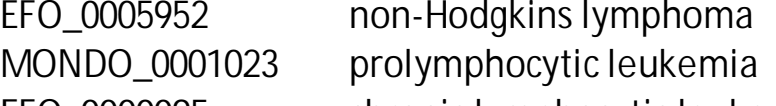


\begin{tabular}{ll} 
CHEMBLL298047 & TAFASITAMAB \\
CHEMBL4298047 & TAFASITAMAB \\
\hline
\end{tabular}

CHEMBL3301574 TISAGENLECLEUCE.

CHEMBL 3301574

CHEMBL3301574 - TISAGENIECLEUC

CHEMBL3301574 TISAGENLECLEUCE

$\begin{array}{ll}\text { CHEMBL3301574 } & \text { TISAGENLECLEUCEL } \\ \text { CHEMBL3301574 } & \text { TISAGENLECLEUCEL }\end{array}$

CHEMB BL3301574 TISAGENLECLEUCE

CHEMBL3301574 TISAGENLECLEUCA

$\begin{array}{ll}\text { CHEMBL3301574 } & \text { TISAGENLECLEUCEL } \\ \text { CHEMBL3301574 } & \text { TISAGENLECLEUCEL }\end{array}$

CHEM BL23301574

CHEM BL3301574 TISAGENLECLEUCE
TISAGENLECEUCE

CHEMBL3301574 TISAGENLECLEUCE

CHEM BL3301574

CHEMBL1201604

CHEMBL1201604 TOSTUMOMAB

TOSTUMOMAB

CHEMB13833304 TOSTMOMAB

CHEMB B38333304 TOSITUMOMAB 13/

CHEM BL3833304 TOSITUMOMAB 13I

CHEMBEB3833304 TOSITMMOMAB B B1
CHEMBB333304

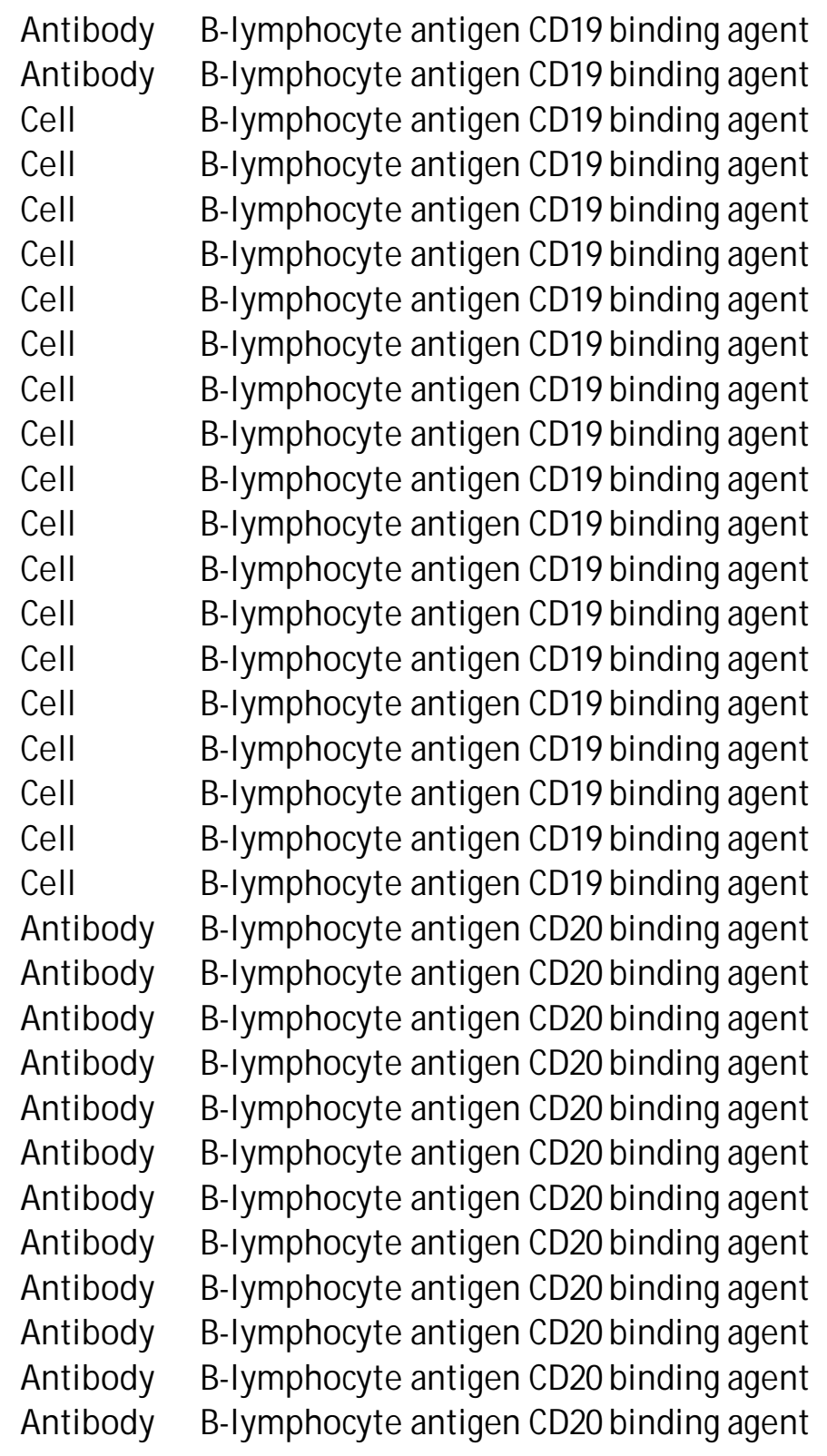

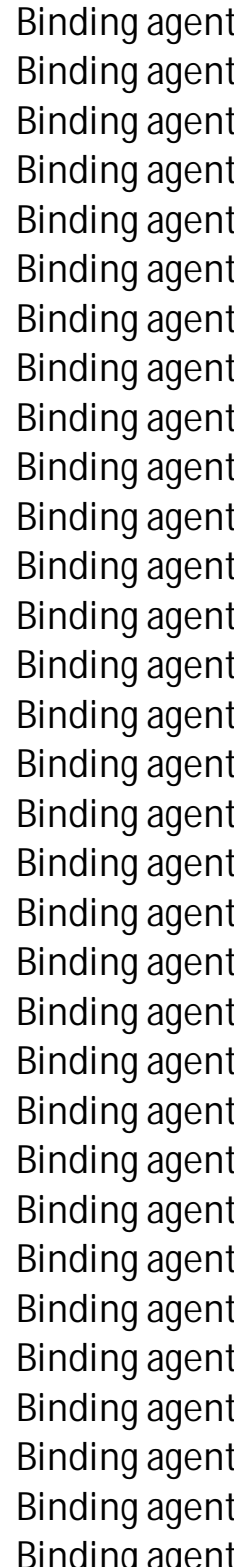

EFO_0000403 diffuse large B-cell lymphoma

lymphoma
neoplasm

1 Active, not recruiting
Not yet recruiting

https:///linicaltrials.gov/search?id=\%22N CT04134936\%22

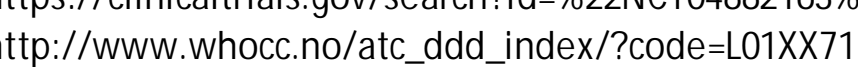

EFO-0000096 neoplasm of mature B-cells

EFO-000428

EFO- 10001469

$\begin{array}{ll}\text { EFO_1001469 } & \text { Mantle cell lymphoma } \\ \text { MONDO__000873 } & \text { Iymphoblastic lymphoma }\end{array}$

EFO 0000095

B-cell acute lymphoblastic leukemia

acronic lymphocytic leukemia

EFO_000056

EFO-0001378

EFO_1000157

EFO-0000574

EFO_0002618

EFO_0000095
EFO-0000574

EFO_1001469

EFO_0000096

MONDO_0018906

EFO- 0000574

EFO-00013032

$\mathrm{EFO}-0000095$
$\mathrm{EFO}-0000096$

leukem

multiple myeloma

(y)

diffuse large B-cell lymphom

lymphoma

pancreatic carcinoma
chronic lymphocytic leukemia

ymphoma

neoplasm of mature B-cells

non-Hodgkins Iymphoma

follicular lymphom
lymphoma

ymphoma
muttiple myelon

EFFo-0000096

naplasticlarge cell lymphoma

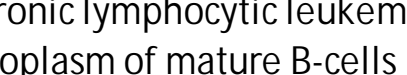

neoplasm of mature $B$-cells
Hodgkins lymphoma
2 Unknown status

2 Active, not recruiting

2 Unknown status

2 Active, not recruiting

1 Unknown status
1 Recruiting

1 Recruiting

1 Terminated

1 Recruiting

Recruiting

0 Terminated
Not yet recruiting

0 Completed

0 Unknown status

2 Completed

1 Completed

1 Completed

2 Completed

2 Active, not recru

1 Unknown status

1 Completed
1 completed https:// clinicaltrials.gov/search?id=\%22NCT03391726\%22

https:// clinicaltrials. gov/search?id=\%22NCT03610724\%222

als.gov/search?id=\%22NCT03321123\%22

https:// clinicaltrials.gov/search?id=\%22NCT03568461\%22

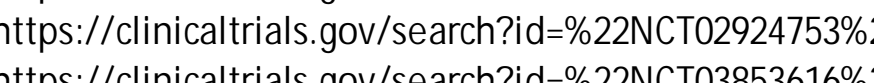

Attps:// clinicaltrials.gov/search?id=\%22NCT01551043\%22

https:// clinicaltrials.gov/search?id=\%22NCT03896854\% 2

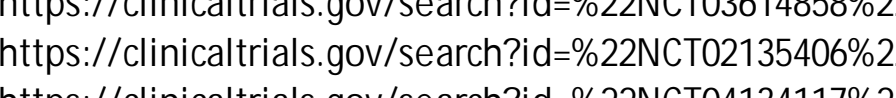

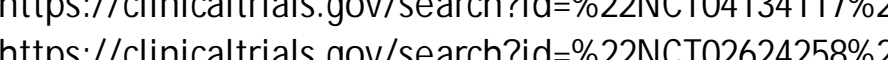

https:///linicaltrials.gov/search?id=\%22NCTO4890236\%22

https:// cinicaltrials.gov/ search?id= $=222 \mathrm{NCTO2476734 \% 22}$

https:///clinicaltrials.gov/search?id=\%22NCT03497819\%22

https:// clinicaltrials.gov/search?id $=222$ N CT00107380\%22

https:///linicaltrials.sov/search?id=\%222NCT00992992\% 22

https:// clinicaltrials.gov/ search?id=\%22NCT00434629\%22

https:// clinicaltrials.gov/search?id=\%22N CT00315731\% 22

https:///linicaltrials.gov/search?id= =222N CT000135200\%22

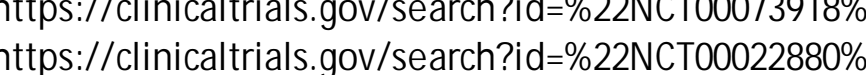

https:// clinicaltrials.gov/ search?id=\%22NCT00434629\%222
https://clinicaltrials.gov/search?id=\%22NCT0048474\%22 
$\begin{array}{ll}\text { CHEM BL3833304 } & \text { TOSITUMOMAB } 1311 \\ \text { CHEMBL3833304 } & \text { TOSITUMOMAB } 1311\end{array}$

CHEMBB3383304 TOSITUMOMAB 131I
CHEMB

CHEMBL3833304 TOSITUMOMAB 1311

CHEMBLL3833304 TOSITUMOMAB 1311

$\begin{array}{ll}\text { CHEMBL23833304 } & \text { TOSITUMOMAB 1311 } \\ \text { CHEMBL3833304 } & \text { TOSITUMOMAB 1311 }\end{array}$

CHEMBL1743083 TREGAUZUMAB

CHEMBL1743083 TREGAUZUMAB

CHEMBLL297845

CHEM BL4297845

CHEM BL4297845
CHEM BL2108354

CHEMBL2108354

CHEMBL2108354

CHEM BL2108354
CHEMBL2108354

CHEM BL2108354

CHEMBL210835

CHEMBL3137347

CHEMBLL1373347
CHEMBL3137347

CHEMBL3137347

CHEMBL3137347

CHEMBL31373347

TRU-015

RUU-015

UBLTUXIMAB
UBUTUXIMAB
UBUTUXIMAB

UBUTUXIMAB

UBUTUXIMAB

UBLTUXIMAB
UBUTUXIMAB
UBUTUXIMAB

UBUTUXIMAB

VARLLUMAB

VARLLUMAB

VARLLUMAB

VARLLUMAB

VARLLUMAB
VARLLUMAB

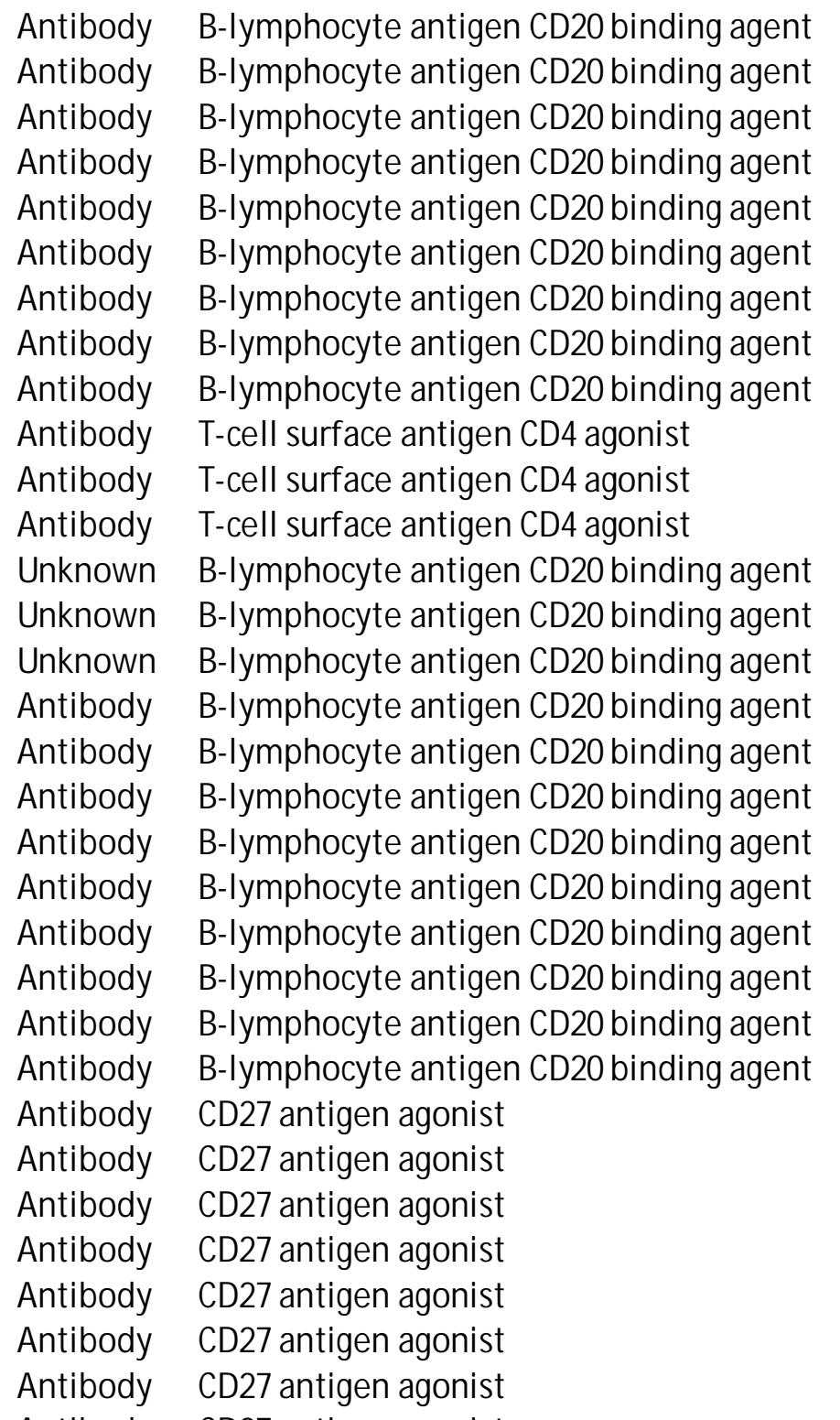

$\begin{array}{ll}\text { Antibody } & \text { CD27 7antigen agonist } \\ \text { Antibody } & \text { CD27 antigen agonist }\end{array}$

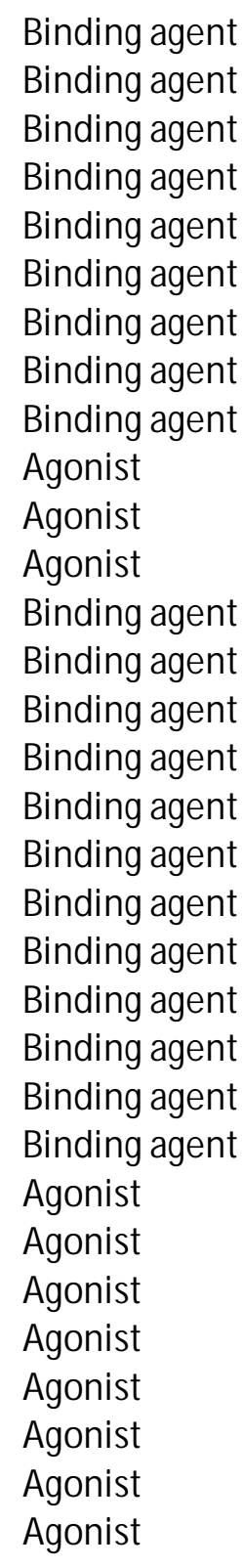

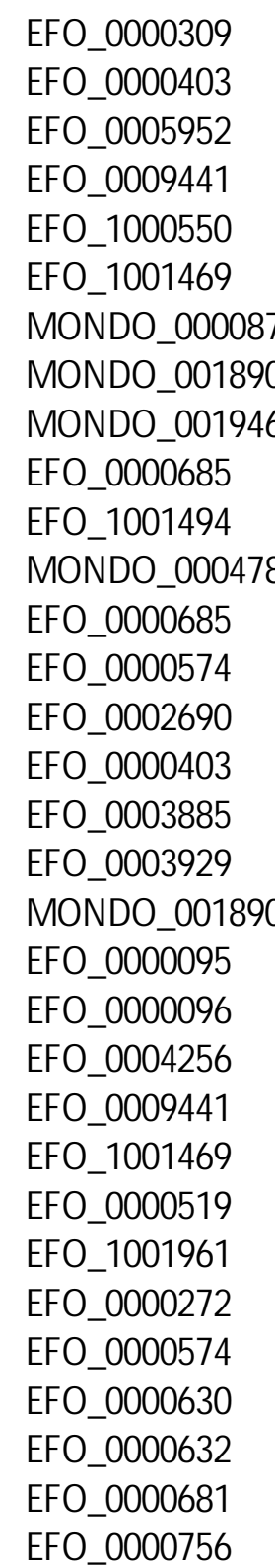

\begin{tabular}{|c|}
\hline 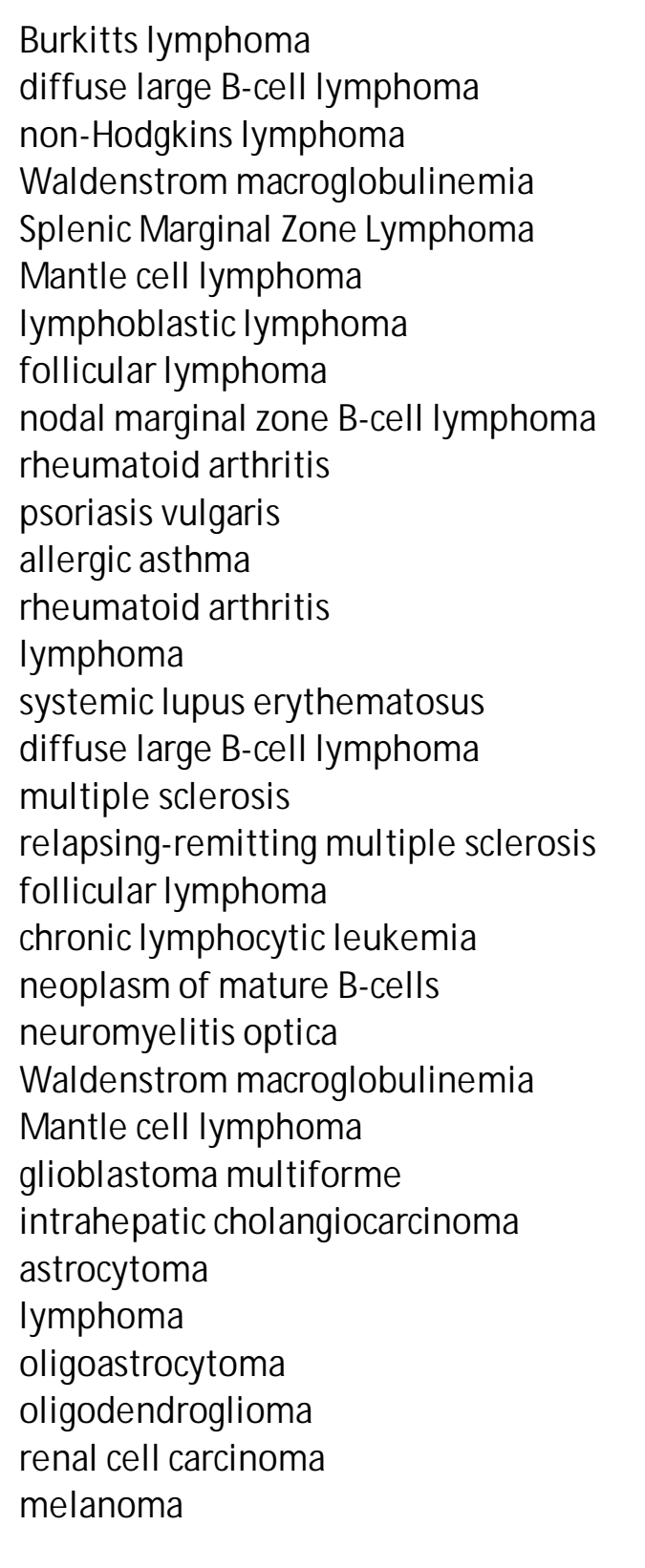 \\
\hline
\end{tabular}

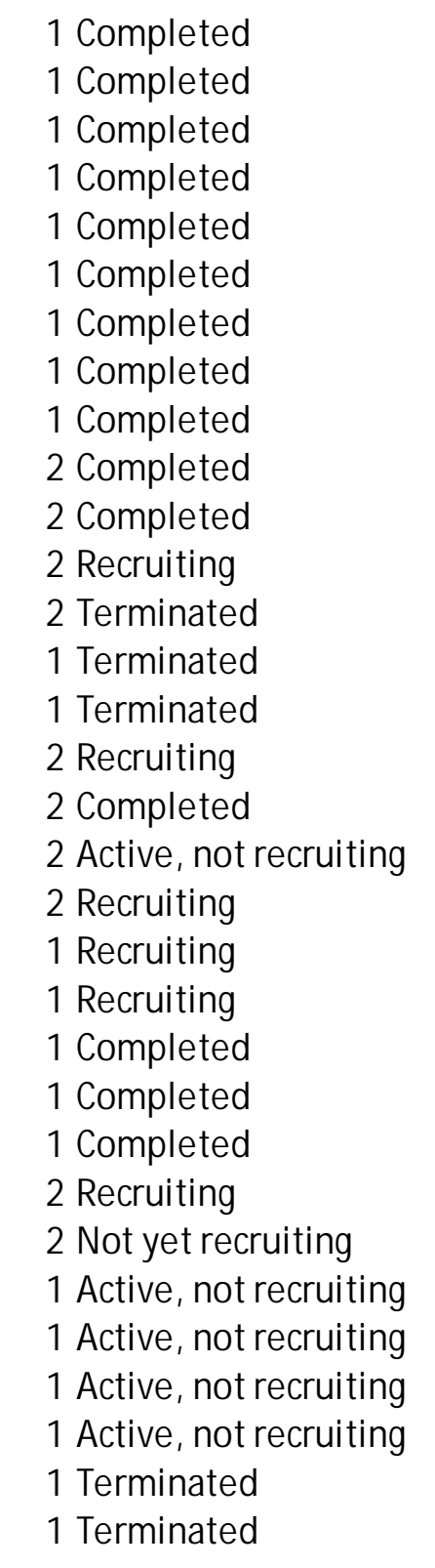

https:///linicaltrials.gov/search?id=/222NCT00110071\%22
https:// clinicaltrials.gov/search?id=/222NCT00110071\%22

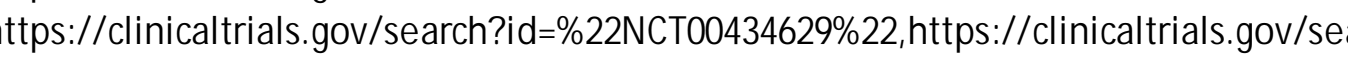
https:// clinicaltrials.gov/ search?id $=\% 22 \mathrm{NCTO0110071 \% 22}$ https://dinicaltrials gov/search?id-2022NCT00110071\%22 https:///linicaltrials.gov/search?id=\%22N CTO0110071\%22

https://clinicaltrials.gov/search?id=\%22N Cro0110071\%22, https://clinicaltrials.gov/sea https:// clinicaltrials.gov/search?id=\%22NCT00110071\%22 https:// clinicaltrials.gov/search?id=222NCT01481493\%22 https://clinicaltrials gov/searchiddef22N NCT04673591\%22 https://clinicaltrials.gov/search?id=1222NCTO0634933\%22 https://clinicaltrials.gov/search?id=\%22NCT00052163\%2 22
https:///linicaltrials.gov/search?id=\%22NCT00479622\%22

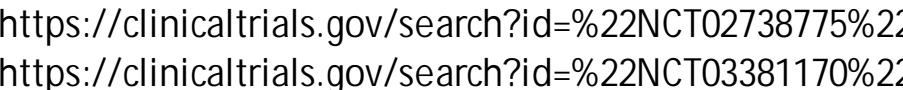
https:// cinicaltrials.gov/search?id=\%22NCT03828448\%22, https:// clinicaltrials.gov/sea https://clinicaltrials.gov/search?id=\%222NCT03671590\%22, https:///clinicaltrials.gov/sea

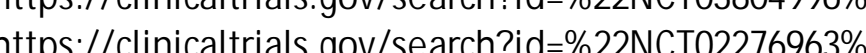
https://clinicaltrials gov/search2id-\%22 N CT01744912\%22 https://clinicaltrials.gov/search?id=\%22N CT01744912\% 22 https:// clinicaltrials.gov/search?id=\%22NCT03688178\%22 https://clinicaltrials.gov/search?id=e222NCT04941287\%22 https://dinicaltrials gov/searchident22NCT03307746\%22 https:///linicaltrials.gov/search??d=\%222NCC103307746\%22 https:// clinicaltrials.gov/search?idd $=222 \mathrm{NCT} 02924038 \% 22$ https:///linicaltrials.gov/search?id=\%22NCT02386111\%22
https://clinicaltrials.gov/search?id=\%22NCT02413827\%22 
lymphoma 



\section{FinnGen}

Aarno Palotie

Mark Daly

Bridget Riley-Gills

Dirk Paul

Athena Matakidou

Adam Platt

Heiko Runz

Sally John

George Okato

George Okafo

Robert Plenge

Robert Plenge

Joseph Marann

Mark McCarthy

Julie Hunkapiller

Margaret G. Eh

Kirsi Auro

Simonne Longerich

Caroline Fox

Katherine Klinger

Deepak Raipal

Eric Green

Robert Graham

Robert Yang

Chris O'Donnell
Tomi P. Mäkelä

Jaakko Kaprio

Antti Hakanen
Affiliation

institute for Molecular Medicine Finland (FIMM), HiLlFE, University of Helsinki, Helsink , Institute for Molecular Medicine Finland (FIMM), HiLIFE, University of Helsinki, Helsinki, Abbvie, Chicago, IL, United States

Abvie, Chicago, IL, United States

Astra Zeneca, Cambridge, United Kingdom

Astra Zeneca, Cambridge, United Kingdom

Astra Zeneca, Cambridge, United Kingdom

Biogen, Cambridge, MA, United States

Biogen, Cambridge, MA, United States

Boehringer Ingelheim, Ingelheim am Rhein, Germany

Bohring

Bristol Myers Squib, New York, NY, United States

Ber

Ger

Genentech, San Francisco, CA, United States

GlaxoSmithkline, Collegeville, PA, United States

GlaxoSmithKline, Espoo, Finland

Merck, Kenilworth, NJ, United States

Pfizer, New York, NY, United States

Translational Sciences, Sanofi R\&D, Framingham, MA, USA
Translational Sciences, Sanofi R\&D, Framingham, MA, USA

Maze Therapeutics, San Francisco, CA, United States

Maze Therapeutics, San Francisco, CA, United States

Janssen Biotech, Beerse, Belgium

Hovartis Institutes for BioMedical Research,

Cambridge, MA, United States

IFE, University of Helsinki, Helsinki, Finlan

Auria Biobank / University of Turku / Hospital District of Southwest Finland, Turku, Finland
Auria Biobank / University of Turku / Hospital District of Southwest Finland, Turku, Finland bridget.rileygillis@abbvie.com

howard.jacob@abbvie.com

dirk.paul@astrazeneca.com

athena.x.matakidou@gsk.com

adam.platt@astrazeneca.com

heiko.runz@biogen.com

sally.john@biogen .com

george.okafo@boehringer-ingelheim.com

nathan.lawless@boehringer-ingelheim.com

robert.plenge@bms.com

joseph.maranville@bms.com

mccarthy.mark@gene.com

hunkapiller.julie@gene.com

meg.g.ehm@gsk.com

si.m.auro@gsk.com

simonne.longerich@merck.con

caroline.tox@merck.com

katherine.klinger@sanoficom

deepak.rajpal@sanofi.com

egreen@mazetx.com

graham@mazetx.com

yang31@its.jnj.com

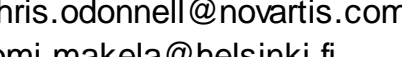

kaprio@hesinkif

petri. virolainen@tyks.
Role 1

teering Committee

teering Committee

Steering Committee

Steering Committee

Steering Committee

Steering Committee

Steering Committee

Steering Committee

ing Committee

Steering Committee

cornte

Steering Committee

seering Committee

teering Committee

Steering Committe

Steering Committe

Steering Committe

Steering Committee

Steering Committe

Steering Committee

Steering Committee

Steeng Commitre

Steering Committee
Role 2

Steering Committee

Steering Committee

rmaceutical companies

Pharmaceutical companies

Pharmaceutical companies

Pharmaceutical companie

Pharmaceutical companies

Pharmaceutical companies

Pharmaceutical companies

Pharmaceutical companies

(compantes

Pharmaceutical companies

Pharmaceutical companies

Pharmaculical companies

Pharmace

Pharmaceutical companies

Pharmaceutical companies

University of Helsink \& Bobanks

University of Helsinki \& Biobanks

(1)


Terhi Kilpi Markus Perola
Jukka Partanen Jukka Partanen Anne Pitkäranta Juhani Junttila

Raisa Serpi

Tarja Laitinen

Veli-Matti Kosma Jari Laukkanen

Marco Hautalaht Raimo Pakkane Jeffrey Waring Bridget Riley-Gilli
Fedik Rahimov Fedik Rahimov Chi-Yen Chen Chia-Yen Chen Zhihao Ding Marc Jung

Shameek Biswas Rion Pendergrass Julie Hunkapiller Margaret G. E Neha Raghan Adriana Huertas-Vazquez Jae-Hoon Sul
Anders Mälarstig

Anders Malarstig
THL Blobank / Finnish Institute for Health and Welfare (THL), Helsinki, Finland

terhi.kilpi@thl.fi Finnish Red Cross Blood Service / Finnish Hematology Registry and Clinical Biobank, Helsinki, jukka.partanen@veripalvelu. Helsinki Biobank/ 列 Northern Finland Biobank Borealis / University of Oulu / Northern Ostrobothnia Hospital District, raisa.serpi@ppshp.fi Oulu, Finland Finnish Clinical Tampere, Finland Biobank of Easten

Finland / University of Eastern Finland / Northern Savo Hospital Distric

tarja.laitinen@pshp.fi

veli-matti.kosma@uef.fi

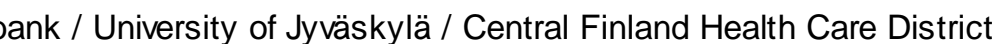

jari.laukkanen@ksshp.fi

Jyväskylä, Finland

Fisiness Finland, Helsinki, Finland

Business Finland, Helsinki, Finland

Abbvie, Chicago, IL, United States

Abbve, Chicago, IL, United States

Abbvie, Chicago, L, United States

Bstra Zeneca, Cambridge, United Kingdom

Biogen, Cambridge, MA, United States

Boehringer Ingelheim, Ingelheim am Rhein, Germany

Boehringer Ingelheim, Ingelheim am Rhein, Germany

Bristol Myers Squibb, New York, NY, United States

Genentech, San Francisco, CA, United States

(a)

(a)

Merk, Kenilworth NW, United States

Merck, Kenilworth, NJJ, United States marco.hautalahti@finbb.fi

outi.tuovila@businessfinland.fi

raimo.pakkanen@businessfinland.f

jeff.waring@abbvie.com

bridget.rileygillis@abbvie.com

redik.rahimov@abbvie.com

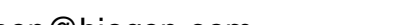

heiko.runz@biogen.com

zhihao.ding@boehringer-ingelheim.com

marc_oliver.jung@boehringer-ingelheim.com

Shameek.Biswas@bms.com

penders2@gene.com

hunkapiller.julie@gene.co
meg.g.ehm@gsk.com

meg.g.ehm@gsk.com

neha.raghavan@merck.com
adriana.huertas.vazquez@merck.com

jae.hoon.sul@merck.com

anders.malarstig@pfizer.com
Steering Committee

Steering Committe

Steering Committe

Steering Committe

Steering Committee

Steering Committee

Steering Committee

Steering Committee

Steering Committee

Steering Committe

Steering Committee

Scientific Committe

Scientific Committee

Scientific Committee

Scientific Committe

scientific committee

Scientific Committe

Scientific Committe

Scientific Committe

Scientific Committe

Scientific Committee

Scientific Committee

cientific Committee

Scientific Committe

Scientific Committere

Scientific Committee
University of Helsinki \& Biobanks University of Helsink \& B Blobanks University of Helsinki \& Biobanks

University of Helsinki \& Biobanks University of Helsinki \& Biobanks

University of Helsinki \& Biobanks

University of Helsinki \& Biobanks University of Helsinki \& Biobanks University of Helsinki \& Biobanks

University of Helsinki \& Biobanks Other Experts/ Non-Voting Members
Other Experts/ Non-Voting Members

Pharmaceutical companies

Pharmaceutical companies

Pharmaceutical companies

Pharmaceutical companies

Pharmaceutical companies

Pharmaceutical companie

Pharmaceutical companies

Pharmaceutical companies

Pharmaceutical companies

Pharmaceutical companies

Pharmaceutical companies

Pharmaceutical companie

Pharmaceutical companies

Pharmaceutical companies

Pharmaceutical companies 
Nicole Renaud

Ma en Obeidat

Johanna Schleutker

Markus Perola
Mikko Arvas

Olli Carpén

Johannes Kettunen

Arto Mannermaa

Katriina Aalto-Setälä

Jari Laukkanen

Johanna Mäkelä

Reetta Kälväinen

Valtteri Julkunen

Hilkka Soininen

Anne Remes
Mikko Hiltunen

Jukka Peltola

Jukka Peltola
Minna Raivio

Pentti Tienari

Juha Rinne
Pfizer, New York, NY, United States

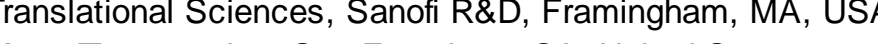

Maze Therapeutics, San Francisco, CA, United States

Maze Thapelics, San Frncisco, CA, Unied Slates

Jane

Novartis Institutes for BioMedical Research Cambridge, PA, United States

Institute for Molecular Medicine Finland (FIMM), HiLIFE, University of Helsinki, Helsinki, Finland

Auria Biobank / Univ. of Turku / Hospital District of Southwest Finland, Turku, Finland

THL Biobank / Finnish Institute for Health and Welfare (THL), Helsinki, Finland
Finnish Red Cross Blood Service / Finnish Hematology Registry and Clinical Biobank, Helsinki,

Finland

Helsinki Biobank / Helsinki University and Hospital District of Helsinki and Uusimaa, Helsinki

Northern Finland

Northern Finland Biobank Borealis / University of Oul / Northern Ostrobothnia Hospital District, johannes, kettunen@oul

Oulu, Finland Biobank of Eastern Finland / University of Eastern Finland / Northern Savo Hospital District,

Faculty of Medicine and Health Technology, Tampere University, Tampere, Finland

Finnish Clinical Biobank Tampere / University of Tampere / Pirkanmaa Hospital Distric

Tampere, Finland

Jyväskylä, Finland

FINBB - Finnish biobank cooperative

Northern Savo Hospital District, Kuopio, Finland

Northern Savo Hospital District, Kuopio, Finland

Northern Savo Hospltal Distric, Kuopio, Finland

University of Eastern Finand Kuopio, Finlu, Fin

Pirkanmaa Hospital District, Tampere, Finland

Hospital District of Helsinki and Uusimaa, Helsinki, Finland

Hospital District of Helsinki and Uusimaa, Helsinki, Finland

Hospital District of Southwest Finland, Turku, Finland muli.ripatti@helsinki.fi

johanna.schleutker@u

ikko.arvas@veripalvelu.

li.carpen@helsinki.f

arto.mannermaa@uef.fi

katriina.aalto-setala@tuni.fi

a.kahonen@uta.fi

jari.laukkanen@ksshp.fi

johanna.makela@inbb.fi

reetta.kaluainen@kuh.fi

valtteri.julkunen@kuh.fi

hilkka.soininen@uet.f

anne.remes@oulu.f

jukka.peltola@pshp.

minna.raivi@geri.fi

pentti.tienari@hus.fi

juha.rinne@tyks.fi
Scientific Committee

Scientic Committe

Scientific Committee

Scientific Committee

Scientific Committee

Scientific Committee

Scientific Committee

Scientific Committee

scientific Committee

Scientific Committee

Scientific Committee
Scientific Committee

Scientific Committee

Scientific Committee

Scientific Committee

Scientific Committee

Scientific Committee

Clinical Groups

Clinical Groups

Clinical Groups

Clinical Groups

Clinical Groups

Clinical Groups

Clinical Groups

Clinical Groups
Pharmaceutical companies

Pharmaceutical companies

Pharmaceutical companies

Pharmaceutical companies

Pharmaceutical companies

Pharmaceutical companies

Pharmaceutical companies

University of Helsinki \& Biobanks

University of Helsinki \& Biobanks

University of Helsinki \& Biobanks

University of Helsinki \& Biobanks

University of Helsinki \& Biobanks

University of Helsinki \& Biobanks

University of Helsinki \& Biobanks

University of Helsinki \& Biobanks

University of Helsinki \& Biobanks University of Helsinki \& Biobanks

University of Helsinki \& Biobanks

University of Helsinki \& Biobanks

Neurology Group

Neurology Group

Neurology Group

Neurology Group

Neurology Group

Neurology Group

Neurology Group

Neurology Group 
Roosa Kallionpää
Juulia Partanen

Juulia Partanen
Ali Abbasi

Adam Ziemann

Nizar Smaoui

Susan Eaton

Heiko Runz

Sanni Lahdenperà

Shameek Biswas

Julie Hunkapills

Natalie Bowers

Edmond Teng
Rion Pendergrass

Fanli $X_{u}$

Fanli Xu
David Pulford

Kirsi Auro

Laura Addis

John Eicher

Qingain S L

Kkater He

Neha Raghavan

Martti Färkkilä
Jukka Koskela

Sampsa Pikkarainen

Airi Jussila

Katri Kaukinen

Timo Blomster

Markku Voutiliainen

Markk Daly

Ali Abbasi
Jeffrey Warin
Hospital District of Southwest Finland, Turku, Finland

Astitute for Molecular Medicine Finland, HiLlFE, University of Helsinki, Finland

Abbvie, Chicago, IL, United States

Abvie, Chicago, II, United States

Abbri, Chicago It. United States

Biogen, Cambridge, MA, United States

Biogen, Cambridge, MA, United States

Biogen, Cambridge, MA, United States
Bristol Myers Squibb, New York, NY, United States

Genentech, San Francisco, CA, United States

Genentech, San Francisco, CA, United States

Genentech, San Francisco, CA, United States

GlaxoSmithKline, Brentford, United Kingdom

GlaxoSmithKline, Stevenage, United Kingdom

GlaxoSmithKline, Espoo, Finland

GlaxoSmithKline, Brentiord, United Kingdom

Janssen Research \& Development, LLC, Titusville, NJ 08560, United States

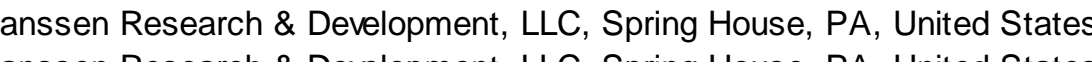

LC, Spring House, PA, United States

Kenilworth, NJ, United States

Hospital District of Helsinki and Uusimaa, Helsinki, Finland

Hospital District of Helsinki and Uusimaa, Helsinki, Finland
Hospital District of Helsinki and Uusimaa, Helsinki, Finland

Pirkanmaa Hospital District, Tampere, Finland

Pirkanmaa Hospital District, Tampere, Finland

Northern Ostrobothnia Hospital District, Oulu, Finlan

Hospital Sistrict of Southwest Finland, Tunk Find

Institute for Molecular Medicine, Finland (FIMM), HiLlFE, University of Helsinki, Helsink

Institute for Molecular Medicine, Finland (FIMM), HiLIFE, University of Helsin
Finland; Broad Institute of MIT and Harvard; Massachusetts General Hospital

Abbvie, Chicago, IL, United States
Abbvie, Chicago, IL, United States roosa.kallionpaa@tyks.fi

ali.abbasi@abbvie.com

adam.ziemann@abbvie.com

nizar.smaoui@abbvie.com

susan.eaton@biogen.com

heiko.runz@biogen.com

sanni.lahdenpera@biogen.com

shameek.biswas@bms.com

hunkapiller.julie@gene.com

bowersn1@gene.com

teng.edmond@gene.com

chun-fang 2 ru.com

david.x.pulford@gsk.com

kirsi.m.auro@gsk.com

laura.x.addis@gsk.com

john.d.eicher@gsk.com

QLi2@its.jnj.com

ekhramts@its.jni.com

ekhramts@its.jnj.com

martti.farkkila@hus.fi

jukka.koskela@helsinki.fi

sampsa.pikkarainen@hus.fi

airi.jussila@pshp.fi

katri.kaukinen@tuni.fi

timo.blomster@ppshp.ff

markku.voutilainen@tyks.
mark.daly@helsinki.fi

ali.abbasi@abbvie.com

jeff.waring@abbvie.com
Clinical Groups

Clinical Groups

Clinical Groups

Clinical Groups

clinical Groups

Clinical Groups

Clinical Groups

Clinical Groups

Clinical Groups

Clinical Groups

Clinical Groups

Clinical Groups

Clinical Groups

Clinical Groups

Clinical Groups

Clinical Groups

Clinical Groups

Clinical Groups

Clinical Groups

Clinical Groups

Clinical Groups

Clinical Groups

Clinical Groups

Clinical Groups

Clinical Groups

Clinical Groups

clincal Groups

Clinical Groups

Clinical Groups

Clinical Groups
Neurology Group

Neurology Group

Neurology Group

Neurology Group

Neurology Group
Neurology Group

Neurology Group

Neurology Group

Neurology Group

Neurology Group
Neurology Group

Neurology Group

Neurology Group

Neurology Group

Neurology Group
Neurology Group

Neurology Group

Neurology Group

Neurology Group

Neurology Group

Neurology Group

Neurology Group

Neurology Group
Neurology Group

Gastroenterology Group

Gastroenterology Group

Gastroenterology Group

Gastroenterology Group

Gastroenterology Group

Gastroenterology Group

Gastroenterology Group

Gastroenterology Group

Gastroenterology Group

Gastroenterology Group 
Nizar Smaoui
Fedik Rahimov

Anne Lehtonen

Tim Lu

Natalie Bowers
Rion Pendergrass

Rion Pendergrass
Linda McCarthy

Amy Hart

Mejijan Guan
Jason Miller

Kirsi Kalpala
Melissa Miller

Xnli Hu

Kari Eklund

Antti Palomäk
Pia Isomäki

Laura Pirilà

Oili Kaipiainen-Seppänen

Johanna Huhtakangas

Nina Mars

Jeffrey Waring

Fedik Rahimov

Apinya Lertratana
Nizar Smaoui

Anne Lehtonen

David Close

Marla Hochfeld
Natalie Bowers

Rion Pendergrass

Jorge Esparza Gordilo

Kirsi Auro

Dawn Waterworth
Fabiana Farias

Kirsi Kalpala
Abbvie, Chicago, IL, United States

Abbvie, Chicago, LL, United States

Genentech, San Francisco, CA, United States

Genentech, San Francisco, CA, United States

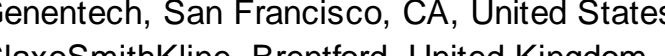

Janssen Research \& Development, LLC, Spring House, PA, United States

Janssen Research \& Development, LLC, Spring House, PA, United States

Merck, Kenilworth, NJ, United States

Pfizer, New York, NY, United States
Pfizer, New York, NY, United States

Pfizer, New York, NY, United States

Hospital District of Helsinki and Uusimaa, Helsinki, Finland

Hospital District of Southwest Finland, Turku, Finland

Pirkanmaa Hospital District, Tampere, Finland

Hospital District of Southwest Finland, Turku, Finland

Northern Savo Hospital District, Kuopio, Finland

Northern Ostrobothnia Hospital District, Oulu, Finland
Institute for Molecular Medicine Finland (FIMM), HiLIFE, University of Helsinki, Helsinki, Finland Abbvie, Chicago, LL, United States

Abve, Chicago, LL, United States

Abbvie, Chicago, IL United States

Abbvie, Chicago, IL, United States

Abbvie, Chicago, IL, United States

Astra Zeneca, Cambridge, United Kingdom

Bristol Myers Squibb, New York, NY, United States

Genentech, San Francisco, CA, United States

GlaxoSmithKline, Brentord United Kingdom

GlaxoSmithKline, Espoo, Finland
Janssen Research \& Development

Kingdom

Plent, LLC, Spring House, PA, United States

Pfizer, New York, NY, United States nizar.smaoui@abbvie.com

fedik.rahimov@abbvie.com

anne.lehtonen@abbvie.com

bowersn1@gene.com

linda.c.mccarthy@gsk.com

ahart13@its.jnj.com

jason.miller4@merck.com

kirsi.kalpal@@pfizer.com

melissa.r.miler@pfizer.com

xinli.hu@pfizer.com
kari.eklund@hus.fi

ajpalı@utu.fi

pia.isomaki@pshp.fi
laura.pirila@fimnet.fi,laura.pirila@tyks.

oili.kaipiainen-seppanen@kuh.fi

johanna.huhtakangas@kuh.fi

ali.abbasi@abbvie.com

jeft.waring@abbvie.com

apinya.lertratanakul@abbvie.com

nizar.smaoui@abbvie.com

anne.lehtonen@abbvie.com

david.close@astrazeneca.com

mhochreld@celgene.com

bowersn1@gene.com

jorge.x.esparza-gordillo@gsk.com

kirsi.m.auro@gsk.com

dwaterwo@its.jni.com

fabiana.farias@merck.com

kirsi.kalpala@pfizer.com
Clinical Groups

Clinical Groups

Clinical Groups

Clinical Groups

clinical Groups

Clinical Groups

Clinical Groups

Clinical Groups

Clinical Groups

Clinical Groups

Clinical Groups

Clinical Groups

Clinical Groups

Clinical Groups

Clinical Groups

Clinical Groups

Clinical Groups

Clinical Groups

Clinical Groups

Clinical Groups

Clinical Groups

Clinical Groups

Clinical Groups

Clinical Groups

Clinical Groups

Clinical Groups
Clinical Groups

Clinical Groups

Clinical Groups

Clinical Groups

Clinical Groups

Clinical Groups
Gastroenterology Group

Gastroenterology Group

Gastroenterology Group

Gastroenterology Group

Gastroenterology Group

Gastroenterology Group

Gastroenterology Group

Gastroenterology Group

Gastroenterology Group

Gastroenterology Group

Gastroenterology Group

Rheumatology Group

Rheumatology Group

Rheumatology Group

Rheumatology Group

Rheumatology Group

Rheumatology Group

Rheumatology Group

Rheumatology Group

Rheumatology Group

Rheumatology Group

Rheumatology Group

Rheumatology Group

Rheumatology Group

Rheumatology Group

Rheumatology Group

Rheumatology Group

Rheumatology Group

Rheumatology Group

Rheumatology Group

Rheumatology Group 
Nan Bing

Tarja Laitinen

Margit Pelkone

Paula Kauppi
Hannu Kankaanranta

Terttu Hariu

Rittta Lahesmaa
Nizar Smaoui

Alex Mackay

Susan Eaton

Hubert Chen

Rion Pendergrass
Natalie Bowers

Joanna Betts

Kirsi Auro

Rajashree Mishra

Majd Mouded

Debby Ngo

Felix Vaura

Veikko Saloma

Kaj Metsärinne

Jenni Aittokallio

Mika Kähönen

Jussi Hernesniem

Daniel Gordin

Murija-Riitta Taskinen

Tinamaija Tuomi

Tinamaija Tuon
Timo Hiltunen

Jari Laukkanen
Pfizer, New York, NY, United States

Pirkanmaa Hospital District, Tampere, Finland

Orthern Savo Hospila Distic, Kuopio, Finlan

and Uusimaa, Helsinki, Finland

作

Northern Ostrobothnia Hospital District, Oulu, Finland

Abbvie, Chicago, IL, United States

Astra Zeneca, Cambridge, United Kingdom

Astra Zeneca, Cambridge, United Kingdo

Glogen, Cambinge, MA, United Slates

(anentech,

Genentech, San Francisco, CA, United States

GlaxoSmithKline, Brentford, United Kingdom

GlaxoSmithKline, Espoo, Finland

GlaxoSmithKline, Brentford, United Kingdom

Novartis, Basel, Switzerland

Finnish Institute for Health and Welfare (THL), Helsinki, Finland

Finnish Institute for Health and Welfare (THL), Helsinini, Finnland

Hospital District of Southwest Finland, Turku, Finland

Hospital District of Southwest Finland, Turku, Finland

Pirkanmaa Hospital District, Tampere, Finland

Pirkanmaa Hospital District, Tampere, Finland
Hospital District of Helsinki and Uusimaa, Helsinki, Finland

Hospita District of Helsinki and Ussima, Hesink, Finand

Hospital District of Helsinki and Uusimaa, Helsinki, Finland

Hospital District of Helsinki and Uusimaa, Helsinki, Finland

Central Finland Health Care District, Jyväskylä, Finland nan.bing@pfizer.com

xin.hepizer.com

margit.pelkonen@kuh.

paula.kauppi@hus.fi

hannu.kankaanranta@tuni.fi

terttu.harju@oulu.fi

rilahes@utu.fi

nizar.smaoui@abbvie.com

alex.mackay@astrazeneca.com

glenda.lass@astrazeneca.co

susan.eaton@biogen.co

penders2@gene.com

bowersn1@gene.com

joanna.c.betts@gsk.com

kirsi.m.auro@gsk.com

rajashree.x.mishra@gsk.com

majd.mouded@novartis.com

debby.ngo@novartis.com

fechva@utu.fi

veikko.salomaa@thl.fi
kaj.metsarinne@tyks.fi

jemato@utu.fi

mika.kahonen@uta.fi

jussi.hernesniemi@tuni.

daniel.gordin@hus.fi

marja-riitta.taskinen@helsinki.fi

tiinamaijatuomi@hus fi

tiinamaij.tuomi@hus.f
timo.hiltunen@hus.fi

jari.laukkanen@ksshp.fi
Clinical Groups

Clinical Groups

Clinical Groups

Clinical Groups

clinical Groups

Clinical Groups

Clinical Groups

Clinical Groups

Clinical Groups

Clinical Groups

Clinical Groups

Clinical Groups

Clinical Groups

Clinical Groups

Clinical Groups

Clinical Groups

Clinical Groups

Clinical Groups

Clinical Groups

Clinical Groups

Clinical Groups

Clinical Groups

Clinical Groups

Clinical Groups

Clinical Groups

clinical Groups

Clinical Groups

Clinical Groups

Clinical Groups
Rheumatology Group

Pulmonology Group

Pulmonology Group

Pulmonology Group

Pulmonology Group

Pulmonology Group

Pulmonology Group

Pulmonology Group

Pulmonology Group

Pulmonology Group

Pulmonology Group

Pulmonology Group

Pulmonology Group

Pulmonology Group

Pulmonology Group

Pulmonology Group

Pulmonology Group

Cardiometabolic Diseases Group

Cardiometabolic Diseases Group

Cardiometabolic Diseases Group

Cardiometabolic Diseases Group

Cardiometabolic Diseases Group

Cardiometabolic Diseases Group

Cardiometabolic Diseases Group

Cardiometabolic Diseases Group

Cardiometabolic Diseases Group

Cardiometabolic Diseases Group 

Astra Zeneca, Cambridge, United Kingdom

beriamin challis@astrazeneca.com

Cinical Groups

Clinical Groups

Clinical Groups

Genentech, San Francisco, CA, United States

dirk.paul@astrazeneca.com

Julie Hunkapiller

Rion Pendergrass

Audrey Chu

Kirsi Auro
Dermot Reilly

Mermot Reilly

Jaakko Parkkinen

Jaakko Parkkin
Melissa Miller

Melissa Miller

Heikki Joensuu

Olli Carpén

Johanna Mattson
Evelina Salminen

Annika Auranen

Peeter Karihtal
Päiv Auvinen

Klaus Elenius

Johanna Schleutker

Esa Pitkänen

Nina Mars
Mark Daly

Relja Popovic

Befrey Waring

Bridget Riley-Gillis
Anne Lehtonen

Jennifer Schutzman
Julie Hunkapiller penders2@gene.com

Francisco, CA, United States

kirsi.m.auro@gsk.com

dreil111@its.jnj.com

Novartis, Boston, MA, United States

Pfizer, New York, NY, United States

Hospital District of Helsinki and Uusimaa, Helsinki, Finland

Hospital District of Helsinki and Uusimaa, Helsinki, Finland

Hospital District of Helsinki and Uusimaa, Helsinki, Finland

Hospital District of Helsinki and Uusimaa, Helsinki, Finland

Hospital District of Helsinki and Uusimaa, Helsinki, Finland

Pirkanmaa Hospital District, Tampere, Finland

Northern Savo Hospital District, Kuopio, Finland

Hospital District of Southwest Finland, Turku, Fin

mike.mendelson@novartis.com

melissa.r.miller@pfizer.com

tuomo.meretoja@hus.fi

heikki.joensuu@hus.f

olli.carpen@helsinki.fi

johanna.mattson@hus.fi

evelina.e.salminen@hus.fi

anaura@utu.fi

peeter.karihtala@oulu.f

klaus.elenius@utu.fi

Hospital District of Southwest Finland, Turku, Finland

johanna.schleutker@utu. I

Institute for Molecular Medicine Finland (FIMM), HiLlFE, University of Helsinki, Helsinki, Finland esa.pitkanen@helsinki.fi

Institute for Molecular Medicine Finland (FIMM), HiLIFE, University of Helsinki, Helsinki, Finland nina.mars@helsinki.fi
Institute for Molecular Medicine Finland (FIMM), HiLIFE, University of Helsinki, Helsinki, mark.daly@elsinki.fi

Institute for Molecular Medicine Finland (FIMM), HiLIFE, University of Helsinki, Helsinki,

relja.popovic@abbvie.com

Abbie, Chicaso, IL, Uniod States

bridget rileygillis@am

anne.lehtonen@abbvie.com

Abbvie, Chicago, IL, United States

Genentech, San Francisco, CA, United States

schutzman.jennifer@gene.com

hunkapiller.julie@gene.com bowersn1@gene.com

GlaxoSmithKline, Espoo, Finland
Cardiometabolic Disesses Group

Cardiometablis

Cardiometabolic Diseases Group

Cardiometabolic Diseases Group

Cardiometabolic Diseases Group

Cardiometabolic Diseases Group

Cardiometabolic Diseases Group

Cardiometabolic Diseases Group

Cardiotalic Diseases Group

Cardiometabolic Diseases Group

Oncology Group

Oncology Group

Oncology Group

Oncology Group

Oncology Group

Oncology Group

Oncology Group

Oncology Group

Oncology Group

Oncology Group

Oncology Group

Oncology Group

Oncology Group

Oncology Group

Oncology Group

Oncology Group

Oncology Group 
Natalie Bowers

Dre Kula

Diptee Kulk

Kirsi Auro

Alessandro Porello

Andrey Loboda
Heli Lehtonen

Stefan McDonough

Sauli Vuoti

Kai Kaarniranta
Joni A Turunen

Terhi Ollila

Hannu Uusitalo

Esa Pitkänen

Mengzhen L

Heiko Runz

Stephanie Loomis

Erich Strauss

Natalie Bower

Rion Penderar

Rion Pendergrass

Laura Huilaja

Katariina Hannula-Jouppi

Teea Salmi

Sirkku Peltone

Leena Koulu

Nizar Smaoui

Anne Lehtone

David Choy

Rion Pendergrass
Dawn Waterworth
Genentech, San Francisco, CA, United States

Genentech, San Francisco, CA, United Stat

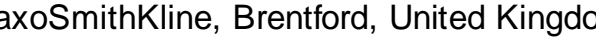

GlaxoSmithKine, Espoo, Finland

nt, LLC, Spring House, PA, United States

erck, Kenilworth, NJ, United States

Pfizer, New York, NY, United States

Janssen-Cilag Oy, Espoo, Finland

Northern Savo Hospital District, Kuopio, Finland

Helsinki University Hospital and University of Hest

Folkhalsan Research Center, Helsinki, Finland
Hospital District of Helsinki and Uusimaa, Helsinki, Finland

inki, Finland

Finland (FIMM), HiLlFE, University of Helsinki, Helsinki, Finland juhna.karjalainen@helsinki.f

Abbvie, Chicago, IL, United States

Biogen, Cambridge, MA, United States

Biogen, Cambridge, MA, United States

Genentech, San Francisco, CA, United States

Genentech, San Francisco, CA, United States

Genentech, San Francisco, CA, United States

Northern Ostrobothnia Hospital District, Oulu, Finland

Northern Ostrobothnia Hospital District, Oulu, Finland

Hospital District of Helsinki and Uusimaa, Helsinki, Finland

Pirkanmaa Hospital District, Tampere, Finland

Hospital District of Southwest Finland, Turku, Finland

Sospital District of Southwest Finland, Turku, Finlan

Abbvie, Chicago, Ih United States

Abbvie, Chicago, II, United States

Genentech, San Francisco, CA, United States

Genentech, San Francisco, CA, United States

Janssen Research \& Development, LLC, Spring House, PA, United States bowersn1@gene.com

penders@gene.com

kirsi.m.auro@gsk.com

APorrell@ITS.JNJ.com

heli.lehtonen@pizer.com

stefan.McDonough@pfizer.con

swoot@@its.jnj.com

jni.turunen@elsinki.fi

terhi.ollila@hus.fi

mengzhen.liu@abbvie.com

heiko.runz@biogen.com

stephanie.loomis@biogen.com

strauss.erich@gene.com

bowersn1@gene.com

penders@gene.com

kaisa.tasanen-maatta@oulu

laura.huilaja@oulu.fi

katariina.hannula-jouppi@hus.

teea.salmi@pshp.

sipelto@utu.fi

leena.koulu@tyks.fi

nizar.smaoui@abbvie.com

anne.ehtonen@abue.com

choy.david@gene.com

penders2@gene.com

dwaterwo@its.jnj.com
Clinical Groups

Clinical Groups

Clinical Groups

Clinical Groups

clinical Groups

Clinical Groups

Clinical Groups

Clinical Groups

Clinical Groups

Clinical Groups

Clinical Groups

Clinical Groups

Clinical Groups

Clinical Groups

Clinical Groups

Clinical Groups

Clinical Groups

Clinical Groups

Clinical Groups

Clinical Groups

Clinical Groups

Clinical Groups

Clinical Groups

Clinical Groups

Clinical Groups

Clinical Groups

Clinical Groups

Clinical Groups
Oncology Group

Oncology Group

Oncology Group

Oncology Group

Oncology

Oncology Group

Opthalmo

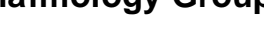

Opthalmology Group

Opthalmology Group

Opthalmology Group

Opthalmology Group

Opthalmology Group

Opthalmology Group

Opthalmology Group

Opthalmology Group

Ophalmology Group

Dermatology Group

Dermatology Group

Dermatology Group

Dermatology Group

Dermatology Group

Dermatology Group

Dermatology Group

Dermatology Group

Dermatology Group

Dermatology Group

Dermatology Group 
Maria Siponen
Lisa Suominen

Päivi Mäntylä

Ulvi Gursoy
Vuokko Anttone

Vuokko Antton
Kirsi Sipilä

Rion Pendergrass

Hannele Lainuori

Venla Kurra

Laura Kotaniemi-Talone

Oskari Heikinheimo

IIkka Kalliala

Lauri Aaltonen

Johannes Kettune
Marja Väaräsmäki

Outi Uimari

Laure Morin-Papunen

Maarit Ninimäki

Terhi Piltonen

Katja Kivinen

Taru Tukiainen

Mary Pat Reeve

Mark Daly
Pfizer, New York, NY, United States

Hospital District of Helsinki and Uusimaa, Helsinki, Finland

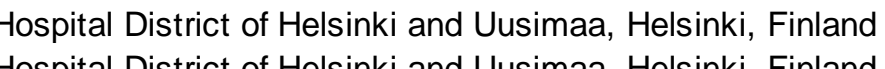

Hospital District of Helsinki and Uusima Helsinki, Finnand

Hospital District of Helsinki and Uusimaa Helsinki, Finland

Hospital District of Helsinki and Uusimaa, Helsinki, Finland

Northerm Savo Hospital District, Kuopio, Finland

Northern Savo Hospital District, Kuopio, Finlan

Northern Savo Hospital Distict, Kuopio, Finland

Hospital District of Southwest Finland, Turku, Finland

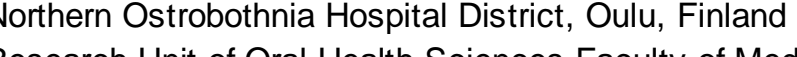

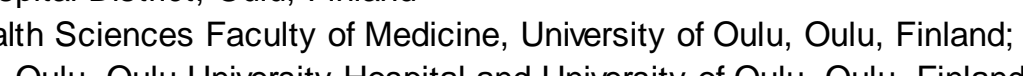

and University of Oulu, Oulu, Finlan

Genentech, San Francisco, CA, United States (Pstiute for Molecular Medicine Finland (FIMM)

Pirkanmaa Hospital Districtict, Tampere, Finland Finland

Hospital District of Helsinki and Uusimaa, Helsinki, Finland

Hospital District of Helsinki and Uusimaa, Helsinki, Finland

Hospital District of Southwest Finsand, Turku, Fintand

Northern Ostrobothnia Hospital District, Oulu, Finland

Northern Ostrobothnia Hospital District, Oulu, Finland

Northern Ostrobothnia Hospital District, Oulu, Finland

Northern Ostrobothnia Hospital District, Oulu, Finland

Northern Ostrobothnia Hospital District, Oulu, Finland

terhi.piltonen@oulu.fi
Northern Ostrobothnia Hospital District, Oulu, Finland
Institute for Molecular Medicine Finland (FIMM), HiLlFE, University of Helsinki, Helsinki, Finland katja.kivinen@helsinki.fi

Institute for Molecular Medicine Finland (FIMM), HiLlFE, University of Helsinki, Helsinki, Finland taru.tukiainen@nelsinki.fi

Institute for Molecular Medicine Finland (FIMM), HiLIFE, University of Helsinki, Helsinki, Finland mary.reeve@helsinki.fi

Institute for Molecular Medicine Finland (FIMM), HiLIFE, University of Helsinki, Helsinki,
Finland; Broad Institute of MIT and Havard; Massachusetts General Hospital

mark.daly@helsinki.fi
Clinical Groups

Cinical Groups

Clinical Groups

Clinical Groups

Clinical Groups

Clinical Groups

Clinical Groups

Clinical Groups

Clinical Groups

Clinical Groups

Clinical Groups

Clinical Groups
Clinical Groups

Clinical Groups

Clinical Groups

Clinical Groups

Clinical Groups

Clinical Groups

Clinical Groups

Clinical Groups

Clinical Groups

Clinical Groups

Clinical Groups

Clinical Groups

Clinical Groups

Clinical Groups

Clinical Groups

Clinical Groups

Clinical Groups

Clinical Groups
Dermatology Group

Dermatology Group

Odontology Group

Odontology Group

Odontology Group

Odontology Group

Odontology Group

Odontology Group

Odontology Group

Odontology Group

Odontology Group

Odontology Group

Odontology Group

Women's Health and Reproduction Group

Women's Health and Reproduction Group

Women's Health and Reproduction Group

Women's Health and Reproduction Group

Women's Health and Reproduction Group

Women's Health and Reproduction Group

Women's Health and Reproduction Group Women's Health and Reproduction Group Women's Health and Reproduction Group Women's Health and Reproduction Group Women's Health and Reproduction Group Women's Health and Reproduction Group Women's Health and Reproduction Group Women's Health and Reproduction Group Women's Health and Reproduction Group Women's Health and Reproduction Group 
Niko Välimäki

Jaakko Tyrmi

Heidi Silven

Reva Sliz

Susanna Savikos

Triin Laisk

Natalia Pujol

Mengzhen Liu

Bridget Riley-Gillis

Rion Pendergrass
Janet Kumar

Janet Kumar

Kirsi Auro

liris Hovatta

Chia-Yen Chen

Kumar Veerapen

Hanna Ollila

Jaana Suvisaari
Thomas Damm Als

Thomas Damm
Antti Mäkitie

University of Helsinki, Helsinki, Finland

University of Oulu, Oulu, Finland / University of Tampere, Tampere, Finland

University of Oulu, Oulu, Finland

University of Oulu, Oulu, Finland

University of Oulu, Oulu, Finland

Estonian biobank, Tartu, Estonia

Estonian biobank, Tartu, Estonia

Abbvie, Chicago, IL, United States

Abbvie, Chicago, IL, United States

, San Francisco, CA, United States

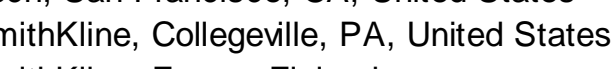

University of Helsinki, Finland

Biogen, Cambridge, MA, United States

Hospital District of Helsinki and Uusimaa, Helsinki, Finland

Broad Institute, Cambridge, MA, United States
Institute for Molecular Medicine Finland (FIMM), HiLIFE, University of Helsinki, Helsinki, Finlan

Finnish Institute for Health and Welfare (THL), Helsinki, Finland

Aarhus University, Denmark

作

Helsinki University Hospital, Helsinki, Finland

Argyro Bizaki-Vallaskangas Pirkanmaa Hospital District, Tampere, Finland

Sanna Toppila-S

Elmo Saarenta

Antti Aarnisalo

Evelina Salminen

Elisa Rahikkala

Johannes Kettune

Helsinki, Finland

$$
\text { Tampere, Finland }
$$

Hospital District of Southwest Finland, Turku, Finland

Hospital District of Helsinki and Uusimaa, Helsinki, Finland

Hospital District of Helsinki and Uusimaa, Helsinki, Finland

Northern Ostrobothnia Hospital District, Oulu, Finland

Transplantation and Liver Surgeny Clinic, Helsinki Univentral Hospital, Helsinki, Finland

Helsinki, Finland niko.valimaki@helsinki.fi Clinical Groups

eija.k.laakkonen@jyu.

jaakko.tyrmi@oulu.fi

eeva.sliz@oulu.fi

riikka.artman@oulu.fi

triin.laisk@ut.ee

natalia.pujolgualdo@oulu.fi

mengzhen.liu@abbvie.com

bridget.rileygillis@abbvie.com

penders2@gene.com

kirsi.m.auro@gsk.com

iiris. hovatta@helsinki.fi

chiayen.chen@biogen.co

hanna.m.ollila@helsinki.f

jaana.suvsaar@.t.fi

antti.makitie@helsinki.fi

argyro.bizaki-vallaskangas@tuni.fi

tytti.willberg@tyks.fi

anti.aarnisalo@hus.fi

elsa.ranikkala@pshp.lif

kristina.aittomaki@helsinki.

fredrik.aberg@helsinki.fi
Clinical Groups

Clinical Groups

clinical Groups

Clinical Groups

Clinical Groups

Clinical Groups

Clinical Groups

Clinical Groups

Clinical Groups

Clinical Groups

Clinical Groups

Clinical Groups

Clinical Groups

Clinical Groups

Clinical Groups

Clinical Groups Clinical Groups

Clinical Groups

Clinical Groups

Clinical Groups

Clinical Groups

Clinical Groups

Clinical Groups

Clinical Groups

Clinical Groups

Clinical Groups
Women's Health and Reproduction Group

Women's Health and Reproduction Group

Women's Health and Reproduction Group

Women's Health and Reproduction Group

Women's Health and Reproduction Group

Women's Health and Reproduction Group

Women's Health and Reproduction Group

Women's Health and Reproduction Group

Women's Health and Reproduction Group

Women's Health and Reproduction Group

Women's Health and Reproduction Group

Won's Healh and Reproduction Group

Depression group

Depression group

Depression group

Depression group

Depression group

Depression group

ENT (ear, nose and throath) Group

ENT (ear, nose and throath) Group

ENT (ear, nose and throath) Group

ENT (ear, nose and throath) Group

ENT (ear, nose and throath) Group

ENT (ear, nose and throath) Grou

ENT (ear, hose and throath) Group

POI (premature ovarian failure) Group

LiverScore Group 
Samuli Ripatti
Mark Daly

Juha Karjalainen

Aki Haullinna

Priit Palta

Shabbeer Hassan

Pietro Della

Wei Zhou

Mutaamba Maash

Kumar Veerapen
Shabbeer Hassan

Susanna Lemmelà

Manuel Rivas

Aarno Palotie

Aoxing Liu

Arto Lehisto

Andrea Ganna
Vincent Llorens

Hannele Laivori

Taru Tukiainen

Henrike Heyne

Nina Mars
Joel Rämö

Joel Ramo
Elmo Saarentaus
Hanna Ollila

Rodos Rodostheno

Rodos Rodosthen
Satu Strausz

Tuula Palotie

Kimmo Palin

samuli.ripatti@nelsinki.fi

Institute for Molecular Medicine, Finland (FIMM), HiLIFE, University of Helsinki, Helsinki,

mark.daly@helsinki.fi

inland; Broad Institute of MIT and Harvard; Massachusetts General Hospita

Institute for Molecular Medicine Finland (FIMM), HiLLFE, University of Helsinki, Helsinki, Finland aki.

Institute for Molecular Medicine Finland (FIMM), HiLIFE, University of Helsinki, Helsinki, Finland juha.mehtonen@helsinki fif Institute for Molecular Medicine Finland (FIMM), HiLLFE, University of Helsinki, Helsinki, Finland priit.palta@helsinki.fi

Institute for Molecular Medicine Finland (FIMM), HiLIFE, University of Helsinki, Helsinki, Finland shabbeer.hassan@helsinki.fi

Institute for Molecular Medicine Finland (FIMM), HiLLFE, University of Helsinki, Helsinki, Finland pietro.dellabriottaparolo@helsinki. Broad Institute, Cambridge, MA, United States

Broad Institute, Cambridge, MA, Unied States

Institute for Molecular Medicine Finland (FIMM), HiLIFE, University of Helsinki, Helsinki, Finland shabbeer.hassan@helsinki.fi

University of Stanford, Stanford, CA, United States
Institute for Molecular Medicine Finland (FIMM), HiLIFE, University of Helsinki, Helsinki, Finland mari.e.niemi@ordelelsinki.fi
Institute for Molecular Medicine Finland (FIMM), HiLIFE, University of Helsinki, Helsinki, Finland aarno.palotie@enelsinki.fii mrivas@stanford.edu Institute for Molecular Medicine Finland (FIMM), HiLIFE, University of Helsinki, Helsinki, Finland aarno.palotie@helsinki. Institute for Molecular Medicine Finland (FIMM), HILIFE, University of Helshnk, Helsinkl, Finland aoxing.liu@helsinki.fi hstue Institute for Molecular Medicine Finnand (FIMM), HiLIFE, University of Helsinki, Helsinki, Finland vincent Illoreans@helsinki fi Institute for Molecular Medicine Finland (FIMM), HiLIFE, University of Helsinki, Helsinki, Finland hannele laiwori@helsinki fi Institute for Molecular Medicine Finland (FIMM), HIILIFE, University of Helsinki, Helsinki, Finland taru tukiainen@helsinki. fr Institute for Molecular Medicine Finland (FIMM), HiLIFE, University of Helsinki, Helsinki, Finland mary.reeve@helsinki.fi Institute for Molecular Medicine Finland (FIMM), HILIFE, University of Helsinki, Helsinki, Finland hheyne@broadinstitute.org Institute for Molecular Medicine Finland (FIMM), HiLIFE, University of Helsinki, Helsinki, Finland nina.mars@helsinki.f Institute for Molecular Meditue Finland (FMM), HLIF, Unversty or Helsikl, Helsinki, Filland joel.ramo@helsinki.fi Institute for Molecular Medicine Finland (FIMM), HiLIFE, University of Helsinki, Helsinki, Finland hanna.m. ollila@helsinki fif Institute for Molecular Medicine Finland (FIMM). HiLIFE, University of Helsinki, Helsinki, Finland rodos rodosthenous@ $@$ helsink fif Institute for Molecular Medicine Finland (FIMM), HiLLFE, University of Helsinki, Helsinki, Finland satu.strausz@helsinki.fi University of Helsinki and Hospital District of Helsinki and Uusimaa, Helsinki, Finland tuula.palotie@nelsinki.fi University of Helsinki, Helsinki, Finland
FinnGen Analysis working gro FinnGen Analysis working group FnnGen Analysis working gro FinnGen Analysis working group FinnGen Analysis working gro FinnGen Analysis working group FinnGen Analysis working grol FinnGen Analysis working group Analysis working group FinnGen Analysis working groo FinnGen Analysis working group FinnGen Analysis working grol FinnGen Analysis working group FinnGen Analysis working grot FinnGen Analysis working group FinnGen Analysis working groo FinnGen Analysis working group FinnGen Analysis working grol FinnGen Analysis working group FinnGen Analysis working groc FinnGen Analysis working group FinnGen Analysis working grol FinnGen Analysis working group FinnGen Analysis working grol FinnGen Analysis working group FinnGen Analysis working grot FinnGen Analysis working group FinnGen Analysis working grol FinnGen Analysis working group FinnGen Analysis working groo FinnGen Analysis working group FinnGen Analysis working gros FinnGen Analysis working group FinnGen Analysis working gro FinnGen Analysis working group FinnGen Analysis working grol FinnGen Analysis working group FinnGen Analysis working grol FinnGen Analysis working group FinnGen Analysis working grot FinnGen Analysis working group FinnGen Analysis working grol FinnGen Analysis working group FinnGen Analysis working grol FinnGen Analysis working group FinnGen Analysis working gro FinnGen Analysis working group FinnGen Analysis working groL FinnGen Analysis working group FinnGen Analysis working gro FinnGen Analysis working group FinnGen Analysis working grot FinnGen Analysis working group FinnGen Analysis working grot FinnGen Analysis working group 
Kristin Tsuo , HiLIFE, University of Helsinki, Helsinki, Fi

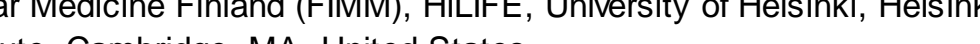

Katri Pylkäs

Eeva Sliz

Minna Karjalainen
Tuomo Mantere

Eeva Kangasniemi

Sami Heikkinen
Arto Mannermaa

Eija Laakkonen

Nina Pitkănen

Samuel Lessard
Clément Chatelain

Perttu Terho

Sirpa Soini

Jukka Partanen
University of Oulu, Oulu, Finland

katri.pylkas@oulu.
eeva.sliz@oulu.fi

Univessty of Oulu, Oulu, Finland

Northern Finland Biobank Finland minna.k.karjalainen@oulu.

Oulu Finand

tuomo.mantere@oulu.fi

Finnish Clinical

eeva.kangasniemi@pshp.fi

sami.heikkinen@uef.fi

Biobank of Eastern Finland / University of Eastern Finland / Northern Savo Hospital District, Kuopio, Finland Translational Sciences, Sanofi R\&D, Framingham, MA, USA Sciences, Sanofi R\&D, Framingham, MA, USA

to.mannermaa@uef.fi

Nina.Pitkanen@tyks.fi

samenticicom

Southwest Finland, Turku, Finland

atelain@sanofi.con
University of Jyväskylä, Jyväskylä, Finland

Auria Biobank / University of Turku / Hospital District of Southwest Finland, Turku, Finland
FinnGen Analysis working grot FinnGen Analysis working group FinnGen Analysis working groo FinnGen Analysis working group FinnGen Analysis working groı FinnGen Analysis working group FinnGen Analysis working grot FinnGen Analysis working group FinnGen Analysis working grol FinnGen Analysis working group
FinnGen Analysis working gro FinnGen Analysis working group FinnGen Analysis working grot FinnGen Analysis working group Finn FinnGen Analysis working grol FinnGen Analysis working group

FinnGen Analysis working grot FinnGen Analysis working group FinnGen Analysisworking grot FinnGen Analysis working group FinnGen Analysis working grot FinnGen Analysis working group Fingen Analysis workng grot FinnGen Analysisworking group FinnGen Analysis working groL FinnGen Analysis working group FinnGen Analysis working gro FinnGen Analysis working group
FinnGen Analysis working grot FinnGen Analysis working group FinnGen Analysis working grot FinnGen Analysis working group innGen Analysis working grot FinnGen Analysis working group Fingen Biobank directors Biobank directors Biobank directors Biobank directors Biobank directors 

Institute for Molecular Medicine Finland (FIMM), HiLIFE, University of Helsinki, Helsinki, Finland huei-yi.shen@helsinki.fi

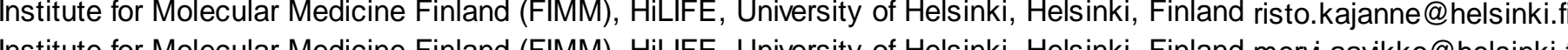

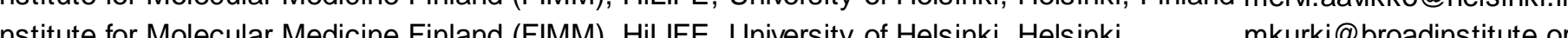
Juha Karjalainen Arto Lehisto Juha Mehtonen Wei Zhou Masahiro Kanai Mutaamba Maash Kumar Veerapen Aki Haullinna Susanna Lemmelä Tuomo Kiiskinen L. Elisa Lahtela Mari Kaunisto Elina Kilpelainen Timo P. Sipilä Institute for Molecular Medicine Finland (FIMM), HiLLFE, University of Helsinki, Helsinki, Finland pietro.dellabriottaparolo@helsinki. Institute for Molecular Medicine Finland (FIMM), HiLLFE, University of Helsinki, Helsinki, Finland arto.lehisto@helsinki.fi Institute for Molecular Medicine Finland (FIMM), HiLLFE, University of Helsinki, Helsinki, Finland juha.mehtonen@helsinki.fi Broad Institute, Cambridge, MA, United States wzhou@broadinstitute.org Broad Institute, Cambridge, MA, United States mkanai@broadinstitute.org Broad Instive, Cambide, MA, United States mmanha@broadinstitute.org

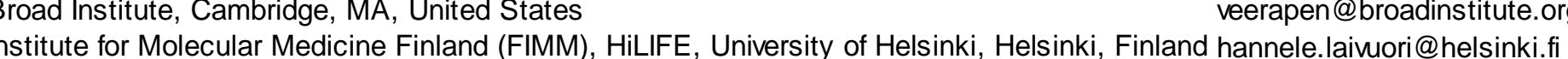
Institute for Molecular Medicine Finland (FIMM). HiLIFE, University of Helsinki, Helsinki, Finland aki.havlinna@helsinki.fi Institute for Molecular Medicine Finland (FMMM), HiLIFE, University of Helsinki, Helsinki, Finland susanna.lemmela@helsinki.f Institute for Molecular Medicine Finland (FIMM), HiLLFE, University of Helsinki, Helsinki, Finland tuomo.kiiskinen@helsinki.fi Institute for Molecular Medicine Finland (FMMM), HiLIFE, University of Helsinki, Helsinki, Finland laura.lahtela@helsinki.fi

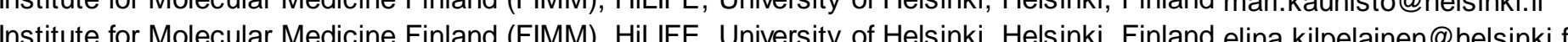

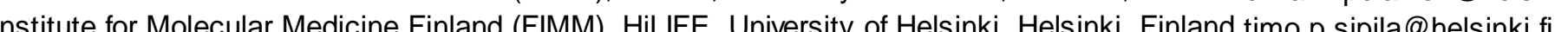

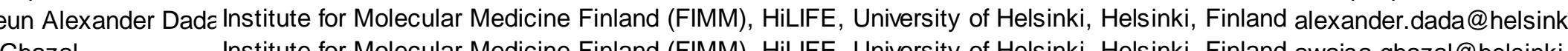
Awaisa Ghazal Institute for Molecular Medicine Finland (FIMM), HiLIFE, University of Helsinki, Helsinki, Finland awaisa.ghazal@helsinki.fi Anastasia Kytölä
Rigbe Weldatsadik

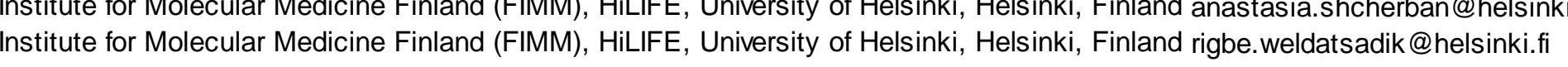

FinnGen Teams FinnGen Teams Finn Teams FinnGen Teams
FinnGen Teams

\section{Administration}

Administration

Administration
Administration Administration

FinnGen Teams FnnGen Teams FinnGen Teams FinnGen Teams FinnGen Teams FinnGen Teams FinnGen Teams FinnGen Teams FinnGen Teams FinnGen Teams FinnGen Teams FinnGen Teams FinnGen Teams

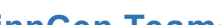
FinnGen Teams FinnGen Teams FinnGen Teams

Analysis 
Kati Donner

Anu Loukola

Päiv Laiho

Tuuli Sistonen

Markku Laukkane

Elina Järvensivu

Sini Lahteenmmä

Regis Wong
Auli Toivola

Minna Brunfeldt

Kati Kristiansson

Kati Kristiansson
Susanna Lemmelà

Sami Koskelainen
Tero Hiekkalinna

Teemu Paajanen

Priit Palta

Kalle Pärn

Mart Kals

Vishal Sinha

Taria Laitinen

Mary Pat Reeve

Marianna Niemi

Kumar Veerape

Harri Siirtola

Javier Gracia-Tabue
Mika Helminen

Mika Helminen
Tina Luukkaala

lida Vähätalo

Jyrki Pitkänen
Marco Hautalah
Institute for Molecular Medicine Finland (FIMM), HiLIFE, University of Helsinki, Helsinki, Finland kati.donner@helsinki.fi sinki, Helsinki, Finland timo.p.sipila@helsinki.fi Helsinki Biobank / Helsinki University and Hospital District of Helsinki and Uusimaa, Helsinki anu.loukola@hus. THL Biobank / Finnish Insthue for Health and Wellare (THL), Helsinki, Finland

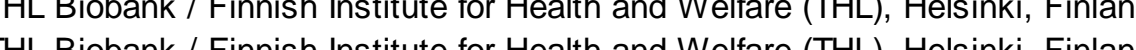
THL Biobank / Finnish Institute for Health and Welfare (THL). Helsinki, Finland THL Biobank / Finnish Institute for Health and Welfare (THL), Helsinki, Finland THL Biobank / Finnish Institute for Health and Welfare (THL), Helsinki, Finland THL Biobank / Finnish Institute for Health and Welfare (THL), Helsinki, Finland THL Biobank / Finnish Institute for Health and Welfare (THL), Helsinki, Finland THL Biobank / Fnish Instute tor Heall hand Wellare (THL), Helsinki, Finlan THL Bibank / Finsish Instite for Heallh and Weltere (THL), Helsinki, Finland THL Biobank / Finnish Institute for Health and Welfare (THL). Helsinki. Finland Institute for Molecular Medicine Finland (FIMM), HiLLFE, University of Helsinki, pail.laho@m.th tuuli.sistonen@thl.fi markku.laukkanen@th.f elina.jarvensivu@thl.fi sini.lahteenmaki@thl. lotta.mannikko@thl.fi regis.wong@thl.fi
auli.toivola@thl.fi auli.toivola@thl.fi minna.bruntelden.if kati.kristiansson@thl.fi

THL Biobank / Finnish Institute for Health and Welfare (THL), Helsinki, Finland

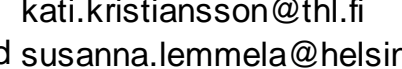
THL Biobank / Finnish Institute for Health and Welfare (THL), Helsinki, Finland
THL Biobank / Finnish Institute for Health and Welfare (THL). Helsinki, Finland THL Biobank / Finnish Institute for Health and Welfare (THL), Helsinki, Finland sami.koskelainen@thl.fi Institute for Mocur Institute for Molecular Medicine Finland (FIMM), HiLIFE, University of Helsinki, Helsinki, Finland shuang luo Institute for Molecular Medicine Finland (FIMM), HiLLFE, University of Helsinki, Helsinki, Finland vishal sinna@@elsinkifi Pirkanmaa Hospital District, Tampere, Finland
Institute for Molecular Medicine Finland (FIMM), HiLlFE, University of Helsinki, Helsinki, Finland (a) University of Tampere, Tampere, Finland Broad Institute, Cambridge, MA, United States

University of Tampere, Tampere, Finland

University of Tampere, Tampere, Finland

University of Tampere, Tampere, Finland

University of Tampere, Tampere, Finlan
Institute for Molecular Medicine Finland

Finnish Biobank Cooperative - FINBB
FinnGen Teams

FinnGen Teams

FinnGen Teams FinnGen Teams FinnGen Teams FinnGen Teams FinnGen Teams FinnGen Teams FinnGen Teams FinnGen Teams FinnGen Teams FinnGen Teams FinnGen Teams FinnGen Teams FinnGen Teams FinnGen Teams FinnGen Teams FinnGen Teams FinnGen Teams FinnGen Teams FinnGen Teams FinnGen Teams FinnGen Teams FinnGen Teams FinnGen Teams FinnGen Teams FinnGen Teams FinnGen Teams FinnGen Teams FinnGen Teams FinnGen Teams
Genotyping

Genotyping

Sample Collection Coordination Sample Logistics

Sample Logistics

Sample Logistics

Sample Logistics

Sample Logistics

Sample Logistics

Sample Logistics

Sample Logistics

Registry Data Operation

Registry Data Operations

Registry Data Operations

Registry Data Operations

Registry Data Operations

Registry Data Operations

Sequencing Informatics

Sequencing Informatics

Sequencing Informatics
Sequencing Informatics

Sequencing Informatics

Trajectory

Trajectory

Trajectory

Trajectory

Trajectory

Trajectory

Trajectory

Trajectory
Trajectory

Data protection officer

FINBB - Finnish biobank cooperative 
Johanna Mäkelä

Sarah Smith

Finnish Biobank Cooperative - FINBB

Finnish Biobank Cooperative - FINBB johanna.makela@finbb.

sarah.smith@finbb.fi
FinnGen Teams

FinnGen Teams

FinnGen Teams

FNBB - Finnish biobank cooperative

FINBB - Finnish biobank cooperative

FINBB - Finnish biobank cooperative 